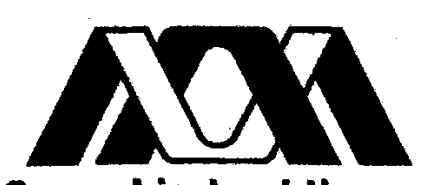

cansaberas at themo

UNIVERSIDAD AUTÓNOMA METROPOLITANA

Unidad Iztapalapa

\author{
DIVISIÓN DE CIENCIAS BÁSICAS E INGENIERÍA
}

\title{
Inconexión acíclica de torneos
}

\author{
Tesis que presenta \\ José Luis Cosme Álvarez \\ Para obtener el grado de \\ Doctor en Ciencias (Matemáticas)
}

Asesor: Dr. Bernardo Llano Pérez

Jurado calificador:

Presidente: Dr. Eduaydo Rivera Campo

Secretario: Dra. Mika Olsen alficher

Vocales: Dra. Hortensia Galeana Sánchez

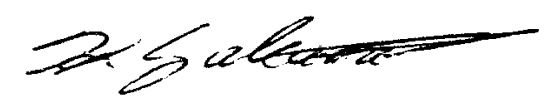

Dra. Rita Esther Zuazua Vega nito stets

Dr. Bernardo Llano Pérez

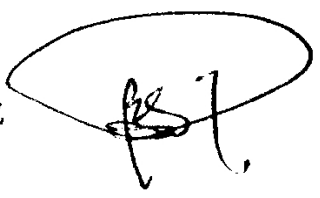

México D. F., 2 de diciembre de 2014 
José Luis Cosme Álvarez Posgrado en Matemáticas

Universidad Autónoma Metropolitana

\section{Inconexión acíclica} de torneos 



\section{Agradecimientos}

C

onsidero que a las primeras personas que debo agradecer es a mi familia. A mi madre, la Sra. Rita Álvarez Santiago y a mis hermanos: Alejandro, Irene, Filiberto y Gustavo por demostrarme que si en alguien puedo confiar, será siempre en la familia. Un agradecimiento especial a mi hermano Gustavo por ser mi compañero y amigo durante estos últimos años, en los que hemos compartido momentos buenos y malos. En todo este tiempo nos hemos apoyado mutuamente y he visto tu desarrollo personal y profesional, y eso hermano, es lo mejor que la vida nos puede brindar.

A la Universidad Autónoma Metropolitana, Unidad Iztapalapa, de la que me he sentido parte desde mi primer día en ella.

Al Departamento de Matemáticas y a sus profesores de los cuales me llevo gratos recuerdos y algunos no tanto, pero que al final son parte del aprendizaje.

Tengo que hacer un reconocimiento a los Doctores Mario Pineda Ruelas y Joaquín Delgado Fernández, quienes estuvieron al frente del Departamento durante mi paso por el posgrado. Les agradezco que hayan confiado en mí, al invitarme y apoyarme para trabajar en este departamento.

A mi asesor y mentor, el Doctor Bernardo Llano de quien he aprendido casi todo lo que sé de la Teoría de Gráficas. Si bien es cierto que es una persona firme en el aspecto académico, sin embargo me ha mostrado su lado amable y la excelente persona que hay detrás. Le agradezco el apoyo incondicional en todos los sentidos, sus enseñanzas, su guía, su paciencia y sobre todo su amistad durante estos últimos años. A través de su mano firme -pero siempre amable-, puede ver mis capacidades y limitaciones, y es por ello que admiro su desinteresada vocación de inculcar y alentar el crecimiento como matemático a toda persona que acude a usted.

A las Doctoras Hortensia Galeana Sánchez y Mika Olsen, así como al Doctor Eduardo Rivera Campo, por haberse tomado el tiempo para leer este trabajo, además de los comentarios y observaciones que hicieron para la mejora del mismo. Les reitero mi profunda admiración.

Quiero agradecer especialmente a la Doctora Rita Esther Zuazua Vega, profesora de la Facultad de Ciencias de la UNAM. Si bien le agradezco el haber revisado este trabajo, pero también tengo muchas otras cosas que agradecerle, pues a través de su desinteresada guía, he conocido otros aspectos de la Teoría de Gráficas. Espero que esa pasión por despertar la inquietud matemática en los alumnos jóvenes, siga por mucho tiempo. Reconozco y admiro su especial empeño en la colaboración y apoyo a los alumnos realizado talleres, seminarios y demás eventos. Me considero afortunado por haber sido parte de muchos de estos encuentros, pero sobre todo, de haberla conocido.

$\mathrm{Al}$ posgrado en Matemáticas y a los compañeros del mismo. Durante mi estancia al frente de la sala del Posgrado en Matemáticas y del Seminario del Posgrado, conocí a muchas personas y también he visto llegar e irse a otras tanta en su paso por el posgrado, 
pero todas ellas han dejado algo. Quiero agradecer especialmente a dos personas que, aparte de ser compañeros y ahora colegas, llegamos a ser grandes amigos, a Rocío Meza Moreno y a Alejandro Sánchez Peralta. Gracias a los dos por hacer de esta etapa algo memorable.

Al Consejo Nacional de Ciencia y Tecnología (CONACyT) por el apoyo económico.

Hay personas a las que he conocido, algunas se han ido y otras siguen aquí, pero todas ellas han sido parte de esta etapa, por lo que les agradezco ser parte de esto.

Finalmente, tengo que reconocer a una persona que, a pesar de que nunca conocí en persona, es la razón del tema de esta tesis: a Víctor Neumman-Lara por el legado que ha dejado y del que ahora soy parte. Esta tesis es una clara muestra de que él sigue presente en la forma de problemas, teoremas, corolarios, entre muchas otras cosas.

A todos ustedes muchas gracias.

J. L. Cosme Álvarez 


\section{Índice general}

Agradecimientos $\quad$ I

Introducción $\quad$ V

1. Preliminares 1

1.1. Terminología y conceptos básicos . . . . . . . . . . . . . . . 1

1.2. Inconexión acíclica e inconexión libre de $\vec{C}_{3} \ldots \ldots$. . . . . . . . 4

1.3. Torneos . . . . . . . . . . . . . . . . . . . 7

1.4. Sumas de Zykov . . . . . . . . . . . . . . . . . . . 10

1.5. Generalización del concepto de inconexión acíclica y libre de $\vec{C}_{3} \ldots$. . 15

2. La diferencia $\overrightarrow{\omega_{3}}(T)-\vec{\omega}(T) \quad 23$

2.1. El epimorfismo $\pi$ y la digráfica auxiliar $D_{n+1} \ldots \ldots$. . . . . . . . . . 23

2.2. El torneo $T_{9}$ y la familia $\mathfrak{V}_{n} \ldots \ldots$. . . . . . . . . . . . . 25

2.3. Inconexión acíclica y libre de $\vec{C}_{3}$ de los elementos de la familia $\mathfrak{V}_{n} \ldots . \quad 32$

3. Una conjetura sobre la tensión 37

3.1. Pares discordantes . . . . . . . . . . . . . . . . . . . . 37

3.2. Familias de torneos regulares simples y tensos . . . . . . . . . . . . . . 41

3.3. Una familia de torneos regulares, simples y no tensos . . . . . . . . . . 42

4. Torneos regulares tensos y no tensos $\quad 51$

4.1. Ciclos en torneos multipartitos . . . . . . . . . . . . . . 51

4.2. Trayectorias prohibidas en torneos no tensos . . . . . . . . . . . . . . 53

4.3. Torneos tensos y no tensos . . . . . . . . . . . . . . . . . 64

$\begin{array}{ll}\text { 5. Otros resultados sobre la tensión } & 77\end{array}$

5.1. Gráficas bipartitas y torneos regulares tensos . . . . . . . . . . . 77

5.2. Inconexión acíclica de torneos semirregulares . . . . . . . . . . . . . . . 82

5.3. Torneos regulares críticos . . . . . . . . . . . . . . . . . . . 87

5.4. Factorización de torneos y la gráfica $G_{3}(D) \ldots \ldots$. . . . . . . . . 93

$\begin{array}{lr}\text { Conclusiones } & 103\end{array}$

$\begin{array}{ll}\text { Bibliografía } & 105\end{array}$ 


\section{Introducción}

$\mathrm{E}_{\mathrm{n}}$ cas, al famoso Augustus De Morgan en University College London. Frederick le describió a De Morgan el descubrimiento de su hermano mayor Francis. Mientras Francis coloreaba un mapa de Inglaterra, él observó que necesitaba cuatro colores para colorearlo de tal manera que las regiones vecinas tuvieran distinto color y había conjeturado que no más de cuatro colores eran necesarios para colorear dicho mapa. El problema que se planteó Francis Guthrie es el siguiente:

¿Es posible colorear cualquier mapa con solo cuatro colores, de tal forma que dos países vecinos nunca tengan el mismo color?

Este problema -que no fue resuelto sino hasta un siglo después por Kenneth Appel y Wolfgang Haken-, es conocido como el problema de los cuatro colores y es considerado como uno de los detonantes en el surgimiento de la rama de las matemáticas, conocida como Teoría de Gráficas, en particular la coloración de gráficas. Este problema introduce el concepto de número cromático $\chi(G)$, que se define como el menor número de colores necesarios para colorear los vértices de una gráfica $G$, de tal manera que cualquier par de vértices adyacentes no tengan el mismo color.

En 1982 Víctor Neumann-Lara -e independientemente H. Jacob y H. Meyniel en [JM83] - introduce en [NL82] el concepto de número dicromático $d c(D)$ para una digráfica $D$, que es una generalización del número cromático, y se define como el mínimo número de colores necesarios para colorear los vértices de $D$, de tal manera que cada clase cromática sea acíclica. Este trabajo proporciona un nuevo campo de estudio en lo que a teoría de digráficas se refiere y es uno de los pilares de la escuela mexicana de teoría de gráficas que Neumann-Lara fundó.

En 1999 Neumann-Lara introduce en [NL99] otro invariante numérico, que generaliza el número de componentes conexas $\omega(G)$ de una gráfica $G$ : la inconexión acíclica $\vec{\omega}(D)$ para una digráfica $D$, y se define como el máximo número de componentes conexas de la subdigráfica inducida, que se obtiene al borrar un conjunto acíclico de flechas. Esta definición es equivalente a calcular el máximo número de colores para colorear los vértices de $D$, de tal forma que no se formen ciclos dirigidos bien coloreados.

Tanto el número cromático $d c(D)$ como la inconexión acíclica $\vec{\omega}$, miden la complejidad de la estructura cíclica de la digráfica $D$ y derivan propiedades importantes, ya que mientras el número dicromático $d c(D)$ crece proporcionalmente a medida que aumenta la complejidad de su estructura cíclica, en contraste, su inconexión acíclica $\vec{\omega}(D)$ decrece a medida que aumenta.

En [NL99], Neumann-Lara define otro concepto derivado de la inconexión acíclica: la inconexión libre de triángulos o de $\overrightarrow{C_{3}}, \overrightarrow{\omega_{3}}(D)$ para una digráfica $D$, que se restringe a analizar solo los triángulos dirigidos y proporciona una cota superior para la 
inconexión acíclica, es decir $\vec{\omega}(D) \leq \vec{\omega}_{3}(D)$. Si la digráfica $D$ es un torneo $T$, entonces es inmediato ver que toda coloración de sus vértices con dos colores es libre de $\overrightarrow{C_{3}}$ heterocromáticos. Un torneo $T$ tal que $\overrightarrow{\omega_{3}}(T)=2$, lo llamaremos tenso.

Por otra parte, se establece en el mismo artículo [NL99], que $\vec{\omega}_{3}^{+}(D):=\vec{\omega}_{3}(D)+1$ es el mínimo número $r$ tal que toda $r$-coloración de los vértices de $D$, deja al menos un triángulo dirigido heterocromático. Notemos que esta definición es alternativa para la inconexión libre de $\vec{C}_{3}$ y se sigue inmediatamente que un torneo $T$ es tenso si y solo si $\overrightarrow{\omega_{3}}(T)=2$, si y solo si $\vec{\omega}_{3}^{+}(T)=3$.

La concepción de las ideas que dieron origen al concepto de tensión en gráficas, se remontan a los años 70, sin embargo, no fue sino hasta 1992 en el que Jorge Luis Arocha, Javier Bracho y Víctor Neumann-Lara definen la tensión en términos de hipergráficas en [ABNL92].

Recordemos que una hipergráfica $H$ se define por un conjunto de vértices $V$ y un conjunto de hiperaristas $E$, donde $E$ es un conjunto de subconjuntos de $V$. Si consideramos que las aristas de una gráfica son subconjuntos de cardinalidad dos, entonces una hipergráfica es la generalización natural del concepto de gráfica, al tomar subconjuntos de cualquier cardinalidad. Así mismo, recordemos que una gráfica $G$ es conexa si y solo si para toda 2-coloración de sus vértices, existe una arista cuyos vértices son de distinto color, es decir induce una arista heterocromática. Si consideramos a las aristas de una gráfica como un ciclo de longitud dos, entonces toda 2-coloración deja siempre un ciclo bien coloreado y podemos pensar a la inconexión acíclica como una generalización del concepto de conexidad en hipergráficas, es decir, una gráfica es conexa si y solo si es tensa como hipergráfica.

Una 3-hipergráfica uniforme o simplemente una 3-gráfica $H$, es la hipergráfica definida sobre un conjunto de vértices $V(H)$ y un conjunto de hiperaristas que son llamadas 3-aristas, que son el conjunto

$$
E(H)=\{S \subset V(H):|S|=3\} .
$$

En [ABNL92, ABNL95] se define el número heterocromático $h c(H)$ como el mínimo número de colores $r$, de tal manera que toda $r$-coloración de los vértices de $H$, induce al menos una 3-arista heterocromática. Decimos que $H$ es tensa si $h c(H)=3$.

Para un torneo $T, \vec{\omega}_{3}^{+}(T):=\vec{\omega}_{3}(T)+1$ es el número heterocromático $h c\left(H_{T}\right)$ de la 3-gráfica $H_{T}=(V, E)$ que se obtiene de $T$ al definir $V\left(H_{T}\right)=V(T)$ y el conjunto de 3-aristas como

$$
E\left(H_{T}\right)=\left\{S \subseteq V(T): T\langle S\rangle \cong \overrightarrow{C_{3}}\right\},
$$

es decir, el conjunto de 3-aristas serán las ternas de vértices que inducen un triángulo dirigido en $T$. Observemos que $T$ es tenso si y solo si $H_{T}$ es tensa, es decir,

$$
\vec{\omega}_{3}^{+}(T)=h c\left(H_{T}\right)=3 .
$$

En [NL99] Neumann-Lara demuestra que si un torneo regular no es simple, es decir, es isomorfo a una composición, entonces no es tenso y conjetura que el recíproco es también cierto, esto es: 
Un torneo regular es simple si y solo si no es una composición.

La creencia de la veracidad de esta conjetura, fue reforzada con los trabajos posteriores de Hortensia Galeana-Sánchez, Bernardo Llano, Mika Olsen y Víctor Neumann-Lara en [NL99], [LlO07], [NLO09], [GSNL00] y [LINL07], en los que demuestran la tensión de familias infinitas de torneos. Dichos torneos tienen la característica de ser simples y así, los únicos ejemplos de torneos regulares que se saben que no son tensos, son las composiciones, lo que parece confirmar la conjetura de Neumann-Lara.

En este trabajo de investigación, proponemos una familia infinita de torneos regulares, cuyos elementos no son tensos y tampoco son composiciones, lo que demuestra que la conjetura es falsa. No obstante, los miembros de esta familia resultan ser sumas de Zykov, por lo que reformulamos dicha conjetura en un sentido más amplio:

Un torneo es tenso si y solo si no es isomorfo a una suma de Zykov no trivial,

es decir, es primo. Más aún, demostramos que esta reformulación de la conjetura es cierta y damos tres caracterizaciones para los torneos regulares tensos. Estas caracterizaciones, permiten decir más acerca de los torneos regulares tensos, ya que proporcionan por un lado algunos resultados concernientes a los torneos semirregulares y por otro es un auxiliar para determinar una partición del conjunto de vértices del torneo, que realiza la inconexión acíclica y la inconexión libre de $\overrightarrow{C_{3}}$. Finalmente damos algunos resultados que describen la factorización de un torneo como una suma de Zykov no trivial.

Este trabajo se encuentra dividido en cinco capítulos.

El capítulo 1 contiene las definiciones y propiedades básicas sobre la inconexión acíclica e inconexión libre de $\vec{C}_{3}$, así como de los torneos y las sumas de Zykov, que utilizaremos para demostrar algunas propiedades en los capítulos siguientes. Algunos resultados son clásicos en la teoría de torneos y otros tantos son tomados de [NL99], en donde se exponen las generalizaciones de los conceptos de inconexión acíclica e inconexión libre de $\overrightarrow{C_{3}}$. Así mismo, exponemos algunos resultados propios que facilitan las demostraciones en capítulos posteriores.

En el capítulo 2 retomamos el ejemplo 4.2 de [NL99], en el que Neumann-Lara propone un torneo $T_{9}$ no regular de orden 9 y demuestra que $\vec{\omega}\left(T_{9}\right)=2$ y $\overrightarrow{\omega_{3}}\left(T_{9}\right)=3$, lo que prueba que la desigualdad $\vec{\omega}(T) \leq \vec{\omega}_{3}(T)$ puede ser estricta y plantea el siguiente problema (problema 6.6.3 de [NL99]):

¿Existe algún torneo $T$ para el cual $\vec{\omega}(T)=2$ y $\overrightarrow{\omega_{3}}(T)$ es arbitrariamente grande?

En este capítulo damos respuesta afirmativa a este problema, para lo que proponemos, describimos y demostramos las propiedades de la familia $\mathfrak{V}_{n}$, la cual es una generalización del ejemplo propuesto por Neumann-Lara. 
Esta familia proporciona uno de los resultados de mayor interés, pues no solo contesta afirmativamente al problema planteado por Neumann-Lara, sino que generaliza esta idea, pues demostramos que

Para todo par de enteros $r, s$ con $2 \leq r \leq s$, existe un torneo $T$, tal que $\vec{\omega}(T)=r_{\mathrm{y}} \overrightarrow{\omega_{3}}(T)=s$.

En el capítulo 3 hacemos un breve estudio de algunos conceptos sobre pares discordantes en torneos, que son una fuente de inspiración para construir una clase de torneos regulares que no son composiciones y no son tensos. Esta clase de torneos muesta que la conjetura sobre la tensión de torneos regulares simples no es cierta, sin embargo, los elementos de esta clase de torneos pueden ser factorizados como sumas de Zykov no triviales, por lo que proponemos una nueva conjetura sobre la tensión de los torneos regulares primos.

En el capítulo 4 hacemos un breve resumen sobre algunos resultados sobre ciclos en torneos multipartitos, lo que permite demostrar un lema que consideramos fundamental, para demostrar que existen ciertas clases de trayectorias prohibidas en los torneos regulares no tensos. Con lo anterior, finalmente demostramos que la conjetura mencionada anteriormente es cierta, esto es, demostramos que:

Todos los torneos que no son isomorfos a una suma de Zykov son tensos.

En la segunda parte, damos respuesta afirmativa a otros dos problemas planteados por Víctor Neumann-Lara en [NL99] a saber:

- ¿Se cumple que $\overrightarrow{\omega_{3}}(T)=\vec{\omega}(T)$ para todo torneo regular o circulante?

- ¿Será cierto que los torneos regulares son $\overrightarrow{\omega_{3}}$-agudos o $\vec{\omega}$-agudos?

En el capítulo 5 damos una serie de resultados derivados de los capítulos anteriores, acerca de la tensión de torneos regulares. En la primera parte definimos una subdigráfica de un torneo, la cual resulta ser una digráfica bipartita y que permite demostrar una segunda caraterización de los torneos regulares tensos. Más aún, esta subdigráfica proporciona un método para determinar una partición -y por lo tanto, una coloración- del conjunto de vértices de un torneo regular, que realiza la inconexión acíclica y la inconexión libre de $\overrightarrow{C_{3}}$.

En la segunda parte, damos algunos resultados concernientes a la inconexión acíclica y la inconexión libre de $\overrightarrow{C_{3}}$ de torneos semirregulares, esto con la ayuda de la digráfica bipartita antes mencionada.

En la tercera parte, abordamos el problema de determinar si existe alguna clase de torneos regulares que son críticos en vértices con respecto a algún parámetro dado y exhibimos una clase de torneos críticos en vértices con respecto a la inconexión acíclica y la inconexión libre de $\vec{C}_{3}$.

En la cuarta parte proponemos un tipo de gráficas de intersección asociado a un torneo regular. Con la ayuda de esta gráfica, damos una tercera caracterización 
de los torneos regulares tensos, la cual se basa en el estudio de la conexidad de dicha gráfica de intersección. Por otra parte, esta gráfica permite dar un método para factorizar un torneo regular no primo en componentes de una suma de Zykov no trivial.

Finalmente solo resta decir, que este trabajo sigue la esencia heredada de la Escuela Mexicana de Teoría de Gráficas fundada por Víctor Neumann-Lara y está dedicado a estudiantes de ciencias y aquellas personas que reconozcan en esta área, un campo de investigación fértil y productivo. 


\section{Preliminares}

En el presente capítulo proporcionamos un panorama general de los conceptos y herramientas, que nos ayudarán a probar propiedades de los torneos en los capítulos posteriores. La notación, definiciones y teoría en general, fue tomada de [BM76] y [BJG08].

A lo largo del presente capítulo, cuando aparezca un concepto seguido de otro entre paréntesis precedido por «resp.» (respectivamente), daremos por entendido que es posible intercambiarlos para formar una nueva definición, proposición, teorema, etcétera, dependiendo del contexto en el que se encuentre.

\subsection{Terminología y conceptos básicos}

Una gráfica

$$
G=(V(G), E(G))
$$

se define sobre un conjunto de vértices $V(G)$ y aristas $E(G)$. Si no existe confusión de cual es la gráfica a la que nos referimos, entonces simplemente se denotarán por $V$ y $E$ a sus conjuntos de vértices y aristas respectivamente. El orden $|G|$ de una gráfica $G$ es la cardinalidad de su conjunto de vértices $|V(G)|$.

Dos vértices son adyacentes si existe una arista que los une. Dos aristas son incidentes si tienen al menos un vértice en común. Una orientación o dirección es la asignación de un sentido a las aristas, entonces llamadas flechas.

Una digráfica

$$
D=(V(D), A(D))
$$

es una gráfica a la cual se le ha asignado alguna orientación y se define sobre un conjunto de vértices $V(D)$ y flechas $A(D)$. Dados $u, v \in V(D)$, una flecha que va de $u$ a $v$ la denotaremos como $u \rightarrow v$ o $u v \in A(D)$, dependiendo del contexto en el que se encuentre. A una gráfica $G$ la consideraremos en general como una digráfica simétrica, es decir para todo par de vértices $u, v \in V(G)$, se tiene que $u \rightarrow v$ y $v \rightarrow u$ son flechas en $A(D)$ y la denotamos por $u \leftrightarrow v$. La gráfica subyacente de la digráfica $D$ se obtiene al eliminar la orientación de las flechas de la digráfica $D$.

Si $v \rightarrow u$ es una flecha en $D$, diremos que $u$ es ex-vecino de $v$ y en contraste, que $v$ es in-vecino de $u$. A los conjuntos de ex-vecinos e in-vecinos del vértice $v$ los denotaremos por $N^{+}(v, D)$ y $N^{-}(v, D)$ respectivamente. Si no hay confusión sobre cual es la digráfica a la que nos referimos, simplemente denotaremos por $N^{+}(v)$ y $N^{-}(v)$ el conjunto de ex-vecinos e in-vecinos respectivamente. La vecindad $N(v)$ de $v$ es el conjunto de ex-vecinos e in-vecinos, es decir $N(v)=N^{+}(v) \cup N^{-}(v)$. Si $S \subset V(D)$, 
entonces

$$
N^{+}(S)=\left(\bigcup_{v \in S} N^{+}(v)\right) \backslash S \quad \text { y } \quad N^{-}(S)=\left(\bigcup_{v \in S} N^{-}(v)\right) \backslash S .
$$

El ex-grado e in-grado de un vértice $v$ se definen como $\left|N^{+}(v)\right| \mathrm{y}\left|N^{-}(v)\right|$ respectivamente y se denotan por $d^{+}(v, D)$ y $d^{-}(v, D)$ respectivamente o simplemente por $d^{+}(v)$ y $d^{-}(v)$ si se sobreentiende la digráfica $D$.

Diremos que un vértice $v$ es una fuente, si su in-vecindad es vacía y tiene al menos un ex-vecino. Similarmente, diremos que un vértice $v$ es un pozo, si su ex-vecindad es vacía y tiene al menos un in-vecino. La cardinalidad de una fuente y un pozo es el número de ex-grado y in-grado respectivamente.

Una subgráfica $B$ (resp. subdigráfica) es una gráfica (resp. digráfica) contenida en la gráfica $G$ (resp. digráfica $D$ ) y la denotaremos por $B \subseteq G$ (resp. $B \subseteq D$ ). Dadas dos subdigráficas $B_{1}$ y $B_{2}$ de $D$, si $u \rightarrow v$ para toda $u \in B_{1}$ y toda $v \in B_{2}$, entonces lo denotaremos por $B_{1} \Rightarrow B_{2}$.

Diremos que una subdigráfica

$$
P: v_{0} \rightarrow v_{1} \rightarrow \ldots \rightarrow v_{n-1} \rightarrow v_{n}
$$

es una trayectoria de longitud $n$ si la flecha $v_{i-1} \rightarrow v_{i}$ está en $A(D)$, para toda $i \in\{1,2, \ldots, n\}$, con $v_{i} \neq v_{j}$ si $i \neq j$. La denotaremos por $v_{0} \rightsquigarrow v_{n}$ y por simplicidad, diremos que es una $\left(v_{0}, v_{n}\right)$-trayectoria. Un ciclo $\vec{C}_{n}$ de longitud $n$ es una trayectoria cerrada, es decir

$$
\vec{C}_{n}: v_{0} \rightarrow v_{1} \rightarrow \ldots \rightarrow v_{n-1} \rightarrow v_{0}
$$

En particular si $n=3$, diremos que es un triángulo dirigido, simplemente un triángulo o un $\overrightarrow{C_{3}}$. Si $n=4$, diremos que es un cuadrado dirigido, simplemente un cuadrado o un $\vec{C}_{4}$. Decimos que la digráfica $D$ es acíclica si no contiene ciclos como subdigráficas. Si $D$ es una digráfica y $r$ un entero no negativo, denotaremos por $r \cdot D$ el conjunto de $r$ copias isomorfas de $D$.

Si $S \subset V(D)$, la subdigráfica inducida $D\langle S\rangle$ se define como la digráfica con conjunto de vértices y conjunto de flechas

$$
\begin{aligned}
& V(D\langle S\rangle)=S, \\
& A(D\langle S\rangle)=\{u \rightarrow v \text { en } A(D): u, v \in S\},
\end{aligned}
$$

respectivamente. Un conjunto $S$ es acíclico si $D\langle S\rangle$ es acíclica y similarmente, un subconjunto de flechas de $D$ es acíclico (resp. libre de $\vec{C}_{3}$ ) si no contiene ciclos (resp. triángulos dirigidos). De manera más general, si $P$ es una digráfica dada y la digráfica $D$ no contiene a $P$ como subdigráfica, entonces diremos que $D$ es libre de $P$.

Una digráfica $D$ es fuertemente conexa si para todo par de vértices $u, v \in V(D)$, existe una $(u, v)$-trayectoria. Si solo garantizamos la existencia de una de las trayectorias $u \rightsquigarrow v$ o $v \rightsquigarrow u$, diremos que la digráfica es unilateral. Una digráfica $D$ es débilmente 
conexa si su subgráfica subyacente es conexa. El número de componentes conexas débiles de una digráfica $D$ se denota por $\omega(D)$.

Una gráfica es completa si para todo par de vértices, existe exactamente una arista entre ellos. Un torneo $T$ es una orientación de la gráfica completa. El torneo $T$ es regular si para toda $v \in V(T)$, se cumple que $d^{+}(v, T)=d^{-}(v, T)$. Similarmente el torneo $T$ es semiregular si

$$
\left|d^{+}(v, T)-d^{-}(v, T)\right|=1,
$$

para toda $v \in V(T)$. Un torneo de orden $n$ lo denotaremos por $T_{n}$. Si $T_{n}$ es regular entonces es de orden impar y si es semirregular, entonces es de orden par y lo denotaremos por $S R_{n}$. El torneo transitivo $T T_{n}$ es el torneo acíclico de orden $n$.

Una partición $P$ del conjunto de vértices de $D$ en $r$ partes (con $r \geq 1$ ), la denotaremos por

$$
P=P_{1}\left|P_{2}\right| \ldots \mid P_{r} .
$$

Si $\left|P_{i}\right|=1$ para alguna $i \in\{1,2, \ldots, r\}$, diremos que es una partición singular y por lo tanto diremos que el vértice $v \in P_{i}$ es un vértice singular. Si cada parte $P_{i}$ es no vacía, diremos que es una partición no degenerada.

Dada una partición $P$ de $V(D)$, una flecha $u \rightarrow v$ se dice que es interna (resp. externa) si $u$ y $v$ pertenecen a la misma clase (resp. distintas clases) de equivalencia de $P$. Así mismo $P$ es internamente (resp. externamente) acíclica (resp. libre de $\vec{C}_{3}$ ) si el conjunto de flechas internas (resp. externas) es acíclico (resp. libre de $\vec{C}_{3}$ ).

A la partición no degenerada $V(D)=X_{1}\left|X_{2}\right| \ldots \mid X_{r}$, le asociamos la función

$$
\varphi: V(D) \rightarrow\left\{X_{1}, X_{2}, \ldots, X_{r}\right\}
$$

que es una $r$-coloración que asigna a todo vértice $u \in X_{i} \subset V(D)$ el color $X_{i}$, es decir, $\varphi(u)=X_{i}$. En lo sucesivo, una $r$-coloración $\varphi$ será una función sobreyectiva, es decir, daremos por entendido que cada color se asigna al menos a un vértice. Una subdigráfica $D_{0} \subseteq D$ está bien coloreada si todo par de vértices vecinos en $D_{0}$ recibe distinto color. En general, si todo vértice de $D_{0}$ recibe exactamente un color distinto, entonces diremos que la coloración con $r$ colores es heterocromática y si todos los vértices de $D_{0}$ son del mismo color, diremos que es monocromática. Dado un vértice $v \in V(D)$, al suponer que $\varphi(v)=X_{i}$ con $i \in\{1,2, \ldots, r\}$, estaremos hablando indiferentemente del $i$-ésimo color o de la $i$-ésima clase cromática a la que pertenece.

Una subdigráfica $D_{0} \subseteq D$ es llamada generadora si $V(D)=V\left(D_{0}\right)$. Una subdigráfica $D_{0} \subseteq D$ es una $\vec{C}$-transversal lineal (resp. $\overrightarrow{C_{3}}$-transversal lineal) de $D$, si para todo ciclo dirigido (resp. triángulo dirigido) $\vec{C}$, se tiene que

$$
A\left(D_{0}\right) \cap A(\vec{C}) \neq \emptyset \text {. }
$$

El conjunto de $\vec{C}$-transversales lineales $\left(\vec{C}_{3}\right.$-transversales lineales) se denotará por $\operatorname{Tr}(D)\left(\right.$ resp. $\left.\operatorname{Tr}_{3}(D)\right)$. Notemos que $D_{0} \in \operatorname{Tr}(D)$ (resp. $\left.D_{0} \in \operatorname{Tr}_{3}(D)\right)$ si y solo si $D \backslash A\left(D_{0}\right)$ es acíclica (resp. libre de $\overrightarrow{C_{3}}$ ). 


\subsection{Inconexión acíclica e inconexión libre de $\vec{C}_{3}$}

En 1999, Víctor Neumann-Lara en su artículo [NL99], define la inconexión acíclica y la inconexión libre de triángulos, que junto con el número dicromático definido en [NL82] -del cual hablaremos en los capítulos posteriores-, miden la estructura cíclica de las digráficas.

El presente trabajo tiene como principal objetivo estudiar dichos parámetros asociados a digráficas muy especiales: los torneos.

Definición 1.1. La inconexión acíclica $\vec{\omega}(D)$ de una digráfica $D$, se define como el máximo número de componentes conexas (débiles) de la subdigráfica inducida de D, que se obtiene al borrar un conjunto acíclico de flechas, es decir

$$
\vec{\omega}(D)=\operatorname{máx}\{\omega(D \backslash F): F \subseteq A(D) \text { y } F \text { es acíclico }\} \text {. }
$$

Proposición 1.1 (Proposición 2.2 en [NL99]). Las siguientes son definiciones equivalentes de $\vec{\omega}(D)$ :

(i) $\operatorname{máx}\{\omega(D \backslash F): F \subseteq A(D)$ y $F$ es acíclico $\}$.

(ii) $\operatorname{máx}\left\{\omega\left(D_{0}\right): D_{0} \in \operatorname{Tr}(D)\right\}$.

(iii) La máxima cardinalidad de una partición de $V(D)$ externamente acíclica.

(iv) El máximo número de colores de una coloración de $V(D)$, que no produce ciclos dirigidos bien coloreados.

Demostración. (i) $\Rightarrow$ (ii). Sea $F \subseteq A(D)$ un conjunto acíclico de flechas tal que (i) alcanza su máximo. Sea $D_{0}=(V(D), A(D) \backslash F)$, entonces dado un ciclo dirigido $\vec{C}$, se tiene que $A\left(D_{0}\right) \cap A(\vec{C}) \neq \emptyset$, de lo contrario, si fuera vacío, se tendría que $A(\vec{C}) \subset F$ para todo ciclo dirigido $\vec{C}$. Esto es una contradicción al hecho que $F$ es acíclico. Luego $D_{0} \in \operatorname{Tr}(D)$ si $D_{0}$ es acíclica. Por lo tanto se tiene que

$$
\operatorname{máx}\{\omega(D \backslash F): F \subseteq A(D) \text { y } F \text { es acíclico }\}=\operatorname{máx}\left\{\omega\left(D_{0}\right): D_{0} \in \operatorname{Tr}(D)\right\} \text {. }
$$

(ii) $\Rightarrow$ (iii). Sea $r=\operatorname{máx}\left\{\omega\left(D_{0}\right): D_{0} \in \operatorname{Tr}(D)\right\}$, entonces existen $r$ componentes conexas que proporcionan una partición con $r$ clases de equivalencia de $A(D)$. Como $D \backslash A\left(D_{0}\right)$ es acíclica, entonces dicha partición es maximal y externamente acíclica.

(iii) $\Rightarrow$ (iv). Sea $P=P_{1}\left|P_{2}\right| \ldots \mid P_{r}$ una partición de $r$ partes no degenerada, maximal y externamente acíclica de $V(D)$. Sea $\varphi: V(D) \rightarrow\left\{X_{1}, X_{2}, \ldots, X_{r}\right\}$ una $r$-coloración definida por $\varphi\left(P_{i}\right)=X_{i}$ para toda $i \in\{1,2, \ldots, r\}$. Como $P$ es externamente acíclica, entonces ningún ciclo dirigido está bien coloreado. 
(iv) $\Rightarrow$ (i). Sea $\varphi: V(D) \rightarrow\left\{X_{1}, X_{2}, \ldots, X_{r}\right\}$ una $r$-coloración maximal de $V(D)$. Sea

$$
F=\left\{u \rightarrow v \text { en } A(D): u \in \varphi^{-1}\left(X_{i}\right), v \in \varphi^{-1}\left(X_{j}\right), i \neq j\right\},
$$

entonces $F$ es acíclico pues no hay ciclos bien coloreados y $r=\omega(D \backslash F)$ es máxima.

La siguiente definición es análoga a la definición 1.1 sobre inconexión acíclica, la cual no contempla todos los ciclos de la digráfica $D$, solo se restringe al análisis de los triángulos dirigidos. Ambos parámetros guardan propiedades muy cercanas, sobre todo en torneos, los cuales analizaremos más adelante.

Definición 1.2. La inconexión libre de triángulos (o de $\left.\vec{C}_{3}\right) \vec{\omega}_{3}(D)$ de una digráfica $D$, se define como el máximo número de componentes conexas (débiles) de la subdigráfica inducida de $D$, que se obtiene al borrar un conjunto de flechas libre de $\vec{C}_{3}$, es decir

$$
\vec{\omega}_{3}(D)=\operatorname{máx}\left\{\omega(D \backslash F): F \subseteq A(D) \text { y } F \text { es libre de } \vec{C}_{3}\right\} \text {. }
$$

Similarmente a la definición anterior, tenemos la siguiente.

Proposición 1.2 (Proposición 2.3 en [NL99]). Las siguientes son definiciones equivalentes de $\vec{\omega}_{3}(D)$ :

(i) $\operatorname{máx}\left\{\omega(D \backslash F): F \subseteq A(D)\right.$ y $F$ es libre de $\left.\vec{C}_{3}\right\}$.

(ii) $\operatorname{máx}\left\{\omega\left(D_{0}\right): D_{0} \in \operatorname{Tr}_{3}(D)\right\}$.

(iii) La máxima cardinalidad de una partición de $V(D)$ externamente libre de $\vec{C}_{3}$.

(iv) El máximo número de colores de una coloración de $V(D)$, que no produce triángulos dirigidos heterocromáticos.

Demostración. Similar a la proposición 1.1.

En lo sucesivo diremos que una partición es óptima, si no es posible aumentar el número de clases de equivalencia. Similarmente una coloración es óptima o maximal si realiza la inconexión acíclica (resp. la inconexión libre de $\overrightarrow{C_{3}}$ ) y es imposible agregar un nuevo color sin inducir ciclos dirigidos bien coloreados (resp. triángulos dirigidos heterocromáticos).

Para propósitos de este trabajo, utilizaremos las definiciones de inconexión acíclica y de inconexión libre de $\overrightarrow{C_{3}}$ en términos de coloraciones, esto es, en su forma equivalente (iv) de las proposiciones 1.1 y 1.2 respectivamente.

Observación 1.1. Para toda digráfica D se cumple que

$$
\vec{\omega}(D) \leq \vec{\omega}_{3}(D)
$$


En la figura 1.1 mostramos dos ejemplos de digráficas y una coloración que proporciona la inconexión acíclica para cada una de ellas. Notemos que para la digráfica $D$ del inciso a) que $\vec{\omega}(D)=4$ y esta coloración es libre de $\vec{C}_{3}$. Más aún, es imposible agregar un nuevo color sin inducir $\overrightarrow{C_{3}}$ heterocromáticos, por lo que $\vec{\omega}(D)=\overrightarrow{\omega_{3}}(D)=4$.

Para el ciclo $\vec{C}_{9}$ del inciso b), es necesario colorear dos vértices adyacentes del mismo color para que la coloración sea libre de ciclos bien coloreados. Así $\vec{\omega}\left(\vec{C}_{9}\right)=8$, sin embargo, claramente $\vec{C}_{9}$ no contiene triángulos, por lo que si asignamos un color distinto a cada vértice, entonces dicha coloración es libre de $\vec{C}_{3}$, por lo que $\vec{\omega}_{3}\left(\vec{C}_{9}\right)=9$ y por lo tanto $\vec{\omega}\left(\vec{C}_{9}\right)<\vec{\omega}_{3}\left(\vec{C}_{9}\right)$.

En general para un ciclo $\vec{C}_{n}$ se tiene que

$$
n-1=\vec{\omega}\left(\vec{C}_{n}\right)<\vec{\omega}_{3}\left(\vec{C}_{n}\right)=n
$$

lo que muestra que la desigualdad $\vec{\omega}(D) \leq \vec{\omega}_{3}(D)$ puede ser estricta.

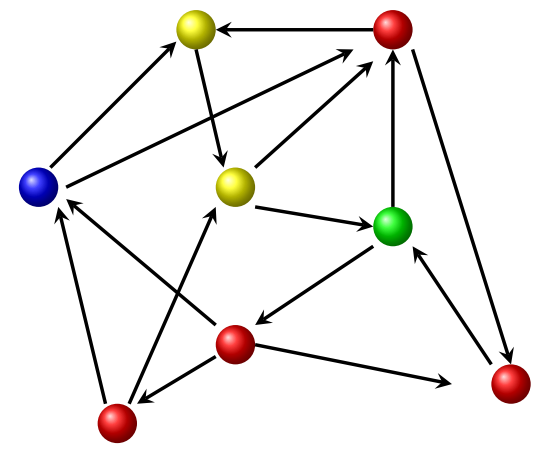

a) Una digráfica $D$.

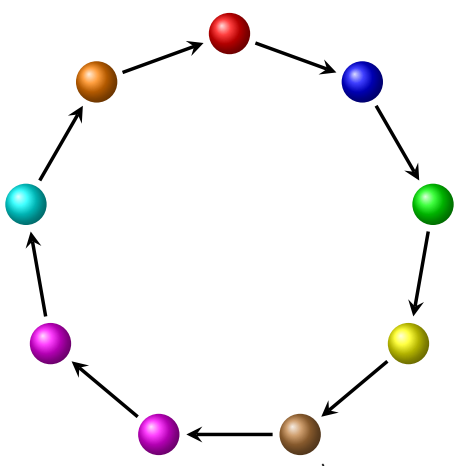

b) El ciclo $\vec{C}_{9}$

Figura 1.1: Dos ejemplos de digráficas y una coloración óptima.

Si $D$ es una digráfica, $S C(D)$ denotará el conjunto de componentes conexas (débiles) de $D$.

Proposición 1.3 (Proposición 3.1 en [NL99]). La inconexión acíclica (resp. libre de $\overrightarrow{C_{3}}$ ) de una digráfica $D$ es la suma de la inconexión acíclica (resp. libre de $\overrightarrow{C_{3}}$ ) de cada una de sus componentes conexas, es decir

$$
\vec{\omega}(D)=\sum_{\alpha \in S C(D)} \vec{\omega}(\alpha) \quad\left(\text { resp. } \overrightarrow{\omega_{3}}(D)=\sum_{\alpha \in S C(D)} \overrightarrow{\omega_{3}}(\alpha)\right)
$$

Demostración. La demostración es consecuencia inmediata de las definiciones de $\vec{\omega}$ y $\overrightarrow{\omega_{3}}$ respectivamente. 


\subsection{Torneos}

Recordemos que un torneo se define a partir de la gráfica completa, a la cual le hemos asignado alguna orientación. Los torneos son una clase especial de digráficas ampliamente estudiados, cuyas áreas de aplicaciones comprenden por ejemplo: comparación de experimentos, dominación de sociedades animales, redes y comunicaciones, población y votaciones, por mencionar algunas.

El lector interesado en otros resultados sobre los torneos, puede consultar [R04], [BR79] o bien [MJW68]. Este último es un clásico y una excelente referencia de la teoría que rodea este tema. El lector también puede consultar [Mc], donde encontrará un banco de datos de torneos regulares, semirregulares, entre otros.

Primero comenzaremos con un lema muy conocido de Alspach, el cual se puede encontrar en [A67], sobre ciclos de cualquier longitud contenidos en un torneo, pero del cual solo nos interesa el caso particular de los triángulos dirigidos.

Lema 1.1 (Alspach). Si $T$ es un torneo regular, entonces toda flecha de $A(T)$ está en un triángulo dirigido.

Demostración. Supongamos que $u \rightarrow v$ es una flecha en el torneo $T$ de orden $n$. Como $u, v \notin N^{+}(v)$ y $u, v \notin N^{-}(u)$, entonces

$$
\begin{aligned}
n-2 & \geq\left|N^{+}(v) \cup N^{-}(u)\right| \\
& =d^{+}(v)+d^{-}(u)-\left|N^{+}(v) \cap N^{-}(u)\right| \\
& =n-1-\left|N^{+}(v) \cap N^{-}(u)\right|,
\end{aligned}
$$

así, $\left|N^{+}(v) \cap N^{-}(u)\right| \geq 1$, por lo que $u \rightarrow v$ está en al menos un triángulo dirigido.

Corolario 1.1. Si $T$ es un torneo regular, entonces toda partición externamente libre de $\overrightarrow{C_{3}}$ de $V(T)$ tiene a lo más una clase de equivalencia singular.

Demostración. Supongamos por contradicción que existe una partición de $V(T)$, con $r \geq 3$ partes

$$
P=P_{1}\left|P_{2}\right| \ldots \mid P_{r}
$$

con al menos dos clases de equivalencia singulares. Sean $P_{i}=\{u\}$ y $P_{j}=\{v\}$ dos partes singulares, con $i, j \in\{1,2, \ldots, r\}$ tal que $i \neq j$. Supongamos sin pérdida de generalidad que $u \rightarrow v$, entonces por el lema 1.1 , se tiene que $u \rightarrow v$ está en un triángulo dirigido, es decir existe $w \in P_{k}, k \notin\{i, j\}$ tal que

$$
\overrightarrow{C_{3}}: w \rightarrow u \rightarrow v \rightarrow w,
$$

que es una contradicción al hecho de ser una partición externamente libre de $\vec{C}_{3}$.

Si traducimos el corolario anterior al lenguaje de coloraciones, obtenemos que para un torneo $T$, en toda coloración de sus vértices, existe a lo más una clase cromática singular. 
Observación 1.2. Para todo torneo T se cumple que

$$
2 \leq \vec{\omega}(T) \leq \vec{\omega}_{3}(T)
$$

Claramente siempre es posible colorear los vértices de un torneo con dos colores, sin inducir ciclos ni triángulos bien coloreados. Para los torneos cuya inconexión libre de $\overrightarrow{C_{3}}$ es igual a la cota inferior, tenemos la siguiente definición que jugará un papel importante en los capítulos siguientes.

Definición 1.3. Diremos que un torneo $T$ es tenso si $\overrightarrow{\omega_{3}}(T)=2$.

En [NL99], Víctor Neumann-Lara proporciona el primer ejemplo -y único en la literatura- de un torneo no regular $T$ de orden 9 , que cumple con la desigualdad de la observación 1.2 en forma estricta, esto es, demuestra que $\vec{\omega}(T)=2$ y $\vec{\omega}_{3}(T)=3$. Esta observación proporciona el primer problema que resolvemos en esta tesis.

Problema 1.1. ¿Qué tan grande puede ser la diferencia

$$
\vec{\omega}_{3}(T)-\vec{\omega}(T) ?
$$

Para realizar la inconexión acíclica, necesitamos al menos dos colores, que es el mínimo número posible para obtener esta propiedad, por lo que tiene sentido pensar que si $\overrightarrow{\omega_{3}}$ es el máximo número, entonces toda $\left(\overrightarrow{\omega_{3}}+1\right)$-coloración produce al menos un triángulo dirigido heterocromático. La siguiente definición resume lo anterior para ciclos dirigidos en general.

Definición 1.4. Sea $D$ una digráfica y definamos

$$
\vec{\omega}^{+}(D)=\vec{\omega}(D)+1 \quad\left(\operatorname{resp} . \quad \vec{\omega}_{3}^{+}(D)=\vec{\omega}_{3}(D)+1\right),
$$

entonces $\vec{\omega}^{+}(D)$ (resp. $\vec{\omega}_{3}^{+}(D)$ ) es el mínimo número de colores necesarios, de tal forma que para toda $\vec{\omega}^{+}(D)$-coloración (resp. $\vec{\omega}_{3}^{+}(D)$-coloración) de $V(D)$, existe al menos un ciclo dirigido bien coloreado (resp. $\overrightarrow{C_{3}}$ heterocromático).

Notemos que en la definición 1.4, $\vec{\omega}^{+}(D)$ y $\vec{\omega}_{3}^{+}(D)$ son formas alternativas de definir la inconexión acíclica y la inconexión libre de $\overrightarrow{C_{3}}$ respectivamente y de la misma definición, se sigue inmediatamente que un torneo $T$ es tenso si y solo si $\overrightarrow{\omega_{3}}(T)=2$, si y solo si $\vec{\omega}_{3}^{+}(T)=3$.

Los orígenes del concepto de tensión en gráficas, se remontan al artículo [ABNL92] de 1992, en el que J. L. Arocha, J. Bracho y el mismo V. Neumann-Lara lo definen en términos de hipergráficas como sigue.

Recordemos que una hipergráfica $H$ se define sobre un conjunto de vértices $V(H)$ e hiperaristas $E(H)$, donde $E(H)$ es un conjunto de subconjuntos de $V(H)$. Si consideramos que las aristas de una gráfica, son subconjuntos de cardinalidad dos, entonces una hipergráfica es la generalización natural del concepto de gráfica al tomar subconjuntos de cualquier cardinalidad. No es difícil probar que una gráfica $G$ es conexa si y 
solo si para toda 2-coloración de sus vértices, existe una arista con extremos de distinto color, es decir induce una arista heterocromática. Si consideramos a las aristas de una gráfica como un ciclo de longitud dos, entonces toda 2-coloración deja siempre un ciclo bien coloreado y podemos pensar a la inconexión acíclica como una generalización del concepto de conexidad en hipergráficas.

Una 3-hipergráfica uniforme (o simplemente una 3-gráfica) $H$, es la hipergráfica definida sobre un conjunto de vértices $V(H)$ y un conjunto de hiperaristas (llamadas 3 -aristas)

$$
E(H)=\{S \subset V(H):|S|=3\}
$$

El número heterocromático $h c(H)$ es el mínimo número de colores $r$ de tal manera que toda $r$-coloración de los vértices de $H$, induce al menos una 3-arista heterocromática. Decimos que $H$ es tensa si $h c(H)=3$ (véase [ABNL92, ABNL95] para más detalles).

Para un torneo $T, \vec{\omega}_{3}+(T)$ es el número heterocromático $h c\left(H_{T}\right)$ de la 3-gráfica $H_{T}=(V, E)$ que se obtiene de $T$ al definir $V\left(H_{T}\right)=V(T)$ y al conjunto de 3-aristas como

$$
E\left(H_{T}\right)=\left\{S \subseteq V(T): T\langle S\rangle \cong \overrightarrow{C_{3}}\right\} .
$$

Observemos que $T$ es tenso si y solo si $H_{T}$ es tensa, es decir,

$$
\vec{\omega}_{3}^{+}(T)=h c\left(H_{T}\right)=3
$$

Denotaremos por $\mathbb{Z}_{n}$ al conjunto de enteros módulo $n$ y recordemos que en teoría aditiva de números (véase [N96] para mayor detalle), dados los conjuntos $A, B \subseteq \mathbb{Z}_{n}$, entonces

$$
\begin{aligned}
A+B & =\{a+b: a \in A, b \in B\} \\
-A & =\{-a: a \in A\} .
\end{aligned}
$$

Definición 1.5. Sea $J \subset \mathbb{Z}_{n} \backslash\{0\}$, la digráfica circulante $\vec{C}_{n}(J)$ se define sobre los conjuntos

$$
\begin{aligned}
V\left(\vec{C}_{n}(J)\right) & =\mathbb{Z}_{n}, \\
A\left(\vec{C}_{n}(J)\right) & =\left\{i \rightarrow j: i, j \in \mathbb{Z}_{n}, j-i \in J\right\}
\end{aligned}
$$

Notemos que si $J=\{1\}$, entonces $\vec{C}_{n}(J) \cong \vec{C}_{n}(1)$ es simplemente el ciclo dirigido $\vec{C}_{n}$ y si $J \subset \mathbb{Z}_{2 n+1} \backslash\{0\}$ tal que $|J|=n$, entonces $\vec{C}_{2 n+1}(J)$ es un torneo circulante si y solo si $|\{j,-j\} \cap J|=1$ para toda $j \in \mathbb{Z}_{2 n+1} \backslash\{0\}$. El conjunto $J$ es comúnmente llamado conjunto de saltos.

Sea $S \subseteq\{1,2, \ldots, n\}$. Definimos al torneo circulante

$$
\vec{C}_{2 n+1}\langle S\rangle=\vec{C}_{2 n+1}((\{1,2, \ldots, n\} \backslash S) \cup(-S)),
$$


es decir, $S$ representa el conjunto que flechas que se dibujarán en dirección opuesta. Si $S=\emptyset$, entonces el torneo regular circulante $\vec{C}_{2 n+1}(\{1,2, \ldots, n\})=\vec{C}_{2 n+1}\langle\emptyset\rangle$ lo llamaremos simplemente el torneo cíclico. Es bien conocido que los torneos obtenidos de esta forma, son regulares y que su grupo de automorfismos actúa transitivamente en el conjunto de vértices, esto es, para toda $j \in \mathbb{Z}_{2 n+1} \backslash\{0\}$, la función

$$
\phi: V(T) \rightarrow V(T),
$$

definida por $\phi(i)=i+j($ mód $2 n+1)$ manda el vértice $i \in V\left(\vec{C}_{2 n+1}(J)\right)$ al vértice $i+j$, para alguna $j \in V(T)$. No es difícil convencerse que esta biyección preserva vecindades $\mathrm{y}$ adyacencias.

La figura 1.2 muestra los dos torneos regulares circulantes de orden 7

$$
\vec{C}_{7}(\{1,2,3\}) \cong \vec{C}_{7}\langle\emptyset\rangle \quad \text { y } \quad \vec{C}_{7}(\{1,2,4\}) \cong \vec{C}_{7}\langle 3\rangle
$$

que son únicos, salvo isomorfismos. El torneo circulante $\vec{C}_{7}(\{1,2,4\}) \cong \vec{C}_{7}\langle 3\rangle$ es mejor conocido como el torneo de Paley de orden 7 o de residuos cuadráticos de $\mathbb{Z}_{7}$, ya que precisamente su conjunto de saltos es el conjunto de residuos cuadráticos de $\mathbb{Z}_{7}$.

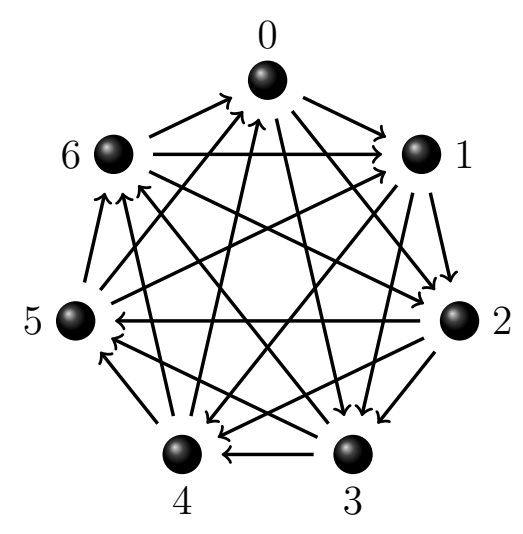

a) $\vec{C}_{7}(\{1,2,3\}) \cong \vec{C}_{7}\langle\emptyset\rangle$.

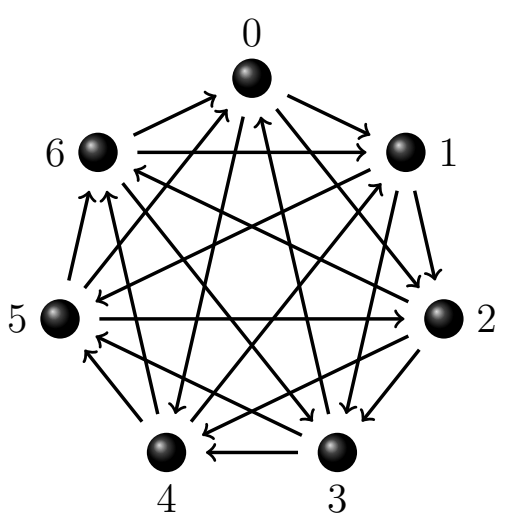

b) $\vec{C}_{7}(\{1,2,4\}) \cong \vec{C}_{7}\langle 3\rangle$.

Figura 1.2: Torneos regulares circulantes de orden 7.

\subsection{Sumas de Zykov}

A partir de dos o más digráficas dadas, es posible operarlas de tal manera, que podemos construir nuevas digráficas a partir de las digráficas originales. Es esta sección abordamos una de dichas operaciones: la suma de Zykov o también llamada $X$-unión (véase [IKH11]), la cual tiene propiedades interesantes y fundamentales en el estudio de la inconexión acíclica y la inconexión libre de $\overrightarrow{C_{3}}$. Más adelante veremos que mediante esta operación, de cierta forma se «heredan» algunas propiedades, entre ellas, las coloraciones de las digráficas originales a la digráfica resultante de una suma de Zykov. 
Definición 1.6. Sean $B$ una digráfica con conjunto de vértices $V(B)=\{1,2, \ldots, n\}$ y $\alpha=\left\{\alpha_{i}\right\}_{i \in V(B)}$ una familia no vacía de digráficas ajenas dos a dos, entonces la suma de Zykov $B[\alpha]=B\left[\alpha_{1}, \alpha_{2}, \ldots, \alpha_{n}\right]$ es la digráfica definida por

$$
\begin{aligned}
& V(B[\alpha])=\bigcup_{i \in V(B)} V\left(\alpha_{i}\right) y \\
& A(B[\alpha])=\bigcup_{i \in V(B)} A\left(\alpha_{i}\right) \cup\left\{u \rightarrow v: u \in V\left(\alpha_{i}\right), v \in V\left(\alpha_{j}\right), i \rightarrow j \text { está en } A(B)\right\} .
\end{aligned}
$$

Si los miembros de la familia $\alpha$ no son ajenos dos a dos, entonces pueden sustituirse por copias isomorfas de los mismos de tal manera que la familia esté constituida por miembros ajenos dos a dos. A la digráfica $B$ la llamaremos base de la suma de Zykov y diremos que $B[\alpha]$ es una suma de Zykov no trivial si $\left|V\left(\alpha_{i}\right)\right| \geq 2$, para alguna $i \in V(B)$.

Definición 1.7. La función $\pi: V\left(B\left[\alpha_{1}, \alpha_{2}, \ldots, \alpha_{n}\right]\right) \rightarrow V(B)$ definida por $\pi\left(\alpha_{i}\right)=i$ para toda $i \in V(B)$ es un epimorfismo reflexivo, a la cual nos referiremos como la proyección natural de $B[\alpha]$ sobre $B$.

Definición 1.8. Si $\alpha_{i} \cong D$ para alguna digráfica $D$ y para todo $i \in V(B)$, entonces $B[D]$ es simplemente la composición (o producto lexicográfico en [IKH11]) de $B$ y D. Si una digráfica no es isomorfa a composición alguna, entonces se dirá simple. Más aún, si una digráfica no es isomorfa a una suma de Zykov no trivial, entonces diremos que es prima o no factorizable.

El siguiente lema es fácil de demostrar, pues se sigue directamente de la definición de una suma de Zykov.

Lema 1.2. Sean $B$ una digráfica y $\alpha=\left\{\alpha_{i}\right\}_{i \in V(B)}$ una familia no vacía de digráficas. Si $B$ y toda $\alpha_{i}$ son acíclicas, entonces $B[\alpha]$ es también acíclica.

El siguiente teorema es de gran importancia para el objetivo de este trabajo, el cual proporciona una forma de obtener la inconexión acíclica y la inconexión libre de $\overrightarrow{C_{3}}$ en una digráfica dada. Sea $D^{\circ}$ la subdigráfica inducida de una digráfica $D$ obtenida al borrar los vértices aislados.

Teorema 1.1 (Teorema 3.4 en [NL99]). Sean B una digráfica y $\alpha=\left\{\alpha_{i}\right\}_{i \in V(B)}$ una familia no vacía de digráficas, entonces

$$
\begin{gathered}
\vec{\omega}(B[\alpha])=\operatorname{máx}_{W \in \operatorname{Tr}(B)}\left\{\omega\left(W^{\circ}\right)+\sum_{j \in J} \vec{\omega}\left(\alpha_{j}\right)\right\} \\
\left(\text { resp. } \overrightarrow{\omega_{3}}(B[\alpha])=\operatorname{máx}_{W \in T r_{3}(B)}\left\{\omega\left(W^{\circ}\right)+\sum_{j \in J} \vec{\omega}_{3}\left(\alpha_{j}\right)\right\}\right),
\end{gathered}
$$

donde $J=V(B) \backslash V\left(W^{\circ}\right)$. 
Demostración. Este teorema es un caso particular del teorema $1.2^{1}$ que se probará más adelante.

El siguiente corolario proporciona un resultado interesante, el cual señala que si una digráfica $D$ es isomorfa a una suma de Zykov no trivial, entonces $\vec{\omega}(D) \geq 3$ y $\overrightarrow{\omega_{3}}(D) \geq 3$, es decir, una suma de Zykov no es tensa. Esto nos será de gran utilidad más adelante para caracterizar los torneos regulares tensos.

Corolario 1.2. Sea $B[\alpha]$ una suma de Zykov no trivial, entonces $\vec{\omega}(B[\alpha]) \geq 3$ (resp. $\left.\overrightarrow{\omega_{3}}(B[\alpha]) \geq 3\right)$.

Demostración. Sea $B[\alpha]$ como en el teorema 1.1. Notemos que para calcular la inconexión acíclica de una digráfica dada, se necesitan al menos dos colores. Como $\alpha$ es una familia no trivial, entonces existe $j \in J$ tal que $\left|V\left(\alpha_{j}\right)\right| \geq 2$ y así $\vec{\omega}\left(\alpha_{j}\right) \geq 2$. Notemos además que dada $W \in \operatorname{Tr}(B)$, entonces $\omega\left(W^{\circ}\right) \geq 1$, por lo que $\omega\left(W^{\circ}\right)+\vec{\omega}\left(\alpha_{j}\right) \geq 3$ y así se obtiene lo deseado.

Se demuestra de manera similar para $\overrightarrow{\omega_{3}}$.

Como ya lo hemos mencionado, de alguna manera se heredan algunas coloraciones de las digráficas originales a la suma de Zykov. Para entender esto, tenemos la definición y la observación siguientes que nos ayudarán a describir esta situación.

Definición 1.9. Una digráfica $D$ se dice $\vec{\omega}$-aguda (resp. $\vec{\omega}_{3}$-aguda) si

(i) existe una r-coloración óptima de $V(D)$,

(ii) dicha coloración tiene exactamente una clase cromática singular y

(iii) no existe otra r-coloración que fija más de una clase cromática singular.

Un ejemplo inmediato de una digráfica que es $\vec{\omega}$-aguda y $\overrightarrow{\omega_{3}}$-aguda es el triángulo $\overrightarrow{C_{3}}$. Los ejemplos dados en la figura 1.1 de la página 6 , no son $\vec{\omega}$-agudos ni $\overrightarrow{\omega_{3}}-$ agudos, ya que las coloraciones mostradas contienen más de una clase cromática singular.

Proposición 1.4 (Proposición 3.6 en [NL99]). Si B es $\vec{\omega}$-aguda (resp. $\vec{\omega}_{3}$-aguda), entonces tenemos las siguientes propiedades:

(i) Si D es una digráfica, entonces

$$
\vec{\omega}(B[D])=\vec{\omega}(B)+\vec{\omega}(D)-1 \quad\left(\operatorname{resp} . \vec{\omega}_{3}(B[D])=\vec{\omega}_{3}(B)+\vec{\omega}_{3}(D)-1\right) .
$$

(ii) Si $D$ es $\vec{\omega}$-aguda (resp. $\vec{\omega}_{3}$-aguda), entonces $B[D]$ es también $\vec{\omega}$-aguda (resp. $\left.\vec{\omega}_{3}-a g u d a\right)$.

Demostración. En la proposición 1.6 de la página 18, demostramos una generalización de esta proposición.

\footnotetext{
${ }^{1}$ página 16
} 
La siguiente observación describe la manera en la que las digáficas $B$ y $D$ «heredan» una coloración óptima a la composición $B[D]$.

Observación 1.3. Sean $B$ una digráfica $\vec{\omega}$-aguda (resp. $\overrightarrow{\omega_{3}}-$ aguda) y D una digráfica tales que $\vec{\omega}(B)=s$ y $\vec{\omega}(D)=r$ (resp. $\overrightarrow{\omega_{3}}(B)=s$ y $\left.\overrightarrow{\omega_{3}}(D)=r\right)$. Sea

$$
\varphi_{B}: V(B) \rightarrow\left\{X_{1}, X_{2}, \ldots, X_{s}\right\}
$$

una s-coloración que sin pérdida de generalidad, supongamos que proporciona la clase cromática singular $X_{s}$. Similarmente, sea

$$
\varphi_{D}: V(D) \rightarrow\left\{Y_{1}, Y_{2}, \ldots, Y_{r}\right\}
$$

una $r$-coloración para $V(D)$. Entonces una $(s+r-1)$-coloración óptima para la composición $B[D]$,

$$
\varphi_{B[D]}: V(B[D]) \rightarrow\left\{X_{1}, X_{2}, \ldots, X_{s-1}, Y_{1}, Y_{2}, \ldots, Y_{r}\right\}
$$

referida en la proposición 1.4 , se obtiene de la siguiente forma.

Denotemos por $D_{v}$ a la copia isomorfa de la digráfica $D$ en la composición $B[D]$, tal que $\pi\left(D_{v}\right)=v \in V(B)$, donde $\pi$ es la proyección natural $\pi: V(B[D]) \rightarrow V(B)$ dada en la definición 1.7 de la página 11.

(i) Si $v \in V(B)$ es el vértice asociado a la clase cromática singular $X_{s}$, entonces $\varphi_{B[D]}(u)=Y_{j}$ para toda $u \in V\left(D_{v}\right)$ y toda $j \in\{1,2, \ldots, r\}$.

(ii) Si $v \in V(B)$ no es el vértice singular y $\varphi_{B}(v)=X_{i}$, entonces $\varphi_{B[D]}(u)=X_{i}$ para toda $u \in V\left(D_{v}\right)$ y toda $i \in\{1,2, \ldots, s-1\}$.

Notemos que si (i) se cumple, entonces indica que todo vértice de la copia $D_{v}$, conserva una coloración isomorfa a la coloración $\varphi_{D}$ original en D. En cambio, si (ii) se cumple entonces cada vértice $u \in D_{v}$ se colorea del mismo color que tenía originalmente el vértice $v \in V(B)$ en la coloración $\varphi_{B}$.

Para ilustrar la observación anterior, sea la composición de $\overrightarrow{C_{3}}: v \rightarrow v^{\prime} \rightarrow v^{\prime \prime} \rightarrow v$ con $\overrightarrow{C_{3}}: 0 \rightarrow 1 \rightarrow 2 \rightarrow 0$ de la figura 1.3. Es claro que ambos torneos tienen inconexión acíclica e inconéxion libre de $\overrightarrow{C_{3}}$ igual a dos y tienen una 2-coloración como se muestra en la misma figura. Por la observación 1.3, una 3-coloración se puede dar al conservar la coloración original para una copia de $\vec{C}_{3}: 0 \rightarrow 1 \rightarrow 2 \rightarrow 0$ asociada al vértice singular $v$ y a los conjuntos de vértices $\left\{0^{\prime}, 1^{\prime}, 2^{\prime}\right\}$ y $\left\{0^{\prime \prime}, 1^{\prime \prime}, 2^{\prime \prime}\right\}$ se les asigna el mismo color que tenían asignados los vértices $v^{\prime} \mathrm{y} v^{\prime \prime}$ originalmente.

Haciendo uso de esta observación, podemos generalizar esta idea para un número arbitrario de digráficas. Tenemos el siguiente corolario. 

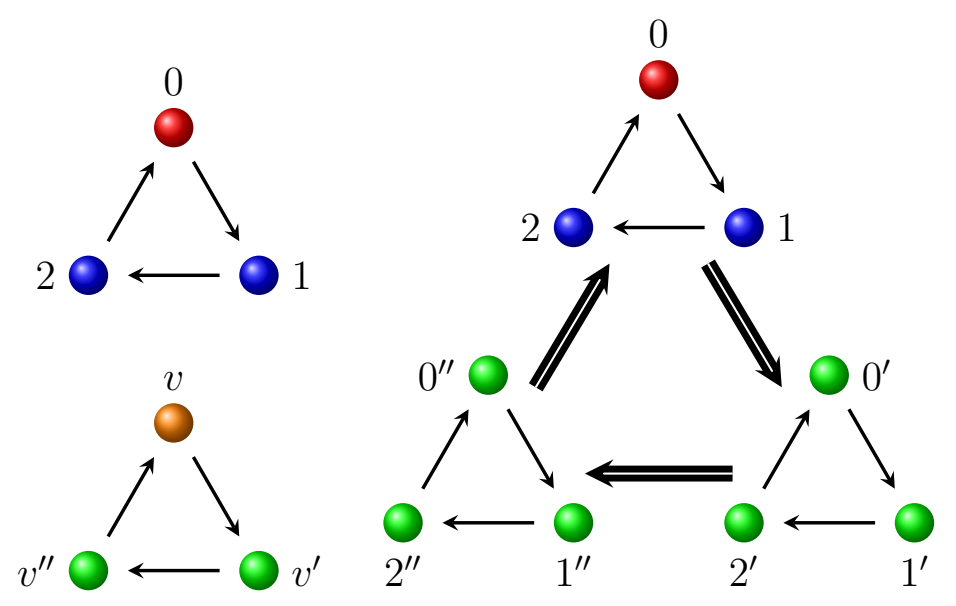

Figura 1.3: El torneo $\overrightarrow{C_{3}}\left[\overrightarrow{C_{3}}\right]$ y una coloración heredada.

Corolario 1.3. Sea $\left\{D_{i}\right\}_{i \in\{1,2, \ldots, r\}}$ una familia no vacía de digráficas $\vec{\omega}$-agudas (resp. $\overrightarrow{\omega_{3}}-$ agudas), entonces

$$
\begin{gathered}
\vec{\omega}\left(D_{1}\left[D_{2}\left[\ldots D_{r-1}\left[D_{r}\right]\right] \ldots\right]\right)=\sum_{i=1}^{r} \vec{\omega}\left(D_{i}\right)-(r-1) \\
\left(\operatorname{resp} . \vec{\omega}_{3}\left(D_{1}\left[D_{2}\left[\ldots D_{r-1}\left[D_{r}\right]\right] \ldots\right]\right)=\sum_{i=1}^{r} \vec{\omega}_{3}\left(D_{i}\right)-(r-1)\right) .
\end{gathered}
$$

Demostración. Se sigue por inducción a partir de la proposición 1.4 y la observación 1.3 .

Los siguientes lemas nos serán de mucha utilidad en los capítulos posteriores, cuando hablemos de composiciones o sumas de Zykov de torneos regulares.

Lema 1.3. Sea $T$ un torneo isomorfo a una suma de Zykov $B[\alpha]$. Si $T$ es regular entonces todo elemento de la familia $\alpha$ es un torneo regular.

Demostración. Si $B[\alpha]$ es trivial, entonces es claro que se cumple el lema. Supongamos que $\alpha=\left\{\alpha_{1}, \alpha_{2}, \ldots, \alpha_{|B|}\right\}$ es una familia no trivial y sin pérdida de generalidad supongamos que $\alpha_{1}$ es un elemento no trivial de la familia. Sean $v_{1}, v_{2} \in V\left(\alpha_{1}\right)$. Como es una suma de Zykov, se tiene que

$$
N^{+}\left(v_{1}, T\left\langle V(T) \backslash V\left(\alpha_{1}\right)\right\rangle\right)=N^{+}\left(v_{2}, T\left\langle V(T) \backslash V\left(\alpha_{1}\right)\right\rangle\right),
$$

por lo que

$$
d^{+}\left(v_{1}, T\left\langle V(T) \backslash V\left(\alpha_{1}\right)\right\rangle\right)=d^{+}\left(v_{2}, T\left\langle V(T) \backslash V\left(\alpha_{1}\right)\right\rangle\right) .
$$

Como $T$ es regular, finalmente concluimos que $d^{+}\left(v_{1}, \alpha_{1}\right)=d^{+}\left(v_{2}, \alpha_{1}\right)$ y así todo miembro de la familia $\alpha$ tiene que ser regular. 
El recíproco del lema anterior no es cierto, ya que la regularidad del torneo final $T$ depende de la regularidad de las componentes de la suma de Zykov y de tomar una base apropiada para la misma. El siguiente lema nos proporciona otro caso cuando la suma de Zykov es regular.

Lema 1.4. Sea $T=B[\alpha]$. Si $B$ es un torneo regular y todo miembro de la familia $\alpha$ es regular y del mismo orden, entonces $T$ es regular.

Demostración. Si todo torneo de la familia $\alpha$ es $r$-regular y la base $B$ es $s$-regular, es claro que la suma de Zykov es $(r \cdot s)$-regular.

\subsection{Generalización del concepto de inconexión acícli- ca y libre de $\vec{C}_{3}$}

Las siguientes definiciones nos ayudarán a generalizar el concepto de inconexión acíclica e inconexión libre de $\overrightarrow{C_{3}}$, con la finalidad de demostrar un teorema dado en [NL99] por Neumann-Lara para el cálculo de la inconexión acíclica y la inconexión libre de $\overrightarrow{C_{3}}$ de torneos.

Sean $J$ un conjunto de índices y $Q=\left\{Q_{i}\right\}_{i \in J}$ un conjunto no vacío de digráficas conexas. Una digráfica $D$ se dirá una $Q$-digráfica si $D \cong Q_{i}$ para alguna $Q_{i} \in Q$. Un conjunto de flechas $S$ de una digráfica $D$ se dirá libre de $Q$, si $D$ no contiene una $Q$-subdigráfica $D_{0}$ tal que $A\left(D_{0}\right) \subseteq S$.

Una subdigráfica generadora $W$ de $D$ es una $W$-flecha transversal de $D$, si para toda $Q$-subdigráfica $\Gamma$ de $D$, se tiene que $A\left(D_{0}\right) \cap A(\Gamma) \neq \emptyset$. Al conjunto de $Q$-flechas transversales de $D$ se denotará por $\operatorname{Tr}_{Q}(D)$. Es claro que $D_{0} \in \operatorname{Tr}_{Q}(D)$ si y solo si $D \backslash A\left(D_{0}\right)$ es libre de $Q$.

Diremos que el conjunto $Q$ es adecuado, si siempre que $D\left[\left\{\alpha_{i}\right\}_{i \in V(D)}\right]$ sea libre de $Q$, entonces $D$ y cada $\alpha_{i}$ son libres de $Q$.

Definición 1.10. La inconexión libre de $Q$ de una digráfica $D$ se define como

$$
\vec{\omega}(D, Q)=\operatorname{máx}\{\omega(D \backslash F): F \subseteq A(D), F \text { es libre de } Q\} \text {. }
$$

Notemos que si $D_{0}$ es una subdigráfica generadora de $D$, entonces

$$
\vec{\omega}(D, Q) \leq \vec{\omega}\left(D_{0}, Q\right)
$$

La siguiente proposición es una generalización de las proposiciones 1.1 y 1.2 de las

páginas 4 y 5 respectivamente, sobre inconexión acíclica e inconexión libre de $\vec{C}_{3}$, al hacer $Q=\left\{\vec{C}_{n}\right\}_{n \geq 3}$ y $Q=\vec{C}_{3}$ respectivamente.

Proposición 1.5. Las siguientes son definiciones equivalentes de $\vec{\omega}(D, Q)$ : 
(i) máx $\{\omega(D \backslash F): F \subseteq A(D)$, $F$ es libre de $Q\}$.

(ii) $\operatorname{máx}\left\{\omega\left(D_{0}\right): D_{0} \in \operatorname{Tr}_{Q}(D)\right\}$.

(iii) La máxima cardinalidad de una partición de $V(D)$ externamente libre de $Q$.

(iv) El máximo número de colores de una coloración de $V(D)$ tal que no produce $Q$-subdigráficas bien coloreadas.

Demostración. (i) $\Rightarrow$ (ii). Sea $F \subseteq A(D)$ tal que $D\langle F\rangle$ es libre de $Q$ y donde (i) alcanza su máximo. Sea $D_{0}=D \backslash F$, entonces dada una $Q$-subdigráfica $\gamma$, se tiene que $A\left(D_{0}\right) \cap A(\gamma) \neq \emptyset$, de lo contrario si fuera vacío, $V(\gamma) \subset F$ para toda $Q$-subdigráfica $\gamma$, que es una contradicción al hecho que $F$ es libre de $Q$. Luego $D_{0} \in \operatorname{Tr}_{Q}(D)$ si y solo si $D_{0}$ es libre de $Q$.

(ii) $\Rightarrow$ (iii). Sea $r=\operatorname{máx}\left\{\omega\left(D_{0}\right): D_{0} \in \operatorname{Tr}_{Q}(D)\right\}$, entonces existen $r$ componentes conexas que proporcionan una partición en $r$ clases de equivalencia de $A(D)$, la cual es libre de $Q$. Como $D \backslash A\left(D_{0}\right)$ es libre de $Q$, entonces dicha partición es externamente libre de $Q$.

(iii) $\Rightarrow$ (iv). Sea $P=P_{1}\left|P_{2}\right| \ldots \mid P_{r}$ una partición maximal de $V(D)$ en $r$ partes externamente libre de $Q$. Sea $\varphi: V(D) \rightarrow\left\{X_{1}, X_{2}, \ldots, X_{r}\right\}$ una $r$-coloración libre de $Q$, definida por $\varphi\left(P_{i}\right)=X_{i}$. Como $P$ es externamente libre de $Q$, entonces ninguna $Q$-subdigráfica está bien coloreada.

(iv) $\Rightarrow$ (i). Sea $\varphi: V(D) \rightarrow\left\{X_{1}, X_{2}, \ldots, X_{r}\right\}$ una $r$-coloración libre de $Q$ de $V(D)$ y sea

$$
F=\left\{u \rightarrow v \text { en } A(D): u \in \varphi^{-1}\left(X_{i}\right), v \in \varphi^{-1}\left(X_{j}\right), i \neq j\right\},
$$

entonces $F$ es libre de $Q$ y $r=\omega(D \backslash F)$ es máxima.

El siguiente teorema aparece sin demostración en [NL99] como generalización del teorema $1.1^{2}$.

Teorema 1.2 (Teorema 6.2 en [NL99]). Sean D una digráfica y $\alpha=\left\{\alpha_{i}\right\}_{i \in V(D)}$ una familia no vacía de digráficas, si $Q$ es adecuada entonces

$$
\vec{\omega}(D[\alpha], Q)=\operatorname{máx}_{W \in T r_{Q}(D)}\left\{\omega\left(W^{\circ}\right)+\sum_{j \in J} \vec{\omega}\left(\alpha_{j}, Q\right)\right\},
$$

donde $J=V(D) \backslash V\left(W^{\circ}\right)$.

\footnotetext{
${ }^{2}$ página 11
} 
Demostración. Sean $W \in \operatorname{Tr}_{Q}(D), \alpha_{j}^{\prime} \in \operatorname{Tr}_{Q}\left(\alpha_{j}\right)$ de tal manera que $\vec{\omega}(D, Q)=\omega(W)$ y $\vec{\omega}\left(\alpha_{j}, Q\right)=\omega\left(\alpha_{j}^{\prime}\right)$ para toda $j \in V(D)$. Sea $\alpha^{\prime}=\left\{\alpha_{j}^{\prime}\right\}_{j \in V(D)}$ y notemos que

$$
D[\alpha] \backslash A\left(W\left[\alpha^{\prime}\right]\right)=D \backslash A(W)\left[\left\{\alpha_{j} \backslash A\left(\alpha_{j}^{\prime}\right)\right\}_{j \in V(D)}\right]
$$

y dado que $D \backslash A(W)$ y $\alpha_{j} \backslash A\left(\alpha_{j}^{\prime}\right)$ son libres de $Q$, entonces $D[\alpha] \backslash A\left(W\left[\alpha^{\prime}\right]\right)$ también es libre de $Q$. Lo anterior sucede si y solo si $W\left[\alpha^{\prime}\right] \in \operatorname{Tr}_{Q}(D[\alpha])$ y es directo ver que

$$
\omega\left(W\left[\alpha^{\prime}\right]\right)=\omega\left(W^{\circ}\right)+\sum_{j \in J} \omega\left(\alpha_{j}^{\prime}\right)
$$

por lo tanto

$$
\vec{\omega}(D[\alpha], Q) \geq \operatorname{máx}_{W \in \operatorname{Tr}_{Q}(D)}\left\{\omega\left(W^{\circ}\right)+\sum_{j \in J} \vec{\omega}\left(\alpha_{j}, Q\right)\right\} .
$$

Para demostrar la otra desigualdad, sea

$$
\varphi: V(D[\alpha]) \rightarrow\left\{X_{1}, X_{2}, \ldots, X_{\vec{\omega}(D[\alpha])}\right\}
$$

una $\vec{\omega}(D[\alpha], Q)$-coloración óptima libre de $Q$. Si para alguna $u \in V(D)$, se tiene que $\alpha_{u}$ no es monocromático y un color $X_{k}$ que aparece en $\alpha_{u}$, aparece también en alguna $\alpha_{w}$ con $u \neq w$, borremos los vértices de color $X_{k}$ en $\alpha_{u}$ conservando sin alteración a $\alpha_{w}$. Repitamos dicho procedimiento hasta no obtener reducción posible y dado que ningún color y ninguna $\alpha_{u}$ desaparece en el proceso, obtenemos una familia no vacía de digráficas $\alpha^{\prime}=\left\{\alpha_{u}^{\prime}\right\}_{u \in V(D)}$ tal que $\alpha_{u}^{\prime} \subseteq \alpha_{u}$ para toda $u \in V(D)$ y satisface que la restricción de $\varphi$ en $D\left[\alpha^{\prime}\right]$ es una $\vec{\omega}(D[\alpha], Q)$-coloración.

Sea $\Gamma$ una $Q$-subdigráfica en $D$. Para toda $w \in V(\Gamma)$, sea un vértice $v_{w} \in \alpha_{w}^{\prime} \mathrm{y}$ claramente existe una $Q$-subdigráfica $\hat{\Gamma}$ en $D\left[\alpha^{\prime}\right]$ tal que

$$
V(\hat{\Gamma})=\left\{v_{w}: w \in V(\Gamma)\right\}
$$

y $\pi(\hat{\Gamma})=\Gamma$, donde $\pi$ es la proyección natural $\pi: V(D[\alpha]) \rightarrow V(D)$.

Dado que $\varphi$ no deja $Q$-subdigráficas, entonces existe una flecha $v_{u(\Gamma)} \rightarrow v_{w(\Gamma)}$ en $A(\hat{\Gamma})$-por lo tanto una flecha $u(\Gamma) \rightarrow w(\Gamma)$ en $A(\Gamma)-$, tal que $\varphi\left(v_{u(\Gamma)}\right)=\varphi\left(v_{w(\Gamma)}\right)$. Como el proceso de reducción ha terminado, se sigue que $\alpha_{u(\Gamma)}^{\prime}$ y $\alpha_{w(\Gamma)}^{\prime}$ son monocromáticos.

Sea $W$ una subdigráfica generadora de $D$ con

$$
A(W)=\left\{u \rightarrow z \text { en } A(D): \varphi\left(\alpha_{u}^{\prime}\right)=\varphi\left(\alpha_{z}^{\prime}\right)\right\}
$$

entonces $D \backslash A(W)$ es libre de $Q$, por lo tanto $W \in \operatorname{Tr}_{Q}(D)$. Obtenemos que para toda componente no trivial $S$ de $W, S\left[\left\{\alpha_{u}^{\prime}\right\}_{u \in V(S)}\right]$ es monocromática.

Por otro lado, el número de colores que aparecen en $\alpha_{u}^{\prime}$ es al menos $\vec{\omega}\left(\alpha_{u}, Q\right)$ para todo vértice aislado $u$ de $W$. Se sigue que

$$
\vec{\omega}(D[\alpha], Q) \leq \omega\left(W^{\circ}\right)+\sum_{j \in J} \vec{\omega}\left(\alpha_{j}, Q\right),
$$


por lo que

$$
\vec{\omega}(D[\alpha], Q) \leq \operatorname{máx}_{W \in \operatorname{Tr}_{Q}(D)}\left\{\omega\left(W^{\circ}\right)+\sum_{j \in J} \vec{\omega}\left(\alpha_{j}, Q\right)\right\} .
$$

Con lo anterior se obtiene la igualdad deseada.

La siguiente definición es una generalización de la definición $1.9^{3}$ sobre coloraciones $\vec{\omega}$-agudas e $\overrightarrow{\omega_{3}}$-agudas.

Definición 1.11. Una r-coloración se dirá $Q$-óptima si realiza la inconexión libre de Q. Una digráfica $D$ se dice $Q_{-}^{-a g u d a}$ si

(i) existe una r-coloración de $V(D) Q$-óptima,

(ii) dicha coloración tiene exactamente una clase cromática singular y

(iii) no existe otra r-coloración que fija más de una clase cromática singular.

La siguiente proposición aparece en [NL99] sin demostración como generalización de la proposición $1.4^{4}$.

Proposición 1.6 (Proposición 6.4 en [NL99]). Supongamos que $Q$ es adecuada.

(i) Si $D$ es una digráfica $Q$-aguda y $B$ una digráfica, entonces

$$
\vec{\omega}(D[B], Q)=\vec{\omega}(D, Q)+\vec{\omega}(B, Q)-1 .
$$

(ii) Si $D$ y $B$ son $Q$-agudas, entonces $D[B]$ es también $Q$-aguda.

(iii) Si T es un torneo circulante $Q$-agudo y $\alpha=\left\{\alpha_{i}\right\}_{i \in V(T)}$ es una familia no vacía de digráficas, entonces

$$
\vec{w}(T[\alpha], Q)=\vec{w}(T, Q)+\operatorname{máx}_{i \in V(T)}\left\{\vec{w}\left(\alpha_{i}, Q\right)\right\}-1 .
$$

Demostración. (i) Sea $W \in \operatorname{Tr}_{Q}(D)$. Como $D$ es $Q$-aguda entonces consideremos una partición con el vértice singular $u$, por lo que $J=V(D) \backslash V\left(W^{\circ}\right)=\{u\}$, así $W^{\circ}=V(D) \backslash\{u\}$ y $\omega\left(W^{\circ}\right)=\vec{\omega}(D, Q)-1$.

Por otra parte como $D[B]=D[\alpha]$ y toda $\alpha_{j} \cong B$, entonces por el teorema 1.2 tenemos que

$$
\begin{aligned}
\vec{\omega}(D[B], Q) & =\operatorname{máx}_{W \in \operatorname{Tr}_{Q}(D)}\left\{\omega\left(W^{\circ}\right)+\sum_{j \in J} \vec{\omega}\left(\alpha_{j}, Q\right)\right\} \\
& =\operatorname{máx}_{W \in \operatorname{Tr}_{Q}(D)}\{\omega(D, Q)-1+\vec{\omega}(B, Q)\} \\
& =\vec{\omega}(D, Q)+\vec{\omega}(B, Q)-1 .
\end{aligned}
$$

\footnotetext{
${ }^{3}$ página 12

${ }^{4}$ página 12
} 
(ii) Supongamos que existen al menos dos vértices singulares $u, v \in V(D[B])$ en copias distintas de $B$. Sea $\pi$ la proyección natural de $D[B]$ sobre $D$ definida por $\pi: V(D[B]) \rightarrow V(D)$ y denotemos por $B_{u}$ y $B_{v}$ a la copias isomorfas de $B$ tales que $\pi\left(B_{u}\right)=u$ y $\pi\left(B_{v}\right)=v$ respectivamente. Sea

$$
\varphi: V(D[B]) \rightarrow\left\{X_{1}, X_{2}, \ldots, X_{r}\right\}
$$

una $r$-coloración $Q$-óptima, con $r \geq 3$ y supongamos sin pérdida de generalidad que $\varphi(u)=X_{1}$ y $\varphi(v)=X_{2}$.

Notemos que ni $u$ ni $v$ están en $Q$-subdigráfica alguna, entonces para todo vértice $w \in V\left(B_{u}\right)$ (resp. $w \in V\left(B_{v}\right)$ ), se tiene que $w$ tampoco está en $Q$-subdigáfica alguna, por lo que podemos asignar los colores $\varphi\left(B_{u}\right)=X_{1}$ y $\varphi\left(B_{v}\right)=X_{2}$ sin inducir $Q$-subdigráficas bien coloreadas. Así $u$ y $v$ serían vértices singulares en $\pi(D[B])=D$, que es una contradicción al hecho de que $D$ es $Q$-agudo.

La observación $1.3^{5}$ sobre coloraciones heredadas de una composición, es válida para digráficas $Q$-agudas y nos proporciona una coloración con un vértice singular, por lo que $D[B]$ es $Q$-aguda.

(iii) De manera similar a (i), si $W^{\circ}=V(D) \backslash\{u\}$, entonces

$$
J=V(D) \backslash V\left(W^{\circ}\right)=\{u\} \quad \text { y } \quad \omega\left(W^{\circ}\right)=\vec{\omega}(D, Q)-1 .
$$

Además notemos que

$$
\operatorname{máx}_{W \in \operatorname{Tr}_{Q}(D)}\left\{\omega\left(W^{\circ}\right)+\vec{\omega}\left(\alpha_{i}, Q\right)\right\}=\omega\left(W^{\circ}\right)+\operatorname{máx}\left\{\vec{\omega}\left(\alpha_{i}, Q\right): i \in V(T)\right\},
$$

por lo tanto

$$
\begin{aligned}
\vec{\omega}(T[\alpha], Q) & =\omega\left(W^{\circ}\right)+\operatorname{máx}_{i \in V(T)}\left\{\vec{\omega}\left(\alpha_{i}, Q\right)\right\} \\
& =\vec{\omega}(T, Q)+\operatorname{máx}_{i \in V(T)}\left\{\vec{\omega}\left(\alpha_{i}, Q\right)\right\}-1 .
\end{aligned}
$$

El siguiente resultado proporciona una herramienta para simplificar el cálculo de la inconexión acíclica de torneos, el cual afirma que no es necesario analizar todos los ciclos contenidos en el torneo, basta solo considerar sus triángulos y cuadrados dirigidos.

Teorema 1.3 (Proposición 6.3 en [NL99]). Para todo torneo T, se tiene que

$$
\vec{\omega}(T)=\vec{\omega}\left(T,\left\{\vec{C}_{3}, \vec{C}_{4}\right\}\right)
$$

\footnotetext{
${ }^{5}$ página 13
} 
Demostración. Como $\vec{\omega}\left(T, \overrightarrow{C_{3}}\right)=\overrightarrow{\omega_{3}}(T)$, entonces la desigualdad

$$
\vec{\omega}(T) \leq \vec{\omega}\left(T,\left\{\overrightarrow{C_{3}}, \overrightarrow{C_{4}}\right\}\right) \leq \overrightarrow{\omega_{3}}(T)
$$

es clara.

Mostremos ahora que $\vec{\omega}\left(T,\left\{\overrightarrow{C_{3}}, \overrightarrow{C_{4}}\right\}\right) \leq \vec{\omega}(T)$. Sea

$$
\varphi: V(T) \rightarrow\left\{X_{1}, X_{2}, \ldots, X_{\vec{\omega}^{+}(T)}\right\}
$$

una $\vec{\omega}^{+}(T)$-coloración de $V(T)$, donde $\vec{\omega}^{+}(T) \geq 3$. Entonces existe al menos un ciclo dirigido bien coloreado

$$
\vec{C}_{r}: u_{0} \rightarrow u_{1} \rightarrow \cdots \rightarrow u_{r-1} \rightarrow u_{0}
$$

Supongamos que este ciclo es de longitud mínima y que $r \geq 5$. Sin pérdida de generalidad supongamos que $\varphi\left(u_{0}\right)=X_{1}$ y que $\varphi\left(u_{1}\right)=X_{2}$. Tenemos los siguientes casos:

(i) $\varphi\left(u_{2}\right)=X_{1}$. Observe que $\varphi\left(u_{3}\right) \neq \varphi\left(u_{0}\right)$.

- Si $u_{0} \rightarrow u_{3}$, entonces

$$
\vec{C}_{r-2}: u_{0} \rightarrow u_{3} \rightarrow \cdots \rightarrow u_{r-1} \rightarrow u_{0}
$$

está bien coloreado, en contradicción con la selección de $\overrightarrow{C_{r}}$ de longitud mínima.

- Si $u_{3} \rightarrow u_{0}$, entonces

$$
\vec{C}_{4}: u_{0} \rightarrow u_{1} \rightarrow u_{2} \rightarrow u_{3} \rightarrow u_{0}
$$

está bien coloreado, que es una contradicción similar al caso anterior.

(ii) $\varphi\left(u_{2}\right) \neq X_{1}$. Sin pérdida de generalidad supongamos que $\varphi\left(u_{2}\right)=X_{3}$, entonces:

- Si $u_{0} \rightarrow u_{2}$, entonces

$$
\vec{C}_{r-1}: u_{0} \rightarrow u_{2} \rightarrow \cdots \rightarrow u_{r-1} \rightarrow u_{0}
$$

está bien coloreado, que es una contradicción similar al inciso (i).

- Si $u_{2} \rightarrow u_{0}$, entonces

$$
\vec{C}_{3}: u_{0} \rightarrow u_{1} \rightarrow u_{2} \rightarrow u_{0}
$$

es heterocromático, que es imposible.

Así, estos ciclos son de longitud menor a $r$, lo que contradice el hecho de que $\vec{C}_{r}$ es de longitud mínima. 
En todos los casos se obtiene un ciclo de longitud menor a $r \geq 5$, por lo que concluimos que $\vec{\omega}\left(T,\left\{\overrightarrow{C_{3}}, \overrightarrow{C_{4}}\right\}\right) \leq \vec{\omega}(T)$.

Corolario 1.4. Para todo torneo $T$, la inconexión acíclica $\vec{\omega}(T)$ es el máximo número de colores, de una coloración de $V(T)$ que no produce $\left\{\overrightarrow{C_{3}}, \overrightarrow{C_{4}}\right\}$ bien coloreados.

Demostración. Es consecuencia directa del teorema 1.3 y la proposición $1.1^{6}$.

Observación 1.4. Dado un torneo T, para probar que $\vec{\omega}(T)=r$ (resp. $\vec{\omega}_{3}(T)=r$ ), es suficiente con probar que $\vec{\omega}(T) \leq r$ (resp. $\overrightarrow{\omega_{3}}(T) \leq r$ ) y exhibir una $r$-coloración libre de $\left\{\overrightarrow{C_{3}}, \overrightarrow{C_{4}}\right\}$ bien coloreados (resp. libre de $\overrightarrow{C_{3}}$ heterocromáticos), es decir óptima, de tal manera que $\vec{\omega}(T)$ (resp. $\overrightarrow{\omega_{3}}(T)$ ) se alcanza exactamente en $r$.

\footnotetext{
${ }^{6}$ página 4
} 


\section{La diferencia $\overrightarrow{\omega_{3}}(T)-\vec{\omega}(T)$}

Recordemos que dado un torneo $T$, se cumple que $\vec{\omega}(T) \leq \overrightarrow{\omega_{3}}(T)$. El principal objetivo del presente capítulo es el de responder a la pregunta:

¿Qué tan grande puede ser la diferencia $\overrightarrow{\omega_{3}}(T)-\vec{\omega}(T)$ ?

En este capítulo proponemos una familia infinita de torneos, que son generalizaciones del ejemplo propuesto por Neumann-Lara en [NL99], para los cuales $\vec{\omega}(T)<\overrightarrow{\omega_{3}}(T)$. Más aún, respondemos afirmativamente al siguiente:

Problema 2.1 (Problema 6.6 en [NL99]). ¿Existe algún torneo T para el cual $\vec{\omega}(T)=2$ $y \overrightarrow{\omega_{3}}(T)$ es arbitrariamente grande?

Así mismo, el principal resultado de este capítulo es una generalización del problema anterior, en el que respondemos afirmativamente a la pregunta:

Problema 2.2. ¿Existe algún torneo $T$ para el cual $\vec{\omega}(T)=r$ y $\overrightarrow{\omega_{3}}(T)=s$, donde $r, s$ son enteros positivos arbitrarios tales que $2 \leq r \leq s$ ?

Los resultados anteriores se encuentran publicados en [CALl13].

\section{1. $\quad$ El epimorfismo $\pi$ y la digráfica auxiliar $D_{n+1}$}

En esta sección exhibimos algunos resultados auxiliares, que nos serán de gran ayuda para definir una familia infinita de torneos que responden a los problemas anteriores.

En lo sucesivo denotaremos simplemente por $\mathbf{i}$ al conjunto $\{(i, 1),(i, 2)\}$ con $i \in \mathbb{N}$. Sean

$$
\left.T_{2 n+1}=(\{1,2, \ldots, n\} \times\{1,2\}) \cup\{0\}, A\left(T_{2 n+1}\right)\right)
$$

el torneo de orden $2 n+1 \mathrm{y}$

$$
D_{n+1}=\left(\{0,1,2, \ldots, n\}, A\left(D_{n+1}\right)\right)
$$

la digráfica de orden $n+1$, asociados de la siguiente forma: sea $\pi: V\left(T_{2 n+1}\right) \rightarrow V\left(D_{n+1}\right)$ el epimorfismo reflexivo, definido por

$$
\pi(v)= \begin{cases}0, & \text { si } v=\{0\} \\ i, & \text { si } v=(i, j)\end{cases}
$$

tal que cumple las siguientes propiedades sobre los conjuntos de flechas $A\left(T_{2 n+1}\right)$ y $A\left(D_{n+1}\right)$. 
(a) La flecha $(i, 1) \rightarrow(i, 2)$ está en $A\left(T_{2 n+1}\right)$.

(b) Si $D_{n+1}\langle\{i, j\}\rangle$ es la flecha simétrica $i \leftrightarrow j$, entonces el ciclo de longitud cuatro

$$
\overrightarrow{C_{4}}:(i, 1) \rightarrow(j, 1) \rightarrow(i, 2) \rightarrow(j, 2) \rightarrow(i, 1)
$$

es una subdigráfica de $T_{2 n+1}$.

(c) Si $D_{n+1}\langle\{i, j\}\rangle$ es la flecha $i \rightarrow j$, entonces $\mathbf{i} \Rightarrow \mathbf{j}$ en $T_{2 n+1}$.

(d) Si $0 \rightarrow i$ y $0 \leftarrow j$ son flechas en $A\left(D_{n+1}\right)$, entonces $0 \Rightarrow \mathbf{i}$ y $0 \Leftarrow \mathbf{j}$ respectivamente en $T_{2 n+1}$.

Si se cumplen (a) y (b), entonces $T\langle\{\mathbf{i}, \mathbf{j}\}\rangle \cong S R_{4}$ y de manera similar, si se cumplen (a) y (c), entonces $T\langle\{\mathbf{i}, \mathbf{j}\}\rangle \cong T T_{4}$. En otras palabras, si tenemos una flecha simétrica en $D_{n+1} \backslash\{0\}$, entonces es la imagen de un torneo semirregular y si es una flecha simple, entonces es la imagen de un torneo transitivo, ambos de orden cuatro que son únicos salvo isomorfismos. En la figura 2.1 podemos observar la acción inversa de $\pi$ de la digráfica $D_{n+1}$ al torneo $T_{2 n+1}$.

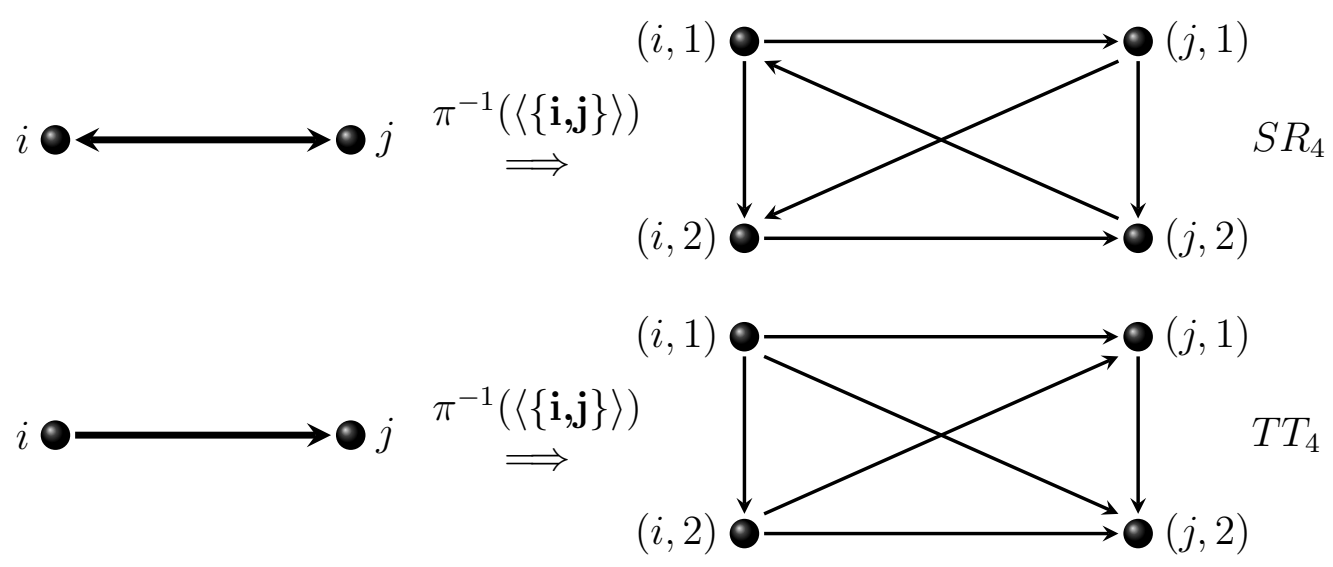

Figura 2.1: El epimorfismo $\pi(\{\mathbf{i}, \mathbf{j}\})=\{i, j\}, T\langle\{\mathbf{i}, \mathbf{j}\}\rangle \cong S R_{4}$ y $T\langle\{\mathbf{i}, \mathbf{j}\}\rangle \cong T T_{4}$.

Daremos por entendido que si estamos hablando de $i$ en el contexto de la digráfica $D_{n+1}$, entonces es el vértice resultante de haber aplicado el epimorfismo reflexivo descrito en (2.1) al conjunto $\mathbf{i}=\{(i, 1),(i, 2)\}$. Si hablamos de $\mathbf{i}$ en el contexto de algún torneo $T_{2 n+1}$, entonces será el conjunto antes dicho. De manera similar, definimos el conjunto

$$
\mathbf{i}+\mathbf{j}=\{(i+j, 1),(i+j, 2)\} .
$$

El epimorfismo reflexivo $\pi$ juega un papel importante para simplificar la definición de la familia infinita de torneos $\mathfrak{V}_{n}$ más adelante.

Proposición 2.1. $\vec{\omega}\left(S R_{4}\right)=\overrightarrow{\omega_{3}}\left(S R_{4}\right)=3$. 
Demostración. Sea $S R_{4}$ el torneo semiregular de orden cuatro como en la figura 2.1. Es claro que la única 3-coloración óptima $\varphi: V\left(S R_{4}\right) \rightarrow\left\{X_{1}, X_{2}, X_{3}\right\}$ es

$$
\varphi(u)= \begin{cases}X_{1}, & \text { si } u \in\{(i, 1),(j, 2)\} \\ X_{2}, & \text { si } u=(i, 2) \\ X_{3}, & \text { si } u=(j, 1)\end{cases}
$$

y es imposible agregar un cuarto color sin que se formen triángulos o cuadrados bien coloreados.

Para toda $m \geq 1$, construyamos la digráfica auxiliar $D_{2 m+1}=\left(V\left(D_{2 m+1}\right), A\left(D_{2 m+1}\right)\right)$ inductivamente como sigue:

$$
\begin{aligned}
D_{1} & =\{0\} \\
D_{3} & =\overrightarrow{C_{3}}: 0 \rightarrow 1 \rightarrow 2 \rightarrow 0 \\
& \vdots \\
D_{2 m+1} & =\left(V\left(D_{2 m+1}\right), A\left(D_{2 m+1}\right)\right),(m \geq 2),
\end{aligned}
$$

donde

$$
\begin{aligned}
V\left(D_{2 m+1}\right)= & V\left(D_{2 m-1}\right) \cup\{2 m-1,2 m\} \\
A\left(D_{2 m+1}\right)= & A\left(D_{2 m-1}\right) \cup\left\{i \rightarrow(2 m-1),(2 m) \rightarrow i: i \in \mathbb{Z}_{2 m-2}\right\} \cup \\
& \{(2 m-1) \rightarrow(2 m),(2 m-1) \rightarrow(2 m-3),(2 m-2) \rightarrow(2 m)\} .
\end{aligned}
$$

En la figura 2.2 mostramos la construcción recursiva de las digráficas auxiliares de los primeros tres miembros de la familia $\mathfrak{V}_{n}$ y en la figura 2.3 mostramos la construcción en el caso general de la digráfica auxiliar $D_{2 m+1}$.

\section{2. $\quad$ El torneo $T_{9}$ y la familia $\mathfrak{V}_{n}$}

El torneo $T$ de nueve vértices del ejemplo 4.2 expuesto en [NL99], nos muestra que la desigualdad estricta $\vec{\omega}(T)<\overrightarrow{\omega_{3}}(T)$ es posible en torneos, por lo que inspirados en dicho ejemplo, definimos la familia $\mathfrak{V}_{n}$ como una generalización de dicho ejemplo.

La familia de torneos $\mathfrak{V}_{n}=\left\{T_{8 n+1}: n \in \mathbb{N}\right\}$ se define como el conjunto de torneos $T_{8 n+1}$ tales que

$$
\begin{aligned}
& V\left(T_{8 n+1}\right)=(\{1,2, \ldots, 4 n\} \times\{1,2\}) \cup\{0\} \quad \mathrm{y} \\
& A\left(T_{8 n+1}\right)=\left\{\pi^{-1}\left(D_{4 n+1}\langle\{i, j\}\rangle\right): i, j \in V\left(D_{4 n+1}\right)\right\},
\end{aligned}
$$

donde $\pi: V\left(T_{8 n+1}\right) \rightarrow V\left(D_{4 n+1}\right)$ es el epimorfismo reflexivo definido en (2.1).

La figura 2.4 muestra un ejemplo de la digráfica auxiliar $D_{13}$ asociada al torneo $T_{25}$. 

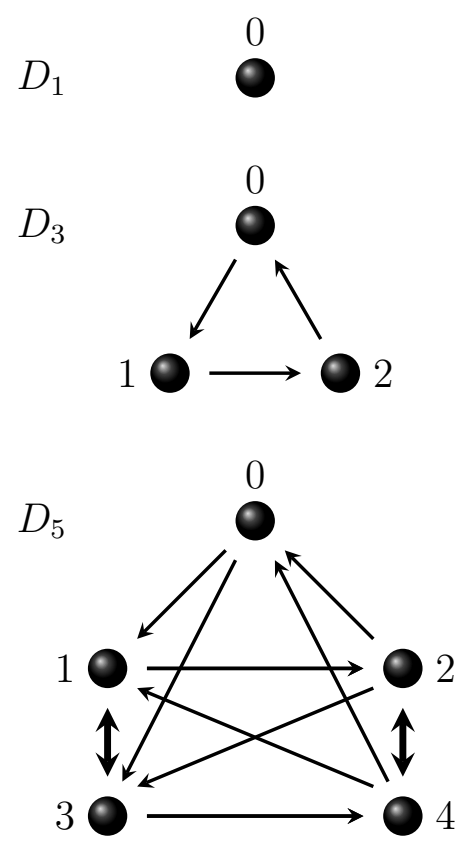

Figura 2.2: Las digráficas auxiliares de la familia $\mathfrak{V}_{n}$.

Observación 2.1. El torneo $T$ de nueve vértices del ejemplo 4.2 de [NL99] coincide con el torneo $T_{9} \in \mathfrak{V}_{n}$, cuya digráfica auxiliar se muestra en la figura 2.2 y el torneo se muestra en la figura 2.5. Este torneo satisface que $\vec{\omega}\left(T_{9}\right)=2$ y $\vec{\omega}_{3}\left(T_{9}\right)=3$, el cual es el único ejemplo conocido en la literatura de un torneo $T$ que cumple que $\vec{\omega}(T)<\overrightarrow{\omega_{3}}(T)$. Notemos además que dada $n \in \mathbb{N}$,

$$
T_{8 n+1}\langle\{0, \mathscr{2 i}-3,2 i-\mathscr{2}, \mathscr{2 i}-\mathbf{1}, \mathscr{2} i\}\rangle \cong T_{9} \in \mathfrak{V}_{n}
$$

para toda $2 \leq i \leq 2 n$. Una posible 3-coloración de $V\left(T_{9}\right)$ es

$$
\varphi(v)= \begin{cases}X_{1}, & \text { si } v=\{0\}, \\ X_{2}, & \text { si } v \in\{\mathbf{1}, \mathscr{2}\}, \\ X_{3}, & \text { si } v \in\{3,4\}\end{cases}
$$

Esta coloración es libre de $\vec{C}_{3}$ heterocromáticos, sin embargo no es libre de $\vec{C}_{4}$ bien coloreados, ya que

$$
\vec{C}_{4}:(1,1) \rightarrow(3,1) \rightarrow(1,2) \rightarrow(3,2) \rightarrow(1,1)
$$

y

$$
\vec{C}_{4}:(2,1) \rightarrow(4,1) \rightarrow(2,2) \rightarrow(4,2) \rightarrow(2,1)
$$

son cuadrados bien coloreados. 


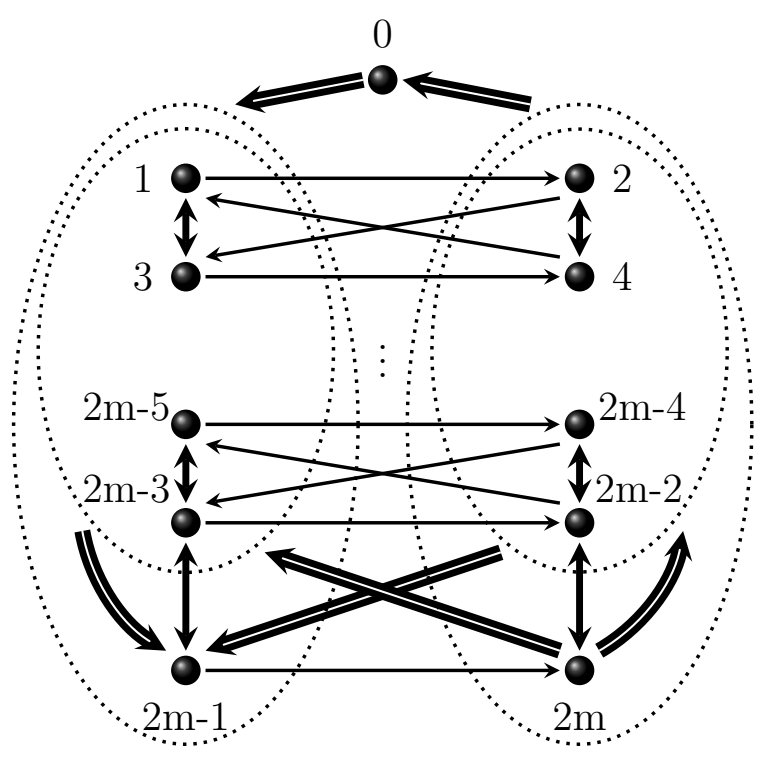

Figura 2.3: La digráfica auxiliar $D_{2 m+1}$.

Lema 2.1. La coloración definida en (2.2) para $T_{9} \in \mathfrak{V}_{n}$ es la única 3-coloración óptima que realiza $\vec{\omega}_{3}$, en el sentido que los conjunto de vértices $V(T\langle\{\mathbf{1}, 2\}\rangle)$ y $V(T\langle\{3,4\}\rangle)$ son monocromáticos.

Demostración. Sea $\psi: V\left(T_{9}\right) \rightarrow\left\{X_{1}, X_{2}, X_{3}\right\}$ una 3-coloración óptima. Supongamos por contradicción que $|\psi(\{\mathbf{1}, \mathbf{2}\})| \geq 2$. Tenemos los siguientes casos para la cardinalidad de la coloración $\psi$.

Caso 1. Si $|\psi(\{\mathbf{1}, \mathbf{2}\})|=2$, supongamos sin pérdida de generalidad que se tiene que $\psi(\{\mathbf{1}, \mathbf{2}\})=\left\{X_{1}, X_{2}\right\}$. Tenemos los siguientes subcasos.

(i) Si $1 \subseteq X_{1}$ y $2 \subseteq X_{2}$, tenemos que $X_{3}=\emptyset$. En caso contrario, para todo vértice $w \in\{0\} \cup \mathbf{3} \cup \mathbf{4}$, existen los vértices $u \in \mathbf{1}$ y $v \in \mathbf{2}$ tales que $\overrightarrow{C_{3}}: u \rightarrow v \rightarrow w \rightarrow u$ es heterocromático, lo cual es imposible.

(ii) Si $1 \cap X_{1} \neq \emptyset$, entonces $1 \cap X_{2} \neq \emptyset$ y $2 \subseteq X_{2}$. Sin pérdida de generalidad supongamos que $(1,1) \in X_{1}$ y $(1,2) \in X_{2}$. Los casos $(1,1) \in X_{2}$ y $(1,2) \in X_{1}$ son análogos.

1. Observe que $(\{0\} \cup 4) \cap X_{3}=\emptyset$, ya que para toda $u \in\{0\} \cup \mathbf{4}$ existe $v \in \mathbf{2}$ tal que $\overrightarrow{C_{3}}:(1, i) \rightarrow v \rightarrow u \rightarrow(1, i)$ es heterocromático para $i \in\{1,2\}$.

2. Si $(3,2) \in X_{3}$ entonces $\overrightarrow{C_{3}}:(1,1) \rightarrow(1,2) \rightarrow(3,2) \rightarrow(1,1)$ es heterocromático. Por lo tanto $(3,2) \notin X_{3}$. 


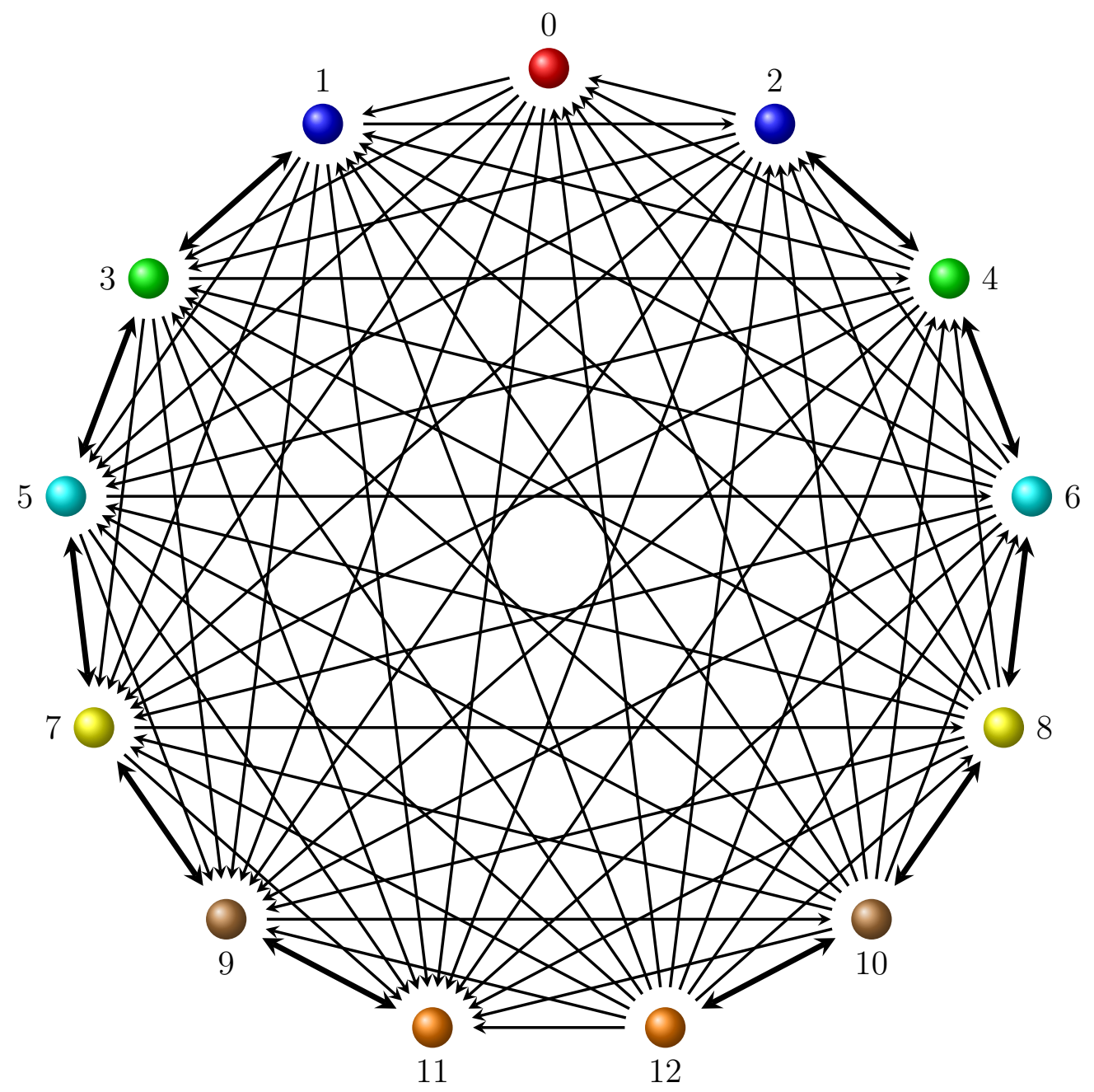

Figura 2.4: Digráfica $D_{13}$ asociada al torneo $T_{25} \in \mathfrak{V}_{n}$.

3. Notemos que

$$
\begin{aligned}
& \Delta_{1}: \quad(2,1) \rightarrow(3,1) \rightarrow(4,2) \rightarrow(2,1) \quad \text { y } \\
& \Delta_{2}: \quad(2,2) \rightarrow(3,1) \rightarrow(4,1) \rightarrow(2,2)
\end{aligned}
$$

son $\overrightarrow{C_{3}}$ dirigidos en $T_{9}$. Si $(3,1) \in X_{3}$ y $4 \cap X_{1} \neq \emptyset$, entonces $\Delta_{1}$ о $\Delta_{2}$ es heterocromático, ya que $\mathbf{2} \subseteq S_{2}$. Entonces tenemos que $\mathbf{4} \subseteq S_{2}$ (por el inciso $\left.1,4 \cap X_{3}=\emptyset\right)$ y $(3,1) \notin X_{3}$.

Así, obtenemos que $X_{3}=\emptyset$, que es imposible.

(iii) Si $\mathbf{1} \subseteq X_{1}, \mathbf{2} \cap X_{1} \neq \emptyset$ y $\mathbf{2} \cap X_{2} \neq \emptyset$, observemos que si volteamos todas las flechas del torneo $T_{9}$, entonces este subcaso es simétrico a (ii).

(iv) Si $\mathbf{1} \cap X_{i} \neq \emptyset$ y $\mathbf{2} \cap X_{i} \neq \emptyset$ para $i \in\{1,2\}$, supongamos sin pérdida de generalidad que $(1,1) \in X_{1}$ y $(1,2) \in X_{2}$. 


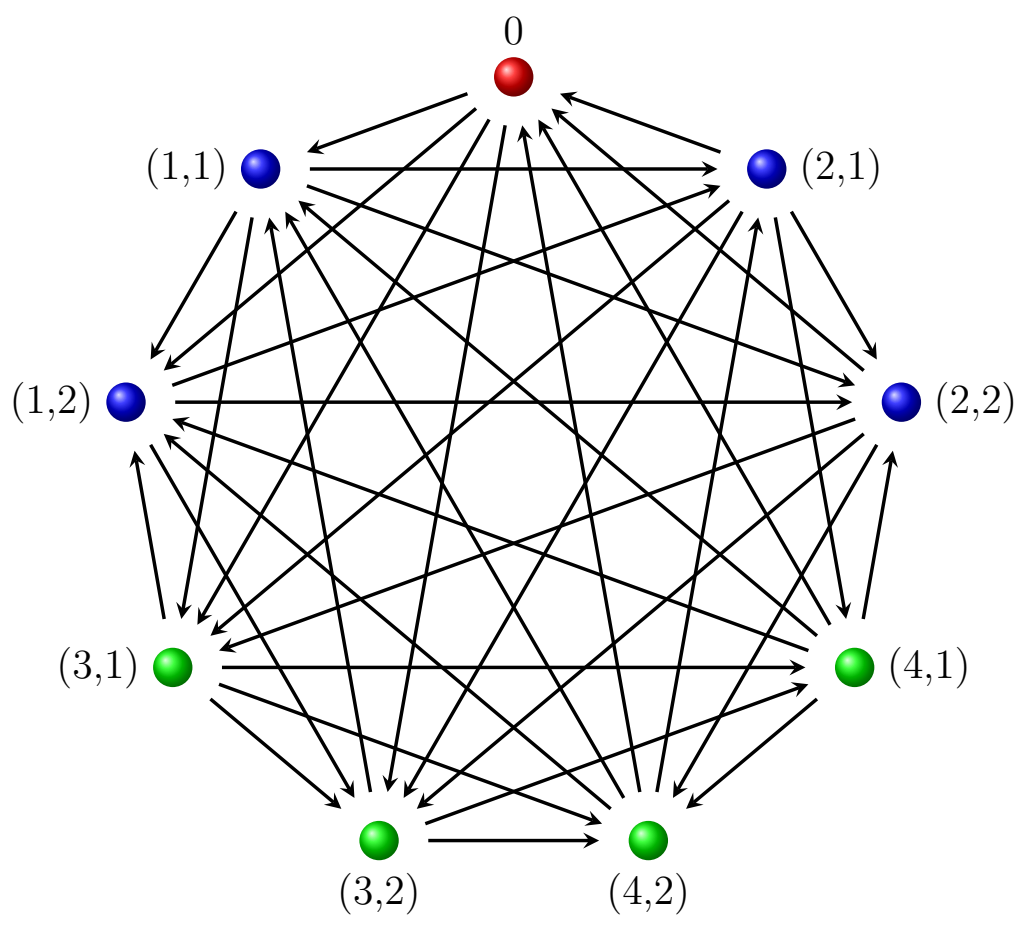

Figura 2.5: El torneo $T_{9} \in \mathfrak{V}_{n}$.

1. Note que para toda $i, j \in\{1,2\}$, existe $\overrightarrow{C_{3}}: 0 \rightarrow(1, i) \rightarrow(2, j) \rightarrow 0$.

2. Supongamos que $(2,1) \in X_{1}$ y $(2,2) \in X_{2}$. Si $0 \in X_{3}$ entonces

$$
\overrightarrow{C_{3}}: 0 \rightarrow(1, i) \rightarrow(2, j) \rightarrow 0
$$

es heterocromático para $i, j \in\{1,2\}$ tal que $i \neq j$. Así $0 \notin X_{3}$.

3. Si $(k, 2) \in X_{3}$ para $k \in\{3,4\}$, entonces

$$
\overrightarrow{C_{3}}:(1,1) \rightarrow(2,2) \rightarrow(k, 2) \rightarrow(1,1)
$$

es heterocromático. Por lo tanto $(k, 2) \notin X_{3}$ para $k \in\{3,4\}$.

4. Si $(k, 1) \in X_{3}$ para $k \in\{3,4\}$, entonces

$$
\overrightarrow{C_{3}}:(1,2) \rightarrow(2,1) \rightarrow(k, 1) \rightarrow(1,2)
$$

es heterocromático. Así $(k, 1) \notin X_{3}$ para $k \in\{3,4\}$.

5. Por los incisos 1,2 y 3 , tenemos que $X_{3}=\emptyset$, lo cual es imposible.

Los casos $(2,1) \in X_{2}$ y $(2,2) \in X_{1}$ se demuestran de manera similar.

Caso 2. Si $|\psi(\{\mathbf{1}, \mathbf{2}\})|=3$, tenemos los siguientes subcasos:

(i) Si $\mathbf{1} \subseteq X_{1}, \mathbf{2} \cap X_{2} \neq \emptyset$ y $\mathbf{2} \cap X_{3} \neq \emptyset$. Sin pérdida de generalidad, supongamos que $(2,1) \in X_{2}$ y $(2,2) \in X_{3}$. 
1. Si $\mathbf{3} \cap X_{2} \neq \emptyset$ o $\mathbf{3} \cap X_{3} \neq \emptyset$, entonces existe $u \in \mathbf{1}$ tal que

$$
\overrightarrow{C_{3}}: u \rightarrow(2,2) \rightarrow v \rightarrow u \quad \text { о } \quad \overrightarrow{C_{3}}: u \rightarrow(2,1) \rightarrow v \rightarrow u
$$

es heterocromático, para alguna $v \in \mathbf{3}$. Así $\mathbf{3} \subseteq X_{1}$.

2. Si $(4,2) \in X_{1}$, entonces $\overrightarrow{C_{3}}:(2,1) \rightarrow(2,2) \rightarrow(4,2) \rightarrow(2,1)$ es heterocromático. Por lo que $(4,2) \notin X_{1}$.

3. Si $(4,2) \in X_{2}$, entonces $\vec{C}_{3}:(1,1) \rightarrow(2,2) \rightarrow(4,2) \rightarrow(1,1)$ es heterocromático.

4. Por los incisos 2 y 3 , tenemos que $(4,2) \in X_{3}$. Si este es el caso, entonces $\overrightarrow{C_{3}}:(2,1) \rightarrow(3,1) \rightarrow(4,2) \rightarrow(2,1)$ es heterocromático.

Por los casos anteriores, tenemos una contradicción.

(ii) Si $\mathbf{1} \cap X_{1} \neq \emptyset, \mathbf{2} \cap X_{1} \neq \emptyset, \mathbf{1} \cap X_{2} \neq \emptyset$ y $\mathbf{2} \cap X_{3} \neq \emptyset$. Sean $i, j \in\{1,2\}$ tales que $i \neq j$. Sin pérdida de generalidad, supongamos que $\{(1,1),(2, i)\} \subseteq X_{1}$, $(1,2) \in X_{2}$ y $(2, j) \in X_{3}$.

1. Si $0 \in X_{1}$, entonces $\overrightarrow{C_{3}}: 0 \rightarrow(1,2) \rightarrow(2, j) \rightarrow 0$ es heterocromático.

2. Si $0 \in X_{2}$, entonces $\vec{C}_{3}: 0 \rightarrow(1,1) \rightarrow(2, j) \rightarrow 0$ es heterocromático.

3. Si $0 \in X_{3}$, entonces $\vec{C}_{3}: 0 \rightarrow(1,2) \rightarrow(2, i) \rightarrow 0$ es heterocromático.

Por los casos anteriores concluimos que $0 \notin X_{1} \cup X_{2} \cup X_{3}$, que es imposible.

(iii) Si $1 \cap X_{1} \neq \emptyset, \mathbf{1} \cap X_{2} \neq \emptyset$ y $\mathbf{2} \subseteq X_{3}$. Nótese que este subcaso es simétrico a (i) y se demuestra de manera similar.

Concluimos que el conjunto $V\left(T_{9}\langle\mathbf{1}, \mathbf{2}\rangle\right)$ es monocromático y de manera análoga, podemos demostrar que $V\left(T_{9}\langle\mathbf{3}, \mathbf{4}\rangle\right)$ es también monocromático.

El siguiente lema describe la estructura de los triángulos contenidos en los elementos de la familia $\mathfrak{V}_{n}$ y nos será de gran utilidad para probar algunas propiedades más adelante.

Lema 2.2. Un $\vec{C}_{3}: u \rightarrow v \rightarrow w \rightarrow u$ en $T_{8 n+1} \in \mathfrak{V}_{n}$ es exactamente uno de los siguientes casos:

(i) $u=0, v \in \mathscr{2 i - 1}$ y $w \in \mathscr{2} \boldsymbol{i}, 1 \leq i \leq 2 n$;

(ii) $u \in \mathscr{2 j} \mathbf{j} \cup \mathscr{2 j}, v \in \mathscr{2 i} \mathbf{1}$ y $w \in \mathscr{2 i}, 1 \leq j \leq i+2 \leq 2 n$;

(iii) si $u=(2 i-3,1)$ y $v=(2 i-1,1)$ o bien $u=(2 i-3,2)$ y $v=(2 i-1,2)$, entonces $w \in 2 \boldsymbol{i}, 2 \leq i \leq 2 n$;

(iv) si $u=(2 i, 1)$ y $v=(2 i-2,2)$ o bien $u=(2 i, 2)$ y $v=(2 i-2,1)$, entonces $w \in 2 i-1,2 \leq i \leq 2 n$; 
(v) si $u=(2 i+1,1)$ y $v=(2 i-1,2)$ o bien $u=(2 i+1,2)$ y $v=(2 i-1,1)$, entonces $w \in \mathscr{2 i}, 1 \leq i \leq 2 n-1$;

(vi) si $u=(2 i, 1), v=(2 i+2,1)$ o $u=(2 i, 2), v=(2 i+2,2)$, entonces $w \in 2 \boldsymbol{2}$ - $\mathbf{1}$, $1 \leq i \leq 2 n-1$

(vii) $u=(2 i+1,2), v=(2 i-1,1) y w \in\{(2 i-1,2),(2 i+1,1)\}, 2 \leq i \leq 2 n-1 \quad o$

(viii) $u=(2 i+2,2), v=(2 i, 1) y w \in\{(2 i, 2),(2 i+2,1)\}, 1 \leq i \leq 2 n-2$.

Más aún, toda flecha en $A\left(T_{8 n+1}\right)$ pertenece a algún triángulo dirigido.

Demostración. Se sigue directamente por la construcción del torneo $T_{8 n+1}$ de la familia $\mathfrak{V}_{n}$, para toda $n \in \mathbb{N}$. Para cada caso respectivamente tenemos lo siguiente.

(i) Observe que $0 \Rightarrow \mathbf{2 i}-\mathbf{1}, \mathbf{2} \mathbf{i}-\mathbf{1} \Rightarrow \mathbf{2 i}$ y $\mathbf{2 i} \Rightarrow 0$.

(ii) $(\mathbf{2} \mathbf{j}-\mathbf{1} \cup \mathbf{2} \mathbf{j}) \Rightarrow \mathbf{2} \mathbf{i}-\mathbf{1}, \mathbf{2} \mathbf{i}-\mathbf{1} \Rightarrow \mathbf{2} \mathbf{i}$ y $\mathbf{2} \mathbf{i} \Rightarrow(\mathbf{2} \mathbf{j}-\mathbf{1} \cup \mathbf{2} \mathbf{j})$, con $1 \leq j \leq i+2 \leq 2 n$.

(iii) Notemos que $T\langle\mathbf{2} \mathbf{j}-\mathbf{3} \cup \mathbf{2} \mathbf{j}-\mathbf{1}\rangle \cong S R_{4}$, entonces tenemos por la construcción de la familia a las flechas $(2 i-3,1) \rightarrow(2 i-1,1)$ y $(2 i-3,2) \rightarrow(2 i-1,2)$. Como $\mathbf{2} \mathbf{i}-\mathbf{1} \Rightarrow \mathbf{2} \mathbf{i}$ y $\mathbf{2} \mathbf{i} \Rightarrow \mathbf{2} \mathbf{i}-\mathbf{3}$, se tiene lo deseado para $2 \leq i \leq 2 n$.

(iv) De manera similar al caso anterior, $T\langle\mathbf{2} \mathbf{i}-\mathbf{2} \cup \mathbf{2} \mathbf{i}\rangle \cong S R_{4}$, entonces tenemos las flechas $(2 i, 1) \rightarrow(2 i-2,2)$ y $(2 i, 2) \rightarrow(2 i-2,1)$. Como $\mathbf{2} \mathbf{i}-\mathbf{1} \Rightarrow \mathbf{2 i}$ y $\mathbf{2 i - 2} \Rightarrow \mathbf{2 i - 1}$, se tiene lo deseado para $2 \leq i \leq 2 n$.

(v) Como $T\langle\mathbf{2} \mathbf{i}-\mathbf{1} \cup \mathbf{2} \mathbf{i}+\mathbf{1}\rangle \cong S R_{4}$, entonces tenemos las flechas $(2 i+1,1) \rightarrow(2 i-1,2)$ y $(2 i+1,2) \rightarrow(2 i-1,1)$. Notemos que $\mathbf{2} \mathbf{i}-\mathbf{1} \Rightarrow \mathbf{2 i}$ y $\mathbf{2} \mathbf{i} \Rightarrow \mathbf{2} \mathbf{i}+\mathbf{1}$ por lo que se tiene lo deseado para $1 \leq i \leq 2 n-1$.

(vi) Como $T\langle\mathbf{2} \mathbf{i} \cup \mathbf{2 i}+\mathbf{2}\rangle \cong S R_{4}$, entonces tenemos las flechas $(2 i, 1) \rightarrow(2 i+2,1)$ y $(2 i, 2) \rightarrow(2 i+2,2)$. Notemos que $\mathbf{2} \mathbf{i}+\mathbf{2} \Rightarrow \mathbf{2} \mathbf{i}-\mathbf{1}$ y $\mathbf{2 i - 1} \Rightarrow \mathbf{2 i}$ por lo que se tiene lo deseado para $1 \leq i \leq 2 n-1$.

(vii) Como $T\langle\mathbf{2} \mathbf{i}-\mathbf{1} \cup \mathbf{2} \mathbf{i}+\mathbf{1}\rangle \cong S R_{4}$ para $1 \leq i \leq 2 n-1$, entonces tenemos los triángulos

$$
\begin{aligned}
& \overrightarrow{C_{3}}: \quad(2 i-1,1) \rightarrow(2 i+1,1) \rightarrow(2 i+1,2) \rightarrow(2 i-1,1) \quad \mathrm{y} \\
& \overrightarrow{C_{3}}: \quad(2 i-1,1) \rightarrow(2 i-1,2) \rightarrow(2 i+1,2) \rightarrow(2 i-1,1) .
\end{aligned}
$$

(viii) Similar al caso anterior, $T\langle\mathbf{2} \mathbf{i} \cup \mathbf{2} \mathbf{i}+\mathbf{2}\rangle \cong S R_{4}$ para $1 \leq i \leq 2 n$, entonces tenemos los triángulos

$$
\begin{aligned}
& \overrightarrow{C_{3}}: \quad(2 i, 1) \rightarrow(2 i+2,1) \rightarrow(2 i+2,2) \rightarrow(2 i, 1) \quad \mathrm{y} \\
& \overrightarrow{C_{3}}: \quad(2 i, 1) \rightarrow(2 i, 2) \rightarrow(2 i+2,2) \rightarrow(2 i-1,1)
\end{aligned}
$$




\subsection{Inconexión acíclica y libre de $\vec{C}_{3}$ de los elemen- tos de la familia $\mathfrak{V}_{n}$}

Una vez construida la familia $\mathfrak{V}_{n}$, el paso siguiente es el de mostrar sus propiedades respecto a la inconexión acíclica e inconexión libre de $\overrightarrow{C_{3}}$, para finalmente dar respuesta afirmativa a los problemas mencionados al inicio del presente capítulo.

El siguiente teorema determina la inconexión libre de $\overrightarrow{C_{3}}$ de los miembros de la familia $\mathfrak{V}_{n}$ y nos proporciona una coloración que la realiza.

Teorema 2.1. Sea $T_{8 n+1} \in \mathfrak{V}_{n}(n \geq 1)$, entonces $\vec{\omega}_{3}\left(T_{8 n+1}\right)=n+2$.

Demostración. La prueba se sigue por inducción sobre $n$.

Para el caso $n=1$, tenemos que $\vec{\omega}_{3}\left(T_{9}\right)=3$ por la observación 2.1 de la página 26 .

Supongamos que el teorema es válido para $n-1$, es decir $\vec{\omega}_{3}\left(T_{8(n-1)+1}\right)=n+1 \mathrm{y}$ demostremos que $\vec{\omega}_{3}\left(T_{8 n+1}\right)=n+2$.

Procedamos por contradicción y supongamos que $\vec{\omega}_{3}\left(T_{8 n+1}\right) \geq n+3$, entonces existe una $(n+3)$-coloración libre de $\vec{C}_{3}$ heterocromáticos

$$
\psi: V\left(T_{8 n+1}\right) \rightarrow\left\{X_{1}, X_{2}, \ldots, X_{n+3}\right\} .
$$

Por hipótesis de inducción, tenemos que $\vec{\omega}_{3}\left(T_{8(n-1)+1}\right)=n+1$, por lo que a lo más podemos asignar $n+1$ colores en $V\left(T_{8(n-1)+1}\right)$. Sin pérdida de generalidad, supongamos que los colores $X_{n+2}$ y $X_{n+3}$ son los dos nuevos colores a asignar a los vértices de $T_{8 n+1}\langle\{4 \mathbf{n}-\mathbf{3}, \mathbf{4 n}-\mathbf{2}, \mathbf{4 n}-\mathbf{1}, \mathbf{4 n}\}\rangle$.

Por el lemma $2.1^{1}$ tenemos que $V(\langle\{\mathbf{4 n}-\mathbf{3}, \mathbf{4 n}-\mathbf{2}\}\rangle)$ y $V(\langle\{\mathbf{4 n}-\mathbf{1}, \mathbf{4 n}\}\rangle$ deben ser monocromáticos, así la única manera posible de asignar los nuevos colores es si definimos $\psi(\{\mathbf{4 n}-\mathbf{3}, \mathbf{4 n}-\mathbf{2}\})=X_{n+2}$ y $\psi(\{\mathbf{4 n} \mathbf{- 1}, \mathbf{4 n}\})=X_{n+3}$. Notemos que $\overrightarrow{C_{3}}: u \rightarrow v \rightarrow w \rightarrow u$ es heterocromático, donde $u \in \mathbf{4 n}-\mathbf{4}, v \in \mathbf{4 n - 2}$ y $w \in \mathbf{4 n}$, que es una contradicción a la suposición original.

Concluimos que $\vec{\omega}_{3}\left(T_{8 n+1}\right) \leq n+2$.

Por la definición de $T_{8 n+1} \in \mathfrak{V}_{n}$ y por lema $2.2^{2}$ tenemos que la coloración definida por

$$
\varphi(v)= \begin{cases}X_{1}, & \text { si } v=\{0\}, \\ X_{2}, & \text { si } v \in\{\mathbf{1}, \mathbf{2}\}, \\ X_{i+2}, & \text { si } v \in\{\mathbf{4 i - 1}, \mathbf{4 i}, \mathbf{4 i}+\mathbf{1}, \mathbf{4 i}+\mathbf{2}\}, 1 \leq i \leq n-1, \\ X_{n+2}, & \text { si } v \in\{\mathbf{4 n - 1}, \mathbf{4 n}\},\end{cases}
$$

es una $(n+2)$-coloración libre de $\overrightarrow{C_{3}}$ heterocromáticos. Por lo tanto la coloración $\varphi$ es óptima y hemos demostrado que $\vec{\omega}_{3}\left(T_{8 n+1}\right)=n+2$.

\footnotetext{
${ }^{1}$ página 27

${ }^{2}$ página 30
} 
De manera similar al teorema anterior, el siguiente teorema determina la inconexión acíclica de los elementos de la familia $\mathfrak{V}_{n}$.

Teorema 2.2. Sea $T_{8 n+1} \in \mathfrak{V}_{n}(n \geq 1)$, entonces $\vec{\omega}\left(T_{8 n+1}\right)=2$.

Demostración. La demostración se sigue por inducción sobre $n$.

El caso $n=1$ se sigue por la observación 2.1 de la página 26.

Supongamos que el teorema es válido para $n-1$, es decir, $\vec{\omega}\left(T_{8(n-1)+1}\right)=2$ y demostremos que $\vec{\omega}\left(T_{8 n+1}\right)=2$. Supongamos por contradicción que $\vec{\omega}\left(T_{8 n+1}\right) \geq 3$, por lo que existe una 3-coloración óptima

$$
\psi: V\left(T_{8 n+1}\right) \rightarrow\left\{X_{1}, X_{2}, X_{3}\right\}
$$

Por hipótesis de inducción, tenemos que $\vec{\omega}\left(T_{8(n-1)+1}\right)=2$, así solo podemos asignar dos colores a $V\left(T_{8(n-1)+1}\right)$. Sin pérdida de generalidad, supongamos que el color $X_{3}$ es el color que no ha sido asignado, entonces este color debe ser asignado a alguno de los vértices de $\left.T_{8 n+1}\langle\{\mathbf{4 n - 3}, \mathbf{4 n - 2}, \mathbf{4 n}-\mathbf{1}, \mathbf{4 n}\}\rangle\right)$. Por la observación 2.1, tenemos que

$$
T_{8 n+1}\langle\{0, \mathbf{4 n}-\mathbf{5}, \mathbf{4 n}-\mathbf{4}, \mathbf{4 n}-\mathbf{3}, \mathbf{4 n}-\mathbf{2}\}\rangle \quad \text { y } \quad T_{8 n+1}\langle\{0, \mathbf{4 n}-\mathbf{3}, \mathbf{4 n}-\mathbf{2}, \mathbf{4 n}-\mathbf{1}, \mathbf{4 n}\}\rangle
$$

son copias isomorfas de $T_{9} \in \mathfrak{V}_{n}$. Por el lema $2.1^{3}$, los conjuntos $V\left(T_{8 n+1}\langle\{\mathbf{4 n}-\mathbf{5}, \mathbf{4 n}-\mathbf{4}\})\right.$, $V\left(T_{8 n+1}\langle\{\mathbf{4 n - 3}, \mathbf{4 n - 2}\}\rangle\right)$ y $V\left(T_{8 n+1}\langle\{\mathbf{4 n} \mathbf{- 1}, \mathbf{4 n}\}\rangle\right)$ son monocromáticos.

Si $V\left(T_{8 n+1}\langle\{\mathbf{4 n - 5}, \mathbf{4 n - 4}\}\rangle\right) \cap X_{3}=\emptyset$, entonces $V\left(T_{8 n+1}\langle\{\mathbf{4 n - 3}, \mathbf{4 n - 2}\}\rangle\right) \cap X_{3}=\emptyset$, en caso contrario, el ciclo

$$
\overrightarrow{C_{4}}:(4 n-5,1) \rightarrow(4 n-3,1) \rightarrow(4 n-5,2) \rightarrow(4 n-3,2) \rightarrow(4 n-5,1)
$$

está bien coloreado. Así

$$
V\left(T_{8 n+1}\langle\{\mathbf{4 n - 3}, \mathbf{4 n - 2}\}\rangle\right) \cap\left(X_{1} \cup X_{2}\right) \neq \emptyset .
$$

Similarmente podemos demostrar que $V\left(T_{8 n+1}\langle\{\mathbf{4 n} \mathbf{- 1}, \mathbf{4 n}\}) \cap X_{3}=\emptyset\right.$, lo que es una contradicción.

Dado que $\vec{\omega}\left(T_{8 n+1}\right) \leq 2$ y como la coloración definida por

$$
\varphi(v)= \begin{cases}X_{1}, & \text { si } v=\{0\} \\ X_{2}, & \text { si } v \in V\left(T_{4 n+1}\right) \backslash\{0\}\end{cases}
$$

es libre de $\left\{\overrightarrow{C_{3}}, \overrightarrow{C_{4}}\right\}$ bien coloreados, entonces es óptima.

Notemos que la coloración (2.4) no es única, ya que existen muchas 2-coloraciones que proporcionan una coloración óptima, sin embargo nos permitimos tomarla así, para tener una coloración $\vec{\omega}$-aguda ya que esta será de gran utilidad más adelante.

\footnotetext{
${ }^{3}$ página 27
} 
Corolario 2.1. Para todo entero $s \geq 2$, existe un torneo $T$ tal que $\vec{\omega}(T)=2$ y $\vec{\omega}_{3}(T)=s$.

Demostración. Sea $T \in \mathfrak{V}_{n}$ y por los teoremas 2.1 y 2.2 se tiene lo deseado.

La familia $\mathfrak{V}_{n}$ proporciona un infinito número de ejemplos de torneos, para los cuales tenemos que la diferencia $\overrightarrow{\omega_{3}}(T)-\vec{\omega}(T)$ es tan grande como se desee, lo que contesta afirmativamente al problema $2.1^{4}$ del inicio de este capítulo.

De la observación $2.1^{5}$, se desprende que la coloración (2.4) es $\vec{\omega}$-aguda y la coloración (2.3) $\vec{\omega}_{3}$-aguda. Esta es una propiedad interesante que se repite en todo miembro de la familia $\mathfrak{V}_{n}$. Para demostrar esta propiedad haremos uso del siguiente resultado.

Lema 2.3. Sea $T$ un torneo. Si toda flecha $u \rightarrow v$ en $A(T)$ pertenece a un triángulo dirigido, entonces toda $\vec{\omega}_{3}(T)$-coloración óptima tiene a lo más una clase cromática singular.

Demostración. Por contradicción, supongamos que existe una $\vec{\omega}_{3}(T)$-coloración óptima que deja al menos dos clases cromáticas singulares. Sean $u$ y $v$ dos clases singulares, entonces existe $w$ en alguna otra clase cromática tal que $\vec{C}_{3}: w \rightarrow u \rightarrow v \rightarrow w$ es heterocromático. Esto es una contradicción .

Corolario 2.2. Todo torneo de la familia $\mathfrak{V}_{n}$ es $\vec{\omega}$-agudo y $\vec{\omega}_{3}$-agudo.

Demostración. Por la coloración definida en (2.4) del teorema 2.2, $T_{8 n+1}$ es $\vec{\omega}$-agudo. Similarmente, por el lema $2.2^{6}$, toda flecha $u \rightarrow v$ en $A\left(T_{8 n+1}\right)$ pertenece a un $\overrightarrow{C_{3}}$, por lo que al usar el lema 2.3 y la coloración definida en (2.3) en el teorema 2.1, tenemos que $T_{8 n+1}$ es $\vec{\omega}_{3}$-agudo.

El lema 2.3 y el corolario 2.2 nos permiten probar el siguiente teorema, que es una consecuencia directa para la familia $\mathfrak{V}_{n}$ y es un resultado de especial interés, ya que nos proporciona uno de los teoremas que consideramos de mayor relevancia en este trabajo y es una generalización del problema 6.6 en [NL99] y del corolario 2.1, para $\vec{\omega}(T)=r$, donde $2 \leq r \leq s$.

Teorema 2.3. Para todo par de enteros $(r, s)$ tal que $2 \leq r \leq s$, existe un torneo $T^{*}$ tal que $\vec{\omega}\left(T^{*}\right)=r$ y $\vec{\omega}_{3}\left(T^{*}\right)=s$.

Demostración. Sea $k=s-r$ y definamos $k=k_{1}+k_{2}+\ldots+k_{r-1}$, donde $k_{i}$ es un entero no negativo para toda $i \in\{1,2, \ldots, r-1\}$. Notemos que la función $\phi: \mathfrak{V}_{n} \rightarrow \mathbb{N} \backslash\{1,2\}$ $(n \geq 1)$ definida por $\phi\left(T_{8 n+1}\right)=n+2$, es una biyección.

\footnotetext{
${ }^{4}$ página 23

${ }^{5}$ página 26

${ }^{6}$ página 30
} 
Sea $\mathfrak{B}=\left\{B_{i}: i \in\{1,2, \ldots, r-1\}\right\}$ el conjunto de $r-1$ torneos, donde

$$
B_{i}= \begin{cases}\vec{C}_{3}, & \text { si } k_{i}=0 \\ T_{8 k_{i}+1} \in \mathfrak{V}_{n}, & \text { si } k_{i} \geq 1\end{cases}
$$

Definimos al torneo $T^{*}$, por una aplicación sucesiva de la composición de los elementos de $\mathfrak{B}$, esto es

$$
T^{*}=B_{1}\left[B_{2}\left[\cdots\left[B_{r-2}\left[B_{r-1}\right]\right] \cdots\right]\right] .
$$

Notemos que $\vec{\omega}\left(B_{i}\right)=2$ y $\vec{\omega}_{3}\left(B_{i}\right)=k_{i}+2$. Por el corolario 2.2 , tenemos que todo miembro de la familia $\mathfrak{V}_{n}$ es $\vec{\omega}$-agudo y $\vec{\omega}_{3}$-agudo, entonces

$$
\vec{\omega}\left(T^{*}\right)=\sum_{i=1}^{r-1} \vec{\omega}\left(B_{i}\right)-(r-2)=2(r-1)-(r-2)=r
$$

al aplicar $(r-1)$ veces el corolario $1.3^{7}$.

Similarmente,

$$
\vec{\omega}_{3}\left(T^{*}\right)=\sum_{i=1}^{r-1} \vec{\omega}_{3}\left(B_{i}\right)-(r-2)=\sum_{i=1}^{r-1}\left(k_{i}+2\right)-(r-2)=\sum_{i=1}^{r-1} k_{i}+r=s .
$$

Recordemos que dado un entero no negativo $r$, la ecuación $r=x_{1}+x_{2}+\ldots+x_{n}$, donde $x_{i}$ son enteros no negativos, tiene $\left(\begin{array}{c}n+r-1 \\ r\end{array}\right)$ soluciones distintas.

Como la composición de torneos no es conmutativa, entonces $T^{*}$ depende de la descomposición de $k$ como suma de los enteros no negativos $k_{1}, k_{2}, \ldots, k_{r-1}$. Por lo que el teorema 2.3 proporciona

$$
\left(\begin{array}{c}
k+r-2 \\
k
\end{array}\right)
$$

formas distintas de hacer la composición y por lo tanto, igual número de torneos que cumplen las condiciones, aunque no necesariamente todos ellos son distintos.

\footnotetext{
${ }^{7}$ página 14
} 


\section{Una conjetura sobre la tensión}

En [NL99], Víctor Neumann-Lara demuestra que si un torneo regular no es simple -es decir es isomorfo a una composición-, entonces no es tenso y conjetura que el recíproco es también cierto $([\mathrm{NL}])$. Esta conjetura es reforzada con los trabajos posteriores sobre familias infinitas simples que resultan ser tensas.

En este capítulo se da un primer acercamiento a la solución de dicha conjetura, para la cual definimos una familia infinita de torneos, cuyos elementos son simples pero no tensos. Esta familia demuestra que la conjetura no es cierta, sin embargo esta familia tiene la característica de que todos sus elementos son isomorfos a una suma de Zykov no trivial, es decir no son primos, por lo que hacemos una modificación a dicha conjetura en un sentido más general que la propuesta por Neumann-Lara.

\subsection{Pares discordantes}

Esta sección es una breve introducción a la teoría de gráficas de dominación, que junto con los trabajos [NLO09] y [LlO07], nos proporcionan una forma de definir un nuevo tipo de torneos regulares simples no tensos. En [CKL98], [CDKL98], [FLMR98] y [FL99] se pueden encontrar resultados recientes que versan sobre el tema.

Definición 3.1 ([NLO09]). Sean $T$ un torneo y $S \subset V(T)$. Decimos que $u, v \in V(D)$ son discordantes módulo $S$ y lo denotamos por $u \mid v$ (mód $S$ ), si

$$
\begin{aligned}
& N^{+}(u, S \backslash\{u, v\})=N^{-}(v, S \backslash\{u, v\}) \quad y \\
& N^{-}(u, S \backslash\{u, v\})=N^{+}(v, S \backslash\{u, v\}) .
\end{aligned}
$$

Definición 3.2. Sean $T$ un torneo $y\{u, v\} \subset V(T)$. Decimos que $\{u, v\}$ forman un par discordante si

$$
N^{+}(u, T) \cup N^{+}(v, T) \cup\{u, v\}=V(T) .
$$

Equivalentemente, un par discordante $\{u, v\}$ se puede definir como aquellos pares para los cuales

$$
N^{+}(u, T \backslash\{u, v\})=N^{-}(v, T \backslash\{u, v\}) .
$$

La siguiente proposición da una propiedad interesante de algunos torneos regulares, que tienen como subgráficas a otros torneos regulares de menor cardinalidad. Esta proposición garantiza que podemos obtener nuevos torneos regulares de mayor cardinalidad a partir de un torneo dado. Esta proposición nos será de mucha utilidad al construir más adelante a la familia $\mathfrak{U}$.

Lema 3.1 (Lema 6 en [NLO09]). Sean $T$ un torneo regular de orden $2 n+1$ y un par $\{u, v\} \subset V(T)$. Entonces el torneo residual $T^{\prime}=T\langle V(T) \backslash\{u, v\}\rangle$ es regular si y solo si u|v (mód $V(T))$. 
Demostración. Es claro que si eliminamos el par $\{u, v\}$ del conjunto de $V(T)$, a toda $w \in V(T) \backslash\{u, v\}$ le hemos quitado un ex-vecino y un in-vecino, es decir

$$
d^{+}\left(w, T^{\prime}\right)=d^{-}\left(w, T^{\prime}\right)=n-1,
$$

esto sucede si y solo si

$$
N^{+}\left(u, T^{\prime}\right)=N^{-}\left(v, T^{\prime}\right) \quad \text { y } \quad N^{-}\left(u, T^{\prime}\right)=N^{+}\left(v, T^{\prime}\right)
$$

si y solo si $u \mid v($ mód $V(T))$.

El lema 3.1 se puede reescribir como sigue.

Observación 3.1. Sea $T$ es un torneo regular de orden $(2 n+1)$ y $\{u, v\} \subset V(T)$. Entonces el torneo residual $T^{\prime}=T\langle V(T) \backslash\{u, v\}\rangle$ es regular si y solo si $\{u, v\}$ es un par discordante.

En la figura 3.1 mostramos los torneos circulantes $\vec{C}_{9}\langle\emptyset\rangle$ y $\vec{C}_{7}\langle\emptyset\rangle$. Notemos que el par $\{0,4\} \subset V\left(\vec{C}_{9}\langle\emptyset\rangle\right)$ es discordante y que

$$
\vec{C}_{9}\langle\emptyset\rangle\left\langle\mathbb{Z}_{9} \backslash\{0,4\}\right\rangle \cong \vec{C}_{7}\langle\emptyset\rangle
$$

Más aún, como el torneo circulante $\vec{C}_{2 n+1}\langle\emptyset\rangle$ es transitivo en vértices, entonces el par $\{i, n+i\}$ es discordante para toda $i \in \mathbb{Z}_{2 n+1}$ y se tiene que

$$
\vec{C}_{2 n+1}\langle\emptyset\rangle\left\langle\mathbb{Z}_{2 n+1} \backslash\{i, n+i\}\right\rangle \cong \vec{C}_{2 n-1}\langle\emptyset\rangle
$$

con el isomorfismo

$$
\phi: V\left(\vec{C}_{2 n+1}\langle\emptyset\rangle\left\langle\mathbb{Z}_{2 n+1} \backslash\{i, n+i\}\right\rangle\right) \rightarrow V\left(\vec{C}_{2 n-1}\langle\emptyset\rangle\right)
$$

definido por

$$
\phi(v)= \begin{cases}i-1, & \text { si } v \in\{1,2, \ldots, n-1\} \\ i-2, & \text { si } v \in\{n+1, n+2, \ldots, 2 n\} .\end{cases}
$$

El siguiente teorema fue dado en [LlO07], pero para este trabajo, proponemos una demostración alternativa, que nos ayudará a comprender la estructura de un torneo con un par discordante.

Teorema 3.1 (Teorema 4 en [LlO07]). Sean $T$ un torneo regular, $\{u, v\}$ un par discordante y $T^{\prime}=T\langle V(T) \backslash\{u, v\}\rangle$ el torneo residual. Si $T^{\prime}$ es tenso entonces $T$ también es tenso.

Demostración. Sea $T$ un torneo regular de orden $(2 n+1)$. Por el lema 3.1, se tiene que el torneo residual $T^{\prime}=T\langle V(T) \backslash\{u, v\}\rangle$ es un torneo regular de orden $2 n-1$. 

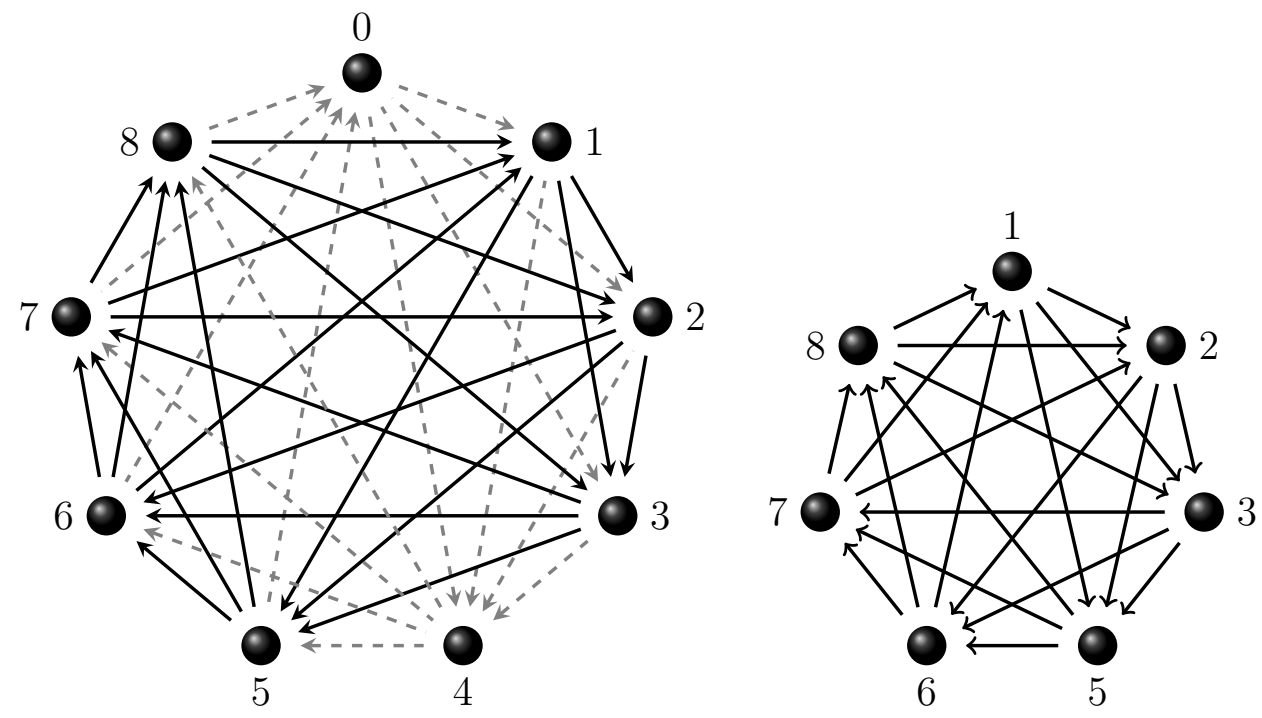

Figura 3.1: Los torneos $\vec{C}_{9}\langle\emptyset\rangle$ y $\vec{C}_{7}\langle\emptyset\rangle$.

Supongamos que $T^{\prime}$ es tenso y sea $\varphi: V\left(T^{\prime}\right) \rightarrow\{X, Y\}$ una 2-coloración óptima. Definamos los siguientes conjuntos para el color $X$ :

$$
\begin{aligned}
& P_{X}^{+}(u)=\left\{w \in V\left(T^{\prime}\right) \cap N^{+}(u, T): \varphi(w)=X\right\}, \\
& P_{X}^{-}(u)=\left\{w \in V\left(T^{\prime}\right) \cap N^{-}(u, T): \varphi(w)=X\right\} .
\end{aligned}
$$

De manera similar definimos los conjuntos $P_{Y}^{+}(u)$ y $P_{Y}^{-}(u)$ para el color $Y$.

Notemos que $P_{X}^{-}(u)=P_{X}^{+}(v), P_{X}^{+}(u)=P_{X}^{-}(v), P_{Y}^{-}(u)=P_{Y}^{+}(v)$ y $P_{Y}^{+}(u)=P_{Y}^{-}(v)$, además de ser ajenos dos a dos (véase la figura 3.2).

Supongamos por contradicción que $T$ no es tenso, es decir $\overrightarrow{\omega_{3}}(T) \geq 3$, por lo que existe una 3-coloración óptima $\psi: V(T) \rightarrow\{X, Y, Z\}$. Por hipótesis se tiene que ningún vértice de $T^{\prime}$ es de color $Z$, así $\psi(u)=Z$ o $\psi(v)=Z$. Sin pérdida de generalidad, supongamos que $u \rightarrow v$ y que $\psi(u)=Z$.

Observemos lo siguiente:

(i) Para toda $w_{1} \in P_{X}^{-}(u)$ y $w_{2} \in P_{Y}^{+}(u)$, se tiene que $w_{1} \rightarrow w_{2}$ en $V\left(T^{\prime}\right)$, en caso contrario $\overrightarrow{C_{3}}: u \rightarrow w_{2} \rightarrow w_{1} \rightarrow u$ sería heterocromático. Se tiene un caso similar para toda $w_{1} \in P_{Y}^{-}(u)$ y $w_{2} \in P_{X}^{+}(u)$.

(ii) Por construcción, $P_{X}^{-}(u)$ y $P_{X}^{+}(u)$ no pueden ser vacíos al mismo tiempo. Se tiene un caso similar para $P_{Y}^{-}(u)$ y $P_{Y}^{+}(u)$.

(iii) $P_{X}^{-}(u)$ y $P_{X}^{-}(u)$ no pueden ser vacíos al mismo tiempo, pues sería una contradicción a la regularidad de $T$. De manera similar se cumple para $P_{X}^{+}(u)$ y $P_{Y}^{+}(u)$. 


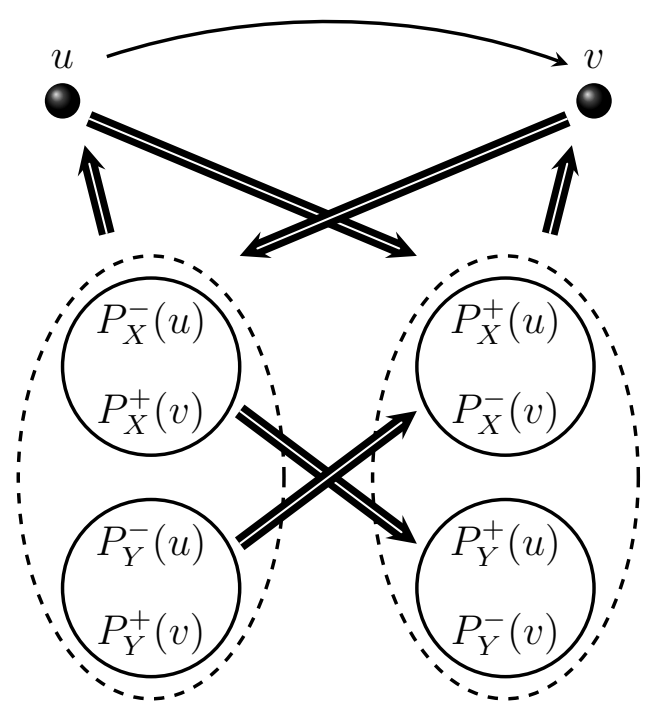

Figura 3.2: El torneo $T$ y el par discordante $\{u, v\}$.

(iv) Por los incisos (ii) y (iii) tenemos que

- Si $P_{X}^{-}(u)=\emptyset$ о $P_{Y}^{+}(u)=\emptyset$, entonces $P_{X}^{+}(u) \neq \emptyset$ y $P_{Y}^{-}(u) \neq \emptyset$.

- Si $P_{X}^{+}(u)=\emptyset$ о $P_{Y}^{-}(u)=\emptyset$, entonces $P_{X}^{-}(u) \neq \emptyset$ y $P_{Y}^{+}(u) \neq \emptyset$.

Por el inciso (iii), tenemos que $\psi(v) \notin\{X, Y\}$, en caso contrario tendríamos que el triángulo $\overrightarrow{C_{3}}: u \rightarrow v \rightarrow w \rightarrow u$ sería heterocromático para $w \in P_{X}^{-}(u)$ o $w \in P_{Y}^{-}(u)$. Por lo tanto $\psi(v)=Z$, pero esto es una contradicción a (iv), dado que para $w_{1} \in P_{X}^{-}(u)$, $w_{2} \in P_{X}^{+}(u), w_{3} \in P_{Y}^{-}(u)$ y $w_{4} \in P_{Y}^{+}(u)$, se tendría que $\overrightarrow{C_{3}}: v \rightarrow w_{1} \rightarrow w_{4} \rightarrow v$ o $\overrightarrow{C_{3}}: v \rightarrow w_{3} \rightarrow w_{2} \rightarrow v$ sería heterocromático.

Por lo que tal coloración no existe y se prueba que $T$ también es tenso. El caso $v \rightarrow u$ se demuestra de manera similar.

Definición 3.3. La gráfica de dominación $\mathfrak{D}(T)$ es definida sobre los conjuntos

$$
\begin{aligned}
& V(\mathfrak{D}(T))=V(T) \quad y \\
& A(\mathfrak{D}(T))=\{u \rightarrow v:\{u, v\} \text { es un par discordante }\} .
\end{aligned}
$$

Definición 3.4. Si $T$ es un torneo regular y toda trayectoria dirigida en $\mathfrak{D}(T)$ es de orden a lo más dos, entonces se dirá que $T$ es un molde. Un molde $M^{T}$ de un torneo circulante no cíclico $T$ es el torneo regular inducido por $V(T) \backslash\{u, v\}$.

Un molde se dirá manso si $T\langle V(T) \backslash\{u, v\}\rangle \cong \vec{C}_{2 m+1}\langle\emptyset\rangle$. Un torneo $T$ es manso si $M^{T}$ es manso. Si $T$ es cualquier torneo regular y toda trayectoria dirigida es de orden al menos tres en $\mathfrak{D}(T)$, entonces se dirá que $T$ es un torneo amplio.

El teorema 3.1 es un caso particular del expuesto en [LlO07], donde se demuestra la tensión de los torneos con moldes mansos (véase [NLO09]) y de los torneos amplios. 


\subsection{Familias de torneos regulares simples y tensos}

En el inciso (i) de la proposición $1.4^{1}$ (proposición 3.6 en [NL99]), se muestra que si una digráfica es una composición de dos digráficas $B$ y $D$, donde $B$ es $\overrightarrow{\omega_{3}}$-aguda entonces

$$
\vec{w}_{3}(B[D])=\vec{\omega}_{3}(B)+\vec{\omega}_{3}(D)-1
$$

Para el caso particular de torneos, si un torneo $T$ proviene de alguna composición, entonces no puede ser tensa, es decir, si un torneo es tenso, entonces es simple. Víctor Neumann-Lara conjeturó que el recíproco era también cierto, por lo que formuló la siguiente:

Conjetura 3.1 ([NL]). Todo torneo regular $T$ es simple si y solo si es tenso.

Los trabajos sobre algunas clases de torneos regulares tensos de H. Galeana-Sánchez, B. Llano, M. Olsen y del mismo V. Neumann-Lara en [NL99], [GSNL00], [LlO07] y [LINL07], reforzaron la sospecha de la veracidad de esta conjetura, ya que todos los ejemplos obtenidos de torneos que son tensos, provenían de familias cuyos elementos son simples, salvo las excepciones de torneos circulantes que resultan ser composiciones de torneos regulares circulantes y por lo tanto no son tensos.

La siguiente lista es una recopilación de las familias infinitas de torneos regulares, que fueron probadas tensas en los trabajos antes citados.

Observación 3.2. Las siguientes familias de torneos regulares son tensos:

(i) Los torneos cíclicos de cualquier orden, $\vec{C}_{2 n+1}\langle\emptyset\rangle$ (proposición 4.4 en [NL99]).

(ii) Los torneos circulantes obtenidos al voltear una flecha de su conjunto de saltos, excepto el caso cuando es una composición, esto es, $\vec{C}_{2 n+1}\langle s\rangle$ con $1 \leq s \leq n$, salvo el caso $(2 n+1, s)=(9,2)$ ya que $\vec{C}_{9}\langle 2\rangle \cong \overrightarrow{C_{3}}\left[\overrightarrow{C_{3}}\right]$ (teorema 4.11 en [NL99]).

(iii) Los torneos circulantes que se obtienen al voltear dos flechas de su conjunto de saltos, excepto los casos que resultan ser composiciones, esto es $\vec{C}_{2 n+1}\left\langle s_{1}, s_{2}\right\rangle$ con $1 \leq s_{1}<s_{2} \leq n$, excepto los casos

- $\left(2 n+1, s_{1}, s_{2}\right)=(9,1,4)$ y $\left(2 n+1, s_{1}, s_{2}\right)=(9,2,3)$, ya que

$$
\vec{C}_{9}\langle 1,4\rangle \cong \vec{C}_{9}\langle 2,3\rangle \cong \vec{C}_{3}\left[\overrightarrow{C_{3}}\right]
$$

- $\left(2 n+1, s_{1}, s_{2}\right)=(15,2,5)$ y $\left(2 n+1, s_{1}, s_{2}\right)=(15,3,4)$, ya que

$$
\vec{C}_{15}\langle 2,5\rangle \cong \vec{C}_{3}\left[\vec{C}_{5}\langle\emptyset\rangle\right] \quad \text { y } \quad \vec{C}_{15}\langle 3,4\rangle \cong \vec{C}_{5}\langle\emptyset\rangle\left[\vec{C}_{3}\right] .
$$

(teorema 5 en [GSNL00]).

\footnotetext{
${ }^{1}$ página 12
} 
(iv) Los torneos circulantes de orden un entero primo $p \geq 3$, esto es $\vec{C}_{p}(J)$ que resultan ser torneos primos (teorema 3 en [LlNLOr]).

(v) Los torneos circulantes simples, es decir, aquellos que no son isomorfos a composición alguna (teorema 4.10 en $[\mathrm{Ll}]$ ).

(vi) Los torneos con molde manso (corolario 2 en [LlO0r]).

(vii) Los torneos amplios (teorema 5 en [LlOOr]).

Notemos que los torneos de estas familias infinitas son en su mayoría circulantes y simples o bien, se obtienen a partir de torneos circulantes simples. Hasta el momento no se contaba con un solo ejemplo de algún torneo regular simple no tenso.

\subsection{Una familia de torneos regulares, simples y no tensos}

La observación 3.2 nos resume de manera precisa, los ejemplos de torneos regulares que se saben tensos y -como lo habíamos mencionado anteriormente- los únicos ejemplos que se conocen de torneos regulares no tensos, son las composiciones.

En esta sección construimos la familia infinita de torneos $\mathfrak{U}$, cuyos elementos son torneos regulares simples no tensos, así la conjetura no es cierta en este sentido. Sin embargo, dichos torneos resultan ser una suma de Zykov no trivial, por lo que modificamos la conjetura en un sentido más amplio que el propuesto por Neumann-Lara.

Construcción 3.1. Sean $T_{1}$ un torneo regular tenso de orden $2 n+1$ y $T_{2}$ un torneo regular de orden $2 m+1$. Sea $T_{1}\left[T_{2}\right]$ la composición de ambos torneos, lo que resulta en un torneo regular de orden $(2 n+1)(2 m+1)$.

Agreguemos un par de vértices $\{u, v\}$ a $V\left(T_{1}\left[T_{2}\right]\right)$ y construyamos el torneo regular $U$ de orden

$$
(2 n+1)(2 m+1)+2=2(2 n m+2 n+2 m+1)+1,
$$

definido sobre los conjuntos

$$
\begin{aligned}
& V(U)=V\left(T_{1}\left[T_{2}\right]\right) \cup\{u, v\}, \\
& A(U)=A\left(T_{1}\left[T_{2}\right]\right) \cup F_{1} \cup F_{2},
\end{aligned}
$$

donde $F_{1}$ es exactamente una de las flechas $u \rightarrow v$ o $\rightarrow \rightarrow$ y $F_{2}$ se obtiene de la siguiente manera.

Sea $W_{1}$ la subdigráfica de $T_{1}\left[T_{2}\right]$ tal que $W_{1} \cong T_{2}$ y sean $W_{2}$ y $W_{3}$ las subdigráficas inducidas por la partición no degenerada en dos partes de $V\left(T_{1}\left[T_{2}\right] \backslash W_{1}\right)$, tales que

$$
\left|W_{2}\right|= \begin{cases}2 n m+n-m-1, & \text { si } u \rightarrow v, \\ 2 n m+n-m, & \text { si } v \rightarrow u\end{cases}
$$


$y$

$$
\left|W_{3}\right|= \begin{cases}2 n m+n+m+1, & \text { si } u \rightarrow v, \\ 2 n m+n+m, & \text { si } v \rightarrow u\end{cases}
$$

Asi $V\left(T_{1}\left[T_{2}\right]\right)=V\left(W_{1}\right)\left|V\left(W_{2}\right)\right| V\left(W_{3}\right)$ como se describe anteriormente. Definimos

$$
F_{2}=\left\{u \rightarrow w, w \rightarrow v: w \in V\left(W_{1} \cup W_{2}\right)\right\} \cup\left\{w \rightarrow u, v \rightarrow w: w \in V\left(W_{3}\right)\right\},
$$

es decir, $F_{2}$ es el conjunto de flechas tal que $u \Rightarrow W_{1} \cup W_{2} y W_{3} \Leftarrow v$.

Notemos que $\{u, v\}$ es un par discordante definido por

$$
N^{+}(u, U)=N^{-}(v, U)=V\left(W_{1} \cup W_{2}\right)
$$

$\mathrm{y}$

$$
N^{-}(u, U)=N^{+}(v, U)=V\left(W_{3}\right)
$$

además

$$
d^{+}(u)=d^{-}(u)=d^{+}(v)=d^{-}(v)=2 n m+n+m+1 .
$$

Por el lema $3.1^{2}$, tenemos que $U$ es un torneo regular.

Definimos la familia $\mathfrak{U}$ como el conjunto de torneos regulares obtenidos por la construcción 3.1. En la figura 3.3 se muestra la construcción de esta familia para el caso donde fijamos la flecha $v \rightarrow u$.

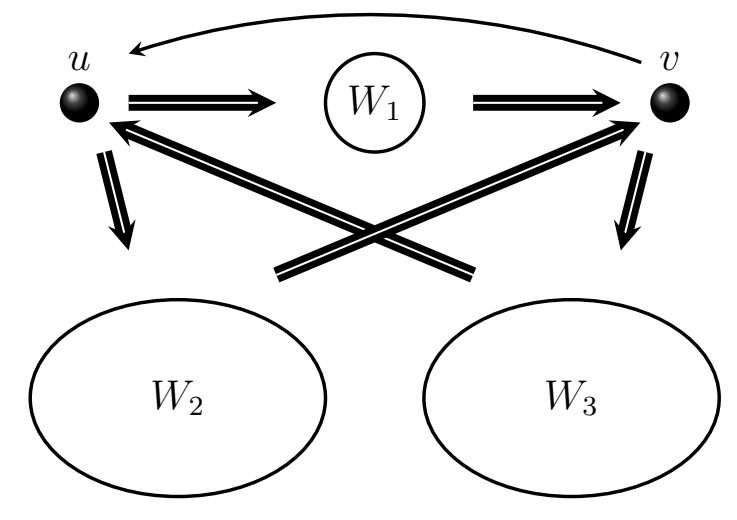

Figura 3.3: Construcción de la familia $\mathfrak{U}$.

En la figura 3.4 podemos observar la construcción del torneo $U_{1}$ que es uno de los elementos de menor orden de la familia $\mathfrak{U}$, donde fijamos la composición $\vec{C}_{3}\left[\vec{C}_{3}\right]$ y el par discordante $\{9,10\}$. Sea la partición $V\left(\vec{C}_{3}\left[\vec{C}_{3}\right]\right)=V\left(W_{1}\right)\left|V\left(W_{2}\right)\right| V\left(W_{3}\right)$ definida por

$$
\begin{aligned}
V\left(W_{1}\right) & =\{0,3,6\} \\
V\left(W_{2}\right) & =\{7,5\} \quad \mathrm{y} \\
V\left(W_{3}\right) & =\{1,2,4,8\}
\end{aligned}
$$

\footnotetext{
${ }^{2}$ página 37
} 
Finalmente agregamos las flechas apropiadas como se indica en la construcción 3.1 y obtenemos el torneo regular $U_{1} \in \mathfrak{U}$ de orden 11 .

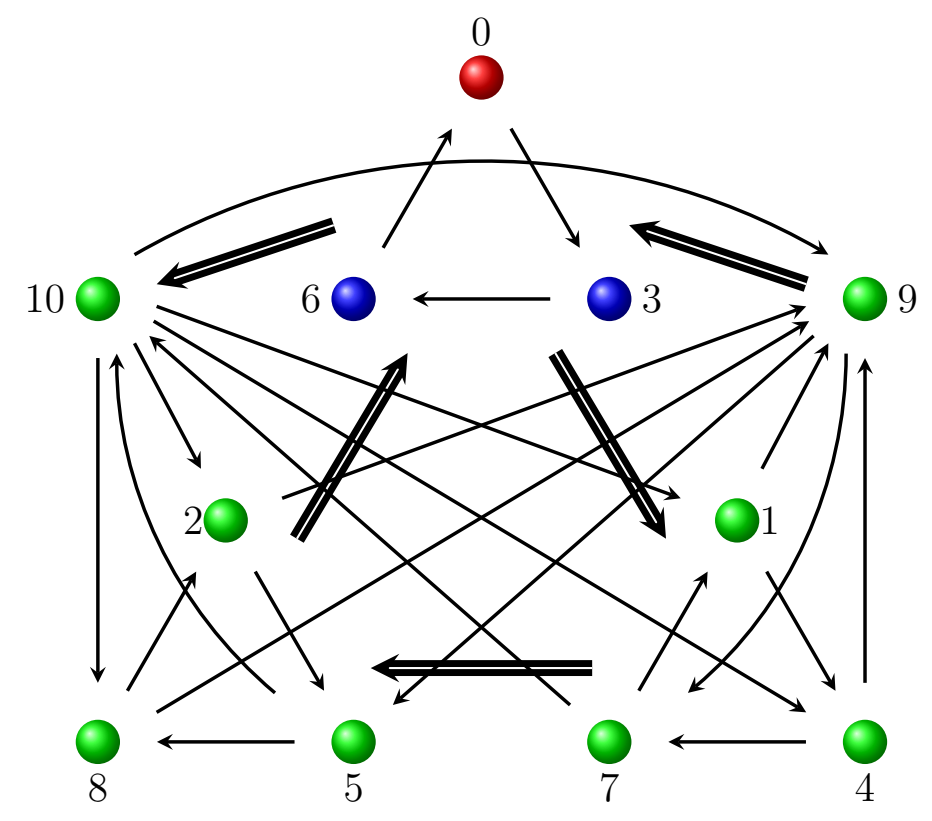

Figura 3.4: El torneo $U_{1} \in \mathfrak{U}$ con $\vec{C}_{3}\left[\vec{C}_{3}\right]$ y $\{u, v\}=\{9,10\}$.

Teorema 3.2. Sean $T_{1}$ y $T_{2}$ torneos regulares, tales que $T_{1}$ es tenso y $\overrightarrow{\omega_{3}}\left(T_{2}\right)=r-1$ $(r \geq 3)$. Sea $U \in \mathfrak{U}$ como en la construcción 3.1, entonces $\vec{\omega}_{3}(U)=r$.

Demostración. Las hipótesis del teorema cumplen los requisitos de la observación $1.3^{3}$, por lo que al hacer los cambios de variable $s:=2$ y $r:=r-1$ en la misma, la coloración (1.1) de esta observación, se reduce a la $r$-coloración

$$
\varphi: V\left(T_{1}\left[T_{2}\right]\right) \rightarrow\left\{X_{1}, Y_{1}, Y_{2}, \ldots, Y_{r-1}\right\}
$$

que es óptima y libre de $\vec{C}_{3}$ heterocromáticos. Así $\vec{\omega}_{3}(U) \geq r$.

Para $T_{1}\left[T_{2}\right]$, definamos la partición

$$
V\left(T_{1}\left[T_{2}\right]\right)=V\left(W_{1}\right)\left|V\left(W_{2}\right)\right| V\left(W_{3}\right)
$$

como en la construcción 3.1. Supongamos que $\pi\left(V\left(W_{1}\right)\right)$ es el vértice singular en $T_{1}$, donde $\pi$ es el epimorfismo reflexivo $\pi: V\left(T_{1}\left[T_{2}\right]\right) \rightarrow V\left(T_{1}\right)$ de la definición $1.7^{4}$, por lo que $W_{1} \cong T_{2}$. Por la observación 1.3 , tenemos que que la $r$-coloración es tal que $\varphi\left(W_{1}\right)=\left\{Y_{1}, Y_{2}, \ldots, Y_{r-1}\right\}$ y $\varphi\left(W_{2} \cup W_{3}\right)=X_{1}$.

\footnotetext{
${ }^{3}$ página 13

${ }^{4}$ página 11
} 
Por hipótesis, tenemos que es imposible agregar un nuevo color tanto en $V\left(W_{1}\right)$ como en $V\left(W_{2} \cup W_{3}\right)$. Supongamos por contradicción que $\vec{\omega}_{3}(U) \geq r+1$, así existe

$$
\psi: V(U) \rightarrow\left\{X_{1}, Y_{1}, \ldots, Y_{r}\right\}
$$

una $(r+1)$-coloración libre de $\vec{C}_{3}$ heterocromáticos. Sin pérdida de generalidad supongamos que $Y_{r}$ es el nuevo color que se asigna, por lo que este nuevo color debe ser asignado a $\{u, v\}$. Tenemos dos casos para la flecha inducida $T\langle\{u, v\}\rangle$.

Caso $v \rightarrow u$. Si $\psi(u)=Y_{r}$, entonces $\psi(v) \neq X_{1}$, ya que en caso contrario el triángulo $\vec{C}_{3}: u \rightarrow w \rightarrow v \rightarrow u$ sería heterocromático, para toda $w \in V\left(W_{1}\right)$.

Por otra parte, $\psi(v) \neq Y_{i}$ para toda $i \in\{1,2, \ldots, r-1\}$, ya que en caso contrario $\vec{C}_{3}: u \rightarrow w \rightarrow v \rightarrow u$ sería heterocromático para toda $w \in V\left(W_{2}\right)$. Por lo tanto $\psi(v)=Y_{r}$, pero esto es imposible, ya que dados $x \in W_{3}$ y $y \in W_{1}$

- si $x \rightarrow y$ entonces, $\vec{C}_{3}: x \rightarrow y \rightarrow v \rightarrow x$ es heterocromático,

- si $y \rightarrow x$ entonces $\vec{C}_{3}: x \rightarrow u \rightarrow y \rightarrow x$ es heterocromático,

ambos casos nos llevan a una contradicción a la suposición que $\psi(u)=Y_{r}$. De manera similar se prueba que $\psi(v) \neq Y_{r}$.

Caso $u \rightarrow v$. Tenemos dos subcasos.

- Si $\psi(u)=Y_{r}$, entonces $W_{1} \Leftarrow W_{3}$, ya que si existieran $y \in W_{1}$ y $x \in W_{3}$ tales que $y \rightarrow x$, entonces $\vec{C}_{3}: u \rightarrow y \rightarrow x \rightarrow u$ sería heterocromático. Por otra parte, notemos que para toda $w \in V\left(W_{1}\right)$, se tiene que $V\left(W_{3}\right) \cup\{u\} \subseteq N^{-}(w)$ y como $\left|W_{3}\right|=2 n m+n+m+1$, entonces

$$
d^{-}(w) \geq 2 n m+n+m+2,
$$

una contradicción a la regularidad de $U$. Esto demuestra que $\psi(u) \neq Y_{r}$.

- Si $\psi(v)=Y_{r}$, entonces $W_{1} \Rightarrow W_{3}$, ya que si existieran $y \in W_{1}$ y $x \in$ $W_{3}$ tales que $x \rightarrow y$, entonces el triángulo $\vec{C}_{3}: v \rightarrow x \rightarrow y \rightarrow v$ sería heterocromático. Por otra parte, notemos que para toda $w \in V\left(W_{1}\right)$, se tiene que $V\left(W_{3}\right) \cup\{v\} \subseteq N^{+}(w)$, por lo que similarmente al caso anterior, conduce a que

$$
d^{+}(y) \geq 2 n m+n+m+2
$$

que es una contradicción.

Por los dos casos anteriores tenemos que $\psi(v) \neq Y_{r}$.

Las contradicciones anteriores señalan que es imposible asignar el nuevo color $Y_{r}$, por lo que la coloración $\psi$ no existe y así $\vec{\omega}_{3}(T) \leq r$. Finalmente notemos que si

$$
\phi: V\left(T_{1}\right) \rightarrow\left\{Y_{1}, Y_{2}, \ldots, Y_{r-1}\right\}
$$


es una coloración óptima y libre de $\overrightarrow{C_{3}}$ heterocromáticos restringida a $W_{1} \cong T_{1}$, como en la observación 1.3, entonces la coloración

$$
\varphi(w)= \begin{cases}\phi(w), & \text { si } w \in V\left(W_{1}\right), \\ Y_{0}, & \text { si } w \in V\left(W_{2} \cup W_{3}\right) \cup\{u, v\},\end{cases}
$$

es libre de $\overrightarrow{C_{3}}$ heterocromáticos, ya que los nuevos triángulos que se generan contienen la flecha $u \rightarrow v$ (o $v \rightarrow u$ según el caso) y como $\varphi(u)=\varphi(v)$, entonces no pueden ser heterocromáticos. Así esta $r$-coloración es óptima.

El siguiente teorema es el equivalente del teorema anterior para la inconexión acíclica $\vec{\omega}$ y cuya demostración se sigue de manera similar.

Teorema 3.3. Si $\vec{\omega}\left(T_{1}\left[T_{2}\right]\right)=r$ entonces $\vec{\omega}(U)=r$,

Demostración. Como $\vec{\omega}(U) \leq \vec{\omega}_{3}(U)=r$, mostremos que $\vec{\omega}(U)$ se alcanza en $r$. Por la observación $1.4^{5}$, para calcular $\vec{\omega}(U)$ basta con probar que existe una $r$-coloración libre de $\left\{\vec{C}_{3}, \vec{C}_{4}\right\}$ bien coloreados. La coloración descrita en (3.1) del teorema 3.2 es libre de $\vec{C}_{3}$ heterocromáticos. Probemos que también es libre de $\vec{C}_{4}$ bien coloreados.

Notemos que los nuevos $\vec{C}_{4}$ que se forman al agregar los vértices $\{u, v\}$ a $T_{1}\left[T_{2}\right]$, son aquellos que los contienen y no pueden estar bien coloreados, ya que $\psi(u)=\psi(v)$. Así, la coloración $(3.1)$ es libre de $\left\{\overrightarrow{C_{3}}, \overrightarrow{C_{4}}\right\}$ bien coloreados.

Los teoremas 3.2 y 3.3 señalan que los elementos de la familia $\mathfrak{U}$ no son tensos, por lo que la pregunta natural es si estos torneos son simples. Tenemos el siguiente resultado que contesta esta cuestión.

Teorema 3.4. Sea $U \in \mathfrak{U}$, entonces $U$ es simple.

Demostración. Supongamos que $U$ es de orden $2 n+1$. Si $2 n+1$ es un número primo, entonces el resultado es inmediato, ya que no puede ser una composición, pues si lo fuese, su orden sería un número compuesto.

Procedamos por contradicción. Supongamos que $U$ no es primo, por lo que existen torneos regulares

$$
T_{1}=\left(V\left(T_{1}\right), A\left(T_{1}\right)\right) \quad \text { y } \quad T_{2}=\left(V\left(T_{2}\right), A\left(T_{1}\right)\right)
$$

de órdenes $2 n_{1}+1$ y $2 n_{2}+1$ respectivamente, tales que $U \cong T_{1}\left[T_{2}\right]$ y

$$
2 n+1=\left(2 n_{1}+1\right)\left(2 n_{2}+1\right)
$$

es una factorización de $2 n+1$ en enteros no necesariamente primos. Sea $\pi$ la proyección $\pi: V\left(T_{1}\left[T_{2}\right]\right) \rightarrow V\left(T_{1}\right)$ de la definición 1.7 y denotemos por $B_{i}$ a la subdigráfica de $U$ tal que $\pi\left(V\left(B_{i}\right)\right)=i$, es decir $B_{i} \cong T_{2}$ para toda $i \in V\left(T_{1}\right)$.

\footnotetext{
${ }^{5}$ página 21
} 
Sea $\{u, v\}$ un par discordante como en la construcción 3.1 de la familia $\mathfrak{U}$. Notemos que el par $\{u, v\}$ no puede estar contenido en $B_{i} \cong T_{2}$ alguna, para toda $i \in V\left(T_{1}\right)$. En caso contrario, dada $w \in V(U) \backslash B_{i}$ se tendría que $w \Rightarrow\{u, v\}$ o $w \Leftarrow\{u, v\}$ que es claramente una contradicción al hecho de ser un par discordante.

Supongamos que $u$ y $v$ pertenecen a copias distintas de $T_{2}$, por lo que existen $i, j \in V\left(T_{1}\right)$ con $i \neq j$, tales que $u \in V\left(B_{i}\right)$ y $v \in V\left(B_{j}\right)$. Como $B_{i} \cong B_{j}$, entonces existe una biyección

$$
\theta: V\left(B_{i}\right) \rightarrow V\left(B_{j}\right)
$$

tal que para toda $v^{\prime} \in V\left(B_{i}\right)$, existe una única $v \in V\left(B_{j}\right)$ tal que $\theta\left(v^{\prime}\right)=v$. Esto es imposible, ya que $\left\{u, v^{\prime}\right\}$ también sería un par discordante y es una contradicción similar a la anterior.

El teorema 3.4 da respuesta negativa a la conjetura 3.1, ya que los torneos regulares de la familia $\mathfrak{U}$ son simples y no son tensos. Sin embargo es posible obtener una suma de Zykov no trivial que caracteriza los elemenos de la familia. El siguiente teorema muestra lo anterior.

Teorema 3.5. Sea $U \in \mathfrak{U}$ como en la construcción 3.1, entonces $U$ es una suma de Zykov no trivial.

Demostración. Sea $T_{1}\left[T_{2}\right]$ un torneo de orden $(2 n+1)(2 m+1)$, obtenido por la composición de los torneos $T_{1}$ y $T_{2}$ de órdenes $(2 n+1)$ y $(2 m+1)$ respectivamente. Sea $\{u, v\}$ un par discordante y $V\left(T_{1}\left[T_{2}\right]\right)=V\left(W_{1}\right)\left|V\left(W_{2}\right)\right| V\left(W_{3}\right)$ una partición como en la construcción 3.1. Es claro que

$$
W_{1} \Rightarrow N^{+}\left(V\left(W_{1}\right)\right) \cap\left(W_{2} \cup W_{3}\right)
$$

y que

$$
W_{1} \Leftarrow N^{-}\left(V\left(W_{1}\right)\right) \cap\left(W_{2} \cup W_{3}\right) .
$$

Notemos en la figura 3.3 que $u \Rightarrow W_{1}$ y $W_{1} \Rightarrow v$, por lo que si $\pi: V\left(T_{1}\left[T_{2}\right]\right) \rightarrow V\left(T_{1}\right)$ es el epimorfismo reflexivo y lo aplicamos al conjunto $T_{2} \cong W_{1}$, obtenemos un torneo $B$ de orden $2 n(2 m+1)+3$, al cual tomaremos como la base de la suma de Zykov.

Finalmente, obtenemos la suma de Zykov

$$
B\left[\left\{T_{2},(2 n(2 m+1)+3) \cdot K_{1}\right\}\right]
$$

es decir, el torneo $U$ puede ser representado como una suma de Zykov, cuya base es el torneo $B$ y cuyo vértice $w=\pi\left(T_{2}\right)$ tiene asociado la componente $T_{2}$ y los restantes vértices tienen asociados vértices singulares.

En el teorema anterior, hemos definido a la suma de Zykov en términos de la base $B$ y la familia

$$
\alpha=\left\{T_{2},(2 n(2 m+1)+3) \cdot K_{1}\right\}
$$


Esta familia no necesariamente es minimal, en el sentido del menor número de miembros de la familia $\alpha$, ya que por cada uno de los subconjunto de vértices

$$
\begin{aligned}
& S_{1} \subset V\left(W_{2}\right) \cap N^{+}\left(W_{1}\right), \\
& S_{2} \subset V\left(W_{2}\right) \cap N^{-}\left(W_{1}\right), \\
& S_{3} \subset V\left(W_{3}\right) \cap N^{+}\left(W_{1}\right) \quad \mathrm{y} \\
& S_{4} \subset V\left(W_{3}\right) \cap N^{-}\left(W_{1}\right),
\end{aligned}
$$

tales que $T\left\langle S_{i}\right\rangle \cong T_{2}$, podemos aplicar el epimorfismo reflexivo $\pi: V\left(T_{1}\left[T_{2}\right]\right) \rightarrow V\left(T_{1}\right)$, a cada una de estas componentes y la base $B$ tendrá un vértice asociado a cada una de las copias de $T_{2}$.

En la figura 3.4 de la página 44, hemos construido el torneo regular de once vértices perteneciente a la familia $\mathfrak{U}$, con $T^{\prime} \cong \vec{C}_{3}\left[\vec{C}_{3}\right]$, el par discordante $\{9,10\}$ y la partición $V\left(\vec{C}_{3}\left[\vec{C}_{3}\right]\right)=V\left(W_{1}\right)\left|V\left(W_{2}\right)\right| V\left(W_{3}\right)$ definida por

$$
\begin{aligned}
V\left(W_{1}\right) & =\{0,3,6\}, \\
V\left(W_{2}\right) & =\{7,5\} \mathrm{y} \\
V\left(W_{3}\right) & =\{1,2,4,8\} .
\end{aligned}
$$

Sobre esta partición, la base $B$ de la suma de Zykov $B[\alpha]$, es el torneo de orden nueve cuyo conjunto de vértices es

$$
V(B)=V\left(\vec{C}_{3}\left[\vec{C}_{3}\right]\right) \backslash\{0,3,6\} \cup \pi(\{0,3,4\}) \cup\{9,10\},
$$

donde $\pi: V\left(\overrightarrow{C_{3}}\left[\overrightarrow{C_{3}}\right]\right) \rightarrow V\left(\overrightarrow{C_{3}}\right)$. La familia $\alpha$ está integrada por un $\vec{C}_{3}$ y ocho vértices singulares, es decir

$$
\alpha=\left\{\vec{C}_{3}, 8 \cdot K_{1}\right\}
$$

Sin embargo esta partición no es minimal, ya que si tomamos la misma base $\overrightarrow{C_{3}}\left[\overrightarrow{C_{3}}\right]$ y construimos el torneo $U_{2}$ con la partición $V\left(\vec{C}_{3}\left[\vec{C}_{3}\right]\right)=V\left(W_{1}\right)\left|V\left(W_{2}\right)\right| V\left(W_{3}\right)$ con

$$
\begin{aligned}
V\left(W_{1}\right) & =\{0,3,6\}, \\
V\left(W_{2}\right) & =\{1,4\} \mathrm{y} \\
V\left(W_{3}\right) & =\{2,5,7,8\},
\end{aligned}
$$

que se muestra en la figura 3.5, entonces la base $B$ es el torneo de orden 7 , con

$$
V(B)=V\left(\vec{C}_{3}\left[\vec{C}_{3}\right]\right) \backslash\{0,2,3,5,6,8\} \cup \pi(\{0,3,4\}) \cup \pi(\{2,5,8\}) \cup\{9,10\}
$$

y la familia $\alpha$ está integrada por dos copias de $\vec{C}_{3}$ y cinco vértices singulares, es decir

$$
\alpha=\left\{2 \cdot \vec{C}_{3}, 5 \cdot K_{1}\right\}
$$

Es claro que si tomamos un torneo $U$ como en la construcción 3.1, el cual es una suma de Zykov no trivial y aplicamos inductivamente la construcción 3.1 al torneo resultante 


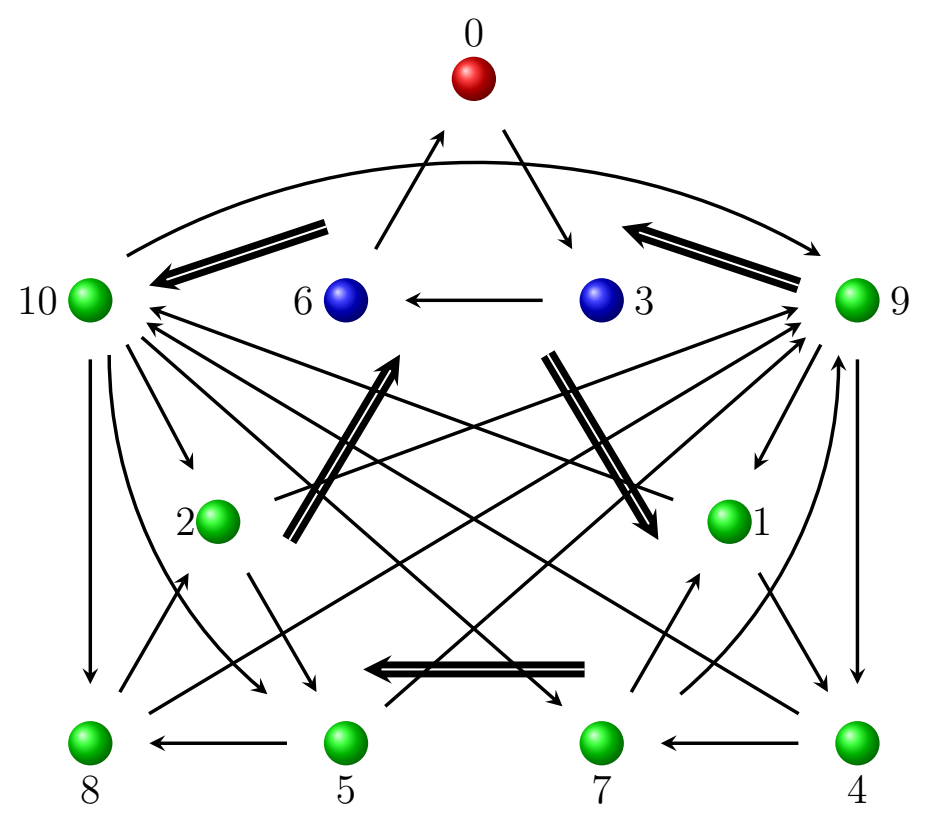

Figura 3.5: El torneo $U_{2}$ con $\vec{C}_{3}\left[\vec{C}_{3}\right]$ y $\{u, v\}=\{9,10\}$.

un número arbitrario de veces, obtenemos un torneo de mayor orden e isomorfo a una nueva suma de Zykov no trivial. Considerando este hecho, tenemos el siguiente teorema. Antes consideremos algunas definiciones previas.

Recordemos que dado un entero positivo $m$, por el Teorema Fundamental de la Aritmética, es posible descomponerlo como un producto de potencias de factores primos de la forma $m=\prod_{i=1}^{k} p_{i}^{q_{i}}$.

Sea

$$
\eta(n)=\operatorname{máx}_{3 \leq m \leq n}\left\{\sum_{i=1}^{k} q_{i}: m=\prod_{i=1}^{k} p_{i}^{q_{i}}, p_{i} \geq 3 \text { y primo }\right\} .
$$

Es decir, $\eta(n)$ será la mayor suma de los exponentes, de entre todas las factorizaciones en potencias de primos distintos de 2 , de los números menores o iguales que $n$. Por ejemplo, si $n=79$ entonces $\eta(79)=3$, debido a que entre todas las factorizaciones de los números menores o iguales a él, la máxima suma de sus exponentes se alcanza en 3 , ya que $27=3^{3}, 45=\left(3^{2}\right)(5), 63=\left(3^{2}\right)(7)$ y $75=(3)\left(5^{2}\right)$.

Teorema 3.6. Para toda $r \in\{1,2, \ldots, \eta(2 n+1)\}$, existe un torneo regular $T$ de orden $2 n+1$ que es isomorfo a una suma de Zykov, tal que $\vec{\omega}_{3}(T)=\vec{\omega}(T)=r+1$.

Demostración. Sea $m=\prod_{i=1}^{k} p_{i}^{q_{i}} \leq 2 n+1$ un entero tal que $q_{1}+q_{2}+\ldots+q_{k}=\eta(2 n+1)$. Definamos el multiconjunto

$$
M_{m}=\left\{q_{1} \cdot p_{1}, q_{2} \cdot p_{2}, \ldots, q_{k} \cdot p_{k}\right\} .
$$


Sea una partición $P=P_{1}\left|P_{2}\right| \cdots \mid P_{r}$ de $M_{m}$ en $r$ partes, con $1 \leq r \leq \eta(2 n+1)$ y para toda $i \in\{1,2, \ldots, r\}$ definimos el torneo regular tenso $T_{i}$ de orden $\prod_{p_{j} \in P_{i}} p_{j}$ de alguna de las formas descritas en la observación $3.2^{6}$. Definimos el torneo base

$$
B=T_{1}\left[T_{2}\left[\ldots\left[T_{r}\right]\right] \ldots\right] .
$$

Como $\overrightarrow{\omega_{3}}\left(T_{i}\right)=\vec{\omega}\left(T_{i}\right)=2$ para toda $i \in\{1,2, \ldots, r\}$, entonces por el corolario $1.3^{7}$, obtenemos que

$$
\vec{\omega}_{3}(B)=\vec{\omega}(B)=\sum_{i=1}^{r} \vec{\omega}\left(T_{i}\right)-(r-1)=2 r-(r-1)=r+1 .
$$

Es claro que el torneo $B$ tiene orden $m=\prod_{i=1}^{k} p_{i}^{q_{i}} \leq 2 n+1$.

Agreguemos un número par de vértices $2 n+1-m$ al torneo $B$ para definir el torneo $T$, como aquel construido a partir de $B$ al aplicar inductivamente $\frac{2 n+1-m}{2}$ veces el procedimiento de la construcción 3.1. Como $\overrightarrow{\omega_{3}}(B)=\vec{\omega}(B)=r+1$ entonces por los teoremas 3.2 y 3.3 , tenemos que $\overrightarrow{\omega_{3}}(T)=\vec{\omega}(T)=r+1$.

Como ejemplo del teorema anterior, sea el entero 19, el cual, de entre todas las factorizaciones de los enteros menores que él, solo $9=3^{2}$ y $15=(3)(5)$ son los que tienen la mayor suma de sus exponentes, de entre todas las factorizaciones de los enteros menores a ellos, por lo que $\eta(19)=2$, es decir $r \in\{1,2\}$.

Para $r=1$, el resultado es trivial al definir el torneo $T$ de orden 19, de alguna de las formas descritas en la observación 3.2 y claramente $\vec{\omega}_{3}(T)=\vec{\omega}(T)=2$.

Para $r=2$, tenemos que $m \in\{9,15\}$ y definimos los multiconjuntos $M_{9}=\{2 \cdot 3\}$ y $M_{15}=\{1 \cdot 3,1 \cdot 5\}$.

Para el caso $m=9$, sea el torneo de orden $9, B=\vec{C}_{3}\left[\vec{C}_{3}\right]$, entonces apliquemos la construcción 3.1 cinco veces, por lo que el torneo regular resultante es orden 19.

Para el caso $m=15$, tenemos la partición $M_{15}=\{3\} \mid\{5\}$ o $M_{15}=\{5\} \mid\{3\}$. Sea el torneo de orden $15, B=\vec{C}_{3}\left[\vec{C}_{5}\langle\emptyset\rangle\right]$ o $B=\vec{C}_{5}\langle\emptyset\rangle\left[\vec{C}_{3}\right]$ respectivamente y apliquemos la construcción 3.1 dos veces.

Claramente cualquiera de las formas anteriores nos proporcionan un torneo regular $T$ de orden 19, tal que $\vec{\omega}_{3}(T)=\vec{\omega}(T)=3$.

Por todo lo anterior, se sigue que un torneo simple $T$ no necesariamente es tenso, por lo que la conjetura 3.3 es falsa en este sentido. Sin embargo dicha conjetura la modificamos de una manera más general considerando sumas de Zykov.

Conjetura 3.2 (Segunda versión de la conjetura 3.1). Sea $T$ un torneo regular. Entonces $T$ es primo si y solo si es tenso.

\footnotetext{
${ }^{6}$ página 41

${ }^{7}$ página 14
} 


\section{Torneos regulares tensos y no tensos}

En el capítulo anterior se expuso una generalización de la conjetura 3.1, en la que se consideran clases más generales de torneos que no necesariamente son simples. En este capítulo, se da respuesta afirmativa a la conjetura 3.2, lo que se traduce en una caracterización para los torneos regulares primos.

Para las secciones posteriores, haremos uso de los siguientes resultados sobre ciclos en torneos multipartitos.

\subsection{Ciclos en torneos multipartitos}

Recordemos que una $r$-coloración de los vértices del torneo $T$, es una partición no degenerada en $r$ partes $V(T)=X_{1}\left|X_{2}\right| \ldots \mid X_{r}$, por lo que en lo subsecuente al hablar de una $r$-coloración

$$
\varphi: V(T) \rightarrow\left\{X_{1}, X_{2}, \ldots, X_{r}\right\}
$$

estaremos en el entendido que $X_{i}$ representa tanto a la $i$-ésima clase cromática, como a los vértices pertenecientes a la $i$-ésima parte.

Definición 4.1. Un torneo multipartito es una orientación de la gráfica multipartita completa simple.

En particular, si el torneo multipartito tiene solo dos partes, entonces lo llamaremos simplemente torneo bipartito. Similarmente, si el torneo tiene solo tres partes, entonces lo llamaremos torneo tripartito. En general, si el torneo multipartito tiene $r \geq 2$ partes, entonces es un torneo $r$-partito.

Dados un torneo $T$ y $\varphi: V(T) \rightarrow\left\{X_{1}, X_{2}, \ldots, X_{r}\right\}$ una $r$-coloración de $V(T)$, podemos definir un torneo $r$-partito $M$, al tomar cada clase cromática como cada una de las $r$ partes y su conjunto de flechas es el conjunto inducido de flechas entre las clases cromáticas distintas, es decir $M=(V(M), A(M))$ está definido por

$$
\begin{aligned}
& V(M)=V(T), \\
& A(M)=A(T) \backslash\{A(T\langle X\rangle) \cup A(T\langle Y\rangle) \cup A(T\langle Z\rangle)\} .
\end{aligned}
$$

En la figura 4.1 mostramos una 3-coloración óptima del torneo $\vec{C}_{3}\left[\vec{C}_{3}\right]$ y al torneo tripartito inducido por las tres clases cromáticas.

Para probar el teorema principal de este capítulo, necesitamos de la ayuda de un teorema excepcionalmente útil y de gran elegancia: el teorema de Goddard-KubickiOellermann-Tian (véase [GKO91]).

Teorema 4.1 (Teorema 1 en [GKO91]). Sea $M=(V, A)$ un torneo $r$-partito $(r \geq 3)$. Entonces $M$ contiene un $\overrightarrow{C_{3}}$ si y solo si existe un ciclo en $M$ que contiene vértices de al menos tres de las partes. 

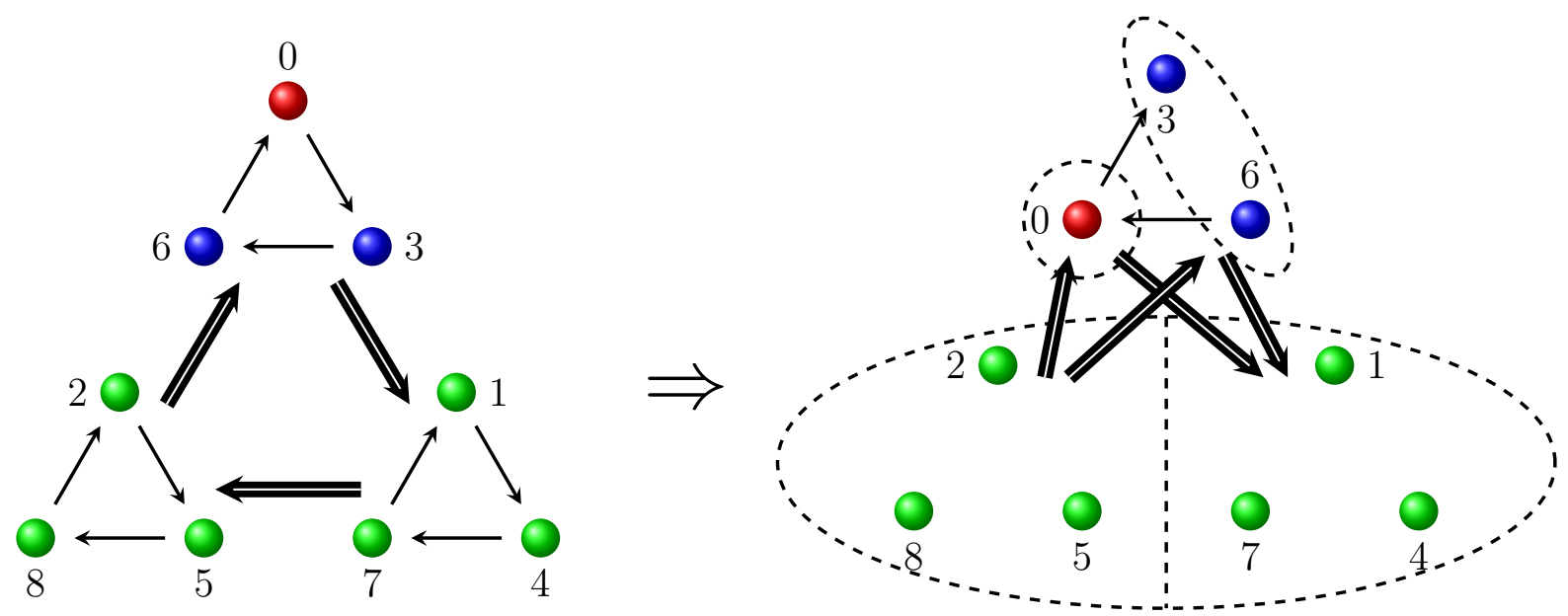

Figura 4.1: El torneo $\vec{C}_{3}\left[\vec{C}_{3}\right]$ y su torneo tripartito asociado.

Demostración. La necesidad es evidente, ya que si $M$ contiene un $\overrightarrow{C_{3}}$, entonces este $\overrightarrow{C_{3}}$ debe pasar por tres de las partes.

Para demostrar la suficiencia, supongamos por contradicción que $M$ es un torneo $r$-partito que contiene un ciclo $\vec{C}$ de longitud al menos cuatro, con vértices contenidos en al menos tres de las partes y que $M$ no induce $\overrightarrow{C_{3}}$ alguno. Supongamos que $\vec{C}$ es de longitud mínima.

Para demostrar el teorema haremos uso de la siguiente afirmación.

Afirmación 4.1. Existen $x \in X, y \in Y y z \in Z$ en particiones distintas tales que $x \rightarrow y \rightarrow z$ es una trayectoria de longitud tres contenida en $\vec{C}$.

Demostración. Supongamos que no existen trayectorias de longitud tres que pasen por las partes $X, Y$ y $Z$ consecutivamente, entonces las trayectorias que pasan por estas partes tienen longitud mayor a cuatro. Sea

$$
P: x_{1} \rightarrow y_{1} \rightarrow x_{2} \rightarrow y_{2} \rightarrow \ldots \rightarrow x_{l} \rightarrow y_{l} \rightarrow z
$$

una $x_{1} \rightsquigarrow z$ trayectoria de longitud mínima $l$ que pasa por las tres partes, donde $l \geq 2$, $\left\{x_{1}, x_{2}, \ldots, x_{l}\right\} \subseteq X,\left\{y_{1}, y_{2}, \ldots, y_{l}\right\} \subseteq Y$ y $z \in Z$.

Tenemos los dos casos siguientes:

- Si $z \rightarrow x_{l}$, entonces $x_{l} \rightarrow y_{l} \rightarrow z \rightarrow x_{l}$ es un $\vec{C}_{3}$, lo cual es imposible.

- Si $x_{l} \rightarrow z$, entonces

$$
P^{\prime}: x_{1} \rightarrow y_{1} \rightarrow x_{2} \rightarrow y_{2} \rightarrow \ldots \rightarrow x_{l} \rightarrow z
$$

es una trayectoria de menor longitud que $P$. Esto es una contradicción a la suposición de la minimalidad de la longitud de $P$. 
Ambos casos no pueden ser, por lo tanto hemos probado la afirmación.

De lo anterior, supongamos que los vértices de la trayectoria $x \rightarrow y \rightarrow z$ contenida en $\vec{C}$ no inducen un $\overrightarrow{C_{3}}$, donde $x \in X, y \in Y$ y $z \in Z$. Entonces $x \rightarrow z$ y notemos que

$$
\overrightarrow{C^{\prime}}=(\vec{C} \backslash\{y\}) \cup\{x \rightarrow z\}
$$

es un ciclo de menor longitud que $\vec{C}$ y pasa solo por las partes $X$ y $Z$, ya que si pasara por $Y$ entonces $\vec{C}$ t tendría longitud menor que $\vec{C}$. Lo anterior señala que $V\left(\vec{C}^{\prime}\right)$ induce un torneo bipartito. $\overrightarrow{C_{3}}$. Sea

Notemos que $x^{\prime} \in X$ es tal que $y \rightarrow x^{\prime}$, en caso contrario $x^{\prime} \rightarrow y \rightarrow z \rightarrow x^{\prime}$ sería un

$$
\vec{C}^{\prime \prime}=(\vec{C} \backslash\{z\}) \cup\left\{y \rightarrow x^{\prime}\right\}
$$

y notemos que es un ciclo que pasa por las tres partes y tiene longitud menor que la de $\vec{C}$, una contradicción.

Esto completa la demostración del teorema.

Este teorema proporciona dos corolarios muy útiles, que en el lenguaje de triángulos y ciclos contenidos en las clases cromáticas, los podemos traducir como siguen.

Corolario 4.1. Sean $T$ un torneo de orden $n \geq 3)$ y $M$ el torneo $r$-partito $(3 \leq r \leq n)$ inducido por una partición de $V(T)$ en $r$ partes. Entonces $M$ es externamente libre de $\overrightarrow{C_{3}}$ si y solo si es externamente acíclico.

Demostración. Se sigue directamente del teorema 4.1.

Corolario 4.2. Sean $T$ un torneo de orden $n \geq 3$ y $\varphi: V(T) \rightarrow\left\{X_{1}, X_{2}, \ldots, X_{r}\right\}$ una $r$-coloración, con $3 \leq r \leq n$. Entonces $T$ es libre de $\overrightarrow{C_{3}}$ heterocromáticos si y solo si es libre de ciclos bien coloreados.

Demostración. Se sigue directamente de teorema 4.1 y del corolario 4.1.

\subsection{Trayectorias prohibidas en torneos no tensos}

El lema y el corolario siguientes, a pesar de su sencillez, nos serán de utilidad más adelante al calcular cardinalidades de las segundas vecindades de un torneo regular.

Lema 4.1. Sean $T$ un torneo regular de orden $2 n+1$ y $v \in V(T)$, entonces

$$
N^{+}\left(N^{+}(v)\right) \backslash N^{+}(v)=N^{-}(v) .
$$

Demostración. Supongamos sin pérdida de generalidad que

$$
N^{+}(v)=\left\{v_{1}, v_{2}, \ldots, v_{n}\right\} \quad \text { y } \quad N^{-}(v)=\left\{v_{n+1}, v_{n+2}, \ldots, v_{2 n}\right\} .
$$


Por el lema $1.1^{1}$, tenemos que toda flecha de $V(T)$ está en un $\overrightarrow{C_{3}}$ dirigido, por lo que para toda $i \in\{1,2, \ldots, n\}$ existe $j \in\{n+1, n+2, \ldots, 2 n\}$, tal que la flecha $v \rightarrow v_{i}$ está en un $\overrightarrow{C_{3}}: v \rightarrow v_{i} \rightarrow v_{j} \rightarrow v$. Por lo tanto

$$
N^{+}\left(N^{+}(v)\right) \backslash N^{+}(v) \subseteq N^{-}(v) .
$$

Similarmente, tenemos que para toda $j \in\{n+1, n+2, \ldots, 2 n\}$, la flecha $v_{j} \rightarrow v$ está en un $\overrightarrow{C_{3}}: v \rightarrow v_{i} \rightarrow v_{j} \rightarrow v$, por lo que

$$
N^{+}\left(N^{+}(v)\right) \backslash N^{+}(v) \supseteq N^{-}(v) .
$$

Con ambas contenciones obtenemos lo deseado.

Corolario 4.3. Sea $T$ un torneo regular de orden $2 n+1$, entonces

$$
\left|N^{+}\left(N^{+}(v)\right) \backslash N^{+}(v)\right|=n
$$

para toda $v \in V(T)$.

Demostración. Se sigue directamente del lema anterior.

Ahora estamos en la posición de demostrar el siguiente lema, cuya demostración no es en absoluto trivial. Este lema tiene una gran importancia, ya que permite demostrar la tensión de los torneos regulares primos.

Como ya mostramos en el teorema 4.1 , si existe una $r$-coloración libre de $\vec{C}_{3}$, entonces podemos asociarle un torneo $r$-partito, al tomar cada clase cromática como cada parte del torneo multipartito. Más aún, este torneo multipartito contiene un triángulo dirigido si y solo si contiene un ciclo que pasa por al menos tres de las clases cromáticas.

Lema 4.2. Sean $T$ un torneo regular y $\varphi: V(T) \rightarrow\{X, Y, Z\}$ una 3-coloración libre de $\overrightarrow{C_{3}}$ heterocromáticos. Supongamos que $|X| \leq|Y| \leq|Z|$, entonces

$$
N^{+}\left(N^{+}\left(N^{+}(X) \cap Y\right) \cap Z\right) \cap X=\emptyset
$$

$y$

$$
N^{+}\left(N^{+}\left(N^{+}(Y) \cap X\right) \cap Z\right) \cap Y=\emptyset .
$$

Demostración. Sea $\varphi: V(T) \rightarrow\{X, Y, Z\}$ una 3-coloración (no necesariamente óptima) libre de $\overrightarrow{C_{3}}$ heterocromáticos, tal que $1 \leq|X| \leq|Y| \leq|Z|$. Supongamos sin pérdida de generalidad que $1 \leq|X| \leq\left\lfloor\frac{2 n+1}{3}\right\rfloor \mathrm{y}$

- $2 \leq|Y| \leq\left\lfloor\frac{2 n+1}{3}\right\rfloor$, si $|T| \equiv 0,1$ (mód 3) o

\footnotetext{
${ }^{1}$ página 7
} 
- $2 \leq|Y| \leq\left\lceil\frac{2 n+1}{3}\right\rceil$, si $|T| \equiv 2($ mód 3$)$,

Supongamos por contradicción que

$$
N^{+}\left(N^{+}\left(N^{+}(X) \cap Y\right) \cap Z\right) \cap X \neq \emptyset .
$$

Sea $x \in N^{+}\left(N^{+}\left(N^{+}(X) \cap Y\right) \cap Z\right) \cap X$, entonces $x \in N^{+}\left(N^{+}\left(N^{+}(X) \cap Y\right) \cap Z\right)$ y $x \in X$, así existe $z \in N^{+}\left(N^{+}(X) \cap Y\right) \cap Z$ tal que la flecha $z \rightarrow x$ está en $A(T)$, por lo tanto $z \in N^{+}\left(N^{+}(X) \cap Y\right)$ y $z \in Z$. De manera similar, existe $y \in N^{+}(X) \cap Y$ tal que $y \rightarrow z$ y por lo tanto $y \in N^{+}(X)$ y $y \in Y$. Finalmente existe $x^{\prime} \in X$ tal que $x^{\prime} \rightarrow y$.

Hemos construido la trayectoria

$$
P_{1}: x^{\prime} \rightarrow y \rightarrow z \rightarrow x .
$$

Observemos en la figura 4.2 que $x \neq x^{\prime}$ en la trayectoria $P_{1}$, pues en caso contrario tendríamos que $\vec{C}_{3}: x \rightarrow y \rightarrow z \rightarrow x$ es heterocromático. Más aún, lo anterior señala que $2 \leq|X| \leq|Y| \leq|Z|$ y por lo tanto no existen clases cromáticas singulares.

Sea $M$ el torneo tripartito inducido por las clases cromáticas $X, Y$ y $Z$ como en la figura 4.2 .

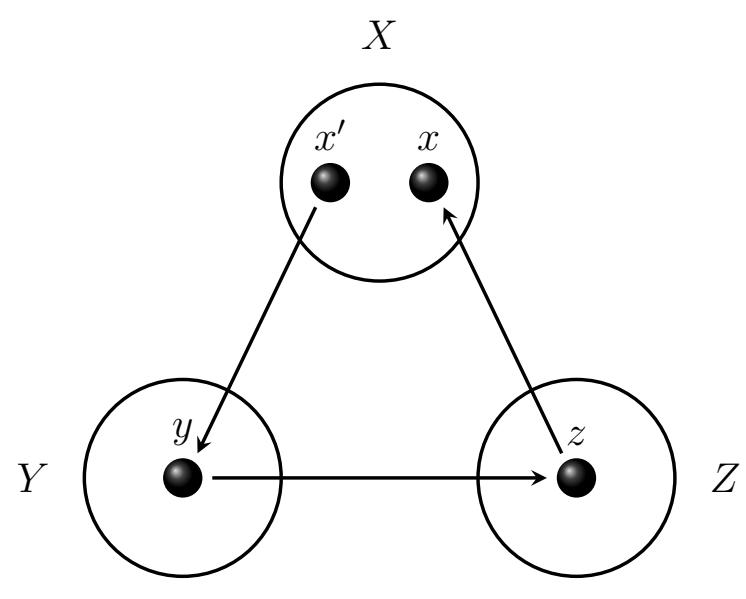

Figura 4.2: La trayectoria $P_{1}$.

Sea $x^{\prime}$ el vértice de la trayectoria $P_{1}$ y dado un conjunto $S \subseteq V(T)$, por simplicidad denotamos como $S^{+}=N^{+}\left(x^{\prime}\right) \cap S$ y $S^{-}=N^{-}\left(x^{\prime}\right) \cap S$.

Sea

$$
V(T)=\left\{x^{\prime}\right\}\left|X^{+}\right| X^{-}\left|Y^{+}\right| Y^{-}\left|Z^{+}\right| Z^{-}
$$

la partición inducida por las clases cromáticas y las in-vecindades y ex-vecindades de $x^{\prime}$ y notemos que

$$
\begin{aligned}
& N^{+}\left(x^{\prime}\right)=X^{+} \cup Y^{+} \cup Z^{+} \\
& N^{-}\left(x^{\prime}\right)=X^{-} \cup Y^{-} \cup Z^{-}
\end{aligned}
$$


y como es una partición libre de $\overrightarrow{C_{3}}$ heterocromáticos, entonces

$$
Z^{-} \Rightarrow Y^{+} \quad \text { y } \quad Y^{-} \Rightarrow Z^{+} .
$$

Así $y \in Y^{+}$y por lo tanto $z \in Z^{+}$. Notemos que $x \in X^{+}$o $x \in X^{-}$, por lo que supongamos sin pérdida de generalidad que $x \in X^{+}$y el caso $x \in X^{-}$se demuestra similarmente (véase la figura 4.3 con el caso $x \in X^{+}$). Consideremos los siguientes casos para extender la trayectoria $P_{1}$, los cuales dependen del último vértice de la misma.

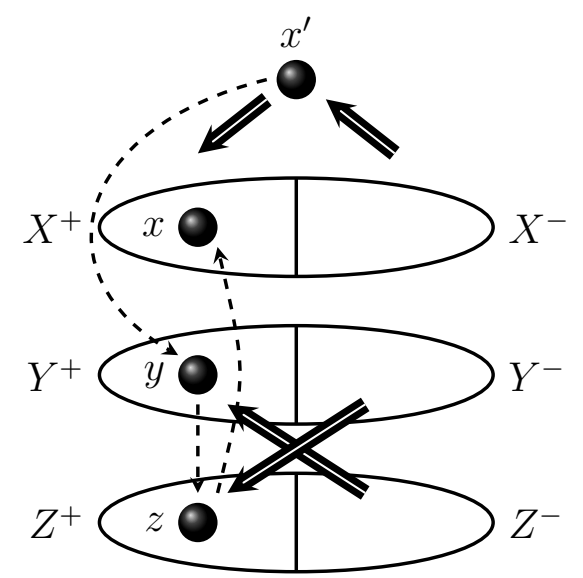

Figura 4.3: La partición con respecto a $x^{\prime}$ y la trayectoria $P_{1}$.

1. Observemos que

$$
Y^{-} \cup Z^{-} \Rightarrow x
$$

en caso contrario, si existe $u \in Y^{-} \cup Z^{-}$tal que $x \rightarrow u$, entonces el ciclo

$$
x^{\prime} \rightarrow y \rightarrow z \rightarrow x \rightarrow u \rightarrow x^{\prime}
$$

pasa por tres partes y por el teorema 4.1, existe un $\overrightarrow{C_{3}}$ heterocromático.

Afirmación 4.2. Sea la trayectoria $P_{1}$, entonces existe $y^{\prime} \in Y^{+} o z^{\prime} \in Z^{+}$tal que

$$
P_{2}: \underbrace{x^{\prime} \rightarrow y \rightarrow z \rightarrow x}_{P_{1}} \rightarrow y^{\prime}
$$

o bien

$$
P_{3}: \underbrace{x^{\prime} \rightarrow y \rightarrow z \rightarrow x}_{P_{1}} \rightarrow z^{\prime}
$$

es una trayectoria en $T$. 
Demostración. Supongamos por contradicción que $Y^{+} \cup Z^{+} \Rightarrow x$, entonces por (4.1), $Y \cup Z \Rightarrow x$ y tendríamos que $N^{+}(x) \subseteq X \backslash\{x\}$, por lo que

$$
\left|N^{+}(x)\right| \leq|X|-1<\left\lfloor\frac{2 n+1}{3}\right\rfloor<n,
$$

lo cual es una contradicción a la regularidad de $T$.

Para una trayectoria del tipo $P_{2}$, es claro que $y^{\prime} \neq y$ (en otro caso el triángulo $\overrightarrow{C_{3}}: y \rightarrow z \rightarrow x \rightarrow y^{\prime}=y$ sería heterocromático), y para una trayectoria del tipo $P_{3}$ tenemos que $z^{\prime} \neq z$, ya que en otro caso $x \leftrightarrow z^{\prime}=z$, que es una contradicción.

La figura 4.4 muestra este caso.

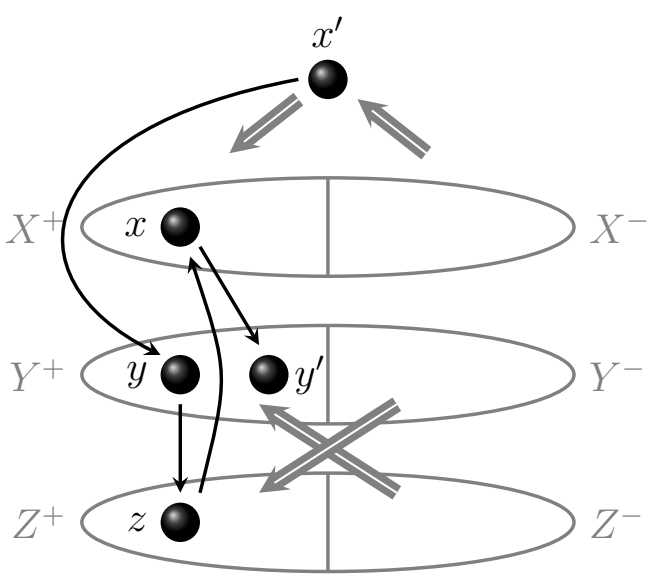

a) Trayectoria $P_{2}$.

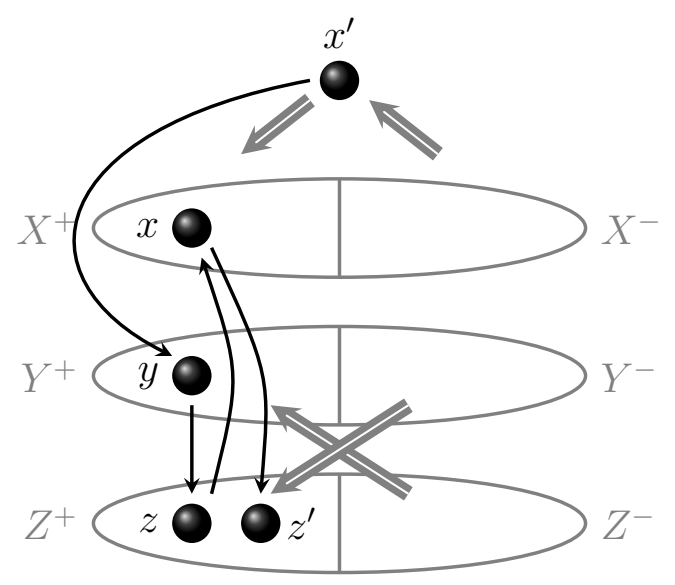

b) Trayectoria $P_{3}$.

Figura 4.4: Trayectorias de la afirmación 4.2.

Notemos que podemos generalizar la afirmación anterior y solo analizar los casos relativos al vértice final de la trayectoria $P$.

Afirmación 4.3. En general, si existe una trayectoria $P$ cuya subtrayectoria inicial es $P_{2}$ o $P_{3}$ y cuyo vértice final es un elemento $x^{\prime \prime} \in X$, es decir de la forma

$$
P_{2}^{\prime}: \underbrace{x^{\prime} \rightarrow y \rightarrow z \rightarrow x \rightarrow y^{\prime}}_{P_{2}} \rightarrow \cdots \rightarrow x^{\prime \prime}
$$

o bien

$$
P_{3}^{\prime}: \underbrace{x^{\prime} \rightarrow y \rightarrow z \rightarrow x \rightarrow z^{\prime}}_{P_{3}} \rightarrow \cdots \rightarrow x^{\prime \prime},
$$

entonces existe $y^{\prime \prime} \in Y^{+}$o bien, existe $z^{\prime \prime} \in Z^{+}(x)$ tal que $P$ se puede extender a la trayectoria

$$
P_{2}^{\prime \prime}: \underbrace{x^{\prime} \rightarrow y \rightarrow z \rightarrow x \rightarrow y^{\prime} \rightarrow \cdots \rightarrow x^{\prime \prime}}_{P_{2}^{\prime}} \rightarrow y^{\prime \prime}
$$


o bien a la trayectoria

$$
P_{3}^{\prime \prime}: \underbrace{x^{\prime} \rightarrow y \rightarrow z \rightarrow x \rightarrow z^{\prime} \rightarrow \cdots \rightarrow x^{\prime \prime}}_{P_{3}^{\prime}} \rightarrow z^{\prime \prime},
$$

donde $u \notin\{y, z\}$ para toda $u \in V(P) \backslash V\left(P_{1}\right)$,

Demostración. Sea $P$ de la forma $P_{2}^{\prime}$ o $P_{3}^{\prime}$. Observe que si $y$ o $z$ coincidiera con alguna $u \in V(P)$, tendríamos un ciclo que pasa por las tres partes y por el teorema 4.1, existe un $\vec{C}_{3}$ heterocromático.

Sea $S=V(P) \cap X$ y al igual que en el caso de la trayectoria $P_{1}$, observemos que $Y^{-} \cup Z^{-} \Rightarrow S$, en otro caso, tenemos un ciclo que pasa por las tres partes y por el teorema 4.1 contiene un $\overrightarrow{C_{3}}$ heterocromático.

Supongamos por contradicción que no es posible extender la trayectoria $P$ a alguna trayectoria de la forma $P_{2}^{\prime}$ o de la forma $P_{3}^{\prime}$. De lo anterior se sigue que $N^{+}\left(x^{\prime \prime}\right) \subseteq X \backslash\left\{x^{\prime \prime}\right\}$, por lo que

$$
\left|N^{+}\left(x^{\prime \prime}\right)\right| \leq|X|-1<\left\lfloor\frac{2 n+1}{3}\right\rfloor<n,
$$

lo cual es una contradicción a la regularidad de $T$.

Por lo tanto, si $P$ es una trayectoria cuyo vértice final es un elemento de $X$, es decir, del tipo $P_{2}^{\prime}$ o $P_{3}^{\prime}$, entonces siempre es posible extender la trayectoria hacia algún elemento de $Y^{+} \cup Z^{+}$. Con esto se termina la demostracion de la afirmación.

La figura 4.5 muestra este caso.

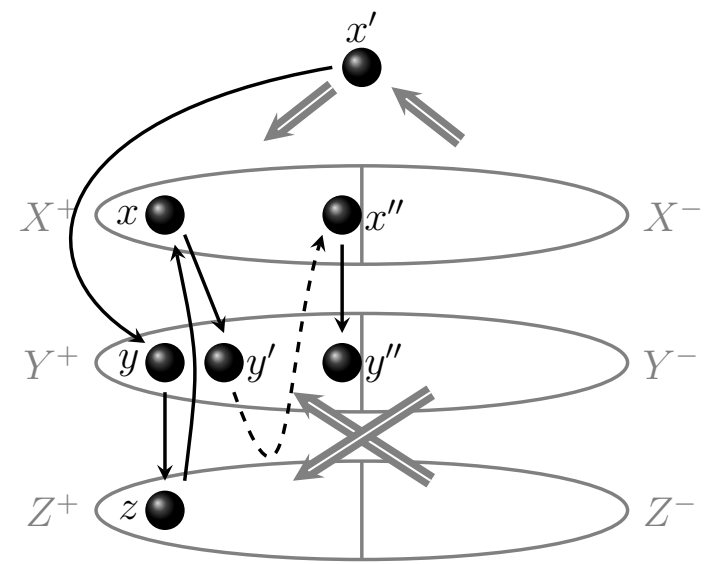

a) Trayectoria $P_{2}^{\prime}$.

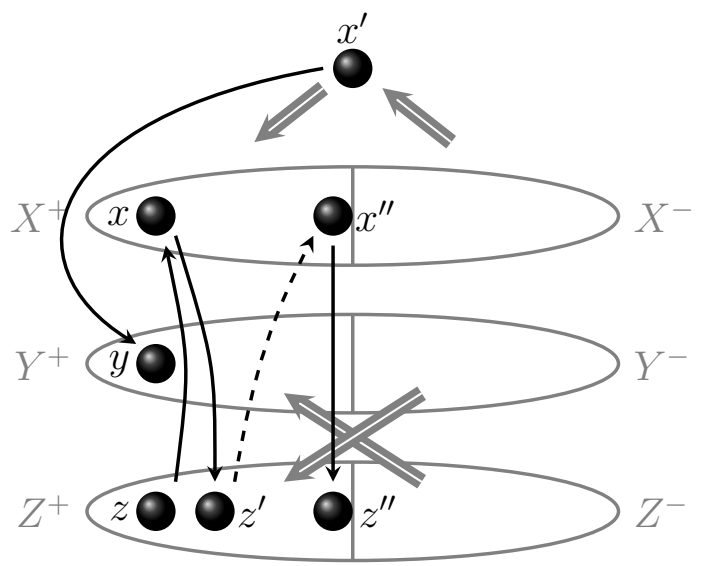

b) Trayectoria $P_{3}^{\prime}$.

Figura 4.5: Trayectorias de la afirmación 4.3.

Notemos que este caso se reduce a analizar solo el último vértice de la trayectoria $P$ y así podemos asegurar que es posible extender dicha trayectoria. Los siguientes casos retoman esta idea y solo consideran al último vértice de la trayectoria $P$. 
2. Si $P$ es un trayectoria cuyo vértice final es un elemento de $Y$, tenemos la siguiente.

Afirmación 4.4. Si $P$ es una trayectoria de la forma

$$
P_{4}: \underbrace{x^{\prime} \rightarrow y \rightarrow z \rightarrow x \rightarrow y^{\prime}}_{P_{2}} \rightarrow \cdots \rightarrow y^{\prime \prime}
$$

o bien de la forma

$$
P_{5}: \underbrace{x^{\prime} \rightarrow y \rightarrow z \rightarrow x \rightarrow z^{\prime}}_{P_{3}} \rightarrow \cdots \rightarrow y^{\prime \prime}
$$

entonces existe $x^{\prime \prime} \in X^{+} \cup X^{-}$o $z^{\prime \prime} \in Z^{+}$tal que la trayectoria $P$ puede ser extendida a una trayectoria del tipo

$$
P_{4}^{\prime}: \underbrace{x^{\prime} \rightarrow y \rightarrow z \rightarrow x \rightarrow \cdots \rightarrow y^{\prime \prime}}_{P_{4}} \rightarrow x^{\prime \prime}
$$

o bien

$$
P_{5}^{\prime}: \underbrace{x^{\prime} \rightarrow y \rightarrow z \rightarrow x \rightarrow \cdots \rightarrow y^{\prime \prime}}_{P_{5}} \rightarrow z^{\prime \prime}
$$

Demostración. De manera similar al caso anterior, tenemos que $u \notin\{y, z\}$ para toda $u \in V(P) \backslash V\left(P_{1}\right)$, pues en caso contrario tendríamos un ciclo que pasa por tres de las partes y por el teorema 4.1 , tenemos un $\overrightarrow{C_{3}}$ heterocromático. Notemos que $y^{\prime \prime} \notin Y^{-}$, pues en caso contrario, la trayectoria $P$ junto con la flecha $y^{\prime \prime} \rightarrow x^{\prime}$ formarían un ciclo que pasa por las tres clases cromáticas, una contradicción. Por lo tanto $y^{\prime \prime} \in Y^{+}$y así $X^{-} \cup Z^{-} \Rightarrow V(P) \cap Y^{+}$.

Supongamos que es imposible extender la trayectoria $P$ hacia algún elemento de $X \cup Z$, entonces $N^{+}\left(y^{\prime \prime}\right) \subseteq Y \backslash\left\{y^{\prime \prime}\right\}$, por lo que

$$
\left|N^{+}\left(y^{\prime \prime}\right)\right| \leq|Y|-1<\left\lfloor\frac{2 n+1}{3}\right\rfloor<n,
$$

lo cual es una contradicción a la regularidad de $T$. Por lo tanto si $P$ es una trayectoria con vértice final un elemento de $Y$, entonces siempre es posible extender la trayectoria hacia algún elemento de $X^{+} \cup X^{-} \cup Z^{-}$.

La figura 4.6 muestra este caso.

3. Finalmente, para una trayectoria con vértice final en $Z$ tenemos la siguiente.

Afirmación 4.5. Si $P$ es una trayectoria con vértice final un elemento $z^{\prime \prime} \in Z$, es decir, de la forma

$$
P_{6}: \underbrace{x^{\prime} \rightarrow y \rightarrow z \rightarrow x \rightarrow y^{\prime}}_{P_{2}} \rightarrow \cdots \rightarrow z^{\prime \prime}
$$




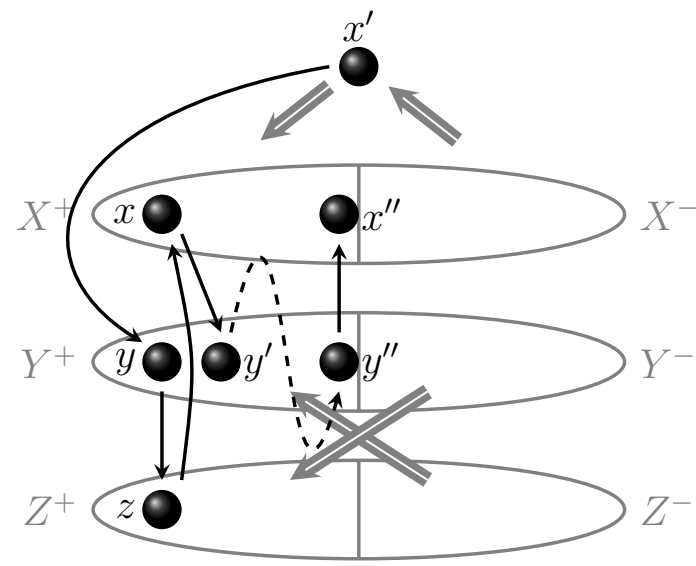

a) Trayectoria $P_{4}$.

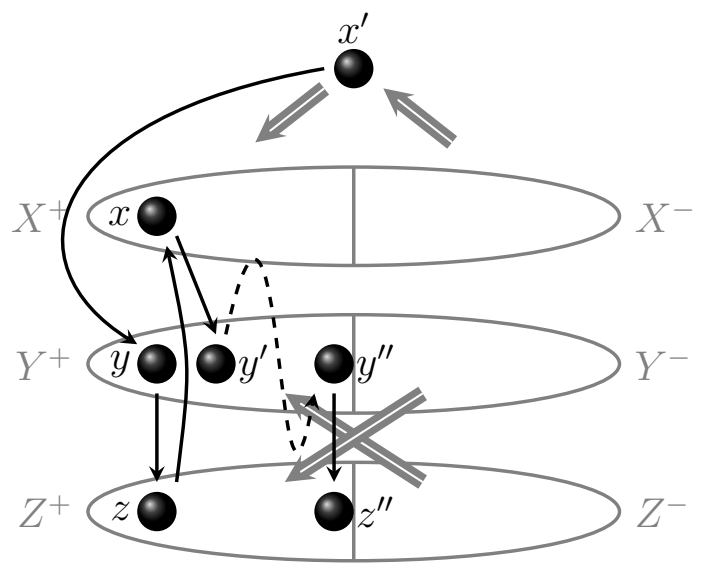

b) Trayectoria $P_{5}$.

Figura 4.6: Trayectorias de la afirmación 4.4.

o bien

$$
P_{7}: \underbrace{x^{\prime} \rightarrow y \rightarrow z \rightarrow x \rightarrow z^{\prime}}_{P_{3}} \rightarrow \cdots \rightarrow z^{\prime \prime}
$$

entonces existe $x^{\prime \prime} \in X$ o $y^{\prime \prime} \in Y^{+}$tal que $P$ puede ser extendida a una trayectoria de la forma

$$
P_{6}^{\prime}: \underbrace{x^{\prime} \rightarrow y \rightarrow z \rightarrow x \rightarrow y^{\prime} \rightarrow \cdots \rightarrow z^{\prime \prime}}_{P_{6}} \rightarrow x^{\prime \prime}
$$

o de la forma

$$
P_{7}^{\prime}: \underbrace{x^{\prime} \rightarrow y \rightarrow z \rightarrow x \rightarrow z^{\prime} \rightarrow \cdots \rightarrow z^{\prime \prime}}_{P_{7}} \rightarrow y^{\prime \prime}
$$

Demostración. Al igual que el caso anterior, notemos que $x^{\prime \prime} \neq u$ y $y^{\prime \prime} \neq u$ para toda $u \in V(P)$, pues si $x^{\prime \prime}$ o $z^{\prime \prime}$ coincidiera con alguna $u \in V(P)$, tendríamos un ciclo que pasa por tres de las partes y por el teorema 4.1 tenemos un $\overrightarrow{C_{3}}$ heterocromático. Notemos que $z^{\prime \prime} \notin Z^{-}$, pues en caso contrario, la trayectoria $P$ junto con la flecha $z^{\prime \prime} \rightarrow x^{\prime}$ formarían un ciclo que pasa por las tres clases cromáticas, una contradicción. Por lo tanto $z^{\prime \prime} \in Y^{+}$.

Supongamos que es imposible extender la trayectoria $P_{3}$ de $z^{\prime \prime}$ hacia algún elemento de $X \cup Y$, entonces $X \cup Y \Rightarrow z$ y $X \cup Y \subseteq N^{+}(z)$. Así $|X \cup Y| \leq n$.

Por otro lado,

$$
\begin{aligned}
N^{+}\left(N^{+}\left(z^{\prime \prime}\right)\right) \backslash N^{+}\left(z^{\prime \prime}\right) & =N^{+}\left(Z \backslash\left\{z^{\prime \prime}\right\}\right) \\
& \subseteq(X \cup Y) \backslash\{u\},
\end{aligned}
$$


donde $u \rightarrow z^{\prime \prime}$ y $u \in X \cup Y \cup V(P)$. Finalmente tenemos que

$$
\left|N^{+}\left(N^{+}\left(z^{\prime \prime}\right)\right) \backslash N^{+}\left(z^{\prime \prime}\right)\right|=|(X \cup Y) \backslash\{u\}| \leq n-1,
$$

en contradicción al corolario 4.3.

La figura 4.7 muestra este caso.

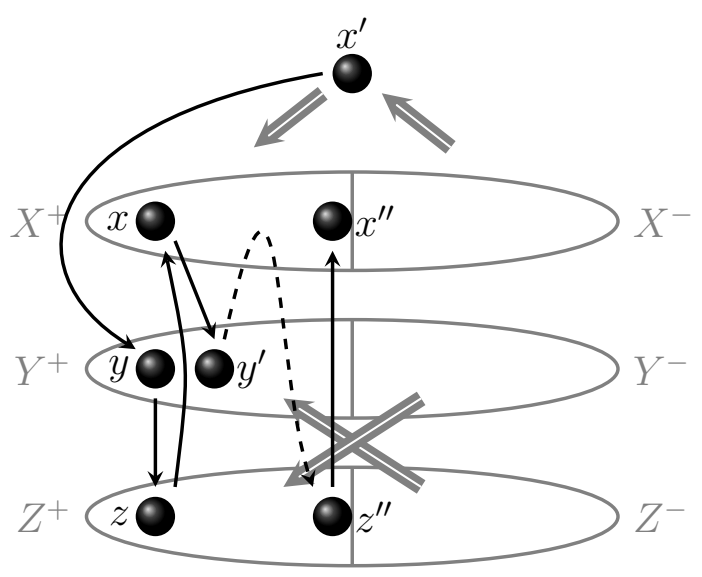

a) Trayectoria $P_{6}$.

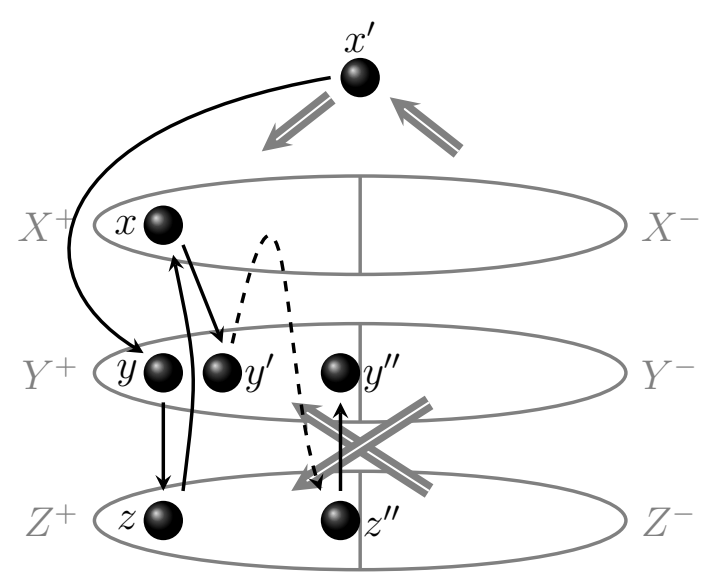

b) Trayectoria $P_{7}$.

Figura 4.7: Trayectorias de la afirmación 4.5.

Con todas la afirmaciones anteriores, podemos concluir que al considerar la clase cromática a la que pertenece el vértice final de la trayectoria $P$, siempre es posible extenderla.

Si continuamos este procedimiento y considerando que $V(T)$ es un conjunto finito, entonces en algún momento este proceso termina. Luego, no podemos extender las trayectorias y algún vértice se repite, esto señala que se forma un ciclo que pasa por las tres partes y por el teorema 4.1 se obtiene una contradicción. Lo anterior demuestra que

$$
N^{+}\left(N^{+}\left(N^{+}(X) \cap Y\right) \cap Z\right) \cap X=\emptyset .
$$

La prueba para el caso

$$
N^{+}\left(N^{+}\left(N^{+}(Y) \cap X\right) \cap Z\right) \cap Y=\emptyset
$$

se sigue de manera similar a la anterior. Si suponemos por contradicción que existe $y \in N^{+}\left(N^{+}\left(N^{+}(Y) \cap X\right) \cap Z\right) \cap Y$, entonces es posible construir una trayectoria

$$
P_{1}^{\prime}: y^{\prime} \rightarrow x \rightarrow z \rightarrow y
$$

y los argumentos para la demostración son análogos. 
El siguiente lema es complementario al anterior, ya que sus casos se pueden seguir de manera similar y la construcción de las trayectorias se toman en sentido inverso, es decir, extendemos las trayectoria agregando vértices al principio de la trayectoria.

Lema 4.3. Sean $T$ un torneo regular y $\varphi: V(T) \rightarrow\{X, Y, Z\}$ una coloración libre de $\overrightarrow{C_{3}}$ heterocromáticos, con $|X| \leq|Y| \leq|Z|$, entonces

$$
N^{-}\left(N^{-}\left(N^{-}(X) \cap Y\right) \cap Z\right) \cap X=\emptyset
$$

y

$$
N^{-}\left(N^{-}\left(N^{-}(Y) \cap X\right) \cap Z\right) \cap Y=\emptyset
$$

Demostración. Se sigue de manera análoga a la demostración del lema 4.2. Supongamos que existe $x \in N^{-}\left(N^{-}\left(N^{-}(X) \cap Y\right) \cap Z\right) \cap X$, entonces es posible construir una trayectoria

$$
P_{1}^{\prime \prime}: x \rightarrow z \rightarrow y \rightarrow x^{\prime}
$$

y los argumentos para extender una trayectoria $P$ con $P_{1}^{\prime \prime}$ como subtrayectoria son similares. De igual manera se prueba que

$$
N^{-}\left(N^{-}\left(N^{-}(Y) \cap X\right) \cap Z\right) \cap Y=\emptyset
$$

al suponer que la trayectoria

$$
P_{1}^{\prime \prime \prime}: y \rightarrow z \rightarrow x \rightarrow y^{\prime}
$$

existe y los argumentos son similares.

De la demostración de los lemas 4.2 y 4.3, podemos observar que existen ciertas trayectorias que son imposibles entre las clases cromáticas de un torneo $T$. Para dar esta propiedad, definamos lo siguiente.

Sea $\varphi: V(T) \rightarrow\left\{X_{1}, X_{2}, \ldots, X_{r}\right\}$ una $r$-coloración de los vértices del torneo $T$. Sea $\left\{s_{1}, s_{2}, \ldots, s_{k}\right\}$ una permutación de un subconjunto de $\{1,2, \ldots, r\}$ tal que $3 \leq k \leq r$. Denotemos por

$$
X_{s_{1}} \rightarrow X_{s_{2}} \rightarrow \ldots \rightarrow X_{s_{r}}
$$

al conjunto de trayectorias tal que

$$
x_{s_{1}} \rightarrow x_{s_{2}} \rightarrow \ldots \rightarrow x_{s_{r}}
$$

es una trayectoria en $T$, donde $x_{s_{i}} \in X_{s_{i}}$ para toda $i \in\{1,2, \ldots, r\}$.

Corolario 4.4. Sea $T$ un torneo regular no tenso tal que $\varphi: V(T) \rightarrow\{X, Y, Z\}$ es una 3-coloración libre de $\overrightarrow{C_{3}}$ heterocromáticos. Entonces las siguientes trayectorias son prohibidas en $T$.

$$
\begin{aligned}
& F P_{1}: \quad X \rightarrow Y \rightarrow Z \rightarrow X, \\
& F P_{2}: X \rightarrow Z \rightarrow Y \rightarrow X, \\
& F P_{3}: Y \rightarrow X \rightarrow Z \rightarrow Y, \\
& F P_{4}: Y \rightarrow Z \rightarrow X \rightarrow Y .
\end{aligned}
$$


Demostración. Notemos que en la demostración del lema 4.2, las trayectorias

$$
P_{1}: x \rightarrow y \rightarrow z \rightarrow x^{\prime} \quad \text { y } \quad P_{1}^{\prime}: y \rightarrow x \rightarrow z \rightarrow y^{\prime}
$$

no existen en $T$, por lo que las trayectorias

$$
F P_{1}: X \rightarrow Y \rightarrow Z \rightarrow X \quad \text { y } \quad F P_{3}: X \rightarrow Z \rightarrow Y \rightarrow X
$$

son prohibidas. De manera similar, por el lema 4.3, las trayectorias

$$
P_{1}^{\prime \prime}: x \rightarrow z \rightarrow y \rightarrow x^{\prime} \quad \text { y } \quad P_{1}^{\prime \prime \prime}: y \rightarrow z \rightarrow x \rightarrow y^{\prime}
$$

son imposibles, por lo que las trayectorias

$$
F P_{2}: Y \rightarrow X \rightarrow Z \rightarrow Y \quad \text { y } \quad F P_{4}: Y \rightarrow Z \rightarrow X \rightarrow Y
$$

son prohibidas.

Dada una coloración de los vértices de un torneo, si esta coloración tiene más de cuatro clases cromáticas y es libre de triángulos dirigidos heterocromáticos, entonces la unión de dos o más clases cromáticas, también nos proporciona una coloración libre de triángulos heterocromáticos. Esto lo damos en la siguiente:

Observación 4.1. Sean $T$ un torneo regular no tenso y $\varphi: V(T) \rightarrow\left\{X_{1}, X_{2}, \ldots, X_{r}\right\}$ una r-coloración libre de $\overrightarrow{C_{3}}$ heterocromáticos, tal que $1 \leq\left|X_{1}\right| \leq\left|X_{2}\right| \leq \cdots \leq\left|X_{r}\right|$. Sea $S=\left\{s_{1}, s_{2}, \ldots, s_{r}\right\}$ una permutación del conjunto $\{1,2, \ldots, r\}$. Si $r \geq 4$, entonces para toda $k<r$, la $k$-coloración $\psi: V(T) \rightarrow\left\{Y_{1}, Y_{2}, \ldots, Y_{k}\right\}$ definida por

$$
Y_{1}=\bigcup_{i=1}^{m_{1}} X_{s_{i}}, \quad Y_{2}=\bigcup_{i=m_{1}+1}^{m_{2}} X_{s_{i}}, \quad \cdots, \quad Y_{k}=\bigcup_{i=m_{k}+1}^{r} X_{s_{i}}
$$

donde $1 \leq m_{1}<m_{2}<\cdots<m_{k} \leq r-1$, es libre de $\overrightarrow{C_{3}}$ heterocromáticos (por la definición de inconexión acíclica). En particular, haremos uso de la 3-coloración $\psi: V(T) \rightarrow\{X, Y, Z\}$ definida por

$$
X=\bigcup_{i=1}^{m} X_{s_{i}}, \quad Y=\bigcup_{i=m+1}^{n} X_{s_{i}} \quad y \quad Z=\bigcup_{i=n+1}^{r} X_{s_{i}}
$$

donde $1 \leq m<n \leq r-1$.

Observación 4.2. Sean $T$ un torneo regular no tenso y $\varphi: V(T) \rightarrow\left\{X_{1}, X_{2}, \ldots, X_{r}\right\}$ una $r$-coloración óptima libre de $\overrightarrow{C_{3}}$ heterocromáticos, con $r \geq 4$. Sea una 3-coloración $\psi: V(T) \rightarrow\{X, Y, Z\}$ como en la observación 4.1 .

Sin pérdida de generalidad supongamos que $|X| \leq|Y| \leq|Z|$. Así, de los lemas 4.2 y 4.3 podemos concluir que el torneo tripartito

$$
M=T \backslash(A(T\langle X\rangle) \cup A(T\langle Y\rangle) \cup A(T\langle Z\rangle))
$$

es libre de trayectorias de los tipos $F P_{1}, F P_{2}, F P_{3}$ y $F P_{4}$ del corolario 4.4. 
Sean el torneo circulante $\vec{C}_{2 n+1}(J)$ y $\varphi: V\left(\vec{C}_{2 n+1}(J)\right) \rightarrow\{X, Y, Z\}$ una 3-coloración libre de $\vec{C}_{3}$ heterocromáticos. Es claro que $V\left(\vec{C}_{2 n+1}(J)\right)=X|Y| Z$ nos proporciona una partición y recordemos que en el lenguaje de la teoría aditiva de los números, dado un conjunto $S \subset V\left(\vec{C}_{2 n+1}(J)\right)$, se tiene que $N^{+}(S)=S+J$.

El lema $4.2^{2}$ es la generalización del siguiente lema dado por B. Llano y V. NeumannLara en [LINL07]:

Corolario 4.5 (Lema 1 en [LlNL07]). Sea $P=X|Y| Z$ una partición externamente libre de $\overrightarrow{C_{3}}$ de los vértices de $\vec{C}_{2 n+1}(J)$. Entonces

$$
((((X+J) \cap Y)+J) \cap Z)+J) \cap Y=\emptyset .
$$

Demostración. Se sigue por el lema 4.2.

Recordemos que si un torneo regular tiene como orden un entero primo, entonces es primo. En [LlNL07], B. Llano y V. Neumann-Lara muestran con la ayuda del corolario anterior, que todo torneo circulante de orden un entero primo, es tenso. Con la misma idea, demostramos en la siguiente sección que los torneos regulares primos de cualquier orden son tensos.

\subsection{Torneos tensos y no tensos}

Dado un torneo regular, los lemas 4.2 y 4.3 nos restringen a considerar una 3-coloración de los vértices de $T$, por lo que la pregunta natural es

¿Qué sucede en un torneo regular $T$ con $\overrightarrow{\omega_{3}}(T) \geq 4$ ?

En esta sección hacemos uso de dichos lemas para demostrar una generalización de los mismos y damos una caracterización para los torneos regulares tensos.

El siguiente teorema es consecuencia de los lemas antes mencionados y es la demostración de la conjetura $3.2^{3}$ mencionada al principio del capítulo.

Teorema 4.2 (Demostración de la conjetura 3.2). Todo torneo regular $T$ es primo si y solo si es tenso.

Demostración. Demostremos la contrapositiva de la necesidad, es decir, demostremos que si $T$ es un torneo regular no tenso, entonces es una suma de Zykov.

Supongamos que $T$ es un torneo regular no tenso, tal que $\overrightarrow{\omega_{3}}(T)=r \geq 3$. Entonces existe una $r$-coloración $\varphi: V(T) \rightarrow\left\{X_{1}, X_{2}, \ldots, X_{r}\right\}$ libre de $\overrightarrow{C_{3}}$ heterocromáticos. Por la observación 4.1, a partir de esta $r$-coloración, podemos definir una 3-coloración

\footnotetext{
${ }^{2}$ página 54

${ }^{3}$ página 50
} 
$\psi: V(T) \rightarrow\{X, Y, Z\}$ que es libre de $\overrightarrow{C_{3}}$ heterocromáticos. Supongamos sin pérdida de generalidad que $|X| \leq|Y| \leq|Z|$ y sea $x \in X$. Definimos la partición

$$
V(T)=\{x\}\left|X^{+}\right| X^{-}\left|Y^{+}\right| Y^{-}\left|Z^{+}\right| Z^{-}
$$

como en la demostración del lema 4.2.

Dado que la coloración $\varphi$ es libre de $\overrightarrow{C_{3}}$ heterocromáticos, entonces $Y^{-} \Rightarrow Z^{+}$, pues en caso contrario si existieran $y_{2} \in Y^{-}$y $z_{1} \in Z^{+}$tales que $z_{1} \rightarrow y_{2}$, entonces

$$
\overrightarrow{C_{3}}: x \rightarrow z_{1} \rightarrow y_{2} \rightarrow x
$$

sería heterocromático. Similarmente, $Z^{-} \Rightarrow Y^{+}$ya que en caso contrario si existieran $y_{1} \in Y^{+}$y $z_{2} \in Z^{-}$tales que $y_{1} \rightarrow z_{2}$, entonces

$$
\overrightarrow{C_{3}}: x \rightarrow y_{1} \rightarrow z_{2} \rightarrow x
$$

sería heterocromático.

Notemos que si $|X|=1$, entonces $X^{+}=X^{-}=\emptyset$, por lo tanto no hay nada que probar pues $\{x\} \cup Y$ definen una componente para una suma de Zykov no trivial, donde la base $B$ se obtiene al aplicar el epimorfismo $\pi: V(T) \rightarrow V(B)$, al conjunto $\{x\} \cup Y$ y así la suma de Zykov es

$$
B\left[\left\{T\langle\{x\} \cup Y\rangle,|Z| \cdot K_{1}\right\}\right]
$$

Supongamos que $|X| \geq 2$, por lo que $X^{+} \neq \emptyset$ o $X^{-} \neq \emptyset$. Observe los siguientes casos que se siguen por el corolario $4.4^{4}$.

(i) $Y^{+} \Rightarrow Z^{+}$, en caso contrario, si existieran $z_{1} \in Z^{+}$y $y_{1} \in Y^{+}$tales que $z_{1} \rightarrow y_{1}$, entonces $y_{2} \rightarrow x \rightarrow z_{1} \rightarrow y_{1}$ sería una trayectoria prohibida

$$
F P_{3}: Y \rightarrow X \rightarrow Z \rightarrow Y .
$$

(ii) Similarmente, $Z^{-} \Rightarrow Y^{-}$, en caso contrario, si existieran $y_{2} \in Y^{-}$y $z_{2} \in Z^{-}$tales que $y_{2} \rightarrow z_{2}$, entonces $y_{2} \rightarrow z_{2} \rightarrow x \rightarrow y_{1}$ sería una trayectoria prohibida

$$
F P_{4}: Y \rightarrow Z \rightarrow X \rightarrow Y \text {. }
$$

(iii) $X^{+} \Rightarrow Z^{+}$, en caso contrario, si existieran $z_{1} \in Z^{+}$y $x_{1} \in X^{+}$tales que $z_{1} \rightarrow x_{1}$, entonces $x \rightarrow y_{1} \rightarrow z_{1} \rightarrow x_{1}$ sería una trayectoria prohibida

$$
F P_{1}: X \rightarrow Y \rightarrow Z \rightarrow X
$$

(iv) $Z^{-} \Rightarrow X^{-}$, en caso contrario, si existieran $x_{2} \in X^{-}$y $z_{2} \in Z^{-}$tales que $y_{2} \rightarrow z_{2}$, entonces por el inciso (ii), tenemos que para toda $z_{2} \in Z^{-}$y $y_{2} \in Z^{-}$, se tiene que $x_{2} \rightarrow z_{2} \rightarrow y_{2} \rightarrow x$ sería una trayectoria prohibida

$$
F P_{2}: X \rightarrow Z \rightarrow Y \rightarrow X
$$

${ }^{4}$ página 62 

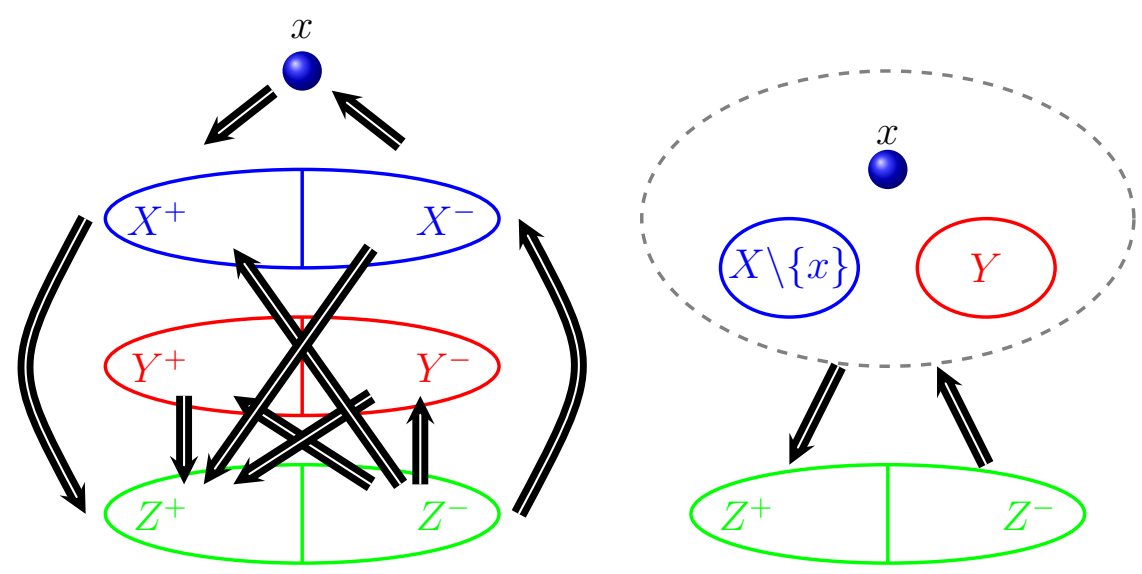

Figura 4.8: El torneo no tenso $T$ es una suma de Zykov.

(v) $Z^{-} \Rightarrow X^{+}$, en caso contrario, si existieran $x_{1} \in X^{+}$y $z_{2} \in Z^{-}$tales que $x_{1} \rightarrow z_{2}$, entonces por el inciso (ii), tenemos que para toda $z_{2} \in Z^{-}$y $y_{2} \in Z^{-}$, se tiene que $x_{1} \rightarrow z_{2} \rightarrow y_{2} \rightarrow x$ sería una trayectoria prohibida

$$
F P_{2}: X \rightarrow Z \rightarrow Y \rightarrow X
$$

(vi) $X^{-} \Rightarrow Z^{+}$, en caso contrario, si existieran $x_{1} \in X^{-}$y $z_{1} \in Z^{+}$tales que $z_{1} \rightarrow x_{2}$, entonces $x \rightarrow y_{1} \rightarrow z_{1} \rightarrow x_{2}$ sería una trayectoria prohibida

$$
F P_{1}: X \rightarrow Z \rightarrow Y \rightarrow X
$$

En la figura 4.8 podemos observar que, como consecuencia de los puntos anteriores, el torneo $T$ tiene la estructura de una suma de Zykov no trivial, cuya base $B$ es el torneo que se obtiene al aplicar la proyección natural $\pi: V(T) \rightarrow V(B)$ al conjunto $X \cup Y$ en los vértices de $T$, es decir, la base $B$ tiene asociado el vértice $\pi(X \cup Y)$ con el conjunto $X \cup Y$.

Por el lema 1.3, tenemos que $T\langle X \cup Y\rangle$ es un torneo regular. Así la familia de torneos regulares $\alpha$, está formada por el torneo $T\langle X \cup Y\rangle$ y $|Z|$ veces un vértice singular, es decir

$$
\alpha=\left\{T\langle X \cup Y\rangle,|Z| \cdot K_{1}\right\}
$$

y la suma de Zykov es

$$
B\left[\left\{T\langle X \cup Y\rangle,|Z| \cdot K_{1}\right\}\right]
$$

La suficiencia se sigue por la contrapositiva del corolario $1.2^{5}$, ya que si $T$ no es tenso, entonces no es primo. Así finalmente tenemos que $T$ es primo si y solo si es tenso.

${ }^{5}$ página 12 
Notemos que el teorema anterior hace uso del hecho de que la observación 4.1, nos proporciona una manera de definir una 3-coloración a partir de una $r$-coloración $(r \geq 4)$. Lo anterior lo generalizamos en el teorema 4.4 que aparece más adelante, pero debido a la complejidad que representa para el lector el seguimiento de la demostración de dicho teorema, describimos el caso para una 4-coloración.

Teorema 4.3. Sea $T$ un torneo regular tal que $\overrightarrow{\omega_{3}}(T)=4$, entonces $T$ es una suma de Zykov no trivial.

Demostración. Sea

$$
\varphi: V(T) \rightarrow\left\{X_{1}, X_{2}, X_{3}, X_{4}\right\}
$$

una 4-coloración libre de $\overrightarrow{C_{3}}$ heterocromáticos. Supongamos sin pérdida de generalidad que $\left|X_{1}\right| \leq\left|X_{2}\right| \leq\left|X_{3}\right| \leq\left|X_{4}\right|$.

Sea $x \in X_{1}$ y definimos la partición de $V(T)$

$$
P_{1}=\{x\}\left|X_{1}^{+}\right| X_{1}^{-}\left|X_{2}^{+}\right| X_{2}^{-}\left|X_{3}^{+}\right| X_{3}^{-}\left|X_{4}^{+}\right| X_{4}^{-}
$$

como en la demostración del lema 4.2. Definamos a partir de $P_{1}$ a la partición $P_{2}$ de $V(T)$ como

$$
P_{2}=\{x\}\left|X_{1}^{+}\right| X_{1}^{-}\left|X_{2}^{+}\right| X_{2}^{-}\left|X_{3}^{+} \cup X_{4}^{+}\right| X_{3}^{-} \cup X_{4}^{-}
$$

Por la observación $4.1^{6}$ tenemos que la partición $P_{2}$ proporciona una 3 -coloración libre de $\overrightarrow{C_{3}}$ heterocromáticos. Por otra parte, observemos en la figura 4.9 que por el teorema $4.2^{7}$, tenemos que

$$
X_{3}^{-} \cup X_{4}^{-} \Rightarrow X_{1} \cup X_{2} \quad \text { y } \quad X_{1} \cup X_{2} \Rightarrow X_{3}^{+} \cup X_{4}^{+}
$$

Notemos que solo nos resta determinar las direcciones de las flechas entre $X_{3}^{+}$y $X_{4}^{+}$, y entre $X_{3}^{-}$y $X_{4}^{-}$. Sea

$$
P_{3}=\{x\}\left|X_{1}^{+} \cup X_{2}^{+}\right| X_{1}^{-} \cup X_{2}^{-}\left|X_{3}^{+}\right| X_{3}^{-}\left|X_{4}^{+}\right| X_{4}^{-}
$$

una partición similar a la anterior y observemos que

$$
\left|X_{1} \cup X_{2}\right| \leq\left|X_{3}\right| \leq\left|X_{4}\right| \quad \text { o } \quad\left|X_{3}\right| \leq\left|X_{1} \cup X_{2}\right| \leq\left|X_{4}\right|
$$

ya que en otro caso $\left|X_{3}\right| \leq\left|X_{4}\right| \leq\left|X_{1} \cup X_{2}\right|$ es imposible, debido a que como $X_{4}^{-} \Rightarrow X_{3}^{-}$, $X_{3}^{-} \Rightarrow X_{1}^{-} \cup X_{2}^{-}$y $X_{1}^{-} \cup X_{2}^{-} \Rightarrow X_{4}^{+}$, entonces se inducen trayectorias del tipo

$$
F P_{4}: X_{4} \rightarrow X_{3} \rightarrow\left(X_{1} \cup X_{2}\right) \rightarrow X_{4}
$$

que por el corolario 4.4, son imposibles.

Nuevamente aplicando el teorema 4.2 , tenemos que $X_{3}^{+} \Rightarrow X_{4}^{+}$y $X_{3}^{-} \Leftarrow X_{4}^{-}$, por lo que tiene la estructura de una suma de Zykov no trivial, donde la base $B$ se obtiene al

\footnotetext{
${ }^{6}$ página 63

${ }^{7}$ página 64
} 

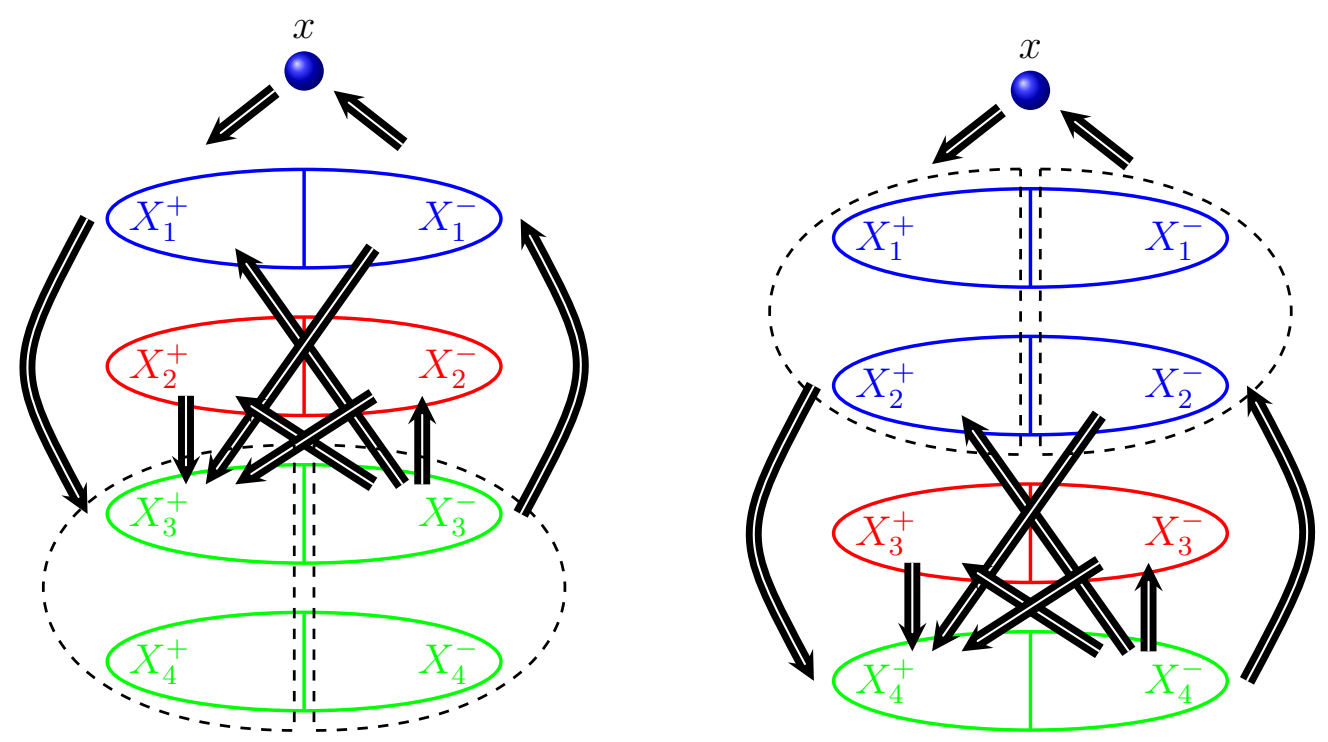

Figura 4.9: El torneo regular con cuatro clases cromáticas.

aplicar el epimorfismo $\pi: V(T) \rightarrow V(B)$ al conjunto $X_{1} \cup X_{2}$, es decir, la base $B$ tiene asociado el vértice $\pi\left(X_{1} \cup X_{2}\right)$ al conjunto $X_{1} \cup X_{2}$. Por lo tanto la familia no trivial $\alpha=\left\{T\left\langle X_{1} \cup X_{2}\right\rangle,\left(\left|X_{3}\right|+\left|X_{4}\right|\right) \cdot K_{1}\right\}$ y así, la suma de Zykov resultante es

$$
B\left[\left\{T\left\langle X_{1} \cup X_{2}\right\rangle,\left(\left|X_{3}\right|+\left|X_{4}\right|\right) \cdot K_{1}\right\}\right]
$$

En la figura 4.9 se puede observar los casos de esta demostración.

Como consecuencia de los lemas 4.2 y 4.3, tenemos el corolario $4.4^{8}$, que es una síntesis de las trayectorias prohibidas en un torneo regular no tenso $T$. Notemos en el teorema 4.2, que dada una 3-coloración $\varphi: V(T) \rightarrow\left\{X_{1}, X_{2}, X_{3}\right\}$ libre de $\overrightarrow{C_{3}}$ heterocromáticos, tal que $\left|X_{1}\right| \leq\left|X_{2}\right| \leq\left|X_{3}\right|$, entonces las trayectorias prohibidas de longitud al menos cuatro entre las tres clases cromáticas, son aquellas que comienzan y terminan en $X_{1}$ o $X_{2}$.

Similarmente, en el teorema 4.3, dada una 4-coloración $\varphi: V(T) \rightarrow\left\{X_{1}, X_{2}, X_{3}, X_{4}\right\}$, tal que $\left|X_{1}\right| \leq\left|X_{2}\right| \leq\left|X_{3}\right| \leq\left|X_{4}\right|$, entonces las trayectorias prohibidas de longitud cuatro entre tres clases cromáticas, son aquellas que, si pasan por la clase cromática de mayor cardinalidad, entonces es imposible que dichas trayectorias se extiendan a alguna clase cromática de menor cardinalidad.

Lo anterior lo resumimos en el siguiente teorema, que es una generalización de los lemas 4.2 y 4.3 .

${ }^{8}$ página 62 
Teorema 4.4. Sean $T$ un torneo regular no tenso y $\varphi: V(T) \rightarrow\left\{X_{1}, X_{2}, \ldots, X_{r}\right\}$ una $r$-coloración libre de $\overrightarrow{C_{3}}$ heterocromáticos $(r \geq 3)$. Supongamos sin pérdida de generalidad que $\left|X_{1}\right| \leq\left|X_{2}\right| \leq \cdots \leq\left|X_{r}\right|$. Entonces

$$
N^{+}\left(N^{+}\left(N^{+}\left(X_{i}\right) \cap X_{j}\right) \cap X_{k}\right) \cap X_{i}=\emptyset
$$

y

$$
N^{-}\left(N^{-}\left(N^{-}\left(X_{i}\right) \cap X_{j}\right) \cap X_{k}\right) \cap X_{i}=\emptyset,
$$

donde $i, j, k \in\{1,2, \ldots, r\}$, tal que $i, j, k$ son distintos y $k=\operatorname{máx}\{i, j, k\}$.

Demostración. La prueba es por inducción sobre el número de colores $r$. Sea

$$
\varphi: V(T) \rightarrow\left\{X_{1}, X_{2}, \ldots, X_{r}\right\}
$$

una $r$-coloración libre de $\overrightarrow{C_{3}}$ heterocromáticos $(r \geq 3)$.

El caso base $r=3$ se sigue a partir de la observación 4.1, al definir una 3-coloración a partir de la $r$-coloración original y por el lema 4.2 se tiene lo deseado.

Supongamos que $r \geq 4$ y que el teorema se cumple para toda $(r-1)$-coloración libre de $\overrightarrow{C_{3}}$ heterocromáticos. Sea $x \in X_{1}$ y definamos para toda $i \in\{1,2, \ldots, r\}$ a $X_{i}^{+}=N^{+}(x) \cap X_{i}$ y a $X_{i}^{-}=N^{-}(x) \cap X_{i}$ como en la figura 4.10. Obtenemos la partición

$$
P_{1}=\{x\}\left|X_{1}^{+}\right| X_{2}^{+}|\ldots| X_{r}^{+}\left|X_{1}^{-}\right| X_{2}^{-}|\ldots| X_{r}^{-} .
$$

Como $\varphi: V(T) \rightarrow\left\{X_{1}, X_{2}, \ldots, X_{r}\right\}$ es libre de $\overrightarrow{C_{3}}$ heterocromáticos, se tiene que

$$
X_{j}^{-} \Rightarrow X_{i}^{+} \quad \mathrm{y} \quad X_{i}^{-} \Rightarrow X_{j}^{+}
$$

para $2 \leq i<j \leq r-1$ como se muestra en la figura 4.10. Sea la partición

$$
P_{2}=\{x\}\left|Y_{1}^{+}\right| Y_{2}^{+}|\ldots| Y_{r-1}^{+}\left|Y_{1}^{-}\right| Y_{2}^{-}|\ldots| Y_{r-1}^{-}
$$

definida por

$$
Y_{i}^{+}= \begin{cases}X_{i}^{+}, & \text {si } i \in\{1,2, \ldots, r-2\} \\ X_{r-1}^{+} \cup X_{r}^{+}, & \text {si } i=r-1,\end{cases}
$$

y similarmente

$$
Y_{i}^{-}= \begin{cases}X_{i}^{-}, & \text {si } i \in\{1,2, \ldots, r-2\}, \\ X_{r-1}^{-} \cup X_{r}^{-}, & \text {si } i=r-1 .\end{cases}
$$

En la figura 4.11 podemos observar la partición $P_{2}$ definida a partir de la partición $P_{1}$. Por la observación 4.1 , tenemos que

$$
\phi: V(T) \rightarrow\left\{Y_{1}, Y_{2}, \ldots, Y_{r-1}\right\}
$$

es una $(r-1)$-coloración libre de $\overrightarrow{C_{3}}$ heterocromáticos y similarmente a la coloración $\varphi$, tenemos que $Y_{i}^{-} \Rightarrow Y_{j}^{+}$para toda $i, j \in\{1,2, \ldots, r-1\}$, con $i \neq j$. Además $\left|Y_{i}\right| \leq\left|Y_{i+1}\right|$ para toda $i \in\{1,2, \ldots, r-2\}$. 


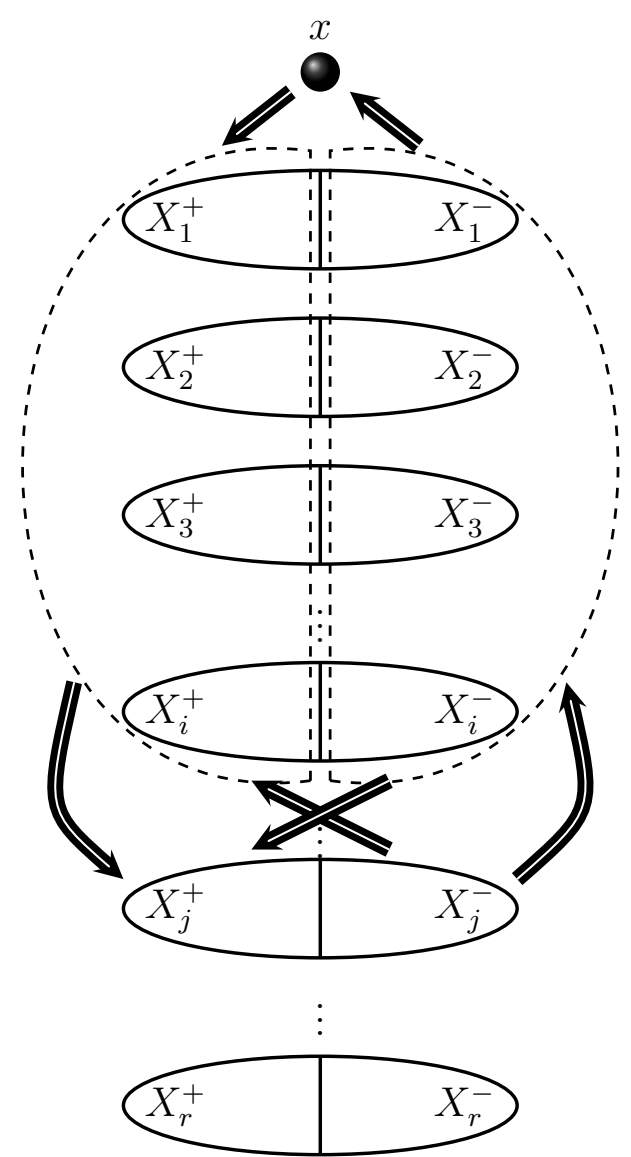

Figura 4.10: El torneo $T$ y la partición con respecto a $x$.

Al aplicar la hipótesis de inducción, tenemos que $Y_{i}^{+} \Rightarrow Y_{j}^{+}$para $2 \leq i<j \leq r-1$. En caso contrario, si existieran $w_{j} \in Y_{j}^{+}$y $w_{i} \in Y_{i}^{+}$tales que $w_{j} \rightarrow w_{i}$, entonces dada $v_{i} \in Y_{i}^{-}$, la trayectoria $v_{i} \rightarrow u \rightarrow w_{j} \rightarrow w_{i}$ pasa por tres de las partes, que es una contradicción a la hipótesis de inducción.

De manera similar, podemos demostrar que $Y_{j}^{-} \Rightarrow Y_{i}^{-}$para toda $2 \leq i<j \leq r-1$, en caso contario, si existieran $w_{i} \in Y_{i}^{-}$y $w_{j} \in Y_{j}^{-}$tales que $w_{i} \rightarrow w_{j}$, entonces dada $v_{i} \in Y_{i}^{+}$tenemos que la trayectoria $w_{i} \rightarrow w_{j} \rightarrow u \rightarrow v_{i}$ pasa por tres de las partes, que es una contradicción a la hipótesis de inducción.

Notemos que si $\left|X_{1}\right|=1$, entonces $X_{1}^{+}=X_{1}^{-}=Y_{1}^{+}=Y_{1}^{-}=\emptyset$, ya que $x \in X_{1} \mathrm{y}$ $X_{1}^{+} \cup X_{1}^{-}=X \backslash\{x\}$. En otro caso, si $\left|X_{1}\right| \geq 2$, entonces se tiene que $X_{1}^{+} \neq \emptyset$ o $X_{1}^{-} \neq \emptyset$ o ambos. Tenemos los siguientes casos:

- Si $X_{1}^{+} \neq \emptyset$, entonces $Y_{1}^{+} \neq \emptyset$ y así $Y_{i}^{-} \Rightarrow Y_{1}^{+}$para toda $i \in\{3,4, \ldots, r-1\}$, en caso contrario si existieran $u_{1} \in Y_{1}^{+}$y $v \in Y_{i}^{-}$tales que $u_{1} \rightarrow v$, tendríamos que $u_{1} \rightarrow u \rightarrow w \rightarrow u$ es una trayectoria que pasa por tres de las partes para toda $w \in Y_{2}^{-}$, que es una contradicción a la hipótesis de inducción.

Mas aún, notemos que $Y_{1}^{+} \Rightarrow Y_{i}^{+}$para toda $i \in\{3,4, \ldots, r-1\}$, en caso contrario 
si existieran $u_{1} \in Y_{1}^{+}$y $v \in Y_{i}^{+}$tales que $v \rightarrow u_{1}$, tendríamos que para toda $w \in Y_{j}^{+}$con $2 \leq j<i \leq r-1$, la trayectoria $u \rightarrow w \rightarrow v \rightarrow u_{1}$ pasa por tres de las partes. Esto es una contradicción similar a la anterior.

- Si $X_{1}^{-} \neq \emptyset$, entonces $Y_{1}^{-} \neq \emptyset$ y $Y_{1}^{-} \Rightarrow Y_{i}^{+}$para toda $i \in\{3,4, \ldots, r-1\}$, en caso contrario, si existieran $u_{1} \in Y_{1}^{-}$y $v \in Y_{i}^{+}$tales que $v \rightarrow u_{1}$, tendríamos que la trayectoria $u \rightarrow w \rightarrow v \rightarrow u_{1}$, pasa por tres de las partes para toda $w \in X_{2}^{+}$, que es una contradicción a la hipótesis de inducción.

Así, $Y_{i}^{-} \Rightarrow Y_{1}^{-}$para $3 \leq i \leq r-1$, en caso contrario si existieran $u_{1} \in Y_{1}^{-}$y $v \in Y_{i}^{+}$tales que $u_{1} \rightarrow v$, tedríamos que $u_{1} \rightarrow v \rightarrow w \rightarrow u$ es una trayectoria que pasa por tres de las partes, para toda $w \in Y_{j}^{-}$, con $2 \leq i<j \leq r-1$.

Esto demuestra que para la $(r-1)$-coloración $\phi$ definida en (4.4), tenemos que

$$
N^{+}\left(Y_{1}^{+} \cup Y_{2}^{+}\right) \subset \bigcup_{i=3}^{r-1} Y_{i}^{+} \quad \text { y } \quad N^{-}\left(Y_{1}^{-} \cup Y_{2}^{-}\right) \subset \bigcup_{i=3}^{r-1} Y_{i}^{-},
$$

lo cual implica que, para la $r$-coloración original $\varphi$ definida en (4.2),

$$
N^{+}\left(X_{1}^{+} \cup X_{2}^{+}\right) \subset \bigcup_{i=3}^{r} X_{i}^{+} \quad \text { y } \quad N^{-}\left(X_{1}^{-} \cup X_{2}^{-}\right) \subset \bigcup_{i=3}^{r} X_{i}^{-}
$$

De (4.3) y (4.5) obtenemos que $X_{j}^{-} \Rightarrow X_{i}$ y $X_{j}^{+} \Leftarrow X_{j}$ para $2 \leq i<j \leq r$. Sin embargo, no podemos decir nada aún sobre la orientación de las flechas entre $X_{1}$ y $X_{2}$. Solo nos restaría determinar el comportamiento de las flechas entre $X_{r-1}^{+}$y $X_{r}^{+}$, así como aquellas entre $X_{r-1}^{-}$y $X_{r}^{-}$.

Sea la partición

$$
P_{3}=\{x\}\left|Z_{1}^{+}\right| Z_{2}^{+}|\ldots| Z_{r-1}^{+}\left|Z_{1}^{-}\right| Z_{2}^{-}|\ldots| Z_{r-1}^{-}
$$

de la figura 4.11, la cual se obtiene a partir de la partición $P_{1}$ al definir

$$
Z_{i}^{+}= \begin{cases}X_{1}^{+} \cup X_{2}^{+}, & \text {si } i=1, \\ X_{i+1}^{+}, & \text {si } i \in\{2,3, \ldots, r-1\}\end{cases}
$$

$\mathrm{y}$

$$
Z_{i}^{-}= \begin{cases}X_{1}^{-} \cup X_{2}^{+}, & \text {si } i=1, \\ X_{i+1}^{-}, & \text {si } i \in\{2,3, \ldots, r-1\} .\end{cases}
$$

Así, (4.5) se puede reescribir como

$$
N^{+}\left(Z_{1}^{+}\right) \subset \bigcup_{i=2}^{r-1} Z_{i}^{+} \quad \text { y } \quad N^{-}\left(Z_{1}^{-}\right) \subset \bigcup_{i=2}^{r-1} Z_{i}^{-} .
$$

La función

$$
\psi: V(T) \rightarrow\left\{Z_{1}, Z_{2}, \ldots, Z_{r-1}\right\}
$$



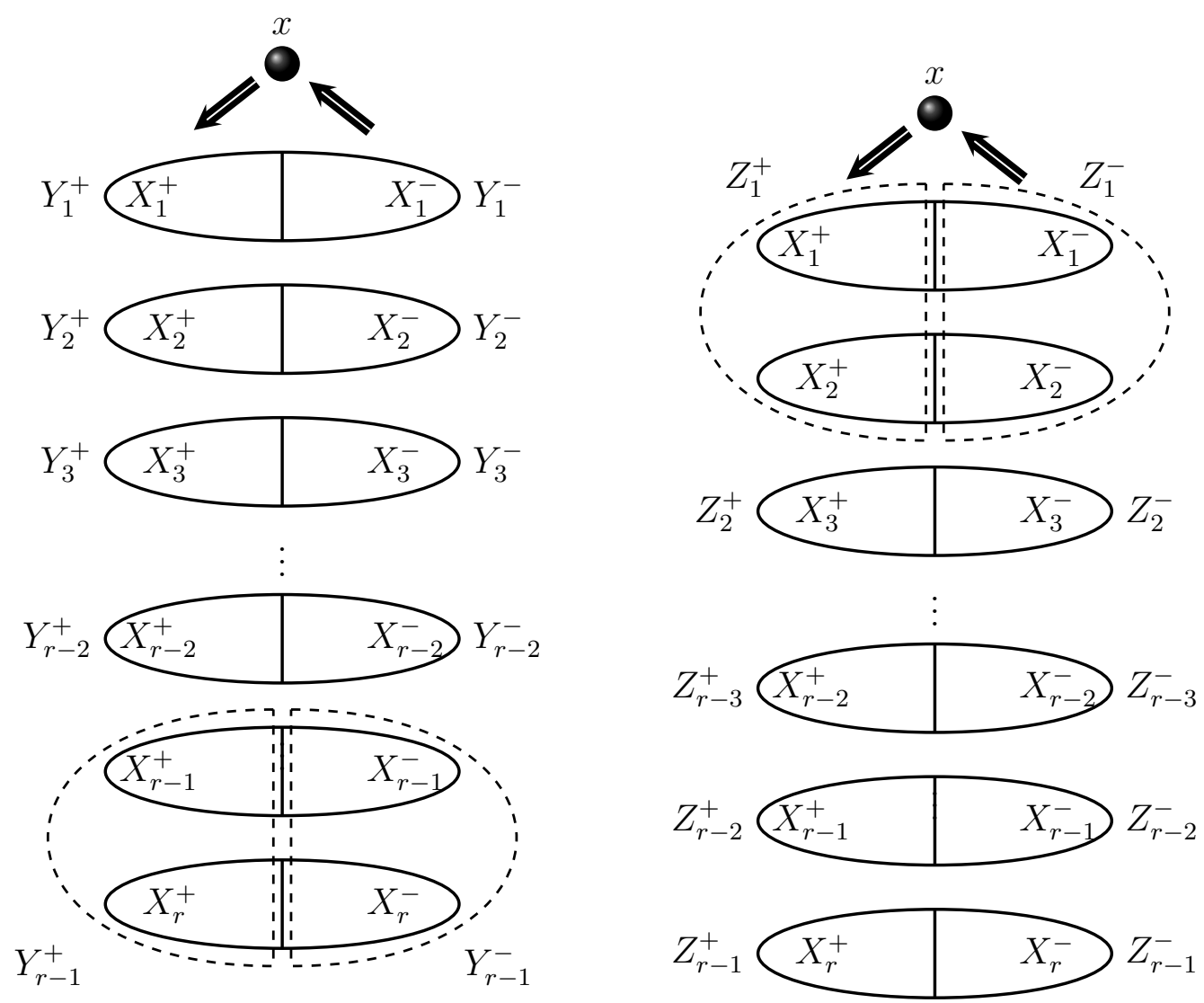

Figura 4.11: El torneo $T$ y las particiones $P_{2}$ y $P_{3}$ con respecto a $x$.

es una $(r-1)$-coloración libre de $\overrightarrow{C_{3}}$ heterocromáticos y similarmente al caso anterior con la coloración $\phi$, tenemos que $\left|Z_{i}\right| \leq\left|Z_{i+1}\right|$ para $i \in\{2,3, \ldots, r-2\}$.

Por la hipótesis de inducción llegamos a que $Z_{r-1}^{+} \Rightarrow Z_{r}^{+}$, ya que en caso contrario si existieran $w_{r} \in Z_{r}^{+}$y $w_{r-1} \in Z_{r-1}^{+}$tales que $w_{k} \rightarrow w_{r-1}$, tenemos que existe la trayectoria $v \rightarrow u \rightarrow w_{r} \rightarrow w_{r-1}$ para toda $v \in Z_{r-1}^{-}$, una contradicción. También se tiene que $Z_{r}^{-} \Rightarrow Z_{r-1}^{-}$, en otro caso si existieran $w_{r-1} \in Z_{r-1}^{-}$y $w_{r} \in Z_{r}^{-}$tales que $w_{r-1} \rightarrow w_{r}$, entonces tenemos la trayectoria $w_{r-1} \rightarrow w_{r} \rightarrow u \rightarrow v$ para toda $v \in Z_{r-1}^{+}$, una contradicción.

Por todo lo anterior, concluimos que en la partición original $P_{1}$ de los vértices, tenemos que si $\left|X_{1}\right| \leq\left|X_{2}\right| \leq \cdots \leq\left|X_{r}\right|$, entonces

$$
\begin{gathered}
X_{1}=X_{1}^{+} \cup X_{1}^{-} \Rightarrow \bigcup_{j=3}^{r} X_{j}^{+}, \quad X_{1}=X_{1}^{+} \cup X_{1}^{-} \Leftarrow \bigcup_{j=3}^{r} X_{j}^{-}, \\
X_{2}^{+} \Leftarrow \bigcup_{j=3}^{r} X_{j}^{-}, \quad X_{2}^{-} \Rightarrow \bigcup_{j=3}^{r} X_{j}^{+} .
\end{gathered}
$$


Para toda $i \in\{2,3, \ldots, r-1\}$

$$
X_{i}^{+} \Rightarrow \bigcup_{j=i+1}^{r} X_{j}^{+}, \quad X_{i}^{-} \Leftarrow \bigcup_{j=i+1}^{r} X_{j}^{-} .
$$

Finalmente para $i \in\{3,4, \ldots, r-1\}$

$$
X_{i}^{+} \Leftarrow \bigcup_{\substack{j=1 \\ i \neq j}}^{r} X_{j}^{-}, \quad X_{i}^{-} \Rightarrow \bigcup_{\substack{j=1 \\ i \neq j}}^{r} X_{j}^{+}
$$

Esta configuración carece de trayectorias de la forma $u \rightarrow v \rightarrow w \rightarrow u^{\prime}$ y de la forma $u^{\prime} \rightarrow w \rightarrow v \rightarrow u$, donde $\left\{u, u^{\prime}\right\} \subset X_{i}, v \in X_{j}$ y $w^{\prime} \in X_{k}$, todos estos vértices en particiones distintas, con $i, j<k$, por lo que

$$
N^{+}\left(N^{+}\left(N^{+}\left(X_{i}\right) \cap X_{j}\right) \cap X_{k}\right) \cap X_{i}=\emptyset
$$

y

$$
N^{-}\left(N^{-}\left(N^{-}\left(X_{i}\right) \cap X_{j}\right) \cap X_{k}\right) \cap X_{i}=\emptyset
$$

lo que demuestra el teorema.

Consideramos que el siguiente teorema es el más importante del presente capítulo, ya que nos permite dar una caracterización para los torneos regulares tensos y es una consecuencia del lema anterior. Notemos que es una generalización de los teoremas 4.2 y 4.3 para la inconexión libre de $\overrightarrow{C_{3}}$ en general.

Teorema 4.5. Sea $T$ un torneo regular con $\overrightarrow{\omega_{3}}(T)=r$. Si $T$ no es tenso (es decir $r \geq 3$ ) entonces $T$ es isomorfo a una suma de Zykov no trivial.

Demostración. Sea $T$ un torneo regular de orden $2 n+1$. De la demostración del teorema 4.4, observemos que la partición obtenida en (4.7), (4.8), (4.9) y (4.10), definen una suma de Zykov como sigue.

Notemos que

$$
N^{+}\left(X_{1}\right) \backslash\left(X_{1} \cup X_{2}\right)=N^{+}\left(X_{2}\right) \backslash\left(X_{1} \cup X_{2}\right)
$$

$\mathrm{y}$

$$
N^{-}\left(X_{1}\right) \backslash\left(X_{1} \cup X_{2}\right)=N^{-}\left(X_{2}\right) \backslash\left(X_{1} \cup X_{2}\right)
$$

Entonces definimos la suma de Zykov, cuya base $B$ es el torneo que se obtiene al aplicar la proyección $\pi: V(T) \rightarrow V(B)$ al conjunto $X_{1} \cup X_{2}$ en los vértices de $T$, es decir, la base $B$ tiene asociado al vértice $\pi\left(X_{1} \cup X_{2}\right)$ al conjunto $X_{1} \cup X_{2}$ (véase la figura 4.8).

Así la familia $\alpha$ está formada por el torneo $T\left\langle X_{1} \cup X_{2}\right\rangle$ y $\left|X_{3}\right|+\left|X_{4}\right|+\ldots+\left|X_{r}\right|$ veces un vértice singular, es decir

$$
\alpha=\left\{T\left\langle X_{1} \cup X_{2}\right\rangle,\left(\left|X_{3}\right|+\left|X_{4}\right|+\ldots+\left|X_{r}\right|\right) \cdot K_{1}\right\}
$$


y la suma de Zykov es

$$
B\left[\left\{T\left\langle X_{1} \cup X_{2}\right\rangle,\left(\left|X_{3}\right|+\left|X_{4}\right|+\ldots+\left|X_{r}\right|\right) \cdot K_{1}\right\}\right] .
$$

Los siguientes corolarios dan respuesta al siguiente problema propuesto por Víctor Neumann-Lara.

Problema 4.1 (Problema de la página 623 en [NL99]). ¿Cuándo un torneo regular circulante es $\vec{\omega}$-agudo o $\overrightarrow{\omega_{3}}$-agudo?

De hecho, contestamos de manera más general al problema, ya que demostramos que no solo los torneos regulares circulantes son $\overrightarrow{\omega_{3}}$-agudos y $\vec{\omega}$-agudos, sino para torneos regulares en general.

Corolario 4.6. Los torneos regulares son $\overrightarrow{\omega_{3}}-$ agudos.

Demostración. Sean $\varphi: V(T) \rightarrow\left\{X_{1}, X_{2}, \ldots, X_{\vec{\omega}_{3}(T)}\right\}$ una coloración óptima y $x_{1} \in X_{1}$ como en la prueba del teorema 4.4. Observemos que por (4.7), (4.8), (4.9) y (4.10) que

$$
N^{+}\left(X_{1}\right) \backslash\left(X_{1} \cup X_{2}\right)=N^{+}\left(X_{2}\right) \backslash\left(X_{1} \cup X_{2}\right)
$$

y

$$
N^{-}\left(X_{1}\right) \backslash\left(X_{1} \cup X_{2}\right)=N^{-}\left(X_{2}\right) \backslash\left(X_{1} \cup X_{2}\right) .
$$

Definimos la $\overrightarrow{\omega_{3}}(T)$-coloración $\psi: V(T) \rightarrow\left\{Y_{1}, Y_{2}, \ldots, Y_{\overrightarrow{\omega_{3}}(T)}\right\}$ a partir de la coloración $\varphi$ como sigue:

$$
\psi(v)= \begin{cases}Y_{1}, & \text { si } v=x_{1} \\ Y_{2}, & \text { si } v \in\left(X_{1} \backslash\left\{x_{1}\right\}\right) \cup X_{2}, \\ Y_{i}, & \text { si } v \in X_{i}, i \in\left\{3,4, \ldots, \overrightarrow{\omega_{3}}(T)\right\}\end{cases}
$$

que claramente también es óptima y libre de $\overrightarrow{C_{3}}$ heterocromáticos. Más aún, es una coloración con una clase cromática singular, por lo que $T$ es $\overrightarrow{\omega_{3}}$-agudo.

Recordemos que dado un torneo $T$, un $\overrightarrow{C_{4}} \subset T$ no puede estar contenido en tres clases cromáticas distintas. Así, dada una $r$-coloración $\varphi: V(T) \rightarrow\left\{X_{1}, X_{2}, \ldots, X_{r}\right\}$, si deseamos comprobar que esta coloración es libre de $\vec{C}_{4}$ bien coloreados, entonces basta con analizar que entre cualquier par de clases cromáticas, no se inducen $\overrightarrow{C_{4}}$ bien coloreados.

Corolario 4.7. Los torneos regulares son $\vec{\omega}$-agudos.

Demostración. Sea $\psi: V(T) \rightarrow\left\{Y_{1}, Y_{2}, \ldots, Y_{\vec{\omega}_{3}(T)}\right\}$ la coloración definida en (4.11). Dados $i, j$ con $1 \leq i<j \leq \vec{\omega}_{3}(T)$, tenemos que $X_{i}^{+} \cup X_{i}^{-} \Rightarrow X_{j}^{+}$y $X_{j}^{-} \Rightarrow X_{i}^{+} \cup X_{i}^{-}$, por lo que todo par de clases cromáticas $X_{i}$ y $X_{j}$ no inducen $\overrightarrow{C_{4}}$ bien coloreados, lo que demuestra que $\psi$ definida en (4.11) es también libre de $\vec{C}_{4}$ bien coloreados. Esto prueba que la coloración $\psi$ es $\vec{\omega}$-aguda. 
Los colorarios 4.6 y 4.7 son una generalización del siguiente corolario dado por B. Llano y V. Neumann-Lara.

Corolario 4.8 (Corolario 1 de [LlNL07]). Sea $p \geq 3$ un entero primo, entonces el torneo circulante $\vec{C}_{p}(J)$ es $\overrightarrow{\omega_{3}}-$ agudo y $\vec{\omega}$-agudo.

El siguiente corolario contesta afirmativamente y de forma mucho más general al problema 6.6.3 planteado por Neumann-Lara en [NL99], es decir, probamos que la igualdad se cumple para cualquier clase de torneo regular.

Problema 4.2 (Problema 6.6.3 en [NL99]). ¿Se cumple que $\overrightarrow{\omega_{3}}(T)=\vec{\omega}(T)$ para todo torneo $T$ regular o circulante?

Corolario 4.9. Si T es un torneo regular entonces $\vec{\omega}_{3}(T)=\vec{\omega}(T)$.

Demostración. Por el teorema 4.4 y los corolarios 4.6 y 4.7, tenemos que la coloración $\psi$ definida en $(4.11)$ es óptima, libre de $\overrightarrow{C_{3}}$ heterocromáticos y libre de $\left\{\overrightarrow{C_{3}}, \overrightarrow{C_{4}}\right\}$ bien coloreados respectivamente. 


\section{Otros resultados sobre la tensión}

En este capítulo damos una serie de resultados acerca de la tensión, los cuales son consecuencias del capítulo anterior. Primero hacemos un estudio de una subdigráfica asociada a un torneo regular, la cual nos proporciona una segunda caracterización para los torneos regulares tensos y un método para determinar una coloración óptima que realice la inconexión acíclica y la inconexión libre de $\vec{C}_{3}$.

Posteriormente hacemos uso de estos resultados, para dar condiciones necesarias para que un torneo semirregular sea tenso, así como describimos y damos algunos resultados acerca de los torneos regulares que son críticos en vértices. Finalmente proponemos un tipo de gráficas de intersección, que nos ayudarán a dar una tercera caracterización de los torneos regulares tensos. Más aún, esta caracterización nos proporciona un método de obtener la descomposición de un torneo regular primo en una suma de Zykov no trivial.

\subsection{Gráficas bipartitas y torneos regulares tensos}

La siguiente definición de una digráfica bipartita asociada a un torneo regular dado, nos facilitará dar una nueva caracterización de los torneos regulares tensos.

Definición 5.1. Sean $D$ una digráfica y $v \in V(D)$. La digráfica bipartita inducida por $v, B_{v}(D)=(V, A)$ se define sobre los conjuntos

$$
\begin{aligned}
& V\left(B_{v}(D)\right)=V(D) \backslash\{v\} \\
& A\left(B_{v}(D)\right)=\left\{u \rightarrow w \text { en } A(D): u \in N^{+}(v), w \in N^{-}(v)\right\} .
\end{aligned}
$$

En la figura 5.1 se muestra el torneo circulante $\overrightarrow{C_{9}}\langle\emptyset\rangle$ y su digráfica bipartita $B_{0}\left(\overrightarrow{C_{9}}\langle\emptyset\rangle\right)$ asociada. Notemos además que por el lema $1.1^{1}$ de Alspach, toda flecha está en un triángulo dirigido, entonces todo vértice $u \in V(T)$ está en un triángulo dirigido. Lo anterior señala que los vértices de $B_{v}(T)$ no son singulares. Más aún, un vértice $u$ es una fuente en $B_{v}(T)$ si y solo si $u \in N^{+}(v)$ y $u$ es un pozo si y solo si $u \in N^{-}(v)$.

Proposición 5.1. Sean $T$ un torneo regular de orden $2 n+1, v \in V(T)$ y $B_{v}(T)$ la digráfica bipartita inducida por $v$. Si $B_{v}(T)$ tiene una fuente o un pozo u de cardinalidad $n$, entonces el par $\{u, v\}$ es discordante en $T$.

Demostración. Si $u$ es una fuente en $B_{v}(T)$ de cardinalidad $n$, entonces

$$
N^{+}(u, T)=N^{-}(v, T),
$$

\footnotetext{
${ }^{1}$ página 7
} 

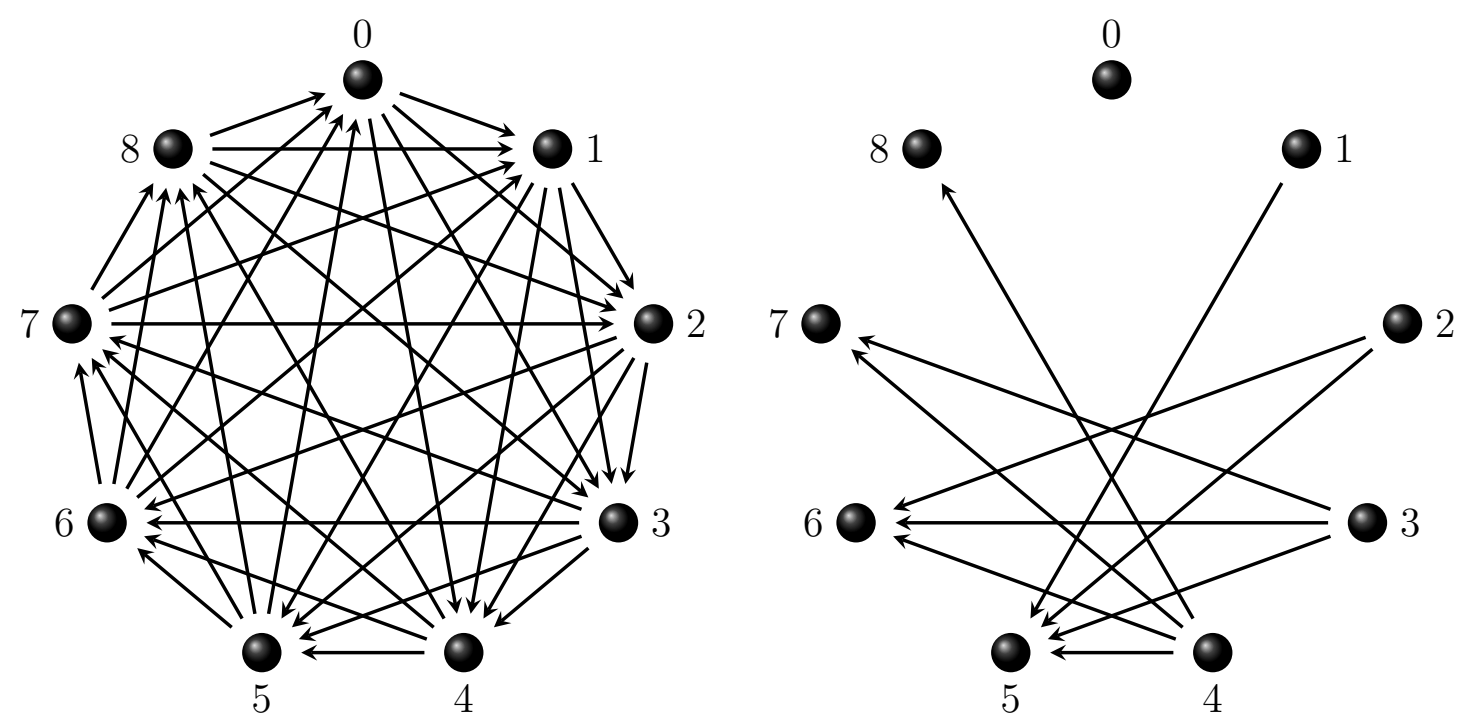

Figura 5.1: El torneo circulante $\overrightarrow{C_{9}}\langle\emptyset\rangle$ y la digráfica $B_{0}\left(\overrightarrow{C_{9}}\langle\emptyset\rangle\right)$.

por lo que

$$
N^{-}(u, T)=N^{+}(v, T),
$$

así el par $\{u, v\}$ es discordante. De manera similar tenemos que si $u$ es un pozo de cardinalidad $n$ en $B_{v}(T)$, entonces

$$
N^{-}(u, T)=N^{+}(v, T) \quad \text { y } \quad N^{+}(u, T)=N^{-}(v, T),
$$

así el par $\{v, u\}$ es discordante en $T$.

El siguiente teorema proporciona otra caracterización de los torneos regulares tensos, la cual está basada en el análisis de la conexidad (débil) de la digráfica bipartita asociada $B_{v}(T)$.

Teorema 5.1. Sea $T$ un torneo regular, entonces $T$ es primo si y solo si $B_{v}(T)$ es débilmente conexa para toda $v \in V(T)$.

Demostración. Demostremos la contrapositiva de esta afirmación, es decir, mostremos que existe $v \in V(T)$ tal que $B_{v}(T)$ no es conexa si y solo si $T$ es isomorfo a una suma de Zykov no trivial.

Para demostrar la suficiencia, supongamos que $T$ es un torneo regular y que existe $v \in V(T)$ tal que $B_{v}(T)$ no es conexa. Sean $B_{1}, B_{2}, \ldots, B_{r}(r \geq 2)$ las componentes conexas de $B_{v}(T)$ y definimos

$$
B_{i}^{+}=B_{i} \cap N^{+}(v) \quad \text { y } \quad B_{i}^{-}=B_{i} \cap N^{-}(v)
$$


para toda $i \in\{1,2, \ldots, r\}$. Como $B_{v}(T)$ no es conexa, entonces las flechas que van de $B_{i}^{+}$a $B_{1}^{-}$no están en $A\left(B_{v}(T)\right)$ para toda $i \in\{2,3, \ldots, r\}$. Tenemos un caso similar para las flechas que van de $B_{1}^{+}$a $B_{i}^{-}$que no están en $A\left(B_{v}(T)\right)$. Así

$$
B_{1}^{-} \Rightarrow \bigcup_{i=2}^{r} B_{i}^{+} \quad \text { y } \quad B_{1}^{+} \Leftarrow \bigcup_{i=2}^{r} B_{i}^{-}
$$

en $T$ (véase la figura 5.2).

Definimos la coloración $\varphi: V(T) \rightarrow\left\{X_{1}, X_{2}, X_{3}\right\}$ como

$$
\varphi(u)= \begin{cases}X_{1}, & \text { si } u=v, \\ X_{2}, & \text { si } u \in B_{1}, \\ X_{3}, & \text { si } u \in B_{i}, i \in\{2,3, \ldots, r\}\end{cases}
$$

que claramente es una 3-coloración libre de $\overrightarrow{C_{3}}$ heterocromáticos (no necesariamente óptima). Por el teorema $4.2^{2}$, tenemos que $T$ no es primo y por lo tanto es isomorfo a una suma de Zykov no trivial.

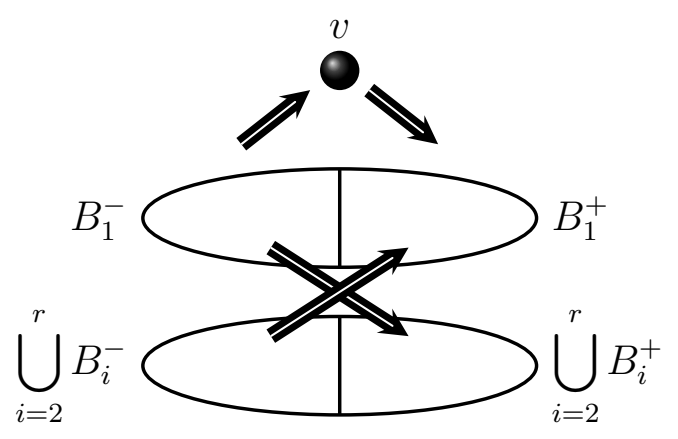

Figura 5.2: La digráfica bipartita $B_{v}(T)$.

Para demostrar la necesidad, supongamos que $T \cong T^{\prime}[\alpha]$ es isomorfo a una suma de Zykov no trivial. Sea $\alpha=\left\{\alpha_{i}\right\}_{i \in V\left(T^{\prime}\right)}$ la familia de torneos de la suma y sin pérdida de generalidad, supongamos que $\alpha_{v}$ es el torneo no trivial en $\alpha$ asociado al vértice $v \in V\left(T^{\prime}\right)$ tal que $\pi\left(\alpha_{v}\right)=v$, donde $\pi: V(T) \rightarrow V\left(T^{\prime}\right)$ es el epimorfismo reflexivo descrito en la definición $1.7^{3}$.

Sea $v_{1} \in V\left(\alpha_{v}\right)$. Por el lema $1.3^{4}$, tenemos que el torneo $\alpha_{v}$ es regular y es claro que $B_{v_{1}}\left(\alpha_{v}\right)$ es no vacía, pues al menos existe un triángulo dirigido que contiene al vértice $v_{1}$. Por otra parte, notemos también que para toda flecha contenida en la base $T^{\prime}$, esta se encuentra contenida en un triángulo dirigido.

Por lo anterior, tenemos que para todo vértice $u \in V\left(T^{\prime}\right) \cap N^{+}\left(v, T^{\prime}\right)$, existe un vértice $w \in V\left(T^{\prime}\right) \cap N^{-}\left(v, T^{\prime}\right)$, tal que $\overrightarrow{C_{3}}: v \rightarrow u \rightarrow w \rightarrow v$ está en la base $T^{\prime}$.

\footnotetext{
${ }^{2}$ página 64

${ }^{3}$ página 11

${ }^{4}$ página 14
} 
Entonces $\alpha_{u} \Rightarrow \alpha_{w}$, por lo que la digráfica $B_{v_{1}}\left(T\left\langle\alpha_{u} \cup \alpha_{w} \cup\left\{v_{1}\right\}\right\rangle\right)$ es una componente conexa (débil) en $B_{v_{1}}(T)$ y es claro que

$$
V\left(\alpha_{u}\right) \subseteq N^{+}\left(v_{1}\right) \quad \text { y } \quad V\left(\alpha_{w}\right) \subseteq N^{-}\left(v_{1}\right) .
$$

Más aún, tenemos que $\alpha_{v} \Rightarrow \alpha_{u}$ y $\alpha_{w} \Rightarrow \alpha_{v}$, por lo que estas flechas no forman triángulo dirigido alguno con $v_{1}$ y en particular

$$
V\left(\alpha_{v}\right) \cap N^{-}\left(v_{1}\right) \Rightarrow V\left(\alpha_{u}\right) \quad \text { y } \quad V\left(\alpha_{v}\right) \cap N^{+}\left(v_{1}\right) \Leftarrow V\left(\alpha_{w}\right) .
$$

Por lo tanto no hay trayectoria alguna de $V\left(\alpha_{u}\right) \cap N^{+}\left(v_{1}\right)$ a $V\left(\alpha_{v}\right) \cap N^{-}\left(v_{1}\right)$ y de manera similar, se tiene que tampoco hay trayectorias alguna de $V\left(\alpha_{v}\right) \cap N^{+}\left(v_{1}\right)$ a $V\left(\alpha_{w}\right) \cap N^{-}\left(v_{1}\right)$.

Concluimos que $B_{v_{1}}(T)$ no es conexa (débil) para $v_{1} \in V(T)$.

Con la ayuda del teorema 5.1, demostramos el siguiente teorema que relaciona la inconexión libre de $\overrightarrow{C_{3}}$ y las $\omega$ componentes conexas (débiles) de la digráfica bipartita $B_{v}(T)$.

Teorema 5.2. Sea $T$ un torneo regular, entonces

$$
\vec{\omega}_{3}(T)=\operatorname{máx}\left\{\omega\left(B_{v}(T)\right): v \in V(T)\right\}+1
$$

Demostración. El caso cuando $\overrightarrow{\omega_{3}}(T)=2$ es directo, ya que $T$ es primo si y solo si es tenso y por el teorema 5.1, se tiene que $B_{v}(T)$ es conexa (débil) para toda $v \in V(T)$, entonces $\omega\left(B_{v}(T)\right)=1$ y se cumple lo requerido.

Supongamos que $\overrightarrow{\omega_{3}}(T) \geq 3$ y por el teorema $5.1, T$ es isomorfo a una suma de Zykov no trivial $T^{\prime}[\alpha]$, donde $\alpha=\left\{\alpha_{i}\right\}_{i \in V\left(T^{\prime}\right)}$ es la familia de torneos regulares. Por el corolario $4.6^{5}$, existe una coloración $\overrightarrow{\omega_{3}}$-aguda $\varphi: V(T) \rightarrow\left\{X_{1}, X_{2}, \ldots, X_{\overrightarrow{\omega_{3}}(T)}\right\}$ libre de $\vec{C}_{3}$ heterocromáticos y sin pérdida de generalidad supongamos que $X_{1}=\{v\}$ es la clase cromática singular.

Mostremos la igualdad mediante las dos desigualdades.

- Similarmente a la demostración del teorema $4.4^{6}$, definimos los conjuntos

$$
X_{i}^{+}=X_{i} \cap N^{+}(v) \quad \text { y } \quad X_{i}^{-}=X_{i} \cap N^{-}(v)
$$

para toda $i \in\left\{1,2, \ldots, \overrightarrow{\omega_{3}}(T)\right\}$. Así

$$
\bigcup_{\substack{i=1 \\ i \neq j}}^{\overrightarrow{\omega_{3}}(T)} X_{i}^{+} \Leftarrow X_{j}^{-} \quad \mathrm{y} \quad \bigcup_{\substack{i=1 \\ i \neq j}}^{\overrightarrow{\omega_{3}}(T)} X_{i}^{-} \Rightarrow X_{j}^{+},
$$

\footnotetext{
${ }^{5}$ página 74

${ }^{6}$ página 68
} 
por lo que cada clase cromática induce al menos una componente (no necesariamente conexa) en $B_{v}(T)$ y por lo tanto tenemos al menos $\omega\left(B_{v}(T)\right.$ ) componentes conexas (débiles). Como $v$ es una clase cromática singular, tenemos que

$$
\overrightarrow{\omega_{3}}(T) \leq \operatorname{máx}\left\{\omega\left(B_{v}(T)\right): v \in V(T)\right\}+1 .
$$

- Por otra parte supongamos que $B_{1}, B_{2}, \ldots, B_{r}$ son las componentes conexas (débiles) de $B_{v}(T)$, con $r \geq 3$. Por la construcción del teorema 4.4, las particiones

$$
N^{+}(v)=B_{1}^{+}\left|B_{2}^{+}\right| \cdots \mid B_{r}^{+} \quad \text { y } \quad N^{-}(v)=B_{1}^{-}\left|B_{2}^{-}\right| \cdots \mid B_{r}^{-}
$$

son externamente libres de $\overrightarrow{C_{3}}$, por lo que la partición

$$
V(T)=\{v\}\left|B_{1}\right| B_{2}|\cdots| B_{r}
$$

es también externamente libre de $\overrightarrow{C_{3}}$. La coloración $\varphi: V(T) \rightarrow\left\{X_{1} X_{2}, \ldots, X_{r}\right\}$ definida por

$$
\psi(u)= \begin{cases}X_{1}, & \text { si } u=v, \\ X_{i}, & \text { si } u \in B_{i}, i \in\{2,3, \ldots, r\},\end{cases}
$$

es libre de $\overrightarrow{C_{3}}$ heterocromáticos (no necesariamente óptima) y así

$$
r=\omega\left(B_{v}(T)\right)+1 \leq \overrightarrow{\omega_{3}}(T)
$$

para toda $v \in V(T)$, por lo que

$$
\operatorname{máx}\left\{\omega\left(B_{v}(T)\right): v \in V(T)\right\}+1 \leq \overrightarrow{\omega_{3}}(T)
$$

Por las desigualdades 5.2 y 5.3 concluimos que

$$
\overrightarrow{\omega_{3}}(T)=\operatorname{máx}\left\{\omega\left(B_{v}(T)\right): v \in V(T)\right\}+1 .
$$

El teorema anterior, proporciona un método para determinar la inconexión libre de $\overrightarrow{C_{3}}$ de un torneo $T$ de orden $2 n+1$, esto es, puede ser calculada con la digráfica bipartita $B_{v}(T)$, al tomar el máximo número de componentes conexas (débiles) para toda $v \in V(T)$.

Notemos que de esta forma, se reduce a analizar solo $2 n+1$ digráficas, en contraste con lo que supondría analizar todas las particiones de $V(T)$. Esto proporciona una ventaja al problema, puesto que analizar todas las particiones posibles de $V(T)$ es un problema exponencial.

Como ejemplo de lo anterior, sea el torneo $\vec{C}_{3}\left[\overrightarrow{C_{3}}\right]$ de la figura 5.3. Notemos que para $\{0\} \in V\left(\overrightarrow{C_{3}}\left[\overrightarrow{C_{3}}\right]\right)$, la digráfica $B_{0}\left(\overrightarrow{C_{3}}\left[\overrightarrow{C_{3}}\right]\right)$ tiene tres componentes conexas (débiles), que proporcionan la partición

$$
V\left(\overrightarrow{C_{3}}\left[\overrightarrow{C_{3}}\right]\right)=\{0\}|\{3,6\}|\{1,2,4,5,7,8\} .
$$




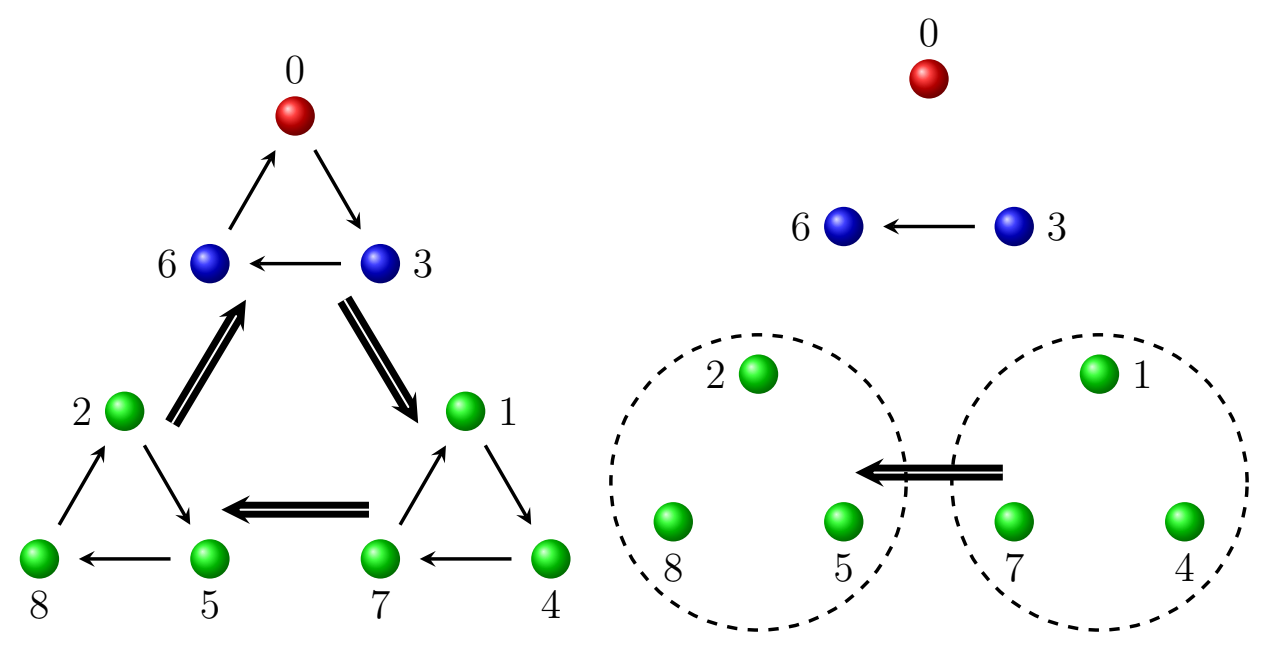

Figura 5.3: El torneo $\overrightarrow{C_{3}}\left[\overrightarrow{C_{3}}\right]$ y la digráfica $B_{0}\left(\overrightarrow{C_{3}}\left[\overrightarrow{C_{3}}\right]\right)$.

Notemos que esta partición induce una 3-coloración óptima libre de $\overrightarrow{C_{3}}$ heterocromáticos, al colorear el vértice singular y los vértices de cada componente conexa (débil) de un color distinto. No es difícil de verificar que se cumple el teorema 5.2.

Por otra parte, el teorema 5.1 proporciona una nueva caracterización para los torneos regulares tensos, que se suma a la caracterización dada en el teorema 4.2 de la página 64, la cual se resume en el siguiente teorema.

Teorema 5.3 (Caracterización de los torneos regulares primos). Las siguientes proposiciones son equivalentes para un torneo regular $T$.

i) $T$ es primo.

ii) $T$ es tenso.

iii) $B_{v}(T)$ es conexa (débil) para toda $v \in V(T)$.

Demostración. Se sigue por los teoremas 4.2 y 5.1 .

\subsection{Inconexión acíclica de torneos semirregulares}

Recordemos que un torneo $T=(V, A)$ es semirregular-también llamado torneo casi regular- si

$$
\left|d^{+}(v)-d^{-}(v)\right|=1
$$

para toda $v \in V(T)$.

Más aún, si $T$ es un torneo semirregular, entonces tiene orden par $2 n$ y $d^{+}(v)=n-1$ o $d^{+}(v)=n$ para todo vértice $v \in V(T)$. Por lo anterior se puede concluir que si $d^{+}(v)=n-1$, entonces $d^{-}(v)=n$ y similarmente si $d^{+}(v)=n$ entonces $d^{-}(v)=n-1$. 
Recordemos que el vector de ex-grados (resp. vector de in-grados) de un torneo $T$ de orden $n$, es una sucesión de enteros no negativos $\left(s_{1}, s_{2}, \ldots, s_{n}\right)$, donde $s_{i}$ es el número de ex-vecinos (resp. in-vecinos) del vértice $v_{i}$, donde $i \in\{1,2, \ldots, n\}$, es decir $s_{i}=d^{+}\left(v_{i}\right)$ (resp. $\left.s_{i}=d^{-}\left(v_{i}\right)\right)$.

El primer resultado fue tomado de [R04], el cual es un lema muy conocido sobre torneos. Dicho lema caracteriza la existencia de un torneo con vector de ex-grados dado (o equivalentemente de in-grados).

Lema 5.1. Una sucesión de $n$ enteros $\left(s_{1}, s_{2}, \ldots, s_{n}\right)$, donde $s_{1} \leq s_{2} \leq \ldots \leq s_{n}$, es el vector de ex-grados (resp. in-grados) de algún torneo de orden $n$ si y solo si

$$
\sum_{i=1}^{k} s_{i} \geq\left(\begin{array}{l}
k \\
2
\end{array}\right), \text { para } k \in\{1,2, \ldots, n-1\} \quad y \quad \sum_{i=1}^{n} s_{i}=\left(\begin{array}{l}
n \\
2
\end{array}\right)
$$

Con la ayuda del lema anterior, probaremos el siguiente lema, que es una propiedad interesante de los torneos semirregulares, el cual nos dice que hay igual número de vértices con ex-grado $n$ que con ex-grado $n-1$.

Lema 5.2. Sea $T$ un torneo semirregular de orden $2 n$ y definamos la partición de $V(T)=P_{1} \mid P_{2}$, donde

$$
\begin{aligned}
& P_{1}=\left\{v \in V(T): d^{+}(v)=n-1\right\} \quad y \\
& P_{2}=\left\{v \in V(T): d^{+}(v)=n\right\}
\end{aligned}
$$

entonces $\left|P_{1}\right|=\left|P_{2}\right|$.

Demostración. Sin pérdida de generalidad, supongamos que $\left|P_{1}\right| \leq\left|P_{2}\right|$, por lo que si $\left|P_{1}\right|=m \leq n$, entonces $\left|P_{2}\right|=2 n-m$. Como el torneo es semirregular, entonces su vector de ex-grados es de la forma

$$
(\underbrace{n-1, n-1, \ldots, n-1}_{m \text { veces }}, \underbrace{n, n, \ldots, n}_{(2 n-m) \text { veces }})
$$

entonces por el lema 5.1 tenemos que

$$
\sum_{i=1}^{2 n} s_{i}=m(n-1)+(2 n-m) n=\left(\begin{array}{c}
2 n \\
2
\end{array}\right)
$$

lo que se reduce a resolver la ecuación

$$
m(n-1)+(2 n-m) n=\left(\begin{array}{c}
2 n \\
2
\end{array}\right)
$$

cuya única solución es $m=n$. Por lo tanto $\left|P_{1}\right|=\left|P_{2}\right|$. 
El siguiente lema da una conexión interesante entre un torneo regular y uno semirregular, ya que dado un torneo regular $T$, a partir de él podemos construir un torneo semirregular al eliminar cualquier vértice.

Similarmente, dado un torneo semirregular $S R$, entonces podemos «completarlo»a un torneo regular, al agregar un vértice con flechas apropiadas.

Lema 5.3. Sean $T$ un torneo regular de orden $2 n+1 y v \in V(T)$, entonces el torneo residual inducido

$$
T\langle V(T) \backslash\{v\}\rangle
$$

es un torneo semirregular de orden $2 n$.

Similarmente, si $S R$ es un torneo semirregular y dado un vértice $v \notin V(S R)$, entonces el torneo aumentado $T$ definido por

$$
\begin{aligned}
& V(T)=V(S R) \cup\{v\}, \\
& A(T)=A(S R) \cup A_{v}
\end{aligned}
$$

donde

$$
A_{v}= \begin{cases}v \rightarrow u, & \text { si } d^{+}(u)=n-1, \\ v \leftarrow u, & \text { si } d^{+}(u)=n,\end{cases}
$$

para toda $u \in V(S R)$, es un torneo regular.

Demostración. Sean $T$ un torneo regular de orden $2 n+1$ y $v$ un vértice de $T$. Es claro que a cada vértice de $N^{+}(v)$ y de $N^{-}(v)$, se le ha disminuido en una unidad a su número de in-vecinos y ex-vecinos respectivamente en el torneo residual $T\langle V(T) \backslash\{v\}\rangle$, el cual resulta ser semirregular.

Para el segundo caso, si $S R$ es un torneo semirregular y definimos la partición de $V(S R)=P_{1} \mid P_{2}$ donde

$$
\begin{aligned}
& P_{1}=\left\{u \in V(S R): d^{+}(u)=n-1\right\}, \\
& P_{2}=\left\{u \in V(S R): d^{+}(u)=n\right\}
\end{aligned}
$$

y hacemos $v \Rightarrow P_{2}$ y $P_{1} \Rightarrow v$, entonces el torneo aumentado $T$ es regular.

Notemos que si el torneo regular $T$ es circulante, entonces su grupo de automorfismos actúa transitivamente en su conjunto de vértices, por lo que el torneo semirregular residual $S R=T\langle V(T) \backslash\{v\}\rangle$ es único para todo vértice $v \in V(T)$, en el sentido que todos los torneos resultantes son isomorfos. Esto no necesariamente sucede en general, ya que si el torneo no es transitivo en vértices, entonces el torneo residual depende del vértice $v$ elegido. En contraste, a todo torneo semirregular $S R$, le asociamos un único torneo regular aumentado $T$.

El siguiente lema es una consecuencia del lema $4.2^{7}$. Notemos que en escencia es el mismo lema, salvo algunos detalles que involucran el orden del torneo, así mismo su demostración es similar. Este lema nos ayudará a probar más adelante, algunas propiedades para torneos semirregulares.

\footnotetext{
${ }^{7}$ página 54
} 
Lema 5.4. Sean $T$ un torneo semirregular de orden $2 n$ y $\varphi: V(T) \rightarrow\{X, Y, Z\}$ una 3-coloración libre de $\overrightarrow{C_{3}}$ heterocromáticos tal que $|X| \leq|Y| \leq|Z|$, entonces

$$
N^{+}\left(N^{+}\left(N^{+}(X) \cap Y\right) \cap Z\right) \cap X=\emptyset
$$

$y$

$$
N^{+}\left(N^{+}\left(N^{+}(Y) \cap X\right) \cap Z\right) \cap Y=\emptyset .
$$

Demostración. Sin pérdida de generalidad, supongamos que $1 \leq|X| \leq\left\lfloor\frac{2 n}{3}\right\rfloor$ y

- $2 \leq|Y| \leq\left\lfloor\frac{2 n}{3}\right\rfloor$, si $|T| \equiv 0,1$ (mód 3) $\quad$ o

- $2 \leq|Y| \leq\left\lceil\frac{2 n}{3}\right\rceil$, si $|T| \equiv 2$ (mód 3).

Supongamos por contradicción que

$$
N^{+}\left(N^{+}\left(N^{+}(X) \cap Y\right) \cap Z\right) \cap X \neq \emptyset .
$$

Con los mismos argumentos de la demostración del lema 4.2, podemos construir la trayectoria

$$
P: x^{\prime} \rightarrow y \rightarrow z \rightarrow x,
$$

donde $\left\{x, x^{\prime}\right\} \subset X, y \in Y$ y $z \in Z$. Dado el vértice $x^{\prime}$ de la trayectoria $P$, notemos que $N^{+}\left(x^{\prime}\right)=n$ y $N^{+}\left(x^{\prime}\right)=n-1$ o bien, $N^{+}\left(x^{\prime}\right)=n-1$ y $N^{+}\left(x^{\prime}\right)=n$.

Corolario 5.1. Sea $T$ un torneo semirregular con $\vec{\omega}_{3}(T)=r$. Entonces $T$ no es tenso (es decir $r \geq 3$ ) si y solo si $T$ es isomorfo a una suma de Zykov no trivial.

Demostración. La necesidad se sigue del lema 5.4 y la suficiencia del corolario 1.2.

Observación 5.1. El corolario 5.1 señala que si $T$ es un torneo regular no tenso, entonces esta propiedad se hereda al torneo semirregular $S R=T \backslash\{v\}$ para toda $v \in X_{i}$, ya que si la coloración

$$
\varphi: V(T) \rightarrow\left\{X_{1}, X_{2}, \ldots, X_{\vec{\omega}_{3}(T)}\right\}
$$

es libre de $\overrightarrow{C_{3}}$ heterocromáticos, tal que $2 \leq\left|X_{1}\right| \leq\left|X_{2}\right| \leq \ldots \leq\left|X_{\vec{\omega}_{3}(T)}\right|$, entonces para el torneo semirregular $S R=T \backslash\{v\}$, se tiene que la coloración heredada

$$
\psi: V(S R) \rightarrow\left\{X_{1}, X_{2}, \ldots, X_{\vec{\omega}_{3}(T)}\right\}
$$

es también libre de $\overrightarrow{C_{3}}$ heterocromáticos.

Similarmente si $\varphi$ es $\overrightarrow{\omega_{3}}$-aguda y redefiniendo las clases cromáticas $X_{1}^{\prime}=X_{2} \cap N^{+}(v)$ y $X_{2}^{\prime}=X_{2} \cap N^{-}(v)$, tenemos que $\psi: V(S R) \rightarrow\left\{X_{1}^{\prime}, X_{2}^{\prime}, X_{3}, \ldots, X_{\omega_{3}(T)}\right\}$ es también libre de $\overrightarrow{C_{3}}$ heterocromáticos. 
Lo anterior también tiene su equivalente en términos de sumas de Zykov, ya que si el torneo regular $T$ es una suma de Zykov no trivial, entonces el torneo residual $T \backslash\{v\}$ también lo es, para todo vértice $v$ de las componentes no triviales de la suma.

Por ejemplo, sea la composición $\vec{C}_{3}\left[\vec{C}_{3}\right]$ y sin pérdida de generalidad sea $\{0\} \in V(T)$. Es claro que el torneo residual $S R=\overrightarrow{C_{3}}\left[\overrightarrow{C_{3}}\right] \backslash\{0\}$ sigue siendo una suma de Zykov no trivial, cuya base $B$ se obtiene al aplicar el epimorfismo reflexivo $\pi: V\left(\overrightarrow{C_{3}}\left[\overrightarrow{C_{3}}\right]\right) \rightarrow V(B)$ a la flecha $3 \rightarrow 6$. Notemos en la figura 5.4 que redefiniendo la coloración de la forma

$$
X_{1}^{\prime}=X_{2} \cap N^{+}(0)=\{3\} \quad \mathrm{y} \quad X_{2}^{\prime}=X_{2} \cap N^{-}(0)=\{6\},
$$

como en la observación 5.1, entonces obtenemos una coloración óptima libre de $\overrightarrow{C_{3}}$ heterocromáticos.

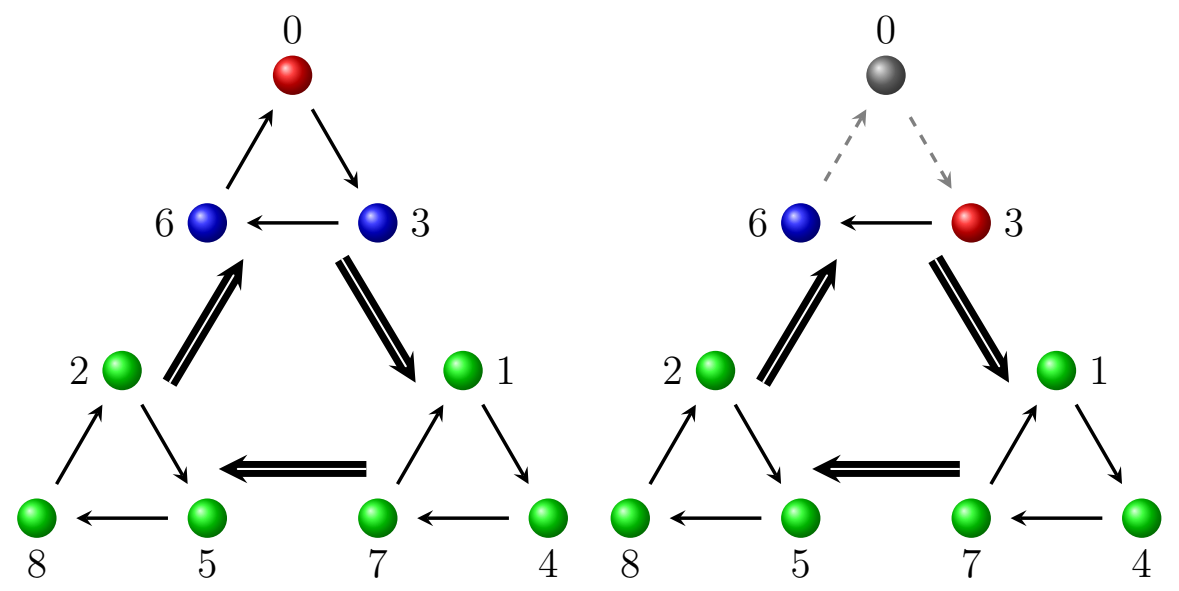

Figura 5.4: El torneo $\overrightarrow{C_{3}}\left[\overrightarrow{C_{3}}\right]$ y su torneo residual $\overrightarrow{C_{3}}\left[\overrightarrow{C_{3}}\right] \backslash\{0\}$.

En contraste con lo anterior, la propiedad de primalidad para un torneo regular $T$, no necesariamente se hereda al torneo semirregular $S R=T \backslash\{v\}$, para algún vértice $v$.

Por ejemplo, sea el torneo cíclico $\overrightarrow{C_{9}}\langle\emptyset\rangle$ de la figura 5.5, el cual sabemos que es tenso por el inciso (i) de la observación $3.2^{8}$. Este torneo no hereda la propiedad de primalidad, ya que el torneo semirregular $\overrightarrow{C_{9}}\langle\emptyset\rangle \backslash\{0\}$ no es primo, pues podemos aplicar el epimorfismo reflexivo $\pi: V\left(\overrightarrow{C_{3}}\left[\overrightarrow{C_{3}}\right]\right) \rightarrow V(B)$ a la flecha $4 \rightarrow 5$ y así obtenemos la base $B$ de una suma de Zykov no trivial. Para el torneo semirregular $S R=\overrightarrow{C_{9}}\langle\emptyset\rangle \backslash\{0\}$, la 3-coloración $\psi: V(S R) \rightarrow\left\{X_{1}, X_{2}, X_{3}\right\}$ definida por

$$
\varphi(v)= \begin{cases}X_{1}, & \text { si } v=4, \\ X_{2}, & \text { si } v=5 \\ X_{3}, & \text { en otro caso, }\end{cases}
$$

es libre de $\overrightarrow{C_{3}}$ heterocromáticos, ya que la flecha $5 \rightarrow 4$ no pertenece a triángulo dirigido alguno.

\footnotetext{
${ }^{8}$ página 41
} 

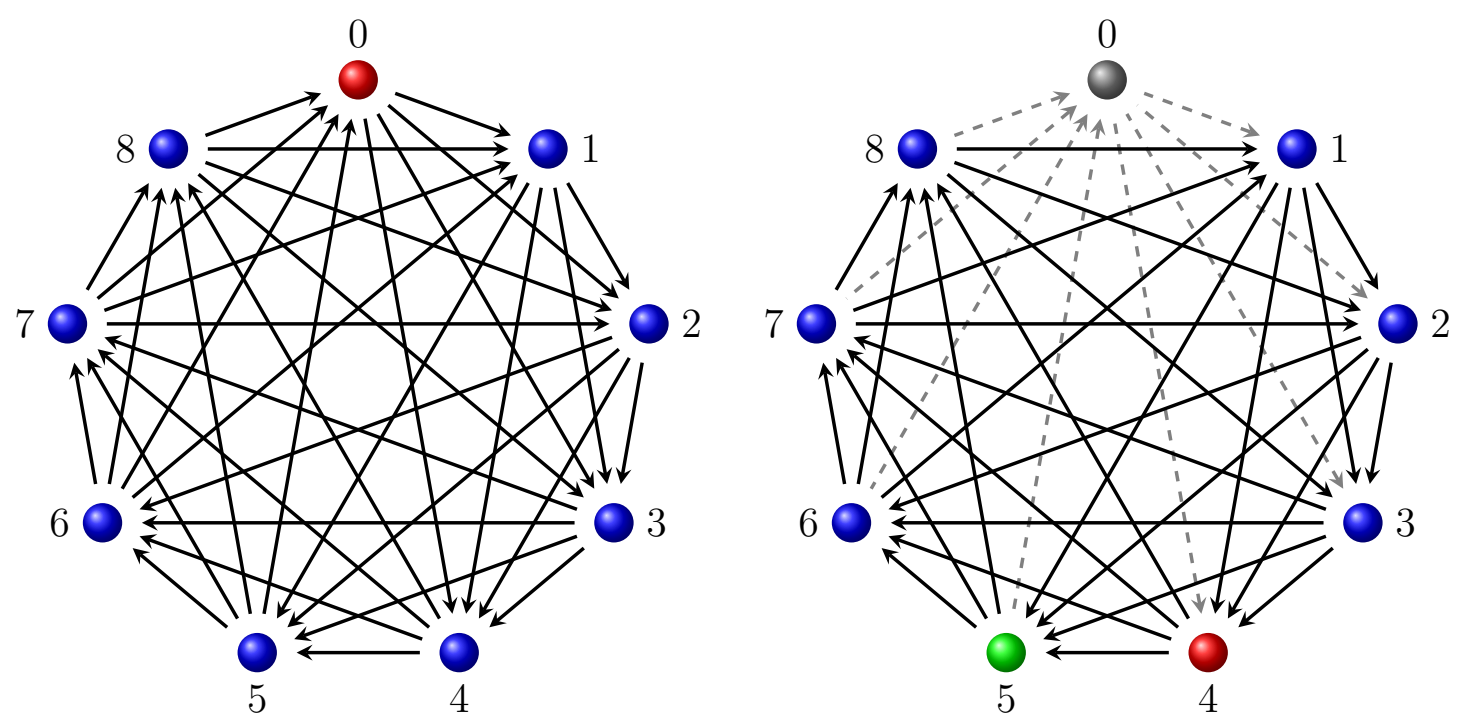

Figura 5.5: El torneo $\overrightarrow{C_{9}}\langle\emptyset\rangle$ y su torneo residual $\overrightarrow{C_{9}}\langle\emptyset\rangle \backslash\{0\}$.

\subsection{Torneos regulares críticos}

El corolario 5.1 y los ejemplos anteriores, mostrados en las figuras 5.4 y 5.5, dan pauta para preguntarnos acerca de la clase de torneos regulares, cuya propiedad de primalidad no es heredada al torneo semirregular residual. En otras palabras, nos interesaría saber en qué clase de torneos regulares, la primalidad -y por lo tanto la tensión-, no se hereda al torneo residual.

Este tipo de problema ha sido estudiado antes para otro parámetro que mide la estructura cíclica de las digráficas: el número dicromático.

Recordemos que en [NL82], Víctor Neumann-Lara define el número dicromático $d c(D)$ de una digráfica $D$, como el mínimo número de colores necesarios para colorear los vértices de la digráfica $D$, de tal manera que cada clase cromática sea acíclica.

Una digráfica $D$ tal que $d c(D)=r$, la llamaremos $r$-dicromática.

En [NU83] Víctor Neumann-Lara y Jorge Urrutia, dan una clase de torneos regulares para los cuales el número dicromático no se hereda al torneo semirregular residual. Estamos en la búsqueda de aquellos torneos cuya propiedad de primalidad no se hereda con respeto a la inconexión acíclica y la inconexión libre de $\overrightarrow{C_{3}}$.

Para el estudio de esta propiedad, tenemos la siguiente.

Definición 5.2. Sean $D$ una digráfica y $\alpha(D)$ un parámetro asociado a la digráfica $D$. Decimos que la digráfica $D$ es crítica en vértices respecto al parámetro $\alpha$, si para toda $v \in V(T)$ se tiene que

$$
\alpha(D) \neq \alpha(D \backslash\{v\})
$$


En particular, si para un torneo $T$ se tiene que

$$
r=d c(T)>d c(T \backslash\{v\})
$$

para todo vértice $v \in V(T)$, entonces decimos que el torneo $T$ es $r$-dicromático crítico en vértices.

Similarmente, para la inconexión acíclica y la inconexión libre de $\vec{C}_{3}$, si

$$
\overrightarrow{\omega_{3}}(T)<\overrightarrow{\omega_{3}}(T \backslash\{v\}) \quad \text { о } \quad \vec{\omega}(T)<\vec{\omega}(T \backslash\{v\}) .
$$

para toda $v \in V(T)$, entonces decimos que el torneo $T$ es $\overrightarrow{\omega_{3}}$-crítico o $\vec{\omega}$-crítico en vértices respectivamente.

En 1984, Neumann-Lara y Urrutia abordaron el problema de determinar clases de torneos regulares $r$-dicromáticos críticos en vértices y mostraron el siguiente teorema. El lector interesado puede encontrar la demostración en [NU83].

Teorema 5.4 (Teorema 1 en [NU83]). Si $T$ es un torneo regular de orden $2 n+1$, entonces $d c(T)=2$ si y solo si $T \cong \vec{C}_{2 n+1}\langle\emptyset\rangle$.

El teorema anterior señala que el único torneo regular que induce dos particiones internamente acíclicas, es el torneo cíclico $\vec{C}_{2 n+1}\langle\emptyset\rangle$. Como el único torneo acíclico es el torneo transitivo, entonces dicha partición induce copias isomorfas de los torneos transitivos de orden $n$ y $n+1$. Por ejemplo $T\left\langle N^{+}(0) \cup\{0\}\right\rangle \cong T T_{n+1}$ y $T\left\langle N^{-}(0)\right\rangle \cong T T_{n}$.

El teorema anterior permite demostrar el siguiente teorema sobre una clase de torneos regulares 3-dicromáticos críticos en vértices.

Teorema 5.5 (Teorema 2 en [NU83]). El torneo circulante $\vec{C}_{2 n+1}\langle n\rangle$ es 3-dicromático crítico en vértices, para toda $n \geq 3$.

Demostración. Sin pérdida de generalidad sea $\{0\} \in V\left(\vec{C}_{2 n+1}\langle n\rangle\right)$. Notemos que

$$
\vec{C}_{2 n+1}\langle\emptyset\rangle\langle\{1,2, \ldots, n\}\rangle \cong \vec{C}_{2 n+1}\langle n\rangle\langle\{1,2, \ldots, n\}\rangle \cong T T_{n}
$$

pero

$$
\vec{C}_{2 n+1}\langle\emptyset\rangle\langle\{0,1,2, \ldots, n\}\rangle \supsetneqq \vec{C}_{2 n+1}\langle n\rangle\langle\{0,1,2, \ldots, n\}\rangle,
$$

ya que el primero es acíclico y el segundo no lo es, por lo que $\vec{C}_{2 n+1}\langle\emptyset\rangle \supsetneqq \vec{C}_{2 n+1}\langle n\rangle$. Por los argumentos anteriores y por el teorema 5.4 , tenemos que $d c\left(\vec{C}_{2 n+1}\langle n\rangle\right) \geq 3$. La coloración $\psi: V\left(\vec{C}_{2 n+1}\langle n\rangle\right) \rightarrow\left\{X_{1}, X_{2}, X_{3}\right\}$ definida por

$$
\varphi(v)= \begin{cases}X_{1}, & \text { si } u=\{0\}, \\ X_{2}, & \text { si } u \in\{1,2, \ldots, n\}, \\ X_{3}, & \text { si } u \in\{n+1, n+2, \ldots, 2 n\},\end{cases}
$$

induce subtorneos acíclicos monocromáticos, por lo que $d c\left(\vec{C}_{2 n+1}\langle n\rangle\right)=3$. Así mismo, notemos que $d c\left(\vec{C}_{2 n+1}\langle n\rangle \backslash\{0\}\right)=2$ y dado que $\vec{C}_{2 n+1}\langle n\rangle \backslash\{0\} \cong \vec{C}_{2 n+1}\langle n\rangle \backslash\{v\}$ para toda $v \in V\left(\vec{C}_{2 n+1}\langle n\rangle\right)$, se tiene que es 3 -dicromático crítico en vértices. 

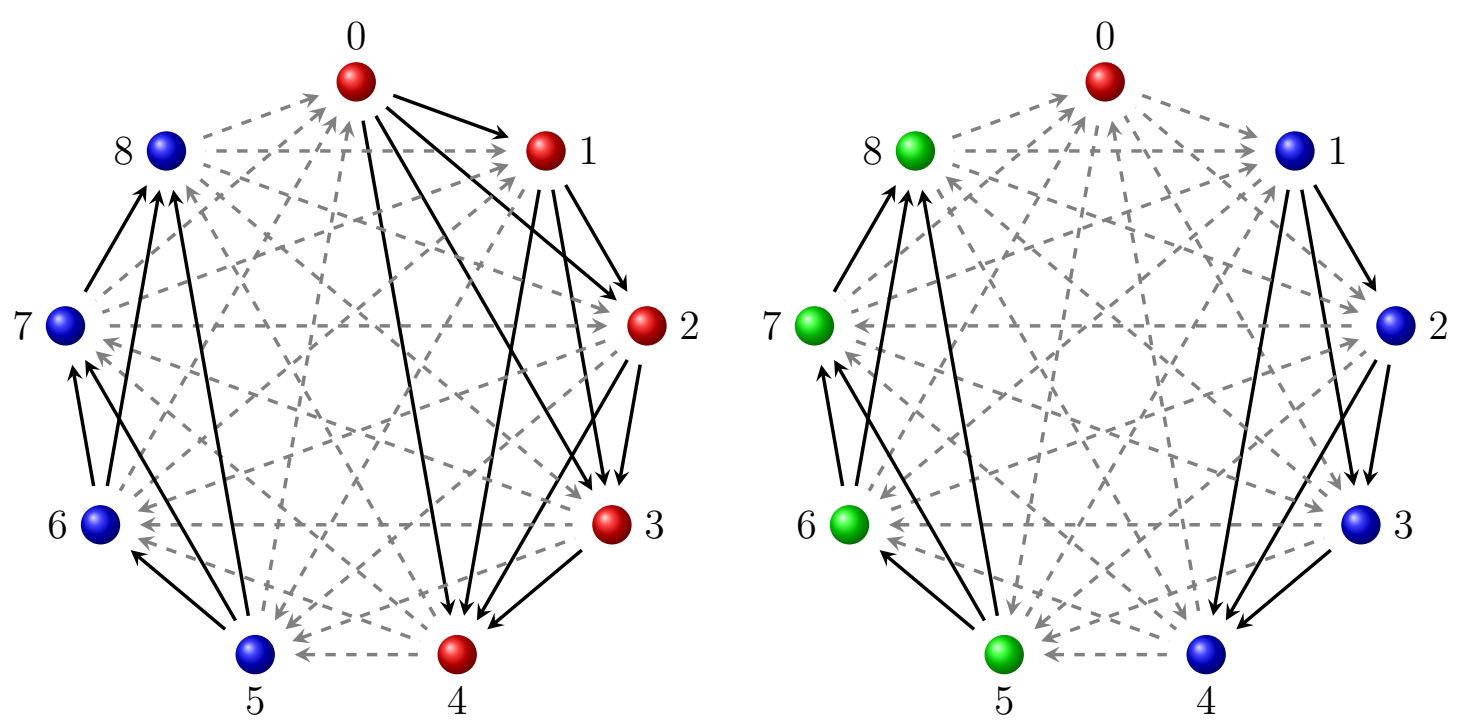

Figura 5.6: Una coloración que realiza el $d c$ de los torneos $\overrightarrow{C_{9}}\langle\emptyset\rangle$ y $\overrightarrow{C_{9}}\langle 4\rangle$.

En la figura 5.6 podemos observar los torneos $\vec{C}_{9}\langle\emptyset\rangle$ y $\vec{C}_{9}\langle 4\rangle$ con coloraciones que realizan el número dicromático $d c\left(\vec{C}_{9}\langle\emptyset\rangle\right)=2$ y $d c\left(\vec{C}_{9}\langle 4\rangle\right)=3$.

En [NU83] se proponen otras clases de torneos que son 3-dicromáticos críticos en vértices, los cuales no trataremos en esta tesis. Siguiendo la idea de estos teoremas sobre torneos críticos, damos el siguiente teorema que muestra una clase de torneos regulares que son $\overrightarrow{\omega_{3}}-$ críticos en vértices.

Teorema 5.6. El torneo regular cíclico $\vec{C}_{2 n+1}\langle\emptyset\rangle$ es $\vec{\omega}_{3}$-crítico en vértices.

Demostración. Sin pérdida de generalidad sea $\{0\} \in V\left(\vec{C}_{2 n+1}\langle\emptyset\rangle\right)$. Notemos en la figura 5.7 que por la construcción del torneo, la flecha $n \rightarrow n+1$ pertenece solo al triángulo dirigido $\overrightarrow{C_{3}}: 0 \rightarrow n \rightarrow(n+1) \rightarrow 0$, por lo que dicha flecha no está en triángulo alguno en $\vec{C}_{2 n+1}\langle\emptyset\rangle \backslash\{0\}$. La coloración $\psi: V\left(\vec{C}_{2 n+1}\langle\emptyset\rangle \backslash\{0\}\right) \rightarrow\left\{X_{1}, X_{2}, X_{3}\right\}$ definida por

$$
\varphi(u)= \begin{cases}X_{1}, & \text { si } u=\{n\}, \\ X_{2}, & \text { si } u=\{n+1\}, \\ X_{3}, & \text { en otro caso. }\end{cases}
$$

es libre de $\overrightarrow{C_{3}}$ heterocromáticos. Dado que $\vec{C}_{2 n+1}\langle\emptyset\rangle \backslash\{0\} \cong \vec{C}_{2 n+1}\langle\emptyset\rangle \backslash\{v\}$ para toda $v \in V\left(\vec{C}_{2 n+1}\langle\emptyset\rangle\right)$, se tiene que es $\overrightarrow{\omega_{3}}$-crítico.

En la figura 5.7 podemos observar a $\vec{C}_{2 n+1}\langle\emptyset\rangle \backslash\{0\}$ y la coloración (5.5) como ejemplo de un torneo que es $\overrightarrow{\omega_{3}}$-crítico en vértices. Así mismo, la estructura del torneo 


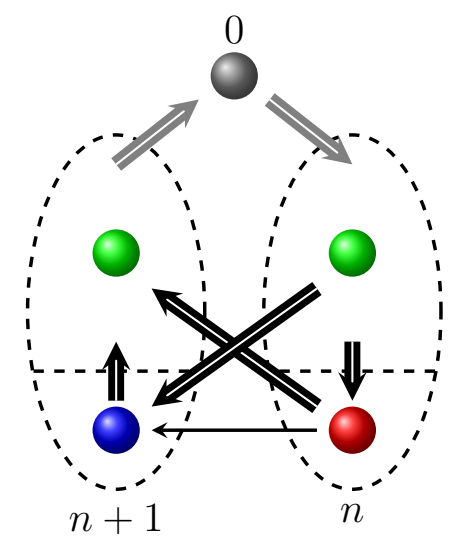

Figura 5.7: El torneo $\vec{C}_{2 n+1}\langle\emptyset\rangle \backslash\{0\}$ es $\vec{\omega}_{3}$-crítico en vértices.

$\vec{C}_{2 n+1}\langle\emptyset\rangle \backslash\{0\}$ permite dar el siguiente resultado que involucra a la digráfica bipartita $B_{v}(T)$ de la definición $5.1^{9}$.

Teorema 5.7. Sea $T$ un torneo regular de orden $2 n+1$. Entonces $T \cong \vec{C}_{2 n+1}\langle\emptyset\rangle$ si $y$ solo si la digráfica bipartita $B_{v}(T)$ tiene una fuente y un pozo de cardinalidad $n$, para toda $v \in V(T)$.

Demostración. Para demostrar la necesidad, supongamos que $T \cong \vec{C}_{2 n+1}\langle\emptyset\rangle$ y $\sin$ pérdida de generalidad, sea $v=\{0\}$. Como $n \in N^{+}(0)$ y por la definición del torneo cíclico, tenemos que

$$
\begin{aligned}
N^{+}(n) & =\{1,2, \ldots, n\}+n \\
& =\{n+1, n+2, \ldots, 2 n\} \\
& =N^{-}(0) .
\end{aligned}
$$

Similarmente para $n+1 \in N^{-}(0)$ tenemos que

$$
\begin{aligned}
N^{-}(n+1) & =\mathbb{Z}_{2 n} \backslash N^{+}(n) \\
& =\{1,2, \ldots, n\} \\
& =N^{+}(0) .
\end{aligned}
$$

Así, $n$ es una fuente y $n+1$ es un pozo en $B_{0}\left(\vec{C}_{2 n+1}\langle\emptyset\rangle\right)$, ambos de cardinalidad $n$. Más aún, para toda $i \in \mathbb{Z}_{2 n+1}$ tenemos que $i+n$ es una fuente e $i+n+1$ es un pozo de cardinalidad $n$ en $B_{i}\left(\vec{C}_{2 n+1}\langle\emptyset\rangle\right)$.

Para mostrar la suficiencia, sea $v_{0} \in V(T)$ tal que existen $u_{1}, u_{2} \in V(T)$ tal que $u_{1}$ y $u_{2}$ son la fuente y el pozo respectivamente, ambos de cardinalidad $n$ en $B_{v_{0}}(T)$. Claramente $u_{1} \in N^{+}\left(v_{0}\right)$ y $u_{2} \in N^{-}\left(v_{0}\right)$, ademas observemos en la figura 5.8 que

\footnotetext{
${ }^{9}$ página 77
} 


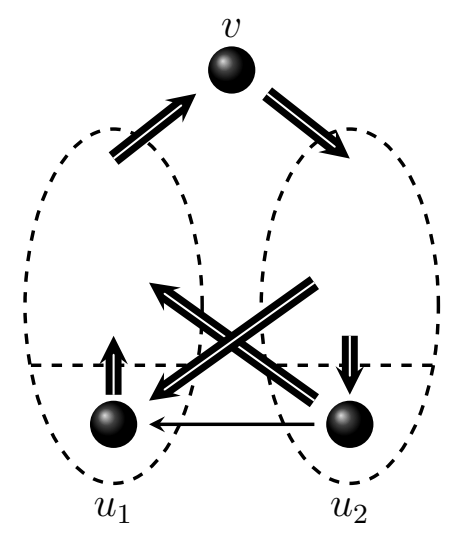

Figura 5.8: La digráfica $B_{v}(T)$ con una fuente $u_{1}$ y un pozo $u_{2}$.

$N^{+}\left(u_{1}\right)=N^{-}(v)$ y $N^{-}\left(u_{2}\right)=N^{+}(v)$, entonces los pares $\left\{u_{1}, v\right\}$ y $\left\{v, u_{2}\right\}$ son discordantes.

Sin pérdida de generalidad, supongamos que

$$
P_{1}: v_{1} \rightarrow v_{2} \rightarrow \ldots \rightarrow v_{n}
$$

es una trayectoria generadora para $N^{+}\left(v_{0}\right)$ tal que

$$
\left|N^{+}\left(v_{1}, B_{v_{0}}(T)\right)\right| \leq\left|N^{+}\left(v_{2}, B_{v_{0}}(T)\right)\right| \leq \ldots \leq\left|N^{+}\left(v_{n}, B_{v_{0}}(T)\right)\right| .
$$

Similarmente, supongamos que

$$
P_{2}: v_{n+1} \rightarrow v_{n+2} \rightarrow \ldots \rightarrow v_{2 n}
$$

es una trayectoria generadora para $N^{-}\left(v_{0}\right)$ tal que

$$
\left|N^{+}\left(v_{2 n}, B_{v_{0}}(T)\right)\right| \leq\left|N^{+}\left(v_{2 n-1}, B_{v_{0}}(T)\right)\right| \leq \ldots \leq\left|N^{+}\left(v_{n+1}, B_{v_{0}}(T)\right)\right|
$$

Por la definición de las trayectorias anteriores, notemos que $v_{n} \mathrm{y} v_{n+1}$ son la fuente y el pozo de cardinalidad $n$ en $B_{v_{0}}(T)$, por lo que

$$
N^{+}\left(v_{0}, T\right) \backslash\left\{v_{n}\right\} \Rightarrow v_{n} \quad \mathrm{y} \quad N^{-}\left(v_{0}, T\right) \backslash\left\{v_{n+1}\right\} \Leftarrow v_{n+1} .
$$

Por otra parte, observemos que $v_{n+1} \in N^{+}\left(v_{1}, B_{v_{1}}(T)\right)$ y $v_{0} \in N^{-}\left(v_{1}, B_{v_{1}}(T)\right)$. Como $u_{1} \Rightarrow N^{-}\left(v_{0}, T\right)$ entonces

$$
\left|N^{+}\left(v_{2 n}, B_{v_{1}}(T)\right)\right| \leq\left|N^{+}\left(v_{2 n-1}, B_{v_{1}}(T)\right)\right| \leq \ldots \leq\left|N^{+}\left(v_{n+2}, B_{v_{1}}(T)\right)\right| .
$$

Más aún, como $N^{-}\left(u_{0}, T\right) \Rightarrow v_{0}$ entonces $\left|N^{+}\left(v_{0}, B_{v_{1}}(T)\right)\right| \leq\left|N^{+}\left(v_{2 n}, B_{v_{1}}(T)\right)\right|$ y así

$$
\left|N^{+}\left(v_{0}, B_{v_{1}}(T)\right)\right| \leq\left|N^{+}\left(v_{2 n}, B_{v_{1}}(T)\right)\right| \leq \ldots \leq\left|N^{+}\left(v_{n+2}, B_{v_{1}}(T)\right)\right|,
$$


por lo que $v_{n+1}$ y $v_{n+2}$ son la fuente y el pozo respectivamente, ambos de cardinalidad $n$ en $B_{v_{1}}(T)$. Así $N^{+}\left(v_{1}, T\right) \backslash\left\{v_{n+1}\right\} \Rightarrow v_{n+1}$ y $N^{-}\left(v_{1}, T\right) \backslash\left\{v_{n+2}\right\} \Leftarrow v_{n+2}$.

Repitiendo el argumento anterior para cada vértice $v_{i}$, con $i \in \mathbb{Z}_{2 n+1}$, tenemos que $N^{+}\left(v_{i}\right)=\left\{v_{i+1}, v_{i+2}, \ldots, v_{i+n}\right\}$, por lo tanto $N^{-}\left(v_{i}\right)=\left\{v_{i-1}, v_{i-2}, \ldots, v_{i-n}\right\}$ (tomando las sumas módulo $2 n+1)$.

La función $\phi: V\left(\vec{C}_{2 n+1}\langle\emptyset\rangle\right) \rightarrow V(T)$ definida por

$$
\phi(i)=v_{i}
$$

es un isomorfismo. Por lo tanto $T \cong \vec{C}_{2 n+1}\langle\emptyset\rangle$.

Finalmente tenemos el siguiente corolario para la inconexión acíclica.

Corolario 5.2. Sea el torneo circulante $\vec{C}_{2 n+1}\langle\emptyset\rangle$, entonces $\vec{\omega}\left(\vec{C}_{2 n+1}\langle\emptyset\rangle \backslash\{0\}\right)=3$.

Demostración. Demostremos que la coloración $\varphi: V\left(\vec{C}_{2 n+1}\langle\emptyset\rangle \backslash\{0\}\right) \rightarrow\left\{X_{1}, X_{2}, X_{3}\right\}$

$$
\varphi(v)= \begin{cases}X_{1}, & \text { si } v=\{n\} \\ X_{2}, & \text { si } v=\{n+1\} \\ X_{3}, & \text { en otro caso. }\end{cases}
$$

definida en (5.5) de la demostración del teorema 5.6, es libre de $\vec{C}_{4}$ bien coloreados. Notemos en la figura 5.7 que para que un $\overrightarrow{C_{4}}$ dirigido esté bien coloreado, debería estar contenido alternadamente en al menos dos clases cromáticas. Sin embargo esto no es posible, ya que los únicos $\overrightarrow{C_{4}}$ que pasan por dos clases cromáticas son aquellos que contienen a la flecha $n \rightarrow n+1$.

$$
\begin{gathered}
\text { Como } N^{+}(n+1)=\{n+2, n+3, \ldots, 2 n\}, N^{-}(n)=\{1,2, \ldots, n-1\} \mathrm{y} \\
\psi(\{n+2, n+3, \ldots, 2 n\})=\psi(\{1,2, \ldots, n-1\}),
\end{gathered}
$$

entonces los cuadrados dirigidos no están bien coloreados. Por lo tanto, la coloración (5.5) es libre de $\left\{\overrightarrow{C_{3}}, \overrightarrow{C_{4}}\right\}$ heterocromáticos.

Esto demuestra que $\vec{\omega}\left(\vec{C}_{2 n+1}\langle\emptyset\rangle \backslash\{0\}\right)=\vec{\omega}_{3}\left(\vec{C}_{2 n+1}\langle\emptyset\rangle \backslash\{0\}\right)=3$.

El siguiente corolario resume los dos resultados anteriores.

Corolario 5.3. El torneo circulante $\vec{C}_{2 n+1}\langle\emptyset\rangle$ es $\vec{\omega}_{3}$-crítico y $\vec{\omega}$-crítico en vértices.

Demostración. Por la observación 3.2 y el corolario $4.9^{10}$ se sigue que

$$
\vec{\omega}_{3}\left(\vec{C}_{2 n+1}\langle\emptyset\rangle\right)=\vec{\omega}\left(\vec{C}_{2 n+1}\langle\emptyset\rangle\right)=2 .
$$

\footnotetext{
${ }^{10}$ páginas 41 y 75 respectivamente
} 
Por otra parte, por el teorema 5.6 y el corolario 5.2 tenemos que para todo vértice $v \in V\left(\vec{C}_{2 n+1}\langle\emptyset\rangle\right)$

$$
\vec{\omega}_{3}\left(\vec{C}_{2 n+1}\langle\emptyset\rangle \backslash\{v\}\right)=\vec{\omega}\left(\vec{C}_{2 n+1}\langle\emptyset\rangle \backslash\{v\}\right)=3
$$

lo que demuestra que $\vec{C}_{2 n+1}\langle\emptyset\rangle$ es $\vec{\omega}_{3}$-crítico y $\vec{\omega}$-crítico en vértices.

El corolario anterior proporciona una clase de torneos regulares primos y que son $\overrightarrow{\omega_{3}}$-críticos y $\vec{\omega}$-críticos en vértices. Sin embargo no sabemos si son únicos, ya que de serlo, tendríamos que dado un torneo semirregular $S R$, entonces $\overrightarrow{\omega_{3}}(S R)=\vec{\omega}(S R)$.

\subsection{Factorización de torneos y la gráfica $G_{3}(D)$}

En esta sección definimos un nuevo tipo de gráficas de intersección a partir de una digráfica dada, en particular, nos enfocaremos en el estudio la gráfica asociada a un torneo regular.

La gráfica $G_{3}(T)$ permite dar una caracterización para torneos regulares primos, pero más aún, nos proporciona una manera sencilla de decidir si un torneo regular es o no una suma de Zykov no trivial, a partir del análisis de la conexidad de la misma. Dicha factorización proporcionada por esta caracterización, nos permite dar una descomposición de un torneo regular en componentes de una suma de Zykov no trivial.

Definición 5.3. Sea $D$ una digráfica. Definimos la gráfica de intersección de triángulos $G_{3}(D)$, sobre los conjuntos

$$
\begin{aligned}
& V\left(G_{3}(D)\right)=\left\{v_{\{i, j, k\}}:\{i, j, k\} \subset V(D), D\langle\{i, j, k\}\rangle \cong \vec{C}_{3}\right\}, \\
& E\left(G_{3}(D)\right)=\left\{v_{\{i, j, k\}} u_{\left\{i^{\prime}, j^{\prime}, k^{\prime}\right\}}:\left|\{i, j, k\} \cap\left\{i^{\prime}, j^{\prime}, k^{\prime}\right\}\right|=2\right\} .
\end{aligned}
$$

Es decir, los vértices de la gráfica $G_{3}(D)$ serán ternas de vértices de $D$ que inducen un $\overrightarrow{C_{3}}$ en $D$. A los vértices de $G_{3}(D)$ les llamaremos 3-vértices y declararemos adyacentes a los 3-vértices $e_{\{i, j, k\}}$ y $e_{\left\{i^{\prime}, j^{\prime}, k^{\prime}\right\}}$ en $G_{3}(D)$, si los triángulos $\vec{C}_{3}: i \rightarrow j \rightarrow k \rightarrow i$ $y \overrightarrow{C_{3}}: i^{\prime} \rightarrow j^{\prime} \rightarrow k^{\prime} \rightarrow i^{\prime}$ comparten una flecha en $D$.

Notemos que la gráfica $G_{3}(D)$ depende de la estructura cíclica de la digráfica $D$, en específico, depende de la estructura de triángulos, por lo que una condición necesaria para que $G_{3}(D)$ sea no vacía, es que $D$ contenga al menos un triángulo dirigido.

Por ejemplo, sea el torneo regular $\vec{C}_{5}\langle\emptyset\rangle$ y notemos que

$$
\overrightarrow{C_{3}}: 0 \rightarrow 2 \rightarrow 3 \rightarrow 0 \quad \text { y } \quad \overrightarrow{C_{3}}: 0 \rightarrow 2 \rightarrow 4 \rightarrow 0
$$

son dos triángulos dirigidos, por lo que estas dos ternas de vértices serán los 3-vértices $v_{\{0,2,3\}}$ y $v_{\{0,2,4\}}$ respectivamente en la gráfica $G_{3}\left(\vec{C}_{5}\langle\emptyset\rangle\right)$. Como ambos triángulos tienen 

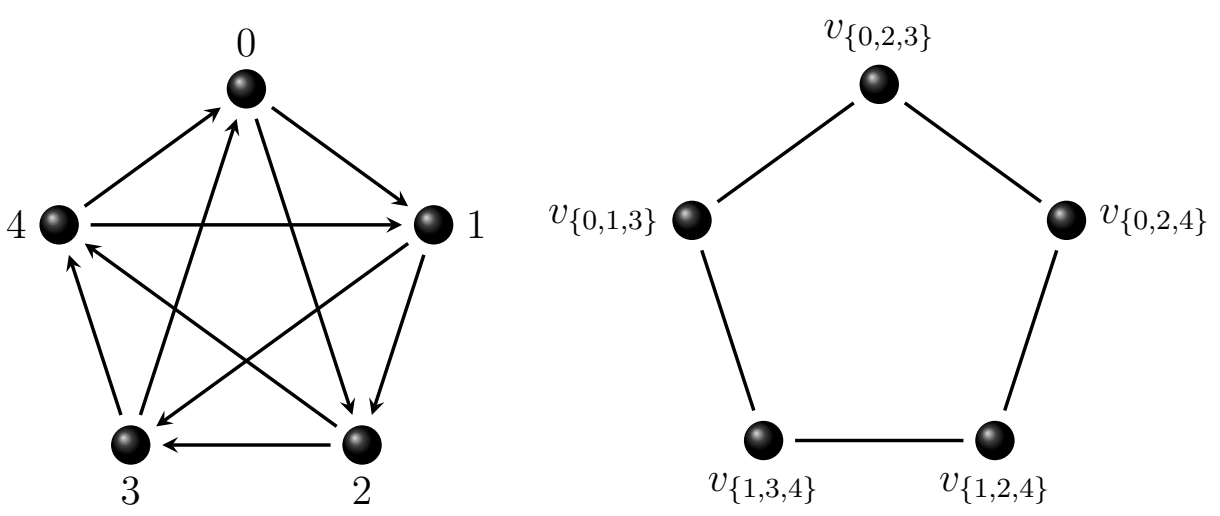

Figura 5.9: El torneo $\vec{C}_{5}\langle\emptyset\rangle$ y la gráfica $G_{3}\left(\vec{C}_{5}\langle\emptyset\rangle\right)$.

en común a la flecha $0 \rightarrow 2$, entonces ambos 3 -vértices son vecinos en $G_{3}\left(\vec{C}_{5}\langle\emptyset\rangle\right)$. Similarmente tenemos otros tres triángulos distintos y procedemos de igual manera para finalmente obtener el ciclo no dirigido $C_{5}$ de la figura 5.9.

Recordemos que todo torneo $T$ fuertemente conexo es pancíclico, es decir, tiene ciclos de todas las longitudes menores o iguales a su orden $|T|$, así dado un torneo $T$, entonces $G_{3}(T)$ es no vacía si y solo si $T$ es fuertemente conexo. Por otra parte, si $T$ es un torneo regular de orden $(2 n+1)$, entonces

$$
\left|G_{3}(T)\right|=\left(\begin{array}{c}
2 n+1 \\
3
\end{array}\right)-(2 n+1)\left(\begin{array}{l}
n \\
2
\end{array}\right),
$$

que es el número de triángulos distintos contenidos en $T$.

Si el torneo $T$ es el triángulo $\vec{C}_{3}$, entonces la gráfica $G_{3}\left(\vec{C}_{3}\right)=K_{1}$, es decir, el vértice singular. Si el torneo $T$ es el torneo $\vec{C}_{5}\langle\emptyset\rangle$, entonces $G_{3}\left(\vec{C}_{5}\langle\emptyset\rangle\right)=C_{5}$, es decir el ciclo no dirigido de longitud cinco. Como $\vec{C}_{5}\langle\emptyset\rangle$ es circulante, entonces notemos que el ciclo $C_{5}$ resulta también ser regular. Esta observación permite dar la siguiente propiedad.

Proposición 5.2. Si $T$ es un torneo circulante de orden $2 n+1$, entonces la gráfica $G_{3}(T)$ es regular.

Demostración. Como $T$ es un torneo circulante, entonces toda flecha $u \rightarrow v$ está en un número fijo de triángulos, más aún, dicho $\overrightarrow{C_{3}}$ comparte sus flechas con un número fijo de triángulos. Así se llega a que $G_{3}(T)$ es regular.

La siguiente proposición permite dar una condición necesaria para identificar si una digráfica es una suma de Zykov no trivial.

Proposición 5.3. Sea $D \cong B[\alpha]$ una digráfica isomorfa a una suma de Zykov no trivial. Supongamos que al menos dos de las digráficas de la familia $\alpha$ son no triviales con al menos un $\overrightarrow{C_{3}}$ cada una de ellas, entonces $G_{3}(D)$ no es conexa. 
Demostración. Supongamos sin pérdida de generalidad, que $\alpha_{1}$ y $\alpha_{2}$ son las dos digráficas no triviales en la suma de Zykov $B[\alpha]$ y que están asociadas a los vértices $v_{1}$ y $v_{2}$ respectivamente en la base $B$. Como las digráficas $\alpha_{1}$ y $\alpha_{2}$ tienen al menos un $\overrightarrow{C_{3}}$ cada uno, entonces $G_{3}\left(\alpha_{1}\right)$ y $G_{3}\left(\alpha_{2}\right)$ son no vacías.

Si entre los vértices $v_{1}$ y $v_{2}$ no hay flecha alguna, entonces es claro que se obtiene lo requerido. En otro caso, supongamos sin pérdida de generalidad que $v_{1} \rightarrow v_{2}$, por lo que $V\left(\alpha_{1}\right) \Rightarrow V\left(\alpha_{2}\right)$. Así, no hay triángulos que compartan flecha alguna entre ellos, por lo que $\alpha_{1}$ y $\alpha_{2}$ inducen componentes no conexas distintas en $G_{3}(T)$.

De igual manera, dado $u_{j} \in V(B)$ entonces $\alpha_{1} \Rightarrow \alpha_{j}$ o $\alpha_{1} \Leftarrow \alpha_{j}$. Esto señala que no hay triángulos en distintas componentes conexas que compartan alguna flecha entre ellos, por lo que no hay trayectoria alguna entre $G_{3}\left(\alpha_{1}\right)$ y $G_{3}\left(\alpha_{j}\right)$. Así $G_{3}(T)$ no es conexa.

La condición de que hayan por lo menos dos componentes no triviales y fuertes en la familia $\alpha$, es necesaria si la base $B$ no es fuerte, ya que de lo contrario puede resultar conexa, por ejemplo, si $T=B[\alpha]$ es una suma de Zykov, donde $B=T T_{n} \mathrm{y}$

$$
\alpha=\left\{\vec{C}_{5}\langle\emptyset\rangle, T T_{i_{1}}, T T_{i_{2}}, \ldots, T T_{i_{n-1}}\right\},
$$

donde $i_{j} \in \mathbb{N}$, es claro que $G_{3}(T) \cong C_{5}$ y por lo tanto es conexa.

Notemos que el recíproco de la proposición 5.3 anterior, no necesariamente es cierto para torneos no regulares, es decir, si $T$ es un torneo no regular y la gráfica $G_{3}(T)$ es conexa, entonces no necesariamente $T$ es una suma de Zykov no trivial. Como ejemplo de lo anterior tenemos el torneo $T_{9} \in \mathfrak{V}_{n}$ de la figura 2.5 de la página 29, el cual no es regular y cuya gráfica $G_{3}\left(T_{9}\right)$ resulta ser conexa.

De la definición de la gráfica $G_{3}(T)$ para un torneo $T$, tenemos que existe una trayectoria del 3-vértice $e_{\left\{v_{1}, u_{1}, w_{1}\right\}}$ al 3-vértice $e_{\left\{v_{n}, u_{n}, w_{n}\right\}}$ en la gráfica $G_{3}(T)$ si y solo si entre los triángulos del torneo $T$,

$$
{\overrightarrow{C_{3}}}^{(1)}: v_{1} \rightarrow u_{1} \rightarrow w_{1} \rightarrow v_{1} \quad \text { y } \quad{\overrightarrow{C_{3}}}^{(n)}: v_{n} \rightarrow u_{n} \rightarrow w_{n} \rightarrow v_{n},
$$

existe una sucesión de triángulos ${\overrightarrow{C_{3}}}^{(1)},{\overrightarrow{C_{3}}}^{(2)}, \ldots, \vec{C}_{3}(n)$ tales que

$$
\left|V\left({\overrightarrow{C_{3}}}^{(i)}\right) \cap V\left({\overrightarrow{C_{3}}}^{(i+1)}\right)\right|=2
$$

para toda $i \in\{1,2, \ldots, n-1\}$. Es decir, existe una sucesión de triángulos distinto no ajenos en flechas.

Sean $G$ y $H$ dos gráficas. El producto cartesiano $G \square H$ se define sobre los conjuntos

$$
\begin{aligned}
V(G \square H) & =V(G) \times V(H), \\
E(G \square H) & =\{(u, v)(x, y): u=x \text { y } v y \in E(H), \text { о } u x \in E(G) \text { y } v=y\} .
\end{aligned}
$$

Es decir, el conjunto de vértices de $G \square H$, es el producto cartesiano de los conjuntos de vértices de sus factores y declaramos adyacentes a los vértices - que son pares ordenados- 
$(u, v)$ y $(x, y)$ si $u=x$ y $v y$ es una arista de $H$ o si $v=y$ y $u x$ es una arista de $G$. De manera inductiva, podemos definir para las gráficas $G_{1}, G_{2}, \ldots, G_{n}$, el producto cartesiano

$$
G_{1} \square G_{2} \square \ldots \square G_{i}=\left(G_{1} \square G_{2} \square \ldots \square G_{i-1}\right) \square G_{i},
$$

para $i \in\{3,4, \ldots, n\}$. El lector interesado en los conceptos, propiedades y otros ejemplos sobre este y otros tipos de productos de gráficas, puede consultar [IKH11].

En la figura 5.10 se muestra el producto cartesiano del ciclo $C_{6}$ y la arista $K_{2}$, así como el correspondiente entre el triángulo $C_{3}$ con él mismo.
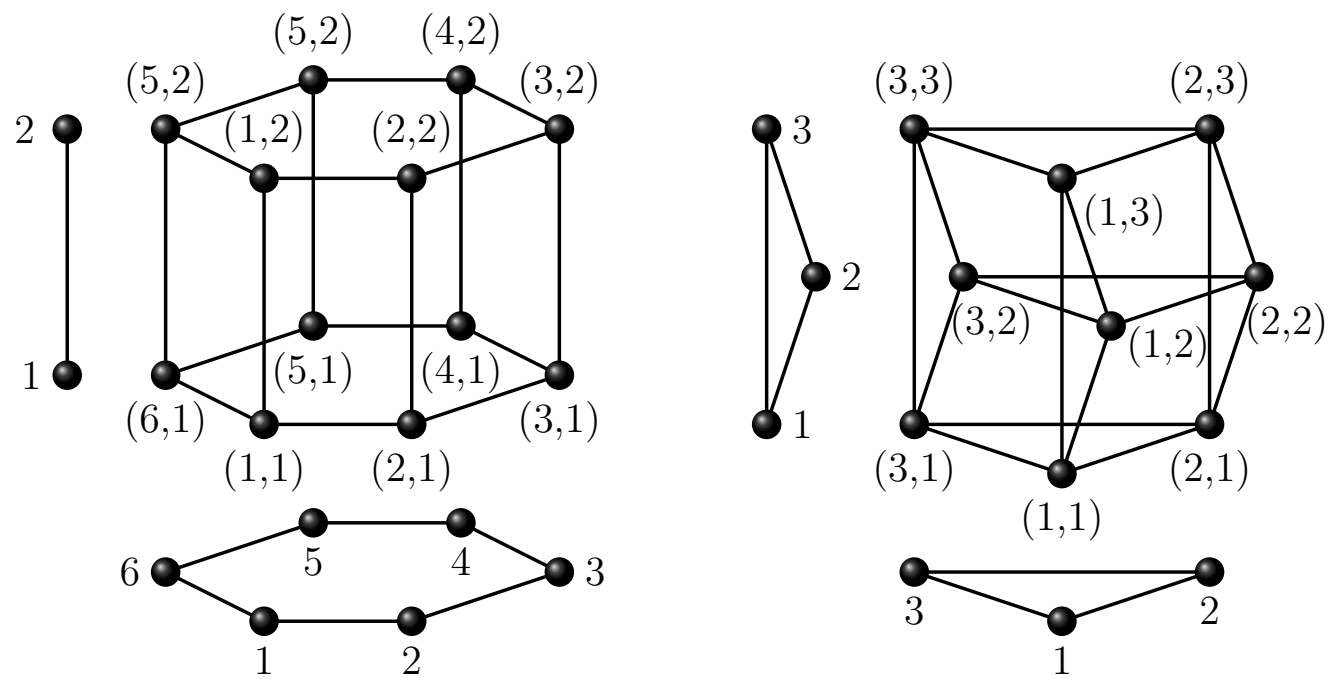

Figura 5.10: Los productos cartesianos $C_{6} \square K_{2}$ y $C_{3} \square C_{3}$.

Sea la composición de $\overrightarrow{C_{3}}\left[\overrightarrow{C_{3}}\right]$ de la figura $5.4^{11}$. En la figura 5.11 se muestra la gráfica $G_{3}\left(\vec{C}_{3}\left[\vec{C}_{3}\right]\right)$, la cual tiene cuatro componentes conexas. Notemos que estas componentes conexas son el producto cartesiano $C_{3} \square C_{3} \square C_{3}$ y tres vértices singulares.

El hecho que $G_{3}\left(\vec{C}_{3}\left[\vec{C}_{3}\right]\right)$ no sea conexa no es una casualidad, ya que si se trata de torneos regulares, tenemos el siguiente teorema que es consecuencia de la definición de la digráfica bipartira $B_{v}(T)$ para un torneo $T$ y un vértice $v \in V(T)$.

Teorema 5.8. Sea $T$ un torneo regular primo, entonces $G_{3}(T)$ es conexa.

Demostración. Sean $v \in V(T)$,

$$
{\overrightarrow{C_{3}}}^{(1)}: v \rightarrow u_{1} \rightarrow w_{1} \rightarrow v \quad \text { y } \quad \vec{C}_{3}^{(n)}: v \rightarrow u_{n} \rightarrow w_{n} \rightarrow v
$$

triángulos distintos en $T$. Por el teorema $5.1^{12}$, tenemos que $B_{v}(T)$ es conexa, esto es, la gráfica bipartita subyacente de $B_{v}(T)$ es conexa. Así tenemos que existe una $u_{1} \rightsquigarrow w_{1}$

\footnotetext{
${ }^{11}$ página 86

12 página 78
} 


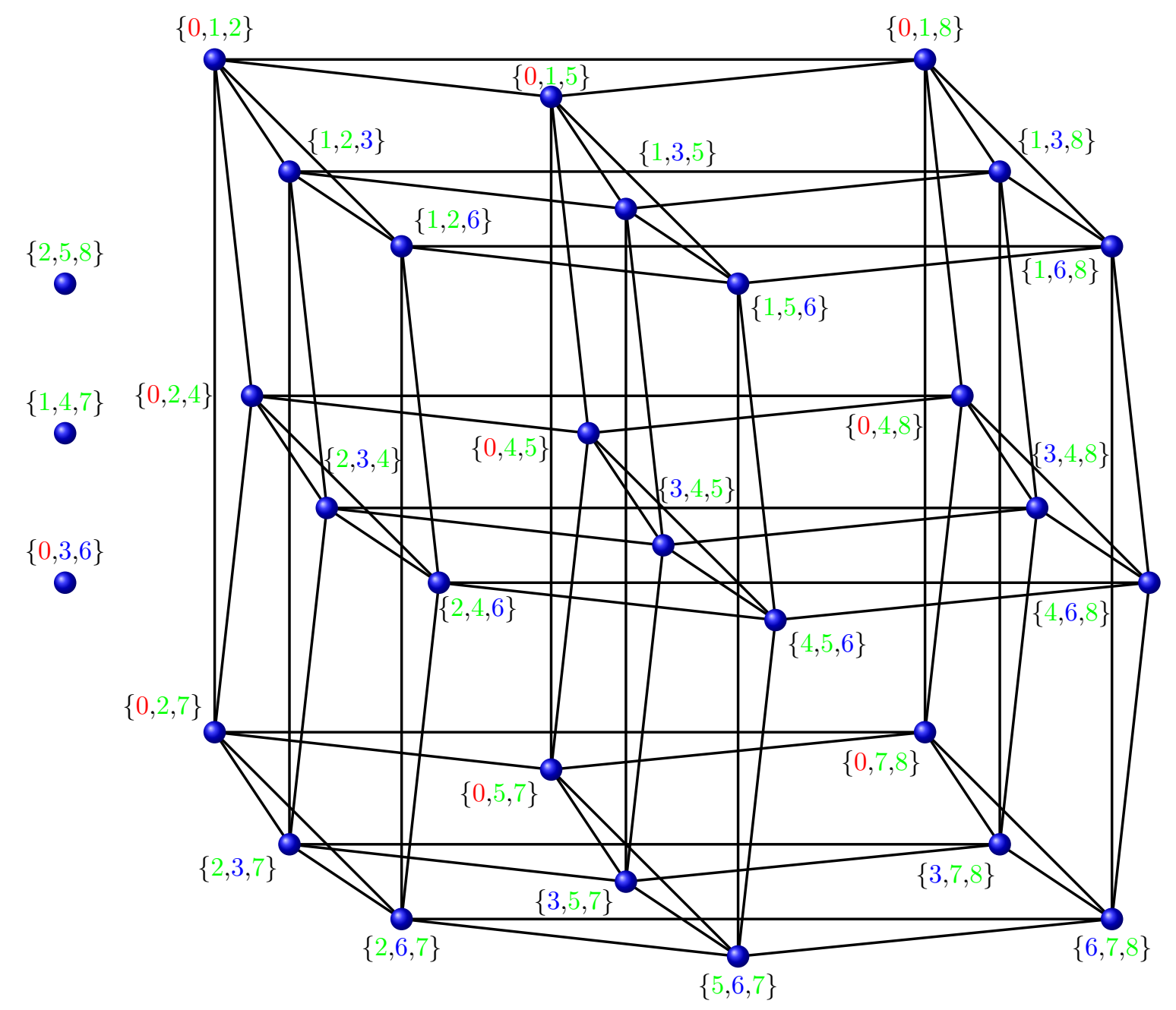

Figura 5.11: La gráfica $G_{3}\left(\vec{C}_{3}\left[\vec{C}_{3}\right]\right)$.

trayectoria

$$
P: u_{1} \rightarrow u_{2}, \rightarrow u_{3} \rightarrow \ldots \rightarrow u_{n} \rightarrow w_{1}
$$

en la subyacente de $B_{v}(T)$. Notemos en la figura 5.12, que esta trayectoria proporciona la sucesión de triángulos no ajenos en flechas

$$
\begin{aligned}
\overrightarrow{C_{3}}(1) & : \quad v \rightarrow u_{1} \rightarrow w_{1} \rightarrow v, \\
\overrightarrow{C_{3}}(2) & : \quad v \rightarrow u_{1} \rightarrow u_{2} \rightarrow v, \\
\overrightarrow{C_{3}}(3) & : \quad v \rightarrow u_{3} \rightarrow u_{2} \rightarrow v, \\
\vdots & \\
\vec{C}_{3}(n-1) & : v \rightarrow u_{n} \rightarrow u_{n-1} \rightarrow v, \\
\vec{C}_{3}(n) & : \quad v \rightarrow u_{n} \rightarrow w_{n} \rightarrow v .
\end{aligned}
$$




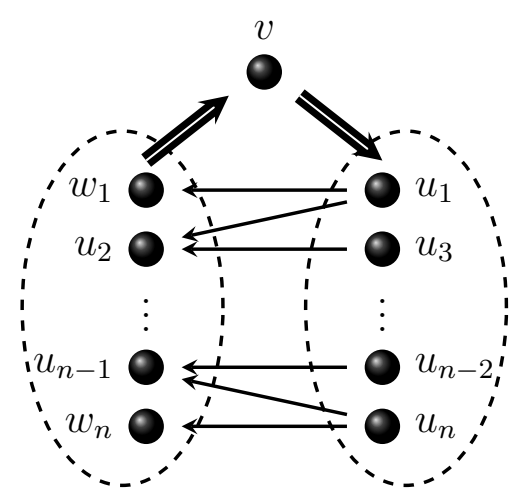

Figura 5.12: Sucesión de triángulos no ajenos en flechas.

Lo anterior señala que en $G_{3}(T)$ existe una trayectoria entre los 3-vértices $e_{\left\{v, u_{1}, w_{1}\right\}}$ y $e_{\left\{v, u_{n}, w_{n}\right\}}$. Así, tenemos que si fijamos un vértice $v \in V(T)$, entonces la subgráfica que resulta de eliminar las flechas entre de los conjuntos $N^{+}(v)$ y $N^{-}(v)$ resulta ser conexa, es decir

$$
G_{3}\left(T \backslash\left\{A\left(N^{+}(v)\right) \cup A\left(N^{-}(v)\right)\right\}\right)
$$

es conexa para toda $v \in V(T)$. Por lo tanto $G_{3}(T)$ es conexa.

De las proposiciones y teoremas anteriores, tenemos la siguiente caracterización.

Corolario 5.4. Sea $T$ un torneo regular. Entonces $T$ es isomorfo a una suma de Zykov no trivial $B[\alpha]$ si y solo si $G_{3}(T)$ no es conexa.

Demostración. La necesidad se sigue por la proposición $5.3^{13}$ y la suficiencia por el recíproco del teorema 5.8 .

Notemos que la caracterización anterior es una nueva manera de identificar un torneo regular primo. Esta caracterización se suma a las ya antes expuestas y a continuación damos el siguiente teorema, que es una recopilación de las caracterizaciones dadas anteriormente para un torneo primo. Consideramos que este es el teorema principal de este trabajo de investigación.

Teorema 5.9 (Caracterización de los torneos regulares primos). Las siguientes proposiciones son equivalentes para un torneo regular $T$.

(i) T es primo.

(ii) $T$ es tenso.

(iii) $B_{v}(T)$ es conexa (débil) para toda $v \in V(T)$.

(iv) $G_{3}(T)$ es conexa.

\footnotetext{
${ }^{13}$ página 94
} 
Demostración. Se sigue por el teorema de caracterización $5.3^{14}$ y el corolario 5.4.

La siguiente definición, a pesar de su sencillez, nos será de gran ayuda para describir un método para factorizar un torneo regular no primo, en componentes de una suma de Zykov no trivial.

Definición 5.4. Sean $D$ una digráfica y $G_{3}(D)$ su gráfica de intersección de triángulos. Sean $G_{1}, G_{2}, \ldots, G_{s}$ las componentes conexas de la gráfica $G_{3}(T)$ y definamos el subconjunto de vértices de $D$

$$
\sigma\left(G_{i}\right)=\bigcup\left\{v \in V(D): v \in \text { e para algún 3-vértice } e \in V\left(G_{3}(D)\right)\right\}
$$

para toda $i \in\{1,2, \ldots, s\}$

Es decir, $\sigma\left(G_{i}\right)$ es la unión de los vértices de $D$ que pertenecen a algún 3-vértice en $G_{3}(D)$. Si algún vértice en $V(D)$ no pertenece a $\overrightarrow{C_{3}}$ alguno, entonces dicho vértice no está en $\sigma\left(G_{3}(D)\right)$. Así mismo, si la digráfica $D$ es un torneo regular $T$, entonces $\sigma\left(G_{3}(T)\right)=V(T)$.

La siguiente proposición permite dar una propiedad entre los conjuntos de vértices antes definidos.

Proposición 5.4. Sea $T \cong B[\alpha]$ un torneo regular tal que la base $B$ y todo miembro de la familia $\alpha$ sea un torneo primo. Como $G_{3}(T)$ no es conexa, sean $G_{1}, G_{2}, \ldots, G_{s}$ las s componentes conexas de $G_{3}(T)$ y supongamos sin pérdida de generalidad que

$$
\left|\sigma\left(G_{1}\right)\right| \leq\left|\sigma\left(G_{2}\right)\right| \leq \ldots \leq\left|\sigma\left(G_{s}\right)\right|
$$

Entonces para todo par de enteros $i, j$ tales que $1 \leq i<j \leq s$, se cumple uno y solo uno de los siguientes casos:

- $\sigma\left(G_{i}\right) \cap \sigma\left(G_{j}\right)=\emptyset$ o bien

- $\sigma\left(G_{i}\right) \subset \sigma\left(G_{j}\right)$

Demostración. Recordemos que si $\alpha$ tiene al menos dos componentes no triviales $\alpha_{i}$ y $\alpha_{j}$, entonces por el lema $1.3^{15}$ son torneos regulares. Por la proposición $5.3^{16}$, tenemos que $\alpha_{i}$ y $\alpha_{j}$ inducen componentes conexas distintas en $G_{3}(T)$, es decir $G_{3}\left(\alpha_{i}\right)=G_{i}$ y $G_{3}\left(\alpha_{j}\right)=G_{j}$ son componentes conexas de $G_{3}(T)$. Es claro que $V\left(\alpha_{i}\right) \cap V\left(\alpha_{j}\right)=\emptyset$, por lo que

$$
\sigma\left(G_{3}\left(\alpha_{i}\right)\right) \cap \sigma\left(G_{3}\left(\alpha_{j}\right)\right)=\emptyset
$$

y se tiene el primer caso.

\footnotetext{
${ }^{14}$ página 82

${ }^{15}$ página 14.

${ }^{16}$ página 94.
} 
Para el segundo caso, supongamos sin pérdida de generalidad que $\alpha_{1}$ es la componente no trivial de $\alpha$ asociada a $v_{1} \in V(B)$ y que $G_{1}=G_{3}\left(\alpha_{1}\right)$. Notemos que como la base $B$ es fuertemente conexa, entonces todo vértice pertenece a un $\vec{C}_{3}$. Supongamos que $v_{1}$ está en el triángulo

$$
\overrightarrow{C_{3}}: v_{1} \rightarrow v_{i} \rightarrow v_{j} \rightarrow v_{1}
$$

en la base $B$, por lo que $e_{\left\{u_{1}, u_{i}, u_{j}\right\}} \in V\left(G_{3}(T)\right)$ para todo triángulo

$$
\overrightarrow{C_{3}}: u_{1} \rightarrow u_{i} \rightarrow u_{j} \rightarrow u_{1}
$$

con $u_{1} \in V\left(\alpha_{1}\right), u_{i} \in V\left(\alpha_{i}\right)$ y $u_{j} \in V\left(\alpha_{j}\right)$. Más aún, todo 3-vértice $e_{\left\{u_{1}, u_{i}, u_{j}\right\}}$ pertenece a la misma componente conexa.

Supongamos que $e_{\left\{u_{1}, u_{i}, u_{j}\right\}} \in V\left(G_{k}\right)$. Notemos que cada vértice de $\alpha$ está en $\sigma\left(G_{k}\right)$, por lo tanto $\sigma\left(\alpha_{1}\right) \subset \sigma\left(G_{k}\right)$.

La proposición anterior señala que si el torneo regular $T$ es una suma de Zykov no trivial $B[\alpha]$, entonces todo miembro $\alpha_{i}$ de la familia $\alpha$, induce una componente conexa en $G_{3}(B[\alpha])$. Así mismo, existe una componente conexa que contiene a cada vértice del torneo $T$.

Esta propiedad proporciona un método para descomponer un torneo no simple como una suma de Zykov no trivial. Por el teorema de caracterización de los torneos regulares primos $5.9^{17}$, tenemos que el torneo $T$ es una suma de Zykov no trivial si y solo si $G_{3}(T)$ no es conexa.

Es bien sabido que dado un torneo regular $T$, el decidir si $T$ es isomorfo o no a una suma de Zykov no trivial es extremadamente difícil, pues responder dicha pregunta, supondría realizar todas las posibles particiones del conjunto de vértices de $T$ y determinar si alguna de ellas nos proporciona la partición deseada, es un problema exponencial. Sin embargo, la proposición anterior define un método fácil para determinar las componentes de la suma de Zykov no trivial.

Observación 5.2 (Factorización de torneos regulares). Sea $T$ un torneo regular tal que $G_{3}(T)$ no es conexa. Sean $G_{1}, G_{2}, \ldots, G_{s}$ las componentes conexas, tales que

$$
\left|\sigma\left(G_{1}\right)\right| \leq\left|\sigma\left(G_{2}\right)\right| \leq \ldots \leq\left|\sigma\left(G_{s}\right)\right|
$$

Entonces $T$ puede ser factorizada como una suma de Zykov no trivial $B[\alpha]$, donde todo elemento $\alpha_{i}$ de la familia $\alpha$ se obtiene mediante el algoritmo descrito en la siguiente página.

Sea $T$ el torneo regular $\vec{C}_{3}\left[\vec{C}_{3}\right]$ y supongamos que desconocemos su factorización como una suma de Zykov. La gráfica $G_{3}(T)$ se observa en la figura 5.11, en la cual podemos identificar que tenemos cuatro componentes conexas, de las cuales se encuentran los 3-vértices singulares $G_{1}=v_{\{0,3,6\}}, G_{2}=v_{\{1,4,7\}}$ y $G_{3}=v_{\{2,5,8\}}$.

\footnotetext{
${ }^{17}$ página 98.
} 


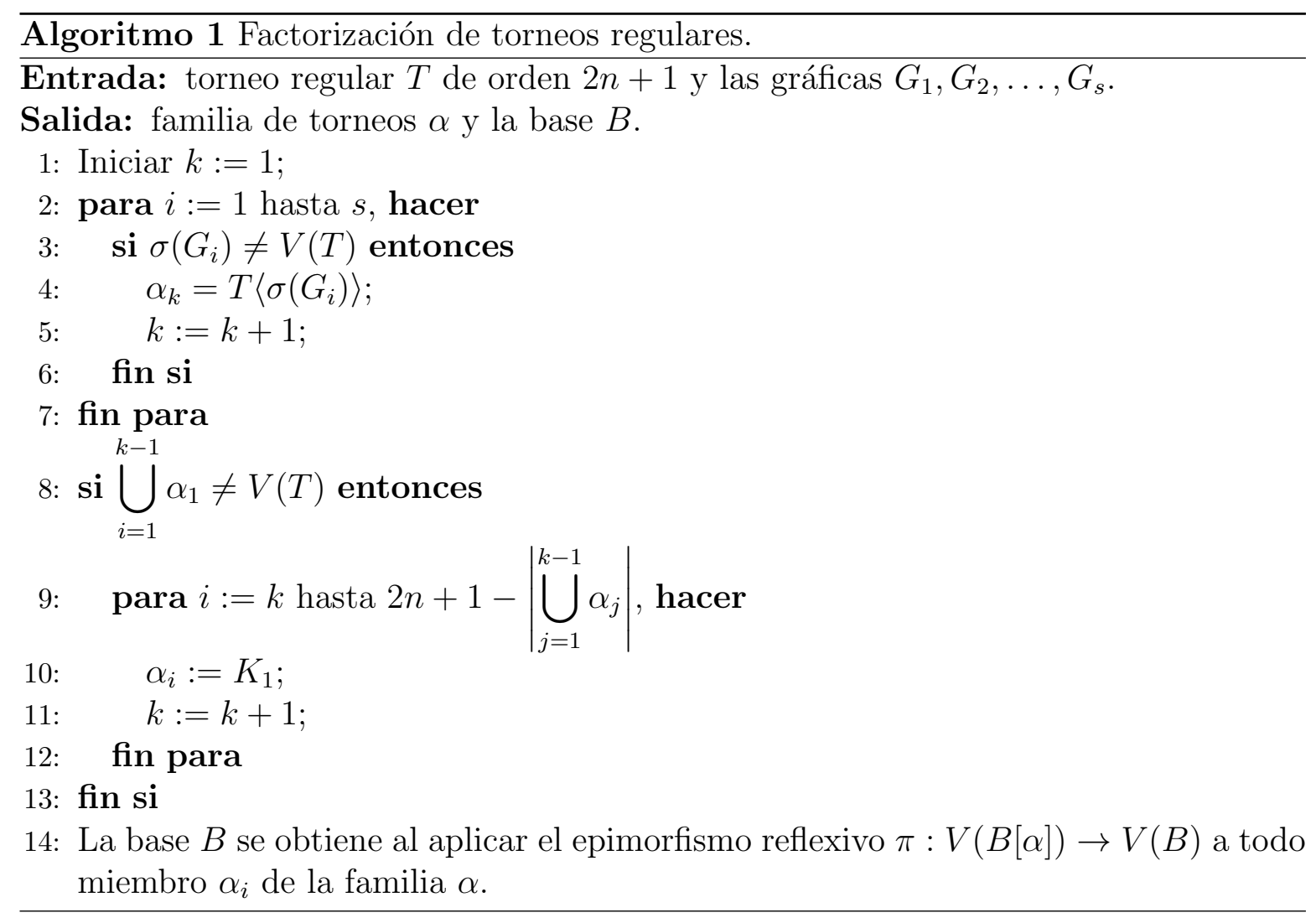

Así $\sigma\left(G_{1}\right)=\{0,3,6\}, \sigma\left(G_{2}\right)=\{1,4,7\}$ y $\sigma\left(G_{3}\right)=\{2,5,8\}$.

Esta partición de los vértices de $V(T)$ proporciona la familia $\alpha=\left\{\alpha_{1}, \alpha_{2}, \alpha_{3}\right\}$ de la suma de Zykov $B[\alpha]$, donde

$$
\begin{aligned}
& \alpha_{1}=\overrightarrow{C_{3}(1)}: 0 \rightarrow 3 \rightarrow 6 \rightarrow 0, \\
& \alpha_{2}=\vec{C}_{3}^{(2)}: 1 \rightarrow 4 \rightarrow 7 \rightarrow 1 \quad \mathrm{y} \\
& \alpha_{3}=\vec{C}_{3}^{(3)}: 2 \rightarrow 5 \rightarrow 8 \rightarrow 0 .
\end{aligned}
$$

La base se determina al aplicar el epimorfismo reflexivo $\pi: V(T) \rightarrow V(B)$, es decir

$$
B=\overrightarrow{C_{3}}: \pi\left(\alpha_{1}\right) \rightarrow \pi\left(\alpha_{2}\right) \rightarrow \pi\left(\alpha_{3}\right) \rightarrow \pi\left(\alpha_{1}\right) .
$$

Otro ejemplo es si tomamos al torneo $T \in \mathfrak{U}$ de la figura $3.4^{18}$, entonces la gráfica $G_{3}(T)$ tiene solo dos componentes conexas, de las cuales se encuentra el 3-vértice $u_{\{0,3,6\}}$. Notemos que en la suma de Zykov de este caso, solo se obtiene una componente no trivial $\alpha_{1}=\overrightarrow{C_{3}}: 0 \rightarrow 3 \rightarrow 6 \rightarrow 0$ y los restantes ocho elementos de $\alpha$ son vértices singulares.

Ahora bien, si $T \in \mathfrak{U}$ es el torneo de la figura $3.5^{19}$, entonces la gráfica $G_{3}(T)$ tiene tres componentes conexas, de las cuales, están los 3 -vértices $u_{\{0,3,6\}}$ y $u_{\{2,5,8\}}$. La suma

\footnotetext{
${ }^{18}$ página 44

${ }^{19}$ página 49
} 
de Zykov en este caso, tiene a las componentes no triviales

$$
\begin{aligned}
& \alpha_{1}=\vec{C}_{3}^{(1)}: 0 \rightarrow 3 \rightarrow 6 \rightarrow 0 \quad \mathrm{y} \\
& \alpha_{2}={\overrightarrow{C_{3}}}^{(2)}: 2 \rightarrow 5 \rightarrow 8 \rightarrow 2
\end{aligned}
$$

y los restantes cinco elementos de $\alpha$ son vértices singulares. 
En este trabajo exponemos avances significativos al problema de determinar la inconexión acíclica y la inconexión libre de $\overrightarrow{C_{3}}$ de torneos. Damos respuesta a varios problemas y conjeturas de Neumann-Lara sobre torneos y en especial, sobre torneos regulares.

Podemos destacar la construcción de las familias infinitas de torneos $\mathfrak{V}_{n}$ y $\mathfrak{U}$. La primera responde afirmativamente a dos problemas sobre la diferencia entre la inconexión acíclica y la inconexión libre de $\overrightarrow{C_{3}}$ (capítulo 2). La segunda familia exhibe contraejemplos a una conjetura sobre la tensión de los torneos regulares, que son ejemplos de torneos regulares no simples que no son tensos (capítulo 3).

Consideramos que el teorema 5.9 de la página 98, es el teorema más importante de este trabajo, el cual contiene tres caracterizaciones importantes de los torneos regulares primos.

La primera caracterización permite decidir si un torneo dado, es o no una suma de Zykov no trivial. La solución de este problema fue el objetivo primario de este trabajo (capítulo 4).

La segunda caracterización permite dar una coloración que calcula la inconexión acíclica y la inconexión libre de $\vec{C}_{3}$ a partir de analizar la conexidad de la digráfica bipartita $B_{v}(T)$. Esto es una ventaja significativa al problema de determinar la partición óptima de los vértices del torneo que realiza la inconexión acíclica y la inconexión libre de $\vec{C}_{3}$, lo que se traduce en una coloración óptima de su conjunto de vértices.

Como consecuencias de esta última caracterización, primero obtenemos un método para determinar la inconexión acíclica y la inconexión libre de $\overrightarrow{C_{3}}$ del torneo semirregular asociado. La segunda consecuencia es la obtención de una clase de torneos regulares, que son críticos en vértices con respeto a dichos parámetros (capítulo 5).

La tercera caracterización tiene como resultado un método para descomponer un torneo regular, como una suma de Zykov no trivial, lo que supone -al igual que la segunda caracterización- una ventaja significativa, porque determinar la partición óptima que define una suma de Zykov, es un problema extremadamente difícil (capítulo 5).

A continuación planteamos algunos problemas interesantes que serían una excelente continuación a este trabajo.

Problema 1. ¿Será cierto que toda componente conexa (débil) de la gráfica bipartita $B_{v}(T)$ tiene una antitrayectoria generadora para cada $v \in V(T)$ ?

Problema 2. ¿Será cierto que el único torneo regular que es $\vec{\omega}$-crítico y $\overrightarrow{\omega_{3}}$-crítico es el torneo cíclico?

Problema 3. Estudiar las propiedades de la gráfica $G_{3}(T)$, tales como hamiltonicidad de sus componentes conexas, $k$-conexidad, etc. 
Problema 4. ¿Qué se puede decir de la complejidad del algoritmo de factorización descrito en la página 101 ?

Problema 5. ¿Será cierto que si el torneo $T$ es una suma de Zykov no trivial, entonces $G_{3}(T)$ tiene una componente conexa que es isomorfa a un producto cartesiano de gráficas? Si es así, caracterice dichas gráficas.

Conjetura. Para todo torneo semirregular $S R$ se cumple que $\vec{\omega}(S R)=\overrightarrow{\omega_{3}}(S R)$. 


\section{Bibliografía}

[A67] B. Alspach, Cycles of each length in regular tournaments, Canad. Math. Bull. 10 (1967) 283-285.

[ABNL92] J. Arocha, J. Bracho and V. Neumann-Lara, On the minimum size of tight hipergraphs, J. Graph Theory 16 (1992) 319-326.

[ABNL95] J. Arocha, J. Bracho and V. Neumann-Lara, Tight and untight triangulated surfaces, J. Combin. Theory B 63 (1995) 185-199.

[BJG08] J. Bang-Jensen and G. Guting Digraphs: Theory, Algorithms and Applications, 2nd Edition, Springer-Verlag London Ltd, 2008.

[BR79] L. W. Beineke and K. B. Reid, Tournaments, in L. W. Beineke, R. J. Wilson (Eds.), Selected Topics in Graph Theory, Academic Press, New York, 1979, 169-204.

[BM76] J. A. Bondy and U. S. R. Murty, Graph Theory with Aplications, American Elservier Pub. Co., 1976.

[CDKL98] H. Cho, F. Doherty, S-R. Kim and R. Lundgren, Domination graphs of regular tournaments II, Congr. Numer. 130 (1998) 95-111.

[CKL98] H. Cho, S-R. Kim and R. Lundgren, Domination graphs of regular tournaments, Discrete Math. 252 (2002) 57-71.

[CALl13] J. L. Cosme-Álvarez and B. Llano, The acyclic and $\vec{C}_{3}$-free disconnection of tournaments, Discrete Math. 313 (2013) 2348-2353.

[FGLMR01] D. C. Fisher, D. Guichard, J. R. Lundgren, S. K. Merz and K. B. Reid, Domination graphs whith nontrivial components, Graphs Combin. 17 (2001) 227-236.

[FL99] D. C. Fisher and J. R. Lundgren, Connected domination graphs of tournaments, J. Combin. Math. Combin. Comp. 31 (1999) 169-176.

[FLMR98] D. C. Fisher, J. R. Lundgren, S. K. Merz and K. B. Reid, The domination and competition graphs of a tournament J. Graph Theory 29 (1998) 103110.

[GSNL00] H. Galeana-Sánchez and V. Neumann-Lara, A class of tight circulant tournaments, Discuss. Math. Graph Theory 20 (2000) 190-228.

[GKO91] W. D. Goddard, G. Kubicki, O. R. Oellermann and S. L. Tian. On multipartite tournaments, J. Combin. Theory Ser. B, 52(2):284-300, 1991. 
[IKH11] W. Imrich and S. Klavžar, Product Graphs. Structure and recognition, Wiley-Interscience Series in Discrete Mathematics and Optimization. Wiley-Interscience, New York, 2000.

[JM83] H. Jacob and H. Meyniel, Extension of Turan's and Brooks'theorems and a new notion of stability and coloring in digraphs, Ann. Discrete Math. 17 (1983) 365-370.

[Ll] B. Llano, Two characterizations of simple circulant tournaments, (submitted).

[LlO07] B. Llano and M. Olsen, Infinite families of tight regular tournaments Discuss. Math. Graph Theory, 27 (2007) 299-311.

[LlNL07] B. Llano and V. Neumann-Lara, Circulant tournaments of prime order are tight, Discrete Math. 308 (2007) 6056-6063.

[Mc] B. McKay, Combinatorial Data, http://cs.anu.edu.au/ ${ }^{b d m / d a t a / d i g r a p h s . h t m l ~}$

[MJW68] J. W. Moon, Topics on Tournaments, Holt, Rinehart \& Winston, New York, 1968.

[N96] M. B. Nathanson, Additive Number Theory: Inverse Problems and the Geometry of Sumsets, in: Graduate Texts in Math., vol. 165, SpringerVerlag, New York, 1996.

[NL] Comunicación personal.

[NL82] V. Neumann-Lara, The dichromatic number of a digraph, J. Combin. Theory (B) 33 (1982) 256-270.

[NL99] V. Neumann-Lara, The acyclic disconnection of a digraph, Discrete Math. 197/198 (1999) 617-632.

[NLO09] V. Neumann-Lara and M. Olsen, Tame tournaments and their dichromatic number, Austr. J. of Comb., 45 (2009), 25-35.

[NU83] V. Neumann-Lara and J. Urrutia, Vertex critical r-dichromatic tournaments, Discrete Mathematics 49 (1984) 83-87.

[R04] K. B. Reid, Tournaments, in: Jonathan Gross, Jay Yellen (eds.), Handbook of Graph Theory, CRC Press, 2004, 156-182 